\title{
Adaptive Actuator Failure Compensation Control of Robotic Systems with Uncertainties
}

\author{
A Thesis \\ Presented to
}

the faculty of the School of Engineering and Applied Science

University of Virginia

\author{
in partial fulfillment \\ of the requirements for the degree \\ Doctor of Philosophy \\ by \\ Thummaros Rugthum
}

August 2017 



\title{
APPROVAL SHEET
}

\author{
This Thesis \\ is submitted in partial fulfillment of the requirements \\ for the degree of \\ Doctor of Philosophy
}

Author Signature:

This Thesis has been read and approved by the examining committee:

Advisor: Prof. Gang Tao

Committee Member: Prof. Zongli Lin

Committee Member: Prof. Peter A. Beling

Committee Member: Prof. Harry Powell

Committee Member: Prof. Scott T. Acton

Committee Member:

Accepted for the School of Engineering and Applied Science:

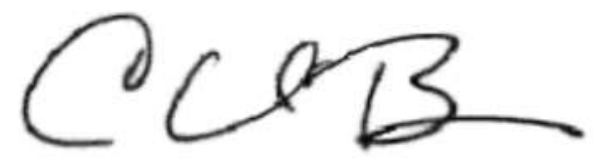

Craig H. Benson, School of Engineering and Applied Science 



\section{Abstract}

This research develops an adaptive actuator failure compensation framework of robotic systems with parameter uncertainties in addition to actuator failures. The research shows a complete design methodology for developing an actuator failure compensation scheme for robotic systems. The adaptive control design uses an integration of multiple individual failure compensators, which utilizes existing control techniques such as the backstepping control design. With the direct adaptation based on the Lyapunov technique to handle uncertainties in the system, the adaptive actuator failure compensation framework guarantees desired closed-loop stability and asymptotic output tracking, despite actuator failures whose patterns, times and values are all unknown. Simulation results are presented to verify the desired adaptive actuator failure compensation control performances for multiple types of robotic systems from the theoretical cooperative manipulator benchmark system to a more realistic application such as the landing of a helicopter with robotic legs. 


\section{Acknowledgement}

The writing of this thesis has been one of the most significant academic challenges of my life. Without the help and guidance of the following people, I would not be able to complete my study. First of all, I would like to express my deepest gratitude to my advisor and mentor, Dr. Gang Tao, for his support, caring, encouragement, patience, and providing me with endless opportunities in pursuing my research as well as acquiring teaching experience, which is crucial to my career. Secondly, I would like to thank my thesis committee who guided me through all these years.

Special thanks to my family. My family always loves and supports me unconditionally. I will not be able to get to this point in my life without them. Also, I would

like to thank all of my friends who supported me in this writing, and encouraged me to strive towards my goal. 


\section{Contents}

1 Introduction 1

1.1 Literature Review . . . . . . . . . . . . . . . . . . . . . . 2

1.2 State of the Art . . . . . . . . . . . . . . . . 4

1.2.1 Fault Tolerant Control for Robotic Systems . . . . . . . . . . 5

1.2.2 Adaptive Actuator Failure Compensation Techniques . . . . . 8

1.3 Research Question and Basic Approach . . . . . . . . . . . . . 10

1.4 Thesis Outline . . . . . . . . . . . . . . . . 12

2 Technical Background $\quad 15$

2.1 Robot Manipulators . . . . . . . . . . . . . . . . . 15

2.2 Euler-Lagrange Equation . . . . . . . . . . . . . . . . . . . . . . 17

2.2.1 Example: Single-link Robotic Manipulator System . . . . . . . 20

2.2.2 Example: Two-link Planar Elbow Robotic Manipulator . . . . 21

2.3 Cooperative and Parallel Manipulators . . . . . . . . . . . . 24

2.4 Lyapunov Stability Theory . . . . . . . . . . . . . . . 25

2.5 Control of Robotic Systems . . . . . . . . . . . . . . . 27

2.5.1 Slotine and Li's Control Design . . . . . . . . . . . . . 27

2.5.2 Backstepping Nonlinear Control Design . . . . . . . . . . . . . 29

3 Problem Formulation 33

3.1 Actuator Failures in Robotic Systems . . . . . . . . . . . . . . . . 34

3.1.1 Actuator Failure Uncertainties . . . . . . . . . . . . . 34 
3.1.2 Actuator Failure Model . . . . . . . . . . . . . 35

3.1.3 Actuator Failure Pattern . . . . . . . . . . . . . . 36

3.2 Basic Problem Statements . . . . . . . . . . . . . . . . 37

3.2.1 Initial Stage of the Development . . . . . . . . . . . . 37

3.2.2 Final Stage of the Development . . . . . . . . . . . 41

4 Design for A Cooperative Manipulator System 49

4.1 Problem Formulation . . . . . . . . . . . . . . . . . . 50

4.1.1 Cooperative Manipulator Systems . . . . . . . . . . . . 50

4.1.2 Actuator Failure Model . . . . . . . . . . . . . . 51

4.1 .3 Control Objective . . . . . . . . . . . . 52

4.1.4 A Benchmark Cooperative Manipulator System . . . . . . . 54

4.2 The Benchmark System Model . . . . . . . . . . . . . . . 55

4.3 Nominal Control Design . . . . . . . . . . . . . . . . . 64

4.3.1 Backstepping Control Design ... . . . . . . . . . 64

4.3.2 Nominal Actuator Failure Controller Structure . . . . . . . . . 66

4.4 Adaptive Actuator Failure Compensation . . . . . . . . . . . . . 71

4.4.1 Adaptive Controller Structure . . . . . . . . . . . 71

4.4 .2 Error System . . . . . . . . . . . . . . . . 74

4.4 .3 Adaptive Laws . . . . . . . . . . . . . . 76

4.4.4 Performance Analysis . . . . . . . . . . . . . . . . 80

4.5 Initial Control Framework . . . . . . . . . . . . . . . . 83

4.5.1 General Dynamic Model of Robotic System . . . . . . . . . . 84

4.5.2 Actuator Failure Compensation Scheme . . . . . . . . . 86

4.6 Simulation Study . . . . . . . . . . . . . . . . . . . . . . 89

4.6.1 System Model and Simulation Conditions . . . . . . . . . 89

4.6.2 Simulation Results ................. . 90 
4.7 Conclusion . . . . . . . . . . . . . . . . . . 95

5 Design for A Hexapod System $\quad 97$

5.1 System Modeling of the Hexapod System . . . . . . . . . . . . . . . . 98

5.1.1 Dynamic Model of the Hexapod . . . . . . . . . . . . . . . . 98

5.1.2 Dynamic Model in State Space Form . . . . . . . . . . . . . . 101

5.2 Problem Formulation . . . . . . . . . . . . . . . . . . 102

5.2.1 Control Objective . . . . . . . . . . . . . . 102

5.2.2 Actuator Failure Model . . . . . . . . . . . . . . 103

5.2.3 Concurrrent Actuator Failures . . . . . . . . . . . . . . . . 104

5.3 Nominal control design . . . . . . . . . . . . . . . . . 104

5.4 Adaptive Failure Compensation Design . . . . . . . . . . . . . . . . . 110

5.4.1 Adaptive Controller Structure . . . . . . . . . . . . . . . . . 110

5.4 .2 Adaptive Laws . . . . . . . . . . . . . . . . . . . . . . . . 111

5.5 Simulations Study . . . . . . . . . . . . . . . . 117

5.5.1 System Model and Simulation Conditions . . . . . . . . . . 117

5.5.2 Simulation Results . . . . . . . . . . . . . . . . 118

5.6 Conclusions .......................... 120

6 Design for A Cooperative System with Parameter Uncertainties 123

6.1 Problem Formulation . . . . . . . . . . . . . . . . 126

6.1.1 Dynamic Model of Cooperative Manipulator Systems . . . . . 126

6.1.2 Actuator Failure Model . . . . . . . . . . . . . . . . 127

6.1.3 Basic Assumption . . . . . . . . . . . . . . . . . . . . . . 128

6.1.4 Control Objective . . . . . . . . . . . . . . . . 129

6.2 Nominal control design . . . . . . . . . . . . . . . . . 130

6.2.1 Based Line Control Design . . . . . . . . . . . . . . . . . . 131 
6.2.2 Actuator Failure Compensation . . . . . . . . . . . . 133

6.3 Adaptive Failure Compensation Design . . . . . . . . . . . . . . . . 137

6.3.1 Adaptive Controller Structure . . . . . . . . . . . . . 138

6.3 .2 Error System . . . . . . . . . . . . . . . . . 140

6.3 .3 Adaptive Laws . . . . . . . . . . . . . . . . . . . 141

6.3.4 Performance Analysis . . . . . . . . . . . . . . . . . 144

6.3.5 Concurrent Actuator Failures . . . . . . . . . . . . . . 147

6.4 Simulations Study . . . . . . . . . . . . . . . . . . . 152

6.4.1 The Benchmark System Model . . . . . . . . . . . . . . 152

6.4.2 Benchmark System in General Form . . . . . . . . . . . . . 153

6.4.3 Simulation Conditions . . . . . . . . . . . . . 154

6.4.4 Simulation Results . . . . . . . . . . . . . 156

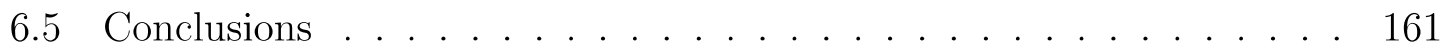

7 Design for A Parallel Manipulator with Parameter Uncertainties 163

7.1 Problem Formulation . . . . . . . . . . . . . . . . . . . 164

7.1.1 Dynamic Model of the Parallel Robots . . . . . . . . . . . . 164

7.1.2 Actuator Redundancy ................. 169

7.1.3 Control Objective . . . . . . . . . . . . . 169

7.2 Nominal control design . . . . . . . . . . . . . . . . . . . . 170

7.2.1 Based Line Control Approach . . . . . . . . . . . . . . . . . 170

7.2.2 Actuator Failure Compensation . . . . . . . . . . . . 172

7.3 Adaptive Failure Compensation Design . . . . . . . . . . . . . 175

7.3.1 Adaptive Controller Structure . . . . . . . . . . . . . 176

7.3 .2 Error System . . . . . . . . . . . . . . . . . . . 177

7.3 .3 Adaptive Laws . . . . . . . . . . . . . . . . . 178

7.3.4 Performance Analysis . . . . . . . . . . . . . . . . . 179 
7.4 Simulations Study . . . . . . . . . . . . . . . . 181

7.4.1 2-DOF redundantly actuated parallel manipulator model . . . 181

7.4.2 Simulation Conditions ................ 183

7.4.3 Simulation Results . . . . . . . . . . . . . . 183

7.5 Conclusions ........................ 186

8 Design for Landing of A Helicopter with Robotic Legs 189

8.1 Problem Formulation . . . . . . . . . . . . . . . . . . 192

8.1.1 Dynamic Model of the System . . . . . . . . . . . . . . 192

8.1.2 Actuator Redundancy ................. 197

8.1.3 Actuator Failure Model . . . . . . . . . . . . . . . . 198

8.1.4 Control Objective . . . . . . . . . . . . . 199

8.2 Nominal control design . . . . . . . . . . . . . . . . 200

8.2.1 Ideal Control System . . . . . . . . . . . . . . . 201

8.2.2 Actuator Failure Compensation . . . . . . . . . . . . . 203

8.3 Adaptive Failure Compensation Design . . . . . . . . . . . . . 205

8.3.1 Adaptive Controller Structure . . . . . . . . . . . . 206

8.3.2 Adaptive Laws . . . . . . . . . . . . . . . . . . 209

8.3.3 Performance Analysis . . . . . . . . . . . . . . . . . . 210

8.4 Simulation Study . . . . . . . . . . . . . . . . . . 211

8.4.1 Simulation Conditions . . . . . . . . . . . . 212

8.4.2 Simulation Results . . . . . . . . . . . . . . 213

8.5 Conclusions .......................... 216

9 Conclusion and Future Works $\quad 217$

9.1 Future Works . . . . . . . . . . . . . . . . 217 
Bibliography 


\section{List of Figures}

1.1 Block diagram for nominal design . . . . . . . . . . . . . . 11

1.2 Block diagram for adaptive failure compensation design . . . . . . . . 11

2.1 Two-link planar elbow robotic manipulator. . . . . . . . . . . . 22

3.1 The model of Hexapod and the manipulator structure. . . . . . . . . 40

3.2 The 2-DOF redundantly actuated parallel manipulator. . . . . . . . . 44

3.3 Landing of a helicopter with robotic legs on uneven terrains with redundantly actuated parallel manipulators. . . . . . . . . . . . . . . 47

4.1 The benchmark cooperative robotic system. . . . . . . . . . 55

4.2 System responses for $y_{m}(t)=[2+\sin (0.1 t), 0.5]^{T}$ with constant failure. 91

4.3 Actuator outputs subject to constant actuator failure. . . . . . . . . . 92

4.4 Adaptive indicator functions $\chi_{11}, \chi_{12}, \chi_{13} \ldots \ldots . \ldots 92$

4.5 Adaptive indicator functions $\chi_{21}, \chi_{22}, \chi_{31}, \chi_{32} \ldots \ldots . \ldots 93$

4.6 System responses for $y_{m}(t)=[2+\sin (0.1 t), 0.2+0.1 \sin (0.1 t)]^{T}$ with sinusoidal actuator failure. . . . . . . . . . . . . . . . 93

4.7 Actuator outputs subject to sinosoidal actuator failure. . . . . . . . . 94

4.8 Adaptive indicator functions $\chi_{11}, \chi_{12}, \chi_{13} \ldots \ldots . \ldots . \ldots 94$

4.9 Adaptive indicator functions $\chi_{21}, \chi_{22}, \chi_{31}, \chi_{32} \ldots \ldots \ldots$

5.1 The model of Hexapod and the manipulator structure. . . . . . . . . 99

5.2 System output $\theta(t)$ vs. $y_{m}(t)$ for one actuator failure case. . . . . . 119 
5.3 System output $\theta(t)$ vs. $y_{m}(t)$ for two actuator failure case. . . . . . . 119

5.4 System output $\theta(t)$ vs. $y_{m}(t)$ for three actuator failure case. . . . . . 120

6.1 The benchmark cooperative manipulator system. . . . . . . . . . . . 152

6.2 System output $x_{o}(t)$ vs. $x_{d}(t)$ for the lock in-place failure. . . . . . . 156

6.3 Estimated parameters $\hat{\theta}_{1}$ for the lock in-place failure. . . . . . . . . . 157

6.4 Estimated parameters $\hat{\theta}_{1}$ for the square wave actuator failure. . . . . 158

6.5 Estimated actuator failure signal $\rho_{1(1)}, \rho_{1(2)}, \rho_{2(1)}$ and $\rho_{2(2)} \ldots \ldots$. . 158

6.6 System output $x_{o}(t)$ vs. $x_{d}(t)$ for the square wave actuator failure. . . 159

6.7 Estimated actuator failure signal $\rho_{1(1)}, \rho_{1(2)}, \rho_{2(1)}$ and $\rho_{2(2)} . \ldots . . .160$

7.1 The 2-DOF redundantly actuated parallel manipulator. . . . . . . . . 165

7.2 System Block Diagram. . . . . . . . . . . . . . . . . . 176

7.3 System output $q_{o}(t)$ for a reference $q_{d}=\left[\begin{array}{ll}2.2 & 2.9\end{array}\right]^{T} \ldots \ldots \ldots$. . . . 184

7.4 Angle of the actuated joint $q_{a 1}, q_{a 2}, q_{a 3} \ldots \ldots \ldots \ldots$

7.5 Angle of the flexible joint $q_{b 1}, q_{b 2}, q_{b 3} \ldots \ldots \ldots \ldots$

7.6 Estimated value of the failure torque $\bar{\tau}_{1} \ldots \ldots$. . . . . . . . . 186

8.1 Landing of a helicopter with robotic legs on uneven terrains with redundantly actuated parallel manipulators. . . . . . . . . . . . . 193

8.2 Block diagram for nominal control design . . . . . . . . . . . . . 201

8.3 Adaptive system Block Diagram. . . . . . . . . . . . . . 206

8.4 The output of the system $q_{o}$ for $q_{d}=[1,0,0] \ldots \ldots \ldots$. . . . . 213

8.5 The estimate of the actuator failure pattern $\chi_{1}$ (no actuator failure case).214

8.6 The estimate of the actuator failure pattern $\chi_{2}\left(\tau_{1}\right.$ failure case). . . . 214

8.7 The estimate of the actuator failure pattern $\chi_{2}\left(\tau_{1}, \tau_{3}\right.$ and $\tau_{5}$ failures). 215 


\section{Chapter 1}

\section{Introduction}

With the exponential increase of the world population, the problem of limited resources becomes much more severe as time goes on. In order to help solve this problem to increase the supply to match the increasing demand, more advanced technologies are needed. Robotic is one of the most important technology that could help reduce the workload of human and increase productivity in various industries. Additionally, robotic system could be used to perform dangerous tasks such as search and rescue operations, space explorations, and underwater missions. Thus, the development of the robotic technology is one of the most important topics that needed our attention. One of the most important problems that prevents the advancement in robotic field is the safety issue. Since in most cases the robotic system needs to operate autonomously, any fault in the robotic system could disrupt or compromise the entire operation. In some case, a failure in the robotic system could also result in the loss of human life, who operate or work in the proximity of the system. To solve the aforementioned problem, the development of an adaptive actuator failure compensation scheme that could enable the robotic system to operate even when failure occurs is needed. Our research has been focused on the development of a new general theoretical framework for adaptive actuator failure compensation of robotic systems. The control framework is developed for designing a controller for robotic systems with possible actuator failures in addition to parameter uncertainties. The 
control framework guarantees the desired stability and tracking performance of the robotic systems.

\section{$1.1 \quad$ Literature Review}

Robotic systems play a critical role in various industries as well as in many critical projects such as search and rescue operations, space explorations, and underwater missions. In those applications, robotic systems have to operate in extreme environments $[1,2]$, which increases the chance of actuator failures in the system. Since the robotic systems are used for critical tasks [3, 4], failures may cause severe consequences. In order to increase the reliability of the system, we need to develop a controller that can function when failures occur. There are many studies that develop a controller to improve the robustness of a robotic system $[5,6,7,8,9]$. However, most of them rely on actuator failure knowledge, which often difficult to obtain in real time. Thus, a complete control design framework for adaptive actuator failure compensation schemes, which can operate without the knowledge of actuator failure, is a significant research topic. In this research, we look into two types of robotic system, which are the cooperative manipulator robotic system and the parallel structure robotic system. One of the most important characteristic of robot manipulators, which make the manipulator difficult to control, is that the dynamic of the manipulators is highly nonlinear. Although it is possible to design a controller to achieve a good performance if we known a precise model of the system [15], the controller is not capable of handling any changes and uncertainties in the system. In general a robot manipulator often interacts with environments such as grabbing or carrying different objects with unknown dimensions, orientations or gripping points. Hence, although it is possible to obtain the parameters in the system with sufficient accuracy, it is 
not practical to use parameter identification to obtain system parameters for every circumstance.

To deal with the uncertainty, the problem of designing adaptive control laws for rigid-robot manipulators that ensure asymptotic trajectory tracking has interested researchers for many years and many adaptive control algorithms have been proposed $[10,11,12,13]$. The basic idea of adaptive control is to change the values of gains or parameters in the control law according to some online algorithm corresponding to the changes and uncertainties in the system. The adaptive controller can learn an appropriate set of controller parameters during the course of its operation. A key point of the adaptive control algorithm is to make the tracking error converges regardless of whether the trajectory is persistently exciting or not [18]. That is, we do not need parameter convergence for task convergence. Moreover, the overall stability and convergence of the combined on-line control process can also be systematically guaranteed. This control approach is especially useful for robotic manipulators, which usually perform repetitive tasks. The tracking performance of the adaptive controller can improve with time through the adaptation for each successive operation [26]. Over the past two decades, the concept of using cooperative manipulators has attracted the attention of many researchers. However, most literature focuses on the studies of kinematics and dynamics of the system [22, 23]. Researches on control strategies are relatively few especially regarding to actuator failure compensation problem. In the literature, there are two types of basic control strategies for cooperative manipulators: kinematic control strategies and dynamic control strategies. In the kinematic control strategies, the nonlinear dynamics are not considered to avoid the complex computation, thus, the controller can be designed easily. However, these types of controllers can not always produce satisfactory performance, and there is no guarantee of stability especially at higher speed [24]. On the other hand, the dynamic control 
strategies, which are considered in this study use a full dynamic model of the system. In this way, the nonlinear dynamics of the landing mechanism can be compensated and higher performance can be achieved [25, 26, 27]. Finally, this control approach allows for actuator failure compensation of the landing mechanism, which is the main focus of this study.

Actuator failure compensation is a well-known problem in automatic control. There are studies that focus on actuator failure compensation, such as the neural network control, sliding-mode control, and an adaptive actuator failure compensation $[28,29]$. However, most studies only focus on actuator degradation which only consider small changes in the actuator outputs, or rely on the detection and isolation scheme of actuator failures in the system which is relatively slow and its used is limited [30, 31, 32, 33]. In order to efficiently compensate actuator failures in the helicopter landing process, an actuator failure compensation control scheme that is reliable and capable of immediate failure adaptation is needed [34, 35].

\subsection{State of the Art}

Actuator failure compensation is one of the most important problems in the robotic field. There are many studies that are trying to solve this problem. Before we propose our adaptive actuator failure compensation scheme for robotic system, we first explain the existing control technique that relates to our study. In this section, we explore some of the most recent studies regarding the fault tolerant control technique for robotic systems as well as the state-of-the-art of the adaptive actuator failure compensation outside the robotic field. The literature review of the state-of-the-art will help us understand the advantages and disadvantages of our proposed control scheme as well as explain the contribution of our study. 


\subsubsection{Fault Tolerant Control for Robotic Systems}

In this subsection, we explore the existing technique relates to fault tolerant control and actuator failure compensation for robotic system. From the recent literature, the existing techniques use for actuator failure compensation of a robotic system is the fault detection, isolation, and recovery (FDIR), which is classified as a subfield of control engineering. The existing algorithm focus on monitoring a system, identifying when an actuator failure has occurred, and obtaining the characteristics of the failures if possible. There are two major approaches which are: 1) a direct pattern recognition of sensor readings that indicate a fault and 2) an analysis of the discrepancy between the sensor readings and expected values, based on from some system model. In order to detect actuator failures in the system, we check if the discrepancy of the signal goes above a certain threshold. After the problem is detected, a fault isolation and reconfiguration is used based on the type and the location of the failure, this technique is called fault detection and isolation (FDI) techniques. The development in the fault detection, isolation, and recovery of the robotic system is still in a very early state as we can see from the recent studies. Over the past few years (2014-2016), there are several papers that try to address multiple issues in the fault detection and isolation (FDI) problem for robotic systems as follows:

In [36], the paper addresses the fault detection and isolation (FDI) problem for robotic assembly of electrical connectors. The paper considers different switched linear models with known switching sequences, bounded parameters, and external disturbances. Given a current input/output data, the feasible parameter set of faultfree switched linear model is obtained by sequentially calculating an optimal ellipsoid. If the pair of data is not consistent with any possible model, a fault is then detected. The isolation of fault is realized by checking the consistency between the data sequence 
and each possible actuator failure model one by one. In this case if a fault is detected, the robot then stops to prevent potential damage.

In [37], the paper presents a conceptual control approach that aims to integrate the fault tolerance technique into the design of a robot real-time control architecture. In this case, the fault detection is designed based on dedicated software components scanning faulty behaviors. Diagnosis is based on the residual principle and signature analysis to identify faulty software or hardware components and faulty behaviors. Finally, the overview of the recovery mechanism is discussed in different contexts and operational functions of the robotic systems.

In [38], this paper is concerned with the distributed fault estimation for a class of nonlinear networked systems, where the T-S fuzzy model is utilized to approximate the nonlinear plant and the whole fault estimation task is operated by a wireless sensor network. The controller is designed based on the Lyapunov stability theory and the robust control approach, a sufficient condition is obtained, such that the estimation error system is asymptotically stable with a prescribed $H_{\infty}$ performance level.

In [39], this paper presents an adaptive-based fault detection and isolation scheme for a general class of robot manipulators, where all parameters in the system are known. The proposed algorithm consists of a nonlinear adaptive fault detection estimator and a bank of fault isolation estimators to determine the types of faults, while the fault parameter function may be time-varying. The method is applied to a two-link robot manipulator and the simulation results are presented and discussed.

In [40], computational intelligence techniques are being investigated as an extension of the traditional fault diagnosis methods. This paper presents a scheme for fault detection and isolation (FDI) via artificial neural networks and fuzzy logic. It deals with sensors and actuator fault of a three links Scara robot. A fault is detected and 
isolated whenever the residual exceeds its corresponding threshold. The paper only considers a partial actuator failure, which we can directly adjust the control input to compensate for the failures.

In the patent [41], a fault tolerant controller is developed for robotic systems. The robotic arm supervisor may detect faults affecting an arm and also perform fault reaction activities. The system supervisor handles the fault as either a system or local fault depending upon its class. For system faults, a fault notification is sent to the arm processors of non-failed arms so that the non-failed arms are placed in the safe state. For local faults, a degraded operation option is provided and if the fault is classified as recoverable, a recovery option is provided.

As we can see from the recent literature related to actuator failure in robotic system, many studies are still in a very early state and there are many problems that still remain unsolved. The FDI approach may be slow because the error from the actuator failures need to reach certain thresholds (to prevent fault positive) before the isolate scheme can be deployed. Moreover, the isolation mechanism also prevents reactivation of the actuator even when the failures have been resolved. Another important issue with the fault detection, isolation, and recovery technique is the fact that in many cases, it may be impossible to specify the characteristic of actuator failures. This problem can be seen from the system identification technique in adaptive control. In order to guarantee the exact value of parameters, the system needs to satisfy the persistent excitation condition, which cannot be guaranteed in the operation of robotic systems.

In order to improve the performance of robotic systems with possible actuator failure, in this study, we proposed adaptive actuator failure compensation scheme for robotic system to solve the actuator failure problem, where actuator failure values, failure times and failure patterns are unknown. 


\subsubsection{Adaptive Actuator Failure Compensation Techniques}

Adaptive control is widely used for actuator failure compensation for its capability of accommodating system parameters and uncertainties. Adaptive control of system with actuator failures is aimed at compensating for uncertain failures with adaptive tuning of controller parameters based on the system error signal in order to achieve desired control performance. In this subsection, we explore recent studies of adaptive actuator failure compensation across multiple applications. The literature review would help us understand the existing algorithm related to adaptive actuator failure compensation, which would be helpful for developing the adaptive control scheme for robotic systems.

In [43], an adaptive output feedback control scheme is proposed for a class of nonlinear systems with possible actuator failures. By developing a high-gain observer with one dynamic gain, the closed-loop stability and arbitrarily small tracking error can be guaranteed. Furthermore, by estimating the upper bound of the unknown time-varying parameters caused by the actuator failures, together with an initialization technique, it is proved that the $L_{1}$ tracking performance is achievable, which is fundamentally different from the commonly accepted concept that with actuator failures, it is impossible to improve the $L_{\infty}$ tracking performance by using initialization technique.

In [44], this paper investigates the problem of adaptive fault-tolerant control for a class of linear systems with time-varying actuator faults. An active fault compensation control law was designed in two steps. Firstly, the time-varying fault parameters were estimated based on a novel adaptive observer. Then, based on online estimations of the fault parameters, an adaptive fault-tolerant controller was constructed to compensate for the loss of actuator effectiveness and to eliminate the effect of 
fault estimation error. The asymptotic stability and an adaptive $H_{1}$ performance of a closed-loop system can be guaranteed, even in the case of actuator faults and disturbances.

In [45], An optimal adaptive compensation control scheme is proposed for a class of multi-input multi-output affine nonlinear systems with actuator failures. The paper considers stuck actuators and partial effectiveness failures, an adaptive dynamic programming method is adopted by using neural networks to approximate the cost function. The proposed optimal adaptive compensation law can guarantee that the closed-loop system with actuator failures is stable and that the given reference signals are effectively tracked.

In [46], this paper solves the adaptive output rejection problem of unmatched input disturbance, and the adaptive compensation problem of uncertain failures whose pattern, values and time are all unknown, for nonminimum phase systems, two related open problems to which existing adaptive control designs are not applicable.

In [47], This paper develops a multivariable multiple-model adaptive control scheme for adaptive state feedback state tracking control of systems whose plant-model matching conditions are uncertain and parameters are unknown. The paper uses multiple reference model systems to generate multiple parameter estimations and feedback control signals from which a most suitable control input is selected. The proposed control design could guarantee both stability and asymptotic tracking performance.

In [48], This paper develops a new adaptive multiple-model control scheme for a class of nonlinear multiple-input multiple-output dynamic systems with known system parameters to compensate uncertain actuator failures using an error transformation. Such an adaptive control scheme ensures desired system stability and asymptotic tracking properties, despite the presence of failure uncertainties. 
From the literature review of the recent works in adaptive actuator failure compensation techniques, we can see that the adaptive control could offer a better performance in solving the actuator failure problem in robotic system. However, the existing algorithm is either based on a linear system, or require that all parameters in the system are known.

In this thesis, we develop an adaptive actuator failure compensation scheme for robotic system with actuator failures, where actuator failure values, failure times and failure patterns are unknown. In our study, we consider the nonlinear dynamic model of the robotic systems (dynamic control approach), where some parameters in the system may be unknown. The adaptive actuator failure compensation scheme can guarantee desirable closed-loop stability and asymptotic tracking of the robotic system.

\subsection{Research Question and Basic Approach}

The main objective of this study is to develop an adaptive actuator failure compensation scheme to solve actuator failure problem, where actuator failure values, failure times and failure patterns are unknown. The proposed algorithm also considers standard problems in control and robotic field such as the system constraint and parameter uncertainty, in addition to actuator failure problem.

\section{Basic approach}

In this research, the development of each control design is divided into four steps as follows. In step 1, we begin our research by formulating the problem with the discussion of control objective and the dynamic model of a robotic system. In step 2, we design a nominal controller for the system in a nominal situation in which the 
knowledge of actuator failure is known.

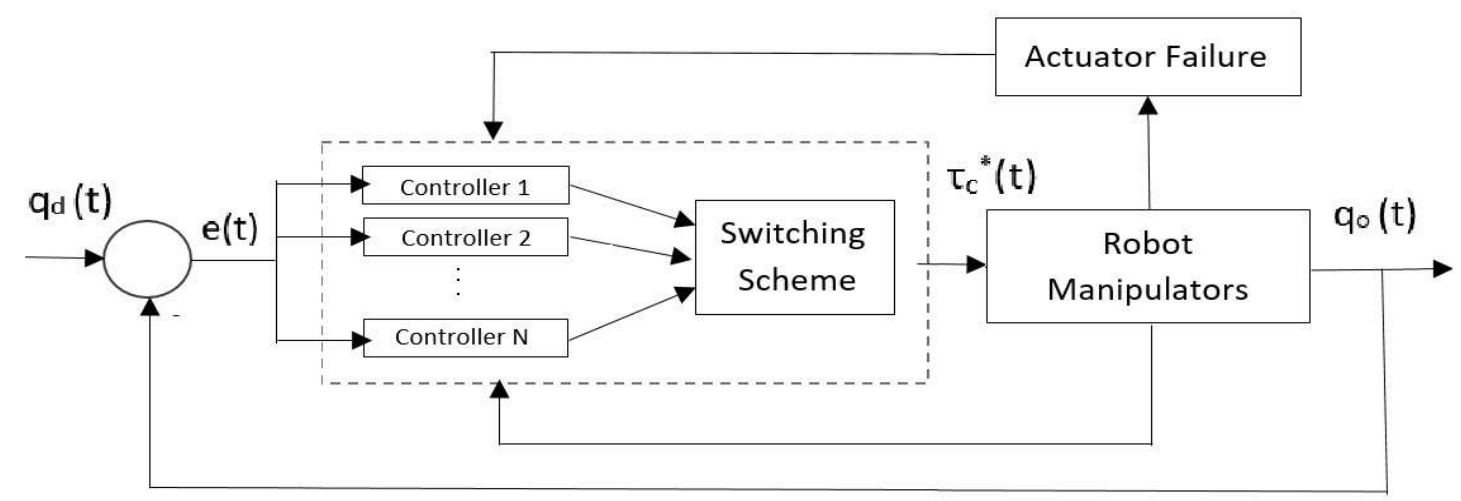

Figure 1.1: Block diagram for nominal design

From the block diagram, each nominal controller in the controller bank is developed based on conventional approach such as the backstepping control or the Slotine and Li's control design with known actuator failure values and the switching scheme is developed from the known actuator failure pattern. However, It is generally very difficult to obtain the knowledge of actuator failure in robotic systems. In step 3, the adaptive control scheme is developed based on the nominal controller structure and parameterization for failure compensation.

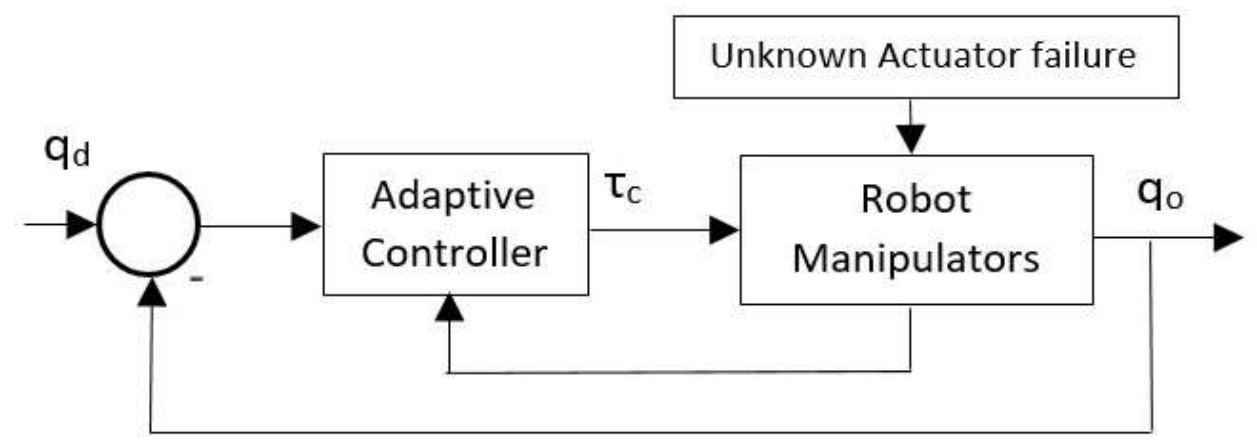

Figure 1.2: Block diagram for adaptive failure compensation design

In the adaptive failure compensation design, an adaptive integration of multiple individual failure compensators is used to combine the controllers. Since the actuator failure pattern is unknown, the switching algorithm is integrated into each adaptive 
controller. The estimators of unknown parameters and actuator failure pattern is constructed and updated using adaptive control technique using the Lyapunov theory. In step 4, we study the simulation of the designed controller with associated benchmark system to confirm its effectiveness.

\subsection{Thesis Outline}

The thesis is organized as follows:

- In Chapter 2, we introduce the fundamental background in robotic and adaptive control that is needed for the development of the proposed algorithm.

- In Chapter 3, we formulate the overall problem as well as the objective of the research.

- In Chapter 4 , we begin our study by developing an adaptive actuator failure compensation scheme based on a benchmark cooperative robotic manipulator system with known system parameter.

- In Chapter 5, we expand our knowledge of the adaptive actuator failure compensation scheme onto a generalized model of cooperative robotic systems by developing a design procedure, which can be used with various cooperative manipulator robotic systems.

- In Chapter 6, we design a controller for a robotic system with parameter uncertainty, which can better reflect a more practical type of robotic system.

- In Chapter 7, we develop an adaptive controller for a robotic system with parallel manipulator structure. In this case the problem of closed loop dynamic 
constraint is considered in addition to actuator failure and parameter uncertainty.

- In Chapter 8 , the complete framework of the adaptive actuator failure compensation control for robotic system is developed and applied to the landing of a helicopter with robotic legs.

- In Chapter 9, we summarize the result of the study and discuss some possible future works related to this research topic. 


\section{Chapter 2}

\section{Technical Background}

In this chapter, we introduce some technical backgrounds in mathematics, robotic and adaptive control that is needed for the development of the adaptive actuator failure compensation framework. Some related mathematical background and preliminaries in adaptive control will be presented for the development of the control framework in the later chapters. In Section 2.1, the basic concept and definition related to robotic system is introduced.. In Section 2.2, the Euler Lagrange's equation is introduced and examples of the derivation of the dynamic equations for robotic systems are given. Then the dynamic model of cooperative and parallel manipulator are reviewed in Section 2.3. In Section 2.4, we introduce the Lyapunov stability theory, which is the fundamental concept used in our control designs. In Section 2.5 introduces some basic control approaches used in robotic systems.

\subsection{Robot Manipulators}

A robot manipulator or a mechanical arm is one of the most important components in a robotic system. In our study, we consider the robotic systems that use multiple robotic manipulator to perform certain tasks. In this section, we introduce the basic components of robot manipulators, which serve as an important background to the later parts of the thesis. 
Links and Joints: Robot manipulators are composed of multiple links connected by joints into an open kinematic chain. There are two types of joints considered in this study, which are revolute and prismatic joints. A prismatic joint, such as hydraulic and pneumatic cylinder actuators allows a linear motion between two links. A revolute joint, such as a motor allows relative rotation between two links. In this study, we denote the joint variables as $q_{i}$, which represents the relative displacement between two links $l_{i}, l_{i+1}$.

Degrees of Freedom: The number of joints determines the degrees-of-freedom of the manipulator (This does not necessarily represent the degrees-of-freedom of the system, which depends on the configuration of each manipulator). Typically, a robotic system needs six degrees-of-freedom to control the position and orientation of the endeffector of a manipulator. A manipulator that has more than six links is referred to as a kinematically redundant manipulator.

Workspace: The workspace of a robot manipulator is the total area that the endeffector of a robot can reach. The workspace is depended on the structure of the robot manipulator and the mechanical constraint on each joint. For example, a typical servo motor may limit the movement of the joint to be less than 360 degrees. The workspace of robotic system can be divided into a reachable work space and a dextrous workspace. The reachable workspace is the entire set of point reachable by the system, and the dextrous workspace is those points where the manipulator can reach with arbitrary orientation. When an actuator failure occurs, the workspace of the robot is also reduced based on the characteristics of the failure. In this study, we only consider the reachable workspace of the system after the consideration of actuator failures.

Robot Configurations: By using different types of joints with different arrange- 
ments, the robot can be assembled into many configurations such as articulated configuration, spherical configuration, cylindrical configuration, etc. In this study, we consider the robotic system with articulated configuration and the Cartesian configuration. The articulated configuration or a revolute manipulator is the robot manipulator with multiple revolute joints such as an elbow type manipulator, which is one of the most common manipulator seen in literature. The Cartesian configuration is the robot manipulator with multiple prismatic joints. This type of configuration has the simplest dynamic of all configurations. The Cartesian configuration is used in our study as a benchmark system for testing the initial design of the actuator failure compensation framework.

\subsection{Euler-Lagrange Equation}

The Euler-Lagrange equation is one of the most important equations in developing a dynamic model of a robotic system. Because a robotic system is a mechanical system, it must follow the principle of conservation of energy. This means that if there is no external energy applied to the system, the summation of potential and kinetic energy of the system will always remain the same, regardless of how the system is changed. On the other hand, if we applied any external energy to the system, the change in potential and kinetic energy of the system will be equal to the amount of energy we put into the system.

One of the problems in deriving the dynamic model of a robot manipulator is the constraint force in the manipulator. Since each link in the robotic system is connected together, it creates holonomic constraints in the system. It is very difficult to measure or calculate the constraint force between each link, thus, the principle of virtual work is used in order to derive the dynamic model of the system. 
Definition of holonomic constraint: A constraint on $k$ coordinate $r_{1}, r_{2}, \ldots, r_{k}$ is called holonomic constraint if it is an equality constraint of the form

$$
g_{i}\left(r_{1}, \ldots, r_{k}\right)=0, i=1,2, \ldots, l
$$

where $l$ is the number of constraint equations of the system.

For a robot manipulator with $n$ links, the constraint equations can be written in terms of $n$ generalized coordinates $q_{1}, q_{2}, \ldots, q_{n}$, where $q_{1}, \ldots, q_{n}$ are all independent. In the case, each coordinate $r_{i}$ is a function of the generalized coordinate $r_{i}\left(q_{1}, \ldots, q_{n}\right), i=$ $1, \ldots, k$.

Consider the system in equilibrium, the net force on all particles in the system must be zero. This implies that the work done by each set of virtual displacement is zero as

$$
\sum_{i=1}^{k} F_{i}^{T} \delta r_{i}=0
$$

where $F_{i}$ is the total force (the sum of external forces and the constraint forces) on each particle $i$, and $\delta r_{i}$ is the virtual displacement of each particle. The virtual displacements are any set of infinitesimal displacement that satisfies the constraint equation (2.1).

Because the robotic system are not necessarily in an equilibrium state, we generalize the above equation using the D'Alembert's principle. The principle states that we can put any system into an equilibrium state by introducing the negative derivative of the momentum of the particle. With this, we replace $F_{i}$ in $(2.1)$ with $F_{i}-\dot{p}$ as

$$
\sum_{i=1}^{k} F_{i}^{T} \delta r_{i}-\sum_{i=1}^{k} \dot{p}_{i}^{T} \delta r_{i}=0
$$

By considering the fact that the total work done by the constraint force is zero, we 
can rewrite $(2.3)$ as

$$
\sum_{j=1}^{n} \Psi_{j} \delta q_{j}-\sum_{i=1}^{k} \dot{p}_{i}^{T} \delta r_{i}=0
$$

where

$$
\Psi_{j}=\sum_{i=1}^{k} f_{i}^{T} \frac{\delta r_{i}}{\delta q_{j}}
$$

with $f_{i}$ represent the external force done to each particle.

Define the kinetic energy of the system $K$ as

$$
K=\frac{1}{2} \sum_{i=1}^{k} m_{i}^{T} v_{i}^{T} v_{i}
$$

where the velocity $v_{i}=\dot{r}_{i}$. The momentum term in (2.3) can be written in the generalized coordinates as

$$
\sum_{i=1}^{k} \dot{p}_{i}^{T} \delta r_{i}=\sum_{j=1}^{n}\left(\frac{d}{d t} \frac{\partial K}{\partial \dot{q}_{j}}-\frac{\partial K}{\partial q_{j}}\right) \delta q_{j}
$$

By substituting (2.7) into (2.3), we have

$$
\sum_{j=1}^{n}\left(\frac{d}{d t} \frac{\partial K}{\partial \dot{q}_{j}}-\frac{\partial K}{\partial q_{j}}-\Psi_{j}\right) \delta q_{j}=0
$$

Because the coordinates $q_{1}, \ldots, q_{n}$ are independent, we have

$$
\frac{d}{d t} \frac{\partial K}{\partial \dot{q}_{j}}-\frac{\partial K}{\partial q_{j}}-\Psi_{j}=0
$$

for $j=1, \ldots, n$. For a robot manipulator, the generalized force $\Psi_{j}$ is the sum of the actuator torque $\tau_{j}$ and a potential field. Thus, $\Psi_{j}$ can be written as

$$
\Psi_{j}=\tau_{j}-\frac{\partial V}{\partial q_{j}}
$$


where $V$ is the potential energy of the system. The general form of the Euler-Lagrange equation can be written as

$$
\frac{d}{d t} \frac{\partial L}{\partial \dot{q}_{j}}-\frac{\partial L}{\partial q_{j}}=\tau_{j}
$$

where $L=K-V, K$ is the kinetic energy, $V$ is the potential energy of the system, $q_{j}$ is the generalized coordinates and $\tau_{j}$ is the actuator torque.

The dynamic model of a robotic system is usually written in the following form

$$
D(q) \ddot{q}+C(q, \dot{q}) \dot{q}+g(q)=\tau
$$

where $q \in R^{n}$ is the output of the system representing the angle of each joint, $D(q) \in$ $R^{n \times n}$ is the inertia matrix of the manipulators, $C(q, \dot{q}) \in R^{n \times n}$ is the Coriolis and centrifugal term, $g(q) \in R^{n}$ is the gravity term and the input is $\tau=\left[\tau_{1}, \ldots, \tau_{n}\right]^{T}$ with $\tau_{i}$ as the torque of each actuator.

\subsubsection{Example: Single-link Robotic Manipulator System}

In order to further our understanding of the Euler-Lagrange equation in deriving a dynamic model of a robotic system, we first consider a single-link robotic manipulator system as follows.

Let consider $q_{l}, q_{m}$ as the angles of a link and a motor in the system. $J_{m}, J_{l}$ as the moment of inertia of the motor and the link accordingly. We can see that the system will have its kinetic energy equation as follows

$$
K=\frac{1}{2} J_{m} \dot{q}_{m}^{2}+\frac{1}{2} J_{l} \dot{q}_{l}^{2}
$$

Now we consider the potential energy of the system. This potential energy will be depend on the position of the robot. Since we let $q_{m}$ be the angle of the system, we 
will need to consider the gear ratio in order to get the real angle of the link $q_{l}$. For $k$ represents the gear ratio of the robot arm, the potential energy is

$$
V=\operatorname{Mgl}\left(1-\cos \frac{\theta_{m}}{k}\right)
$$

With the potential energy and the kinetic energy of the system we can now get the total energy of the system. The Lagrangian $L$ is as

$$
L=\frac{1}{2}\left(J_{m}+\frac{J_{l}}{k^{2}}\right) \dot{q}_{m}^{2}+M g l\left(1-\cos \frac{q_{m}}{k}\right)
$$

As a result, we have the dynamic of a single-link robot manipulator system as

$$
J_{m}+\frac{J_{l}}{n} \ddot{q}_{m}+\frac{M g l}{n}\left(\sin \frac{q_{m}}{k}\right)=\tau
$$

\subsubsection{Example: Two-link Planar Elbow Robotic Manipulator}

In order to gain a better understanding of the dynamic model of robotic system, we expand our knowledge of Euler-Lagrange equation to the two-link planar elbow robotic manipulator. The two-link planar elbow robotic manipulator is one of the standard robotic system that can represent a class of robot manipulator.

The first step to obtain dynamics of the robot manipulator is to find the EulerLagrange equation for the system. In order to do so, we will need to get kinetic and potential energy of the system. We first define the parameters of the system as follows. Lets $q_{i}$ will be the angle of each joint, mass and length of each link will be represented as $M_{i}$, and $l_{i}$ accordingly. The distance from a joint to the center of mass of that joint will be $l_{c i}$. The kinetic energy is as

$$
v_{c i}=J_{c i} \dot{q}, i=1,2
$$




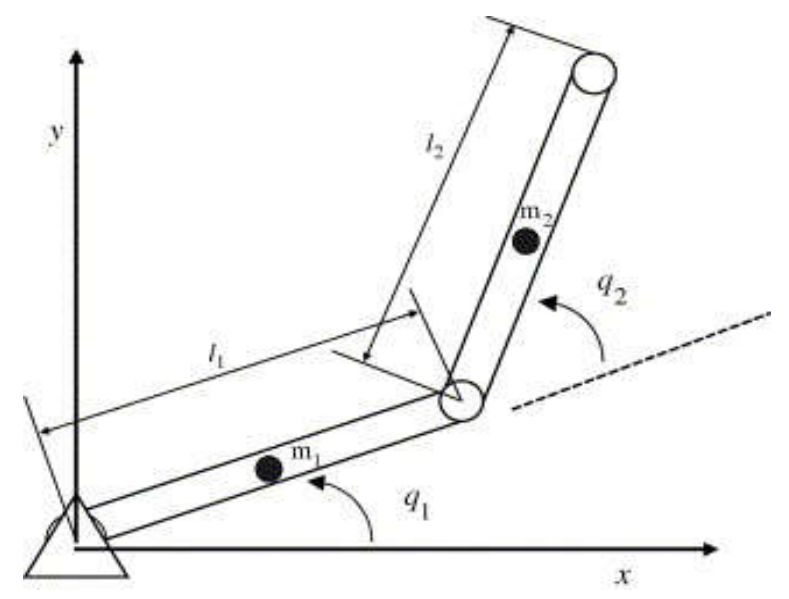

Figure 2.1: Two-link planar elbow robotic manipulator.

where $J_{c i}$ represents the Jacobian matrix of the system

$$
J_{c 1}=\left[\begin{array}{cc}
-l_{c 1} \sin q_{1} & 0 \\
l_{c 1} \cos q 1 & 0 \\
0 & 0
\end{array}\right]
$$

For the second link, we have the Jacobian matrix as

$$
J_{c 2}=\left[\begin{array}{cc}
-l_{1} \sin q_{1}-l c 2 \sin \left(q_{1}+q_{2}\right) & -l_{c 2} \sin \left(q_{1}+q_{2}\right) \\
l_{1} \cos q_{1}+l c 2 \cos \left(q_{1}+q_{2}\right) & l_{c 2} \cos \left(q_{1}+q_{2}\right) \\
0 & 0
\end{array}\right]
$$

For potential energy we have the summation of each link in the system

$$
V=V_{1}+V_{2}=\left(M_{1} l_{c 1}+M_{2} l_{c 2}\right) g \sin q+M_{2} l_{c 2} g \sin \left(q_{1}+q_{2}\right) .
$$

By substituting the kinetic and potential energy into the Euler Lagrange's equation, we can obtain the dynamic model of the system in the form The dynamic of the 
two-link planar system can be written in the form

$$
D(q) \ddot{q}+C(q, \dot{q}) \dot{q}+g(q)=\tau,
$$

where the inertia matrix $D(q)$ is as

$$
D(q)=M_{1} J_{c 1}^{T} J_{c 1}+M_{2} J_{c 2}^{T} J_{c 2}+\left[\begin{array}{cc}
I_{1}+I_{2} & I_{2} \\
I_{2} & I_{2}
\end{array}\right]
$$

The matrix $C(q, \dot{q})$ is

$$
C=\left[\begin{array}{cc}
\left(-M_{2} l_{1} l_{c 2} \sin q_{2}\right) \dot{q}_{1} & \left(-M_{2} l_{1} l_{c 2} \sin q_{2}\right)\left(q_{1}+q_{2}\right) \\
\left(M_{2} l_{1} l_{c 2} \sin q_{2}\right) \dot{q}_{1} & 0
\end{array}\right]
$$

The gravitational term $g(t)$ is

$$
g(q)=\left[\begin{array}{c}
\left(m_{1} l_{c 1}+m_{2} l_{c 1}\right) \cos q_{1} \\
m_{2} l_{c 2} \cos q_{1}+q_{2}
\end{array}\right]
$$

In this study, we focus on robotic system with redundancy such as cooperative manipulator parallel manipulators. Such system is a combination of multiple manipulators discussed in this section. With the dynamic model of robot manipulator, we can derive the dynamic model of the cooperative manipulator parallel manipulator systems to be used as the model for the proposed adaptive actuator failure compensation design. 


\subsection{Cooperative and Parallel Manipulators}

In this study, we mainly consider two types of robotic systems with redundancy. In this research, the cooperative manipulator robotic system is referred to many manipulators that is perform a single task together. For this type of system, we assume that the problem from the closed loop kinematic constraint is negligible because the base position of each manipulation or the gripping position of each manipulator can be moved, which enable some flexibility in the system structure. On the other hand, the parallel manipulator robotic system is considered a signal system that contains multiple manipulator in a parallel structure. In this case the manipulator and well as the gripping position of a manipulator is mounted at a specific location in the system. Thus, one will need to consider the system constrain for the development of an adaptive failure compensation for the parallel structure robotic system.

The general dynamic model of robotic systems considered in this study can be divided into two parts, which are the dynamic of each manipulator and the dynamic of the mutual object. In order to develop a controller for such systems, we need to obtain the combined dynamic of the system. In this section, we will develop a general dynamic model of the system with $n$ degrees of freedom and $m$ actuators. The combined dynamic model of the robotic systems can be described as follows.

$$
D_{c}\left(x_{o}\right) \ddot{x}_{o}+C_{c}\left(x_{o}, \dot{x}_{o}\right) \dot{x}_{o}+G_{c}\left(x_{o}\right)=E\left(x_{o}\right) \tau,
$$

where $x_{o} \in R^{n}$ is the output of the system representing the position and orientation of the mutual object, $D_{c}\left(x_{o}\right) \in R^{n \times n}$ is the combined inertia matrix of the object and manipulators, which is assumed to be a bounded and positive definite matrix, $C_{c}\left(x_{o}, \dot{x}_{o}\right) \in R^{n \times n}$ is the Coriolis and centrifugal term, $G_{c}\left(x_{o}\right) \in R^{n}$ is the gravity 
term, $E=\left[E_{1}, E_{2}, \ldots, E_{m}\right] \in R^{n \times m}$ is a transformation matrix from the joint space to the task space, $E_{i} \in R^{n}$ and $\tau=\left[\tau_{1}, \ldots, \tau_{m}\right]^{T}$ with $\tau_{i}$ as the torque of each actuator. Since the cooperative manipulator system need to carry various objects, some parameters in $D_{c}, C_{c}$ and $G_{c}$ are often unknown.

Although the parameters of the system are unknown, it is common to assume that the system satisfies the following structural properties:

a) $D_{c}$ is a symmetric and positive definite matrix.

b) $\dot{D}_{c}-2 C_{c}$ is a skew symmetric matrix.

These properties will be used for proving the negative definite property of matrices, which is important in the process of finding suitable Lyapunov function to ensure the performance of the system.

\subsection{Lyapunov Stability Theory}

The adaptive actuator failure compensation framework developed in this study utilizes the Lyapunov stability theory to ensure closed loop stability and boundedness of signals in the system. The Lyapunov stability theory is widely used in the analysis and design of various types of systems. The concept of stability in the sense of Lyapunov will be introduced in this section, the stability concept and the Barbalat's Lemma is also discussed.

Stability is one of the most important concepts in control theory. The concept of stability is related to the ability to remain in a state regardless of small disturbance. In order to introduce the definition of the stability, we consider a nonlinear dynamic of a non-autonomous system described by the differential equation as

$$
\dot{x}=f(x, t),
$$


where $x \in R^{n}$ and $f: R^{n} \times R_{+} \rightarrow R^{n}$. The definition of equilibrium point and its stability is defined as follows.

Definition 2.1: A state of the system $x_{e}$ is considered to be an equilibrium point if $f\left(x_{e}, t\right)=0$ for all $t>0$.

Definition 2.2: The equilibrium point $x_{e}$ is said to be stable if for every $\epsilon>0$ and any $t_{0} \geq 0$, there exist a $\delta(\epsilon, t)>0$ such that $\left\|x_{0}\right\|<\delta$ implies that $\left\|x\left(t ; x_{0}, t_{0}\right)\right\|<$ $\delta, \forall t>t_{0}$

Definition 2.3: The equilibrium point $x_{e}$ is said to be asymptotically stable if it is stable and for every $t_{0} \geq 0$, there exists a function $\rho\left(t_{0}\right)>0$ such that $\left\|x_{0}\right\|<\rho$ implies that $\lim _{t \rightarrow \infty}\left\|x\left(t ; x_{0}, t_{0}\right)\right\|=0$.

Lyapunov Stability Theorem: Given an autonomous system $\dot{x}=f(x)$, then the equilibrium point is stable if there exists a scalar function $V(x): R^{n} \rightarrow R$, such that - $V(x)=0$ if and only if $x=0$ and $V(x)>0$ if and only if $x \neq 0$.

- $\dot{V}(x) \leq 0$ for all value $x \neq 0$

The equilibrium point is asymptotically stable if the Lyapunov function satisfies $\dot{V}(x)<0$ for all value $x \neq 0$.

Barbalet's Lemma: Let $f(t)$ be a differentiable function, the Barbalet's lemma states that if $\lim _{\rightarrow} f(t)=k<\infty$ and if $\dot{f}(t)$ is uniformly continuous, then $\lim _{t \rightarrow \infty} f(t)=$ 0 . In the context of the Lyapunov stability analysis: If $V(x, t)$ is lower bounded, $\dot{V} \leq 0$ and $\ddot{V}$ is bounded, then we can conclude that $\dot{V} \rightarrow 0$ as $t \rightarrow \infty$. In this study, we often use the Lyapunov theory to prove that the tracking error $e$ goes to zero asymp- 
totically. In this case, we need $e \in L_{\infty} \cap L_{2}$ and $\dot{e}$ is bounded, so that $e \rightarrow 0$ as $t \rightarrow \infty$.

\subsection{Control of Robotic Systems}

In this section, we discuss control schemes in robotic system used in this study, which are the Slotine and Li' control design and the backstepping control design. The control schemes discuss in this section is designed for robotic system without actuator failure. The control schemes introduced in this section will be used as nominal control for each controller bank in the adaptive actuator failure compensation framework.

\subsubsection{Slotine and Li's Control Design}

Consider the dynamic model of the robotic system

$$
D_{c}(q) \ddot{q}+C_{c}(q, \dot{q}) \dot{q}+G_{c}(q)=E\left(x_{o}\right) \tau
$$

If we can completely generate $E\left(x_{o}\right) \tau$, it is possible to design the baseline controller using the Slotine and Li's control algorithm as follows.

Design for the known parameter case. If all parameters in the system are known, we can design a controller using the Slotine and Li's approach [18]. This controller is designed for the system without actuator failure. It will be used as a baseline for developing the adaptive actuator failure compensation scheme.

In this case, we first define an error vector $s=\dot{e}+\Lambda_{0} e$, where $e=x_{o}-x_{d}, x_{d}$ is the desired trajectory, $v=\dot{x}_{d}-\Lambda_{0}$ e being an intermediate vector signal and let $\Lambda_{0}$ be any $n \times n$ constant matrix whose eigenvalues have positive real parts. The definition 
of this error vector means that the convergence of $s$ also implies the convergence of the tracking error.

The cooperative manipulator model can be rewritten with the error vector as

$$
D_{c} \dot{s}+C_{c} s=E \tau-D_{c} \dot{v}-C_{c} v-G_{c} .
$$

Since we assume that all parameters are known and the rank of $E\left(x_{o}\right)$ is larger than $n$, we can always choose a controller such that

$$
E \tau(t)=D_{c} \dot{v}+C_{c} v+G_{c}-K_{d} s(t)
$$

where the gain matrix $K_{d}$ is chosen to be a positive definite matrix. If we can precisely generate $E \tau(t)$, the closed-loop system becomes

$$
D_{c} \dot{s}+C_{c} s+K_{d} s=0
$$

From closed loop system (2.30) and the definition of $s$, we can guarantee that the system output $x_{o}$ tracks the desired trajectory $x_{d}$ asymptotically. In this case, the controller needs complete knowledge of parameters in the system.

Design for the unknown parameters case. Since robotic systems often interact with the environment, some parameters in $D_{c}, C_{c}$ and $G_{c}$ in often unknown. To design a controller for the system with parameter uncertainties, we estimate the unknown matrices with $\hat{D}, \hat{C}, \hat{G}$ respectively. With this equation (2.29) becomes

$$
E \tau(t)=\hat{D} \dot{v}+\hat{C} v+\hat{G}-K_{d} s(t) .
$$


We can linearly parameterize the system by rewriting the right side of equation (2.31) as a known regressor matrix $Y\left(x_{o}, \dot{x}_{o}, v, \dot{v}\right)$ multiplied by an estimate $\hat{\theta}$ of an unknown parameter vector, the controller becomes

$$
E \tau=\hat{D} \dot{v}+\hat{C} v+\hat{G}-K_{d} s=Y \hat{\theta}-K_{d} s
$$

where the regressor matrix $Y\left(x_{o}, \dot{x}_{o}, v, \dot{v}\right)$ is such that

$$
D_{c} \dot{v}+C_{c} v+G_{c}=Y\left(x_{o}, \dot{x}_{o}, v, \dot{v}\right) \theta^{*}
$$

with $\theta^{*}$ being the unknown parameter vector in the system that is estimated by $\hat{\theta}$. The closed-loop system becomes

$$
D_{c} \dot{s}+C_{c} s+K_{d} s=Y \tilde{\theta}
$$

where $\tilde{\theta}=\hat{\theta}-\theta^{*}$ is the estimation error of the unknown parameters in the system.

We can choose the adaptive law for $\hat{\theta}$ as

$$
\dot{\hat{\theta}}=-\Gamma^{-1} Y^{T} s
$$

With the chosen adaptive law, we can prove that $s, \dot{s}$ is uniformly bounded and $s \in L^{2}$, so $\lim _{t \rightarrow \infty} s(t)=0$. From the definition of $s$, this also implies that the tracking error converges asymptotically.

\subsubsection{Backstepping Nonlinear Control Design}

In order to control a nonlinear system, we need to construct a nonlinear controller for the system. In this section, we consider using a backstepping design to develop a 
controller for a second-order nonlinear system. Consider the nonlinear system in the state space form as

$$
\begin{aligned}
\dot{x}_{1} & =f\left(x_{1}\right) x_{2}, \dot{x}_{2}=g\left(x_{1}, x_{2}\right)+u(t) \\
y & =x_{1},
\end{aligned}
$$

where $x_{1}, x_{2}$ is the state of the system, $y$ is the output and $u(t)$ is the input of the system. The objective is to design a state feedback control signal $u(t)$ such that all closed-loop signal is bounded and the system output tracks a given reference output signal $y_{m}(t)$. For a second order system, the backstepping design can be done in two steps as follows.

Step 1: Define $z_{1}=x_{1}-y_{m}, z_{2}=x_{2}-\beta$, where $\beta$ is a design function to be determined. From the state equation of the system, we can write $\dot{z}_{1}$ as

$$
\dot{z}_{1}=\dot{x}_{1}-\dot{y}_{m}=f\left(x_{1}\right) x_{2}-\dot{y}_{m}=f\left(x_{1}\right)\left(z_{2}-\beta\right)-\dot{y}_{m} .
$$

In order to choose the designed function $\beta$ to stabilize the system, we consider a Lyapunov candidate function and its derivative as

$$
\begin{aligned}
& V_{1}=\frac{1}{2} z_{1}^{T} z_{1} \\
& \dot{V}_{1}=z_{1}\left(f\left(x_{1}\right) z_{2}+f\left(x_{1}\right) \beta-\dot{y}_{m}\right) .
\end{aligned}
$$

With the design function

$$
\beta=f\left(x_{1}\right)^{-1}\left(-c_{1} z_{1}+\dot{y}_{m}\right),
$$


where $c_{1}$ is a positive constant. The derivative of the Lyapunov function becomes

$$
\dot{V}_{1}=-c_{1} z_{1}^{T} z_{1}+f\left(x_{1}\right) z_{1} z_{2}
$$

We see that the term $-c_{1} z_{1}^{T} z_{1}$ is always negative.

Step 2: Since $z_{2} \neq 0$, we continue using backstepping design by considering

$$
\dot{z_{2}}=\dot{x}_{2}-\dot{\beta}=g\left(x_{1}, x_{2}\right)+u(t)-\dot{\beta},
$$

and the second Lyapunov function

$$
V_{2}=V_{1}+\frac{1}{2} z_{2}^{T} z_{2}
$$

The time-derivative of $V_{2}$ is

$$
\dot{V}_{2}=\dot{V}_{1}+z_{2}^{T} \dot{z}_{2}=-c_{1} z_{1}^{T} z_{1}+z_{2}^{T}\left(f\left(x_{1}\right) z_{1}+g\left(x_{1}, x_{2}\right)+u(t)-\dot{\beta}\right)
$$

As a result, the control signal $u(t)$ is chosen as

$$
u(t)=-g\left(x_{1}, x_{2}\right)-c_{2} z_{2}-f\left(x_{1}\right) z_{1}+\dot{\beta}
$$

With the chosen $u(t)$, equation (2.44) becomes

$$
\dot{V}_{2}=-c_{1} z_{1}^{T} z_{1}-c_{2} z_{2}^{T} z_{2}
$$

With a positive constant $c_{2}$, we have $\dot{V}_{2}$ is negative definite. With this design, the nonlinear can guarantee the closed-loop signal boundedness and asymptotic output tracking of the system: $\lim _{t \rightarrow \infty}\left(y(t)-y_{m}(t)\right)=0$. 
Remark 2.1: The adaptive actuator failure compensation framework developed in this study enable the conventional control design such as the Slotine and Li's design or the backstepping control design to be able to compensate for actuator failures in the system. In this study, we will use the backstepping control approach for the system with known parameters and the Slotine and Li design for the system with unknown parameters.

From the derivation using the backstepping technique, we need to design the adaptive actuator failure compensation to ensure $D_{c}^{-1} E\left(x_{1}\right) \tau=-D_{c}^{-1}\left(-C_{c}-G_{c}\right) x_{2}-$ $c_{2} z_{2}-z_{1}+\dot{\beta}$. If all parameters of the system are known, we can directly design actuator failure compensation scheme with this algorithm. The cancellation of the nonlinear terms in the backstepping control design could improve the system transient response of the adaptive actuator failure compensation scheme. The disadvantage of this approach is that the controller structure needs the inverse of the inertia matrix $D_{c}^{-1}$, which may be difficult to obtain, especially for a robotic system with parameter uncertainties. 


\section{Chapter 3}

\section{Problem Formulation}

The overall control problem for robotic system can be described as follows, provided a desired trajectory of the end-effector, the dynamic model of the system, finds a control law that produce force or torque signals to each actuator in the system, so that the system operate according to the desired performance. The control design for a robot manipulator can be divided into two steps. First, a robotic end-effector travel path is first specified. From the movement trajectory of the end-effector and also via resorting to the inverse kinematics, the motions of each joint can therefore be determined so as to create the desired trajectory for the end-effector. The next step is to figure out the amount of torque that one has to apply to joints so that the joints are able to achieve the desired motion. The torque can be calculated based on the dynamic model of the system (Kinematic control approach).

Controlling the robotic manipulator to conduct in a designated fashion is a difficult task because the dynamic of a robotic system is extremely nonlinear. Regarding robotic systems, the parameter related to the joints and payload may be unknown or may change during the operation. For the purpose of having a higher repeatability and accuracy in the robotic systems performance, we need to consider the dynamic characteristic of the system. Traditional control techniques treat a robotic mechanism as uncoupled linear subsystems, which can achieve adequate performances at low speeds. However, for applications that require high-speed actions, they are no longer 
effective. In order to solve this problem, the use of advanced control algorithm such as adaptive control is needed (Dynamic control approach).

Adaptive control is able to adjust parameters in the system to match unknown parameters, or parameters with uncertainties. With respect to the adaptive control, the controller does not have to rely on the exact data of parameters in the system, and if there are some changes such as the change of parameter values or actuator failure, the controller is able to handle it through an adaptation algorithm.

The objective of this research is to develop an adaptive actuator failure compensation scheme to ensure closed-loop stability and asymptotic output tracking of robotic systems with parameter uncertainties in the presence of uncertain actuator failures. In this chapter, we discuss the fundamental problems that are needed to be solved as well as the step by step approach of this research for developing the desired actuator failure compensation framework.

\subsection{Actuator Failures in Robotic Systems}

Actuator failures in control systems may cause severe system performance deterioration and can even lead to system instability. In order to improve the reliability and safety of the system, the changes from such failure must be accommodated. In this research, the adaptive control framework needs to be able to compensate for actuator failures in the system without exact knowledge of the actuator failure values, failure time, or the failure pattern.

\subsubsection{Actuator Failure Uncertainties}

In general, a robotic system needs to operate in an unknown or even hazard environment. Under such extreme conditions, a robotic system is often disturbed and parts 
of the system can be easily damaged. The problem such as the damage directly to actuator such as overheat, structural damage and electrical problem, or the indirect damage such as communication problems and software malfunctions are considered an actuator failure in the system. From such problem, we can see that the actuator failures can be uncertain, that is, it is not known when, in what manner, or how many actuators fail at any point during the system operation. The actuator failures occur with various characteristic, such as some unknown inputs may be stuck at some fixed values at unknown time instants, or the actuator could completely fail and the control input becomes zero. In any case, unlike normal disturbances to the system, actuator failures cause additional and large system uncertainties. For example, when a motor in a robotic system fails, the system structure from the active input of the motor to the output of the end-effector experiences significant changes, and so do the system parameters. Moreover, the characteristic of the failure changes significantly depending on the joint location of the failed motor. Because of this reason, the compensation of the unknown failures in the system is a challenging problem for robotic system. In order to solve this problem, a complete adaptive actuator failure compensation framework is needed to design a controller for the system.

\subsubsection{Actuator Failure Model}

In this subsection, we define a mathematical description of actuator failure in the system as well as model the characteristic of actuator failure value. Let $\tau(t)$ be the system input vector. When an actuator failure occurs, the control input component $\tau_{i}(t)$ associated with the failing actuator $i$ becomes an arbitrary signal, which can be expressed as

$$
\tau_{i}(t)=\bar{\tau}_{i}(t), t \geq t_{i}, i \in\{1,2, \ldots, m\},
$$


where $m$ is the number of actuators in the system.

Although we do not know the exact value of the control input when the failure occurs, we can model the failure based on knowledge of the actuator structure as

$$
\bar{\tau}_{i}(t)=\bar{\tau}_{i 0}+\sum_{j=1}^{\bar{\rho}} \bar{\tau}_{i j} f_{a i j}(t), t \geq t_{i}
$$

where $\bar{\rho}$ is the number of components in the failing signal, $f_{a i j}$ are known functions corresponding to each component of the actuator and $i, t_{j}, \bar{\tau}_{i j}$ are unknown failure index, failure time and failure value of each actuator component.

With some specifications of the unknown constants $\bar{\tau}_{j 0}, \bar{\tau}_{i j}$ and known functions $f_{a i j}(t)$, we can model most common actuator failures such as the lock in-place failure of the actuator: $\bar{\tau}_{j}=\bar{\tau}_{j 0}$, which may happen when the controller fails to communicate with the actuator, or the complete failure of a motor in the system: $\bar{\tau}_{j}=0$. Additionally, the terms $\bar{\tau}_{i j} f_{a i j}(t)$ in the actuator failure model can be used to model additional types of time varying failures such as a square wave actuator failure, which the failure happens periodically.

\subsubsection{Actuator Failure Pattern}

Because the behavior of the system with failures also changes according to which and how many actuators fail at any given time. The concept of actuator failure pattern which describe the configuration of actuator failure is discussed in this section. In this study, we define actuator failures in the system as follows: Let $\Sigma$ be a set of all possible actuator failure pattern such that $\Sigma=\left\{\sigma_{(1)}, \sigma_{(2)}, \ldots, \sigma_{(N)}\right\}$, where $N$ is the number of all possible actuator failure pattern that satisfies Assumption 3.1, and $\sigma_{(i)}=\operatorname{diag}\left\{\sigma_{(i) 1}, \sigma_{(i) 2}, \ldots, \sigma_{(i) m}\right\}$ is the actuator failure pattern with $\sigma_{(i) j}=1$ if actuator $j$ fails and $\sigma_{(i) j}=1$ otherwise. 
Assumption 3.1: Define a matrix $\bar{E}_{(i)}$ from the dynamic model of the robotic system (2.28) for each actuator failure pattern $\sigma_{(i)}$, as a submatrix of $E$ without the columns associated with the failing actuator (each associated column $j$ is such that $\sigma_{(i) j}=1$ ) . In this study, we assume that the rank of the matrix $\bar{E}_{(i)}$ must be larger or equal to the degree of freedom of the system for every possible actuator failure pattern. $\quad \nabla$

\subsection{Basic Problem Statements}

As we can see from the literature review, the development of adaptive actuator failure compensation scheme is still in an early stage and there are several open problems that are needed to be answered. In order to develop a complete adaptive actuator failure compensation framework for robotic system, we divided the study into two major stages, each solving different critical issues as follows. During the first stage of the development, the objective of the research is to lay out fundamental ideas and techniques needed for the adaptive actuator failure compensation framework. At this stage, we consider the robotic system with known parameters and development of the framework is focused entirely on the actuator failure compensation scheme. After obtaining the initial framework, the second stage of the development is to extend the framework to a more general class of the robotic system. At this stage, we have solved several key technical issues in control and robotic ficlds such as the uncertainties in the system as well as the closed loop kinematics constraint in the robotic system with redundancy.

\subsubsection{Initial Stage of the Development}

In the initial stage of the development of our adaptive actuator failure compensation framework, the research can be divided into two steps. In the first step, we design 
an adaptive actuator failure compensation scheme for a theoretical benchmark to ensure the feasibility of the proposed control scheme. In the second step, we apply the control design to a more practical system. In this case, we apply our control scheme to a Hexapod system, which is one of the most important classes of robotic systems with redundancy.

\section{Step 1: Design for a Cooperative Manipulator System}

In this step, we develop an adaptive actuator failure compensation control framework for cooperative manipulator in the presence uncertain actuator failures. In this step, our aims is to solve the following problems:

- Solve an open problem by developing an adaptive actuator failure compensation scheme for a cooperative manipulator system subject to uncertain actuator failure without explicit knowledge of the failing actuator.

- Develop an initial control design framework for the adaptive actuator failure compensation control scheme for a class of robotic systems.

The adaptive control scheme is applicable to the cooperative manipulator robotic system model of in the state space form as

$$
\begin{aligned}
& \dot{x}_{1}=x_{2} \\
& \dot{x}_{2}=g_{1}\left(x_{1}, x_{2}\right)+g_{2}\left(x_{1}, x_{2}\right) u(t)
\end{aligned}
$$

where $x_{1}=x_{o} \in R^{n}$ and $x_{2}=\dot{x}_{o} \in R^{n}$, are the states of the system, $y=x_{1} \in R^{n}$ is the output of the system, $g_{1} \in R^{n}, g_{2} \in R^{n x m}$ are known matrices based on the dynamic system, $n$ is the degree of freedom, $m$ is the number of the actuators, and $u \in R^{m}$ is the system input. Due to possible actuator failures, an applied feedback 
control signal $v(t)=\left[v_{1}, v_{2}, \ldots, v_{m}\right]^{T} \in R^{m}$ may not reach the system, as $u_{j}(t)$ is not equal to $v_{j}(t)$ if $u_{j}(t)=\bar{u}_{j}(t)$. More precisely, in the presence of actuator failures, the signal $u(t)$ is $u(t)=(I-\sigma(t)) v(t)+\sigma(t) \bar{u}$, where $\sigma(t)=\operatorname{diag}\left\{\sigma_{1}, \sigma_{2}, \sigma_{3}\right\}$ is the actuator failure pattern matrix such that $\sigma_{i}(t)=1$ if the $i$ actuator fails, that is, if $u_{j}(t)=\bar{u}_{j}(t)$, and $\sigma_{i}(t)=0$ otherwise, and $\bar{u}(t)=\left[\bar{u}_{1}, \ldots, \bar{u}_{m}\right]^{T}$.

Control Objective: The control objective is to design a feedback control signal $v(t)$ such that $\lim _{t \rightarrow \infty}\left(g_{2}\left(x_{1}\right) u(t)-\omega_{d}(t)\right)=0$, despite the uncertain actuator failures $u(t)=(I-\sigma(t)) v(t)+\sigma(t) \bar{u}$, where $\omega_{d}$ is a desirable control signal for $\omega$ to control the system model: $\dot{x}_{1}=x_{2}, \dot{x}_{2}=g_{1}\left(x_{1}, x_{2}\right)+\omega$, to make all signals in the closedloop system are bounded and the output $y(t)=x_{1}(t)$ asymptotically tracks a given reference signal $y_{m}(t)$. When this objective is met, the real system $\dot{x}_{1}=x_{2}, \dot{x}_{2}=$ $g_{1}\left(x_{1}, x_{2}\right)+g_{2}\left(x_{1}\right) u(t)$ will be ensured to have the desired signal boundedness and tracking properties, and this will be achieved through control adaptation.

The adaptive actuator failure compensation scheme is designed to compensate for actuator failure in the system without the knowledge of the failing actuator $j$, time that actuator failure occurs $t_{j}$, or value of the output of the failing actuator $\bar{u}_{j}(t)$, so that the system will remain stable and the output $y(t)$ asymptotically tracks the reference signal $y_{m}(t)$ even when actuator failures occur.

\section{Step 2: Design for a Hexapod System}

In this step, we extend the adaptive actuator failure compensation scheme to a Hexapod system. Our aims for this step of our development are as follows:

- Develop an adaptive actuator failure compensation scheme for a Hexapod subjected to uncertain actuator failure.

- Extend the scope of the adaptive actuator failure control design method to actuator failure cases where more than one actuator fails at the same time. 
- Study the simulation of the adaptive actuator failure compensation scheme based on the Hexapod model subject to uncertain actuator failure.

In this study, we will develop an adaptive actuator failure compensation control solution framework of a Hexapod in the presence of uncertain actuator failures. We consider the following Hexapod system.

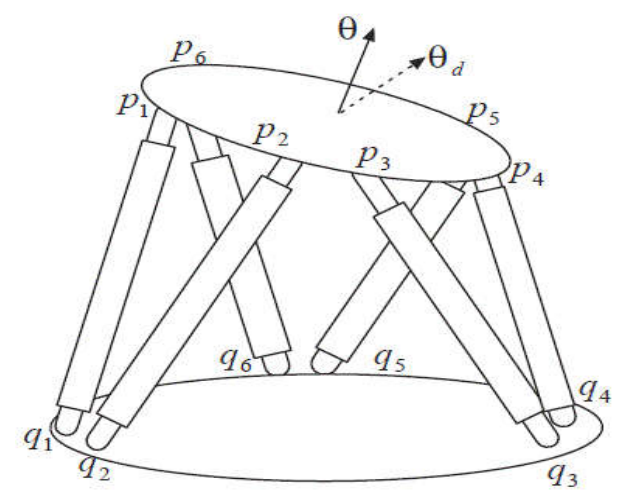

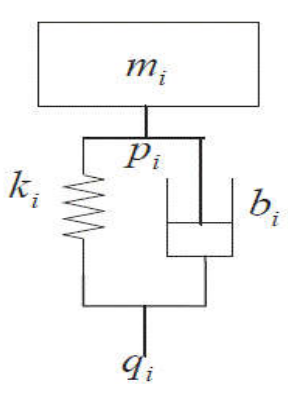

(a)

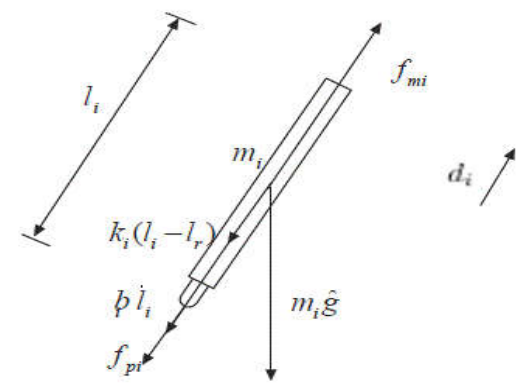

(b)

Figure 3.1: The model of Hexapod and the manipulator structure.

The Hexapod model (5.5) can be written in the state space form as

$$
\dot{x}_{1}=x_{2}, \quad \dot{x}_{2}=g_{1}\left(x_{1}, x_{2}\right)+g_{2}\left(x_{1}, x_{2}\right) u(t),
$$

where $x_{1}=\theta \in R^{3}$ and $x_{2}=\dot{\theta} \in R^{3}$ are the states of the system, $y=x_{1} \in R^{3}$ is the output of the system, $u=f_{m} \in R^{6}$ is the system input, $g_{1} \in R^{3}$, and $g_{2} \in R^{3 \times 6}$ are known matrices.

Control Objective: Consider the Hexapod model (3.4), due to possible actuator failures, an applied feedback control signal $v(t)=\left[v_{1}, v_{2}, \ldots, v_{6}\right]^{T}$ may not reach the system, as $u_{j}(t)$ is not equal to $v_{j}(t)$. More precisely, in the presence of failures, the 
signal $u(t)$ is

$$
u(t)=(I-\sigma(t)) v(t)+\sigma(t) \bar{u},
$$

where $\bar{u}$ is an unknown failure signal and $\sigma(t)=\operatorname{diag}\left\{\sigma_{1}, \sigma_{2}, \ldots, \sigma_{6}\right\}$ is the actuator failure pattern matrix such that $\sigma_{i}(t)=1$ if the $i$ actuator fails and $\sigma_{i}(t)=0$ otherwise.

The control objective is to design a feedback control signal $v(t)$ such that $\lim _{t \rightarrow \infty}\left(g_{2}\left(x_{1}, x_{2}\right) u(t)-\omega_{d}(t)\right)=0$, despite the uncertain actuator failures $u(t)=$ $(I-\sigma(t)) v(t)+\sigma(t) \bar{u}$, where $\omega_{d}$ is a desirable control signal for $\omega$ to control the system. In this case, we also allow concurrent actuator failure for up to three actuator failures in the system.

\subsubsection{Final Stage of the Development}

In this stage of the development of our adaptive actuator failure compensation framework, the research can be divided into three steps. In the first step, we design an adaptive actuator failure compensation scheme for a theoretical benchmark with parameter uncertainties. In the second step, we apply the control design to a more practical system, which is the

\section{Step 1: Design for a Benchmark System with Parameter Uncertainties}

In this step, we develop an adaptive actuator failure compensation scheme for a cooperative manipulator robotic system. This topic solves the following problems:

- Develop adaptive actuator failure compensation scheme for a class of cooperative manipulator system with parameter uncertainties, in addition to actuator failure uncertainties. 
- Derive a complete proof and perform performance analysis of the proposed actuator failure compensation scheme.

- Develop a compensation scheme for a square-wave actuator failure signal in a cooperative manipulator system.

- Study the simulation of the adaptive actuator failure compensation scheme based on a benchmark cooperative manipulator system with parameter uncertainties.

In this research, the cooperative manipulator robotic system is referred to many manipulators that is performing a single task together. For this type of system, we assume that the problem of system constraint is negligible because the base position of each manipulation or the gripping position of each manipulator can be moved, which enable some flexibilities in the system structure.

In this study, we will develop an adaptive actuator failure compensation scheme for a general cooperative manipulator system with $n$ degrees of freedom and $m$ actuators. The combined dynamic model of the cooperative systems balancing a rigid platform can be described as follows.

$$
D_{c}\left(x_{o}\right) \ddot{x}_{o}+C_{c}\left(x_{o}, \dot{x}_{o}\right) \dot{x}_{o}+G_{c}\left(x_{o}\right)=E\left(x_{o}\right) \tau,
$$

where $x_{o} \in R^{n}$ is the output of the system representing the position and orientation of the mutual object, $D_{c}\left(x_{o}\right) \in R^{n \times n}$ is the combined inertia matrix of the object and manipulators, which is assumed to be a bounded and positive definite matrix, $C_{c}\left(x_{o}, \dot{x}_{o}\right) \in R^{n \times n}$ is the Coriolis and centrifugal term, $G_{c}\left(x_{o}\right) \in R^{n}$ is the gravity term, $E=\left[E_{1}, E_{2}, \ldots, E_{m}\right] \in R^{n \times m}$ is a transformation matrix from the joint space to the task space, $E_{i} \in R^{n}$ and $\tau=\left[\tau_{1}, \ldots, \tau_{m}\right]^{T}$ with $\tau_{i}$ as the torque of each actu- 
ator. Since the cooperative manipulator system need to carry various object, some parameters in $D_{c}, C_{c}$ and $G_{c}$ are often unknown.

Control Objective: Our control objective is to ensure the orientation of the platform, or to make $x_{o}(t)$ tracks a desired trajectory $x_{d}(t)$ asymptotically. Consider the cooperative manipulator robotic model (6.1), due to uncertain actuator failures, an applied feedback control signal $\tau_{c}(t)=\left[\tau_{c 1}, \tau_{c 2}, \ldots, \tau_{c m}\right]^{T}$ may not reach the system, as $\tau_{i}(t)$ is not equal to $\tau_{c i}(t)$. More precisely, the signal $\tau(t)$ is $\tau(t)=$ $(I-\sigma(t)) \tau_{c}(t)+\sigma(t) \bar{\tau}$, where $\bar{\tau}$ is an unknown vector of the actuator failure values and $\sigma(t)=\operatorname{diag}\left\{\sigma_{1}, \sigma_{2}, \ldots, \sigma_{m}\right\}$ is the matrix of actuator failure pattern such that $\sigma_{i}(t)=1$ if the $i$ actuator fails and $\sigma_{i}(t)=0$ otherwise.

The control objective is to design a feedback control signal $\tau_{c}(t)$ such that the closed-loop system $D_{c} \dot{s}+C_{c} s+K_{d} s=0$, despite the uncertain actuator failures $\tau(t)=(I-\sigma(t)) \tau_{c}(t)+\sigma(t) \bar{\tau}$, where the error vector $s$ is defined as $s=\dot{e}+\Lambda_{0} e$ with $e=x_{o}-x_{d}, x_{d}$ is the desired trajectory, $\Lambda_{0} \in R^{n \times n}$ is a design matrix whose eigenvalues have positive real parts and the gain matrix $K_{d}$ is chosen to be positive definite. This control objective ensures the desired signal boundedness and tracking properties of the system.

\section{Step 2: Design for a 2-DOF Redundantly Actuated Parallel Manipulator}

In this step, we develop a new adaptive actuator failure compensation scheme for a parallel robotic system with parameter uncertainties. The objective of the control design is to ensure the desired closed-loop stability and asymptotic output tracking of the system subject to uncertain actuator failure. The goals this step are as follows:

- Develop an adaptive actuator failure compensation scheme for a parallel manipulator system with parameter uncertainties, in addition to actuator failure uncertainties. 
- Study the dynamic and control of parallel structure robotic manipulator and demonstrate how to deal with the constraint of the parallel structure.

- Clarification of several key technical issues and solutions for the adaptive actuator failure compensation scheme for parallel manipulator system.

- Study the simulation of the adaptive actuator failure compensation scheme based on a 2-DOF redundantly actuated parallel manipulator subject to uncertain actuator failure.

In this case, we consider a 2-DOF redundantly actuated parallel manipulator as our benchmark system. The parallel manipulator is actuated by three servo motors located at the bases A1, A2, and A3. The end-effector is mounted at the common joint $\mathrm{O}$, where the three manipulators meet. The joint angles are defined as follows: $q_{a 1}, q_{a 2}, q_{a 3}$ refer to the active joint angles, and $q_{b 1}, q_{b 2}, q_{b 3}$ refer to the passive joint angles.

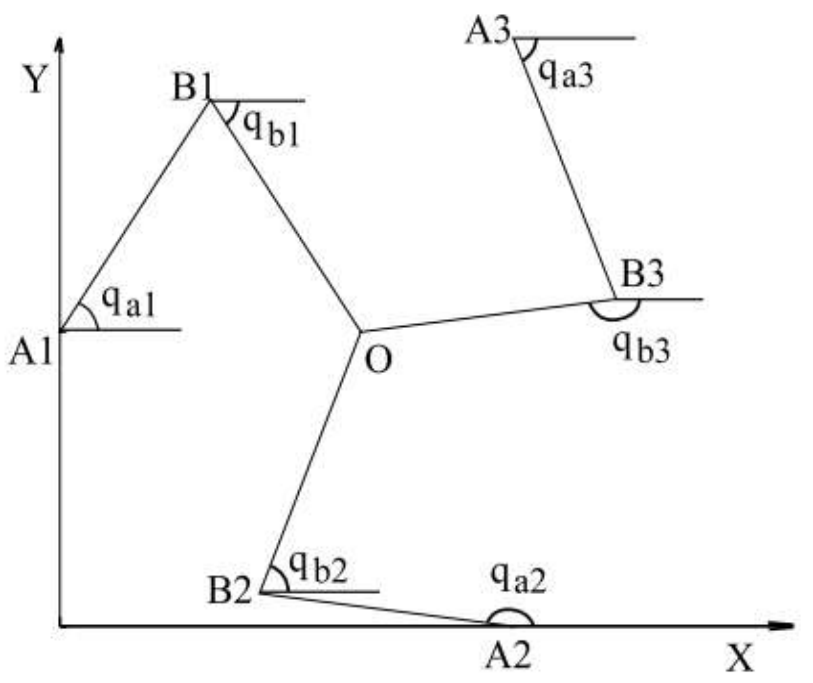

Figure 3.2: The 2-DOF redundantly actuated parallel manipulator.

The combined dynamic of the system with the closed-loop constraints can be 
expressed in the joint space as

$$
D_{c} \ddot{q}+C_{c} \dot{q}+f=\tau+A^{T} \lambda
$$

where $D_{c} \in R^{6 \times 6}$ is the combined inertia matrix, $C_{c} \in R^{6 \times 6}$ is the combined Coriolis and centrifugal matrix, $\tau=\left[\tau_{a 1}, \tau_{a 2}, \tau_{a 3}, 0,0,0\right]^{T}$, is the torque vector, $f=$ $\left[f_{a 1}, f_{a 2}, f_{a 3}, 0,0,0\right]^{T}$, is the friction vector and the vector $q=\left[q_{a 1}, q_{a 2}, q_{a 3}, q_{b 1}, q_{b 2}, q_{b 3}\right]^{T}$ represents the angle of each manipulator joint in the system.

Control Objective: Our control objective is to guarantee the position of the endeffector of the 2-DOF redundantly actuated parallel manipulator, or $q_{o}(t)$ tracks a desirable trajectory $q_{d}(t)$ asymptotically even when an actuator failure occurs. Consider the parallel manipulator model (7.9), due to possible actuator failures, an applied feedback control signal $\tau_{c}(t)=\left[\tau_{c 1}, \tau_{c 2}, \tau_{c 3}\right]^{T}$ may not reach the system, as $\tau_{a i}(t)$ is not equal to $\tau_{c i}(t)$. More precisely, in the presence of failures, the signal $\tau_{a}(t)$ is

$$
\tau_{a}(t)=(I-\sigma(t)) \tau_{c}(t)+\sigma(t) \bar{\tau},
$$

where $\bar{\tau}$ is an unknown failure vector and $\sigma(t)=\operatorname{diag}\left\{\sigma_{1}, \sigma_{2}, \sigma_{3}\right\}$ is the actuator failure pattern matrix such that $\sigma_{i}(t)=1$ if the $i$ actuator fails and $\sigma_{i}(t)=0$ otherwise.

The control objective is to design a feedback control signal $\tau_{c}(t)$ such that the closed-loop system $E^{T} D_{c} E \dot{s}+E^{T}\left(D_{c} \dot{E}+C_{c} E\right) s+K_{d} s=0$, despite the uncertain actuator failures $\tau_{a}(t)=(I-\sigma(t)) \tau_{c}(t)+\sigma(t) \bar{\tau}$, where the error vector $s$ is defined as $s=\dot{e}+\Lambda_{0} e$ with $e=q_{o}-q_{d}, q_{d}$ is the desired trajectory of the end-effector, $\Lambda_{0} \in R^{2 \times 2}$ is a design matrix whose eigenvalues have positive real parts and the gain matrix $K_{d}$ is chosen to be positive definite. When this objective is met, the system will be ensured to have the desired signal boundedness and tracking properties. 


\section{Step 3: Design for a Landing of Helicopter with Robotic Legs}

In this step, we develop an adaptive actuator failure compensation scheme for a helicopter landing system with parameter uncertainties. The aims of this step are:

- Develop an adaptive actuator failure compensation scheme for landing of a Helicopter with parameter uncertainties, in addition to actuator failures.

- Study the dynamic and control for the landing of a helicopter using robotic legs, and demonstrate how to deal with the constraint of the parallel structure.

- Clerify several key technical issues and solutions for the adaptive actuator failure compensation scheme for helicopter landing system and extend the failure compensation scheme to concurrent actuator failure case.

- Study the simulation of the adaptive actuator failure compensation scheme based on a Helicopter landing system subject to uncertain actuator failure.

In this study, we will develop an adaptive actuator failure compensation scheme for a helicopter landing system on uneven terrains with redundantly actuated parallel manipulator.

The dynamic model of the system can be expressed as

$$
E^{T} D_{m} E \ddot{q}_{o}+E^{T}\left(D_{m} \dot{E}+C_{m} E\right) \dot{q}_{o}+E^{T} g+J_{b} D_{o} \ddot{q}_{o}+J_{b} C_{o} \dot{q}_{o}=E^{T} \tau
$$

where $q_{o} \in R^{3}$ is the height, roll and pitch of the helicopter body, $D_{m} \in R^{12 \times 12}$ is the combined inertia matrix of the manipulator, $C_{m} \in R^{12 \times 12}$ is the combined Coriolis and centrifugal matrix, $\tau$ is the torque vector, matrix $E$ is a Jacobian matrix of the system. 


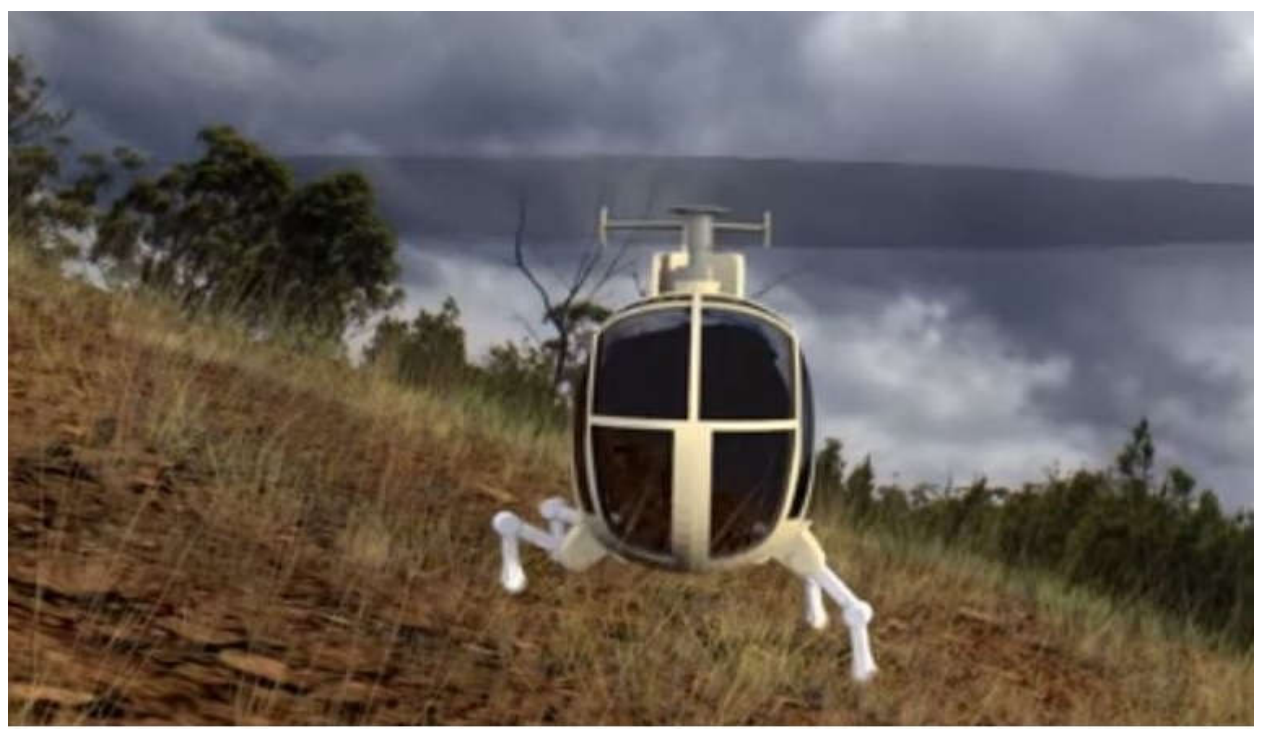

Figure 3.3: Landing of a helicopter with robotic legs on uneven terrains with redundantly actuated parallel manipulators.

The adaptive actuator failure compensation scheme developed in this study utilizes the redundancy from the parallel structure of the landing mechanism to compensate for possible actuator failures. In order to achieve this objective, the helicopter landing system considered in this study requires at least 3 functioning actuators in different manipulator, thus, we allows actuator failures for up to 3 different manipulators in each actuator failure case.

Control Objective: Our control objective is to guarantee the height displacement, roll angle and pitch angle of the helicopter body, or $q_{o}(t)$ tracks a desirable trajectory $q_{d}(t)$ asymptotically even when an actuator failure occurs. Consider the combined system model (8.6), due to possible actuator failures, an applied feedback control signal $\tau_{c}(t)$ may not reach the system, as $\tau_{j}(t)$ is not equal to $\tau_{c j}(t)$. More precisely, for each actuator failure pattern $\sigma_{(i)}$, the signal $\tau(t)$ is $\tau(t)=\left(I-\sigma_{(i)}(t)\right) \tau_{c}(t)+\sigma_{(i)}(t) \bar{\tau}_{(i)}$, where $\bar{\tau}_{(i)}$ is an unknown failure vector for the actuator failure pattern $\sigma_{(i)}$ case, and $\sigma_{(i)}(t)$ is the actuator failure pattern matrix such that $\sigma_{(i) j}(t)=1$ if the $j$ actuator 
fails and $\sigma_{(i) j}(t)=0$ otherwise.

The control objective is to design a feedback control signal $\tau_{c}(t)$ such that the closed-loop system satisfies: $E^{T} D_{m} E \dot{s}+E^{T}\left(D_{m} \dot{E}+C_{m} E\right) s+J_{b} D_{o} \dot{s}+J_{b} C_{o} s+K_{d} s=0$ in the nominal case, and also asymptotically in the adaptive case of unknown system parameters, despite the uncertain actuator failures $\tau(t)=\left(I-\sigma_{(i)}(t)\right) \tau_{c}(t)+\sigma_{(i)}(t) \bar{\tau}$, where the error vector $s$ is defined as $s=\dot{e}+\Lambda_{0} e$ with $e=q_{o}-q_{d}, q_{d}$ is the desired trajectory of the end-effector, $\Lambda_{0} \in R^{2 \times 2}$ is a design matrix whose eigenvalues have positive real parts and the gain matrix $K_{d}$ is chosen to be positive definite. When this objective is met, the system will be ensured to have the desired signal boundedness and tracking properties $\lim _{t \rightarrow \infty} e(t)=0$.

We will present each adaptive actuator failure compensation design in the following chapter, where the initial stage of the development is presented in Chapter 4 and 5, and the second stage with unknown parameters is presented in Chapter $6-8$. 


\section{Chapter 4}

\section{Design for A Cooperative Manipu-}

\section{lator System}

In this chapter, we design an adaptive actuator failure compensation scheme to control a benchmark cooperative manipulator robotic system. The design ensures desired closed-loop stability and asymptotic output tracking of the system subject to uncertain actuator failure. In this case, we assume that all parameters in the system are known.

Cooperative manipulator robotic systems play a critical role in many projects such as search and rescue operations, space explorations, and underwater missions. In order to improve the reliability of the system, we need a controller that can achieve certain desired properties even when actuator failure occurs. In this chapter, we develop a new adaptive actuator failure compensation scheme to control a cooperative manipulator robotic system. This study aim is to solve the following problems:

- Solve an open problem by developing an adaptive actuator failure compensation scheme for a cooperative manipulator system subject to uncertain actuator failure without explicit knowledge of the failing actuator.

- Develop an initial control design framework for the adaptive actuator failure compensation control scheme for a class of robotic systems. 
The chapter is organized as follows. In Section II, we formulate the problem with the discussion of the actuator redundancy, control objective, and actuator failure model. In Section III, the dynamic model of a cooperative robotic system is developed using the Euler-Lagrange equation. In Section IV, we design a nominal controller for the example system in a nominal situation in which the knowledge of actuator failure is known, to construct the controller structure and parameterization for failure compensation. In Section V, the adaptive control scheme is developed based on the nominal controller structure, for the case of uncertain actuator failure. In Section VI, we study the generalize model of the cooperative robotic system with the adaptive actuator failure compensation scheme. Finally in Section VII, the simulation results of the algorithm are presented to confirm the effectiveness of our design.

\subsection{Problem Formulation}

The goal of this study is to develop an adaptive actuator failure compensation control scheme to ensure closed-loop stability and asymptotic output tracking of a general class of cooperative robotic systems in the presence of uncertain actuator failures. In this section, the control problem is formulated, and the associated technical issues are addressed based on a general cooperative robotic system model and a benchmark manipulator system.

\subsubsection{Cooperative Manipulator Systems}

Consider robotic systems that use multiple manipulators to fulfill a common task, such as to control a platform. The general dynamic model of such cooperative manipulator 
systems can be described as

$$
D_{c}\left(x_{o}\right) \ddot{x}_{o}+C_{c}\left(x_{o}, \dot{x}_{o}\right) \dot{x}_{o}+G_{c}\left(x_{o}\right)=E^{T}\left(x_{o}\right) \tau,
$$

where $x_{o}$ is the position and orientation of the platform, the combined inertia matrix of the object and manipulators $D_{c}\left(x_{o}\right)$ is assumed to be a bounded and positive definite matrix, $C_{c}\left(x_{o}, \dot{x}_{o}\right)$ is the Coriolis and centrifugal term, $G_{c}\left(x_{o}\right)$ is the gravity term,

$E$ is a transformation matrix which will be discussed later on, and $\tau=\left[\tau_{1}^{T}, \ldots, \tau_{p}^{T}\right]^{T}$, $\tau_{i}$ is the generalized torque vector of each manipulator, and $p$ is the number of manipulators in the system.

For the benchmark system model (to be described next) used in this study, we consider a system with three actuators. Since the system only needs two degrees of freedom, it is possible to develop an adaptive actuator failure compensation scheme for the system when one actuator in the system may fail. However, if two actuators in the system fail, it will be impossible to control the system to achieve a two-degree of freedom motion, with only one remaining actuator.

This concept is also applied to a system with higher degrees of freedom. For a system with $N$ degrees of freedom, we need at least $N$ remaining actuators to work in order to develop an adaptive actuator failure compensation scheme.

\subsubsection{Actuator Failure Model}

In this study, we will use the following actuator failure model.

Let $u(t)=\tau(t) \in R^{m}$ be the system input vector. When an actuator failure occurs, the control input component $u_{j}(t)$ associated with the failing actuator $j$ may 
become an arbitrary signal which can be expressed as

$$
u_{j}(t)=\bar{u}_{j}(t), t \geq t_{j}, j \in\{1,2, \ldots, m\} .
$$

Although we do not know the value of the control input when the failure occurs, we can model the failure based on some knowledge of the structure of the actuator failing signal as

$$
\bar{u}_{j}(t)=\bar{u}_{j 0}+\sum_{i=1}^{n_{j}} \bar{u}_{i j} f_{a i j}(t), t \geq t_{j},
$$

where $n_{j}$ is the number of components in the failing signal, $f_{a i j}$ are known functions corresponding to each component of the actuator, and $j, t_{j}, \bar{u}_{i j}$ are unknown failure index, failure time, and failure value of each component of the actuator.

\subsubsection{Control Objective}

In this study, we will develop an adaptive actuator failure compensation control framework for cooperative manipulator systems in the presence uncertain actuator failures. The objective of the adaptive failure compensation control scheme is to guarantee closed-loop system stability and output tracking, without the knowledge of the actuator failures.

The adaptive control scheme is applicable to the cooperative manipulator robotic system model of the form

$$
\begin{aligned}
& \dot{x}_{1}=x_{2} \\
& \dot{x}_{2}=g_{1}\left(x_{1}, x_{2}\right)+g_{2}\left(x_{1}, x_{2}\right) u(t)
\end{aligned}
$$

where $x_{1}=x_{o} \in R^{n}$ and $x_{2}=\dot{x}_{o} \in R^{n}$, are the states of the system, $y=x_{1} \in R^{n}$ 
is the output of the system, $g_{1} \in R^{n}, g_{2} \in R^{n x m}$ are known matrices based on the dynamic system, $n$ is the degree of freedom, $m$ is the number of the actuators, and $u \in R^{m}$ is the system input. Due to possible actuator failures, an applied feedback control signal $v(t)=\left[v_{1}, v_{2}, \ldots, v_{m}\right]^{T} \in R^{m}$ may not reach the system, as $u_{j}(t)$ is not equal to $v_{j}(t)$ if $u_{j}(t)=\bar{u}_{j}(t)$. More precisely, in the presence of actuator failures, the signal $u(t)$ is

$$
u(t)=(I-\sigma(t)) v(t)+\sigma(t) \bar{u}
$$

where $\sigma(t)=\operatorname{diag}\left\{\sigma_{1}, \sigma_{2}, \sigma_{3}\right\}$ is the actuator failure pattern matrix such that $\sigma_{i}(t)=1$ if the $i$ actuator fails, that is, if $u_{j}(t)=\bar{u}_{j}(t)$, and $\sigma_{i}(t)=0$ otherwise, and $\bar{u}(t)=$ $\left[\bar{u}_{1}, \ldots, \bar{u}_{m}\right]^{T}$.

The control objective is to design a feedback control signal $v(t)$ is such that $\lim _{t \rightarrow \infty}\left(g_{2}\left(x_{1}\right) u(t)-\omega_{d}(t)\right)=0$, despite the uncertain actuator failures $u(t)=(I-$ $\sigma(t)) v(t)+\sigma(t) \bar{u}$, where $\omega_{d}$ is a desirable control signal for $\omega$ to control the system model: $\dot{x}_{1}=x_{2}, \dot{x}_{2}=g_{1}\left(x_{1}, x_{2}\right)+\omega$, to make all signals in the closed-loop system are bounded and the output $y(t)=x_{1}(t)$ asymptotically tracks a given reference signal $y_{m}(t)$. When this objective is met, the real system $\dot{x}_{1}=x_{2}, \dot{x}_{2}=g_{1}\left(x_{1}, x_{2}\right)+g_{2}\left(x_{1}\right) u(t)$ will be ensured to have the desired signal boundedness and tracking properties, and this will be achieved through control adaptation.

The adaptive actuator failure compensation scheme is designed to compensate for actuator failure in the system without the knowledge of the failing actuator $j$, time that actuator failure occurs $t_{j}$, or value of the output of the failing actuator $\bar{u}_{j}(t)$, so that the system will remain stable and the output $y(t)$ asymptotically tracks the reference signal $y_{m}(t)$ even when actuator failures occur. 


\subsubsection{A Benchmark Cooperative Manipulator System}

In order to develop an adaptive control scheme for a general cooperative robotic system, we first consider a two-dimensional cooperative manipulator system as a benchmark system in our study. The system contains two manipulators, which are attached to each side of a rigid platform as shown in Figure 4.1. We will design a controller that can guarantee asymptotic tracking of both height $h(t)$ and angle $\theta(t)$ of the system. The adaptive control scheme for this sample robotic system can be extended to general cooperative robotic systems such as a Hexapod system or a humanoid robot, which will be discussed later on.

In many systems such as a humanoid robot, adding another leg to the robot for redundancy is not possible without the loss of functionality of the system. In our robotic system model, the redundancy of the system will came from an additional joint in a robotic manipulator. The system uses three actuators $q_{1}, q_{2}, q_{3}$ to support a rigid platform that links actuator $q_{1}$ and $q_{3}$ together. The actuator $q_{2}$ is added to increase redundancy in the system to compensate for possible actuator failure that could occur on the left side of the platform. In this study, we consider three cases of actuator failure patterns:

- Case 1: no actuator failure occurs, that is, $\sigma(t)=\operatorname{diag}\{0,0,0\}$, or

- Case 2: the actuator $q_{1}$ fails, $\sigma(t)=\operatorname{diag}\{1,0,0\}$, or

- Case 3: the actuator $q_{2}$ fails, $\sigma(t)=\operatorname{diag}\{0,1,0\}$.

With the benchmark model, we can derive the dynamic model of the system, and then we will develop the control scheme based on the dynamic model. The development of the adaptive actuator failure compensation scheme can be divided into three parts. First, we will find a feedback control signal $\omega_{d}$ from a backstepping 


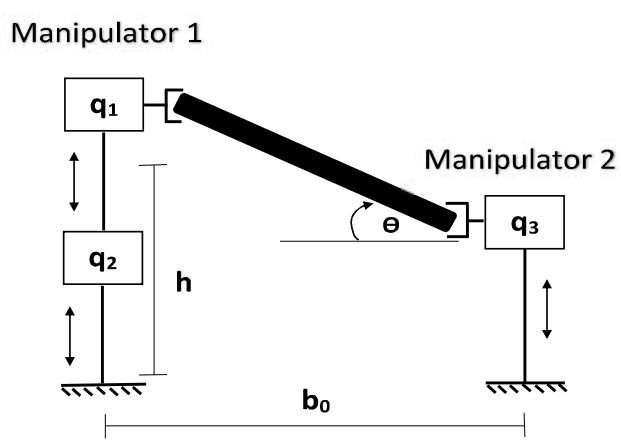

Figure 4.1: The benchmark cooperative robotic system.

control design, which ensures the stability and tracking of the system in the absence of actuator failure. Then, the nominal controller for the system will be developed based on the knowledge of actuator failure. The nominal control will be designed such that the control signal matches the signal $\omega_{d}$, so that the output of the system follows the desired trajectory. Finally, the adaptive actuator failure compensation scheme is developed based on the nominal controller structure, using parameter adaptation to handle the actuator failure uncertainties in the system.

\subsection{The Benchmark System Model}

The dynamic model of a redundant manipulator robotic system has been discussed in various books and published papers. There are several approaches that can be used to obtain a set of dynamic equations of the system, such as using Newton's second law of motion, or the Euler-Lagrange equation. Newton's second law of motion is a wellknown method of analyzing movement of an object. However, in order to calculate the dynamic of an object, one needs to know every force that acts on the object. Robotic systems, which contain many joints and links, it is complicated to calculate constraint forces in the system. As a result, using the Euler-Lagrange equation to 
develop the dynamic equation is a preferable method.

In this study, the derivation of the dynamic model of the system is divided in three parts. First, we derive dynamic equations of the system using the Euler-Lagrange equation. After we obtain the dynamic equations, state parameters of the system are defined and calculated. Finally, the dynamic equation is put into the state space form, so that it can be used for the development of the adaptive actuator failure compensation scheme.

\section{Euler-Lagrange Equation}

In order to use the Euler-Lagrange equation, we need to determine the generalized coordinates of the system. There are two sets of independent coordinates that should be considered. We can choose the height $h(t)$ and angle $\theta(t)$ of the platform as the generalized coordinates of the system. Although in this way the outputs of the system will be directly related to each coordinate, the dynamic equations are more complicated because the force of each actuator will act on different directions to each coordinate. On the other hand, by selecting the position of each actuator $q_{1}, q_{2}, q_{3}$ as the generalized coordinates, we can reduce the complexity of the derivation; as a result, we will use this second set as the generalized coordinates.

Before we derive the dynamic equations of the system, we consider the relationship between the coordinates and the outputs of the system.

From Figure 4.1, we can write the height and the angle of the platform in terms of the position of each actuator as

$$
\begin{aligned}
& h\left(q_{1}, q_{2}, q_{3}\right)=\frac{q_{1}+q_{2}+q_{3}}{2} \\
& \theta\left(q_{1}, q_{2}, q_{3}\right)=\arctan \left(\frac{q_{1}+q_{2}-q_{3}}{b_{0}}\right),
\end{aligned}
$$


where the constant $b_{0}$ is the length of the base of the platform. The derivatives of (4.6) and (4.7) are

$$
\begin{aligned}
\dot{h}\left(q_{1}, q_{2}, q_{3}\right) & =\frac{1}{2}\left(\dot{q}_{1}+\dot{q}_{2}+\dot{q}_{3}\right) \\
\dot{\theta}\left(q_{1}, q_{2}, q_{3}\right) & =\frac{b_{0}\left(\dot{q}_{1}+\dot{q}_{2}-\dot{q}_{3}\right)}{b_{0}^{2}+\left(q_{1}+q_{2}-q_{3}\right)^{2}} .
\end{aligned}
$$

Based on the generalize coordinate $q_{i}$ for $i=1,2,3$, we consider Lagrange's equation

$$
\frac{d}{d t}\left(\frac{\partial L}{\partial \dot{q}_{i}}\right)-\frac{\partial L}{\partial q_{i}}=\tau_{i},
$$

where the Lagrangian $L=T-V, \mathrm{~T}$ is the kinetic energy, $\mathrm{V}$ is the potential energy, $q_{i}=\left[q_{1}, q_{2}, q_{3}\right]$ are the vectors of generalized coordinates, and $u_{i}$ is the torque of each actuator, which acts along each coordinate. If the dependency forces between $q_{1}, q_{2}$ are small, we can approximate that the generalized coordinates are independent from each other. Thus, the kinetic energy and the potential energy can be written as

$$
\begin{aligned}
T & =\frac{1}{2}\left(m_{1} \dot{q}_{1}^{2}+\left(m_{1}+m_{2}\right) \dot{q}_{2}^{2}+m_{3} \dot{q}_{3}^{2}\right)+\frac{1}{2} m_{p} \dot{h}^{2}+\frac{1}{2} I_{p} \dot{\theta}^{2} \\
V & =m_{1} g q_{1}+\left(m_{1}+m_{2}\right) g q_{2}+m_{3} g q_{3}+m_{p} g h,
\end{aligned}
$$

where $\mathrm{g}$ is the scalar value of the gravity, $m_{i}$ is the mass of each actuator, $m_{p}$ is the mass of the platform, and $I_{p}$ is the moment of inertia of the platform.

We calculate each term in the Lagrange's equation as

$$
\frac{\partial L}{\partial q_{i}}=-\left(m_{i} g+\frac{1}{2} m_{p} g\right) ; i=1,2,3 .
$$


For the term $\frac{d}{d t} \frac{\partial L}{\partial \dot{q}_{i}}$, we first derive $\frac{\partial L}{\partial \dot{q}_{i}}$ as

$$
\begin{aligned}
& \frac{\partial L}{\partial \dot{q}_{1}}=m_{1} \dot{q}_{1}+m_{p} \dot{h} \frac{d \dot{h}}{d \dot{q}_{1}}+I_{p} \dot{\theta} \frac{d \dot{\theta}}{d \dot{q}_{1}} \\
& \frac{\partial L}{\partial \dot{q}_{2}}=\left(m_{1}+m_{2}\right) \dot{q}_{2}+m_{p} \dot{h} \frac{d \dot{h}}{d \dot{q}_{2}}+I_{p} \dot{\theta} \frac{d \dot{\theta}}{d \dot{q}_{2}} \\
& \frac{\partial L}{\partial \dot{q}_{3}}=m_{3} \dot{q}_{3}+m_{p} \dot{h} \frac{d \dot{h}}{d \dot{q}_{3}}+I_{p} \dot{\theta} \frac{d \dot{\theta}}{d \dot{q}_{3}} .
\end{aligned}
$$

From equation (4.8) we have $\frac{d \dot{h}}{d \dot{q}_{i}}=\frac{1}{2}$, and

$$
m_{p} \dot{h} \frac{d \dot{h}}{d \dot{q}_{i}}=\frac{1}{4} m_{p}\left(\dot{q}_{1}+\dot{q}_{2}+\dot{q}_{3}\right) .
$$

Consider $\dot{\theta} \frac{d \dot{\theta}}{d \dot{q}_{i}}$ based on equation (4.9), we have

$$
\dot{\theta} \frac{d \dot{\theta}}{d \dot{q}_{i}}=\frac{\alpha_{i} b_{0}^{2}\left(\dot{q}_{1}+\dot{q}_{2}-\dot{q}_{3}\right)}{\left(b_{0}^{2}+\left(q_{1}+q_{2}-q_{3}\right)^{2}\right)^{2}}
$$

where the constant $\alpha_{i}=1$ for $i=1,2, \alpha_{i}=-1$ for $i=3$. We define $\bar{q}_{1}=q_{1}+q_{2}+q_{3}$, $\bar{q}_{2}=q_{1}+q_{2}-q_{3}$, and $\Psi_{1}, \Psi_{2, i}$ as follows

$$
\Psi_{1}=\frac{1}{4} m_{p} \dot{\bar{q}}_{1}, \quad \Psi_{2, i}=\frac{\alpha_{i} b_{0}^{2} I_{p} \dot{\bar{q}}_{2}}{\left(b_{0}^{2}+\bar{q}_{2}^{2}\right)^{2}}
$$

Then, the time derivative $\frac{d}{d t}\left(\frac{\partial L}{\partial \dot{q}_{i}}\right)$ can be written as

$$
\begin{aligned}
& \frac{d}{d t} \frac{\partial L}{\partial \dot{q}_{1}}=m_{1} \ddot{q}_{1}+\dot{\Psi}_{1}+\dot{\Psi}_{2,1} \\
& \frac{d}{d t} \frac{\partial L}{\partial \dot{q}_{2}}=\left(m_{1}+m_{2}\right) \ddot{q}_{2}+\dot{\Psi}_{1}+\dot{\Psi}_{2,2} \\
& \frac{d}{d t} \frac{\partial L}{\partial \dot{q}_{3}}=m_{3} \ddot{q}_{3}+\dot{\Psi}_{1}+\dot{\Psi}_{2,3},
\end{aligned}
$$


where $\dot{\Psi}_{1}, \dot{\Psi}_{1}$ are expressed as

$$
\dot{\Psi}_{1}=\frac{1}{4} m_{p} \ddot{\bar{q}}_{1}, \quad \dot{\Psi}_{2, i}=\alpha_{i} b_{0}^{2} I_{p}\left(\frac{\ddot{\bar{q}}_{2}}{\left(b_{0}^{2}+\bar{q}_{2}^{2}\right)^{2}}-\frac{4 \bar{q}_{2} \dot{\bar{q}}_{2}^{2}}{\left(b_{0}^{2}+\bar{q}_{2}^{2}\right)^{3}}\right) .
$$

Finally, the Lagrange's equation becomes

$$
\begin{aligned}
m_{1} \ddot{q}_{1}+\dot{\Psi}_{1}+\dot{\Psi}_{2,1}+m_{1} g+\frac{1}{2} m_{p} g & =\tau_{1} \\
\left(m_{1}+m_{2}\right)\left(\ddot{q}_{2}+g\right)+\dot{\Psi}_{1}+\dot{\Psi}_{2,2}+\frac{1}{2} m_{p} g & =\tau_{2} \\
m_{3} \ddot{q}_{3}+\dot{\Psi}_{1}+\dot{\Psi}_{2,3}+m_{3} g+\frac{1}{2} m_{p} g & =\tau_{3} .
\end{aligned}
$$

\section{Determination of Signals $\ddot{\bar{q}}_{1}$ and $\ddot{\bar{q}}_{2}$}

In order to put the dynamic equations in the state space form, we need to rewrite the dynamic equations in term of $\ddot{q}_{1}$ and $\ddot{\bar{q}}_{2}$. Rewrite equations (24)-(26) for each $q_{i}$ as

$$
\begin{aligned}
& \ddot{q}_{1}=\frac{\tau_{1}-\left(\dot{\Psi}_{1}+\dot{\Psi}_{2,1}+\left(m_{1} g+\frac{1}{2} m_{p} g\right)\right)}{m_{1}} \\
& \ddot{q}_{2}=\frac{\tau_{2}-\dot{\Psi}_{1}-\dot{\Psi}_{2,2}}{m_{1}+m_{2}}-\frac{\left(\left(m_{1}+m_{2}\right) g+\frac{1}{2} m_{p} g\right)}{m_{1}+m_{2}} \\
& \ddot{q}_{3}=\frac{\tau_{3}-\left(\dot{\Psi}_{1}+\dot{\Psi}_{2,3}+\left(m_{3} g+\frac{1}{2} m_{p} g\right)\right)}{m_{3}} .
\end{aligned}
$$

Define a parameter $\zeta_{1}=1+\frac{m_{p}}{4}\left(\frac{1}{m_{1}}+\frac{1}{m_{1}+m_{2}}+\frac{1}{m_{3}}\right)$. We can combine equation (27)-(29) to get $\ddot{\bar{q}}_{1}$ as

$$
\begin{aligned}
\ddot{\bar{q}}_{1}= & \frac{1}{\zeta_{1}}\left(\left(\frac{\tau_{1}}{m_{1}}+\frac{\tau_{2}}{m_{1}+m_{2}}+\frac{\tau_{3}}{m_{3}}\right)-\left(3 g+\frac{m_{p} g}{2}\right)\left(\frac{1}{m_{1}}+\frac{1}{m_{1}+m_{2}}+\frac{1}{m_{3}}\right)\right. \\
& \left.-\dot{\Psi}_{2,1}\left(\frac{1}{m_{1}}+\frac{1}{m_{1}+m_{2}}+\frac{1}{m_{3}}\right)\right) .
\end{aligned}
$$


With a parameter $\zeta_{2}=\left(1+\frac{b_{0}^{2} I_{p}}{\left(b_{0}^{2}+\bar{q}_{2}^{2}\right)^{2}}\left(\frac{1}{m_{1}}+\frac{1}{m_{1}+m_{2}}+\frac{1}{m_{3}}\right)\right)$, we also have $\ddot{\bar{q}}_{2}$ as

$$
\begin{aligned}
\ddot{\bar{q}}_{2}= & \frac{1}{\zeta_{2}}\left(\left(\frac{\tau_{1}}{m_{1}}+\frac{\tau_{2}}{m_{1}+m_{2}}-\frac{\tau_{3}}{m_{3}}\right)-\dot{\Psi}_{1}\left(\frac{1}{m_{1}}+\frac{1}{m_{1}+m_{2}}-\frac{1}{m_{3}}\right)\right. \\
& \left.-\left(g+\frac{m_{p} g}{2}\right)\left(\frac{1}{m_{1}}+\frac{1}{m_{1}+m_{2}}-\frac{1}{m_{3}}\right)-\frac{4 b_{0}^{2} I_{p}\left(\bar{q}_{2} \dot{q}_{2}^{2}\right)}{b_{0}^{2}+\bar{q}_{2}^{2}}\left(\frac{1}{m_{1}}+\frac{1}{m_{1}+m_{2}}+\frac{1}{m_{3}}\right)\right) .
\end{aligned}
$$

Let parameters $M_{a}=\left(\frac{1}{m_{1}}+\frac{1}{m_{1}+m_{2}}+\frac{1}{m_{3}}\right)$, and $M_{b}=\left(\frac{1}{m_{1}}+\frac{1}{m_{1}+m_{2}}-\frac{1}{m_{3}}\right)$. Since $\Psi_{1}, \Psi_{2,1}$ contain $\ddot{\bar{q}}_{1}, \ddot{\bar{q}}_{2}$, we rewrite equation (30), and (31) as

$$
\begin{gathered}
\ddot{\bar{q}}_{1}=\frac{1}{\zeta_{1}}\left(\left(\frac{\tau_{1}}{m_{1}}+\frac{\tau_{2}}{m_{1}+m_{2}}+\frac{\tau_{3}}{m_{3}}\right)-\left(3 g+\frac{m_{p} g}{2}+\frac{b_{0}^{2} I_{p} \ddot{\bar{q}}_{2}}{\left(b_{0}^{2}+\bar{q}_{2}^{2}\right)^{2}}-\frac{4 b_{0}^{2} I_{p} \bar{q}_{2} \dot{\bar{q}}_{2}^{2}}{\left(b_{0}^{2}+\bar{q}_{2}^{2}\right)^{3}}\right) M_{a}\right) \\
\ddot{\bar{q}}_{2}=\frac{1}{\zeta_{2}}\left(\left(\frac{\tau_{1}}{m_{1}}+\frac{\tau_{2}}{m_{1}+m_{2}}-\frac{\tau_{3}}{m_{3}}-\frac{1}{4} m_{p}\left(\ddot{\bar{q}}_{1}\right)\right)-\left(\left(g+\frac{m_{p} g}{2}\right)\right) M_{b}+\frac{4 b_{0}^{2} I_{p}\left(\bar{q}_{2} \dot{q}_{2}^{2}\right)}{b_{0}^{2}+\bar{q}_{2}^{2}} M_{a}\right) .
\end{gathered}
$$

With $\zeta_{3}=\frac{b_{0}^{2} I_{p}}{\left(b_{0}^{2}+\bar{q}_{2}^{2}\right)^{2}} M_{a}$, by substitution of $\ddot{\bar{q}}_{2}$ from (33), equation (32) becomes

$$
\begin{aligned}
\ddot{\bar{q}}_{1} & =\frac{1}{\zeta_{1}}\left[\left(\frac{\tau_{1}}{m_{1}}+\frac{\tau_{2}}{m_{1}+m_{2}}+\frac{\tau_{3}}{m_{3}}\right)-\frac{\zeta_{3}}{\zeta_{2}}\left(\frac{\tau_{1}}{m_{1}}+\frac{\tau_{2}}{m_{1}+m_{2}}-\frac{\tau_{3}}{m_{3}}\right)\right. \\
& \left.+\frac{\zeta_{3}}{\zeta_{2}}\left(\frac{1}{4} m_{p}\left(\ddot{\bar{q}}_{1}\right)+\left(g+\frac{m_{p} g}{2}\right)\right) M_{b}-\left(3 g+\frac{m_{p} g}{2}\right)-\left(\frac{\zeta_{3}}{\zeta_{2}} \frac{4 b_{0}^{2} I_{p}\left(\bar{q}_{2} \dot{q}_{2}^{2}\right)}{b_{0}^{2}+\bar{q}_{2}^{2}}-\frac{4 b_{0}^{2} I_{p} \bar{q}_{2} \dot{\bar{q}}_{2}^{2}}{\left(b_{0}^{2}+\bar{q}_{2}^{2}\right)^{3}}\right) M_{a}\right] .
\end{aligned}
$$


With $\zeta_{4}=1+\frac{m_{p} \zeta_{3}}{4 \zeta_{1} \zeta_{2}} M_{b}$, we have

$$
\begin{aligned}
\ddot{\bar{q}}_{1}= & \frac{1}{\zeta_{4} \zeta_{1}}\left[\left(\frac{\tau_{1}}{m_{1}}+\frac{\tau_{2}}{m_{1}+m_{2}}+\frac{\tau_{3}}{m_{3}}\right)-\frac{\zeta_{3}}{\zeta_{2}}\left(\frac{\tau_{1}}{m_{1}}+\frac{\tau_{2}}{m_{1}+m_{2}}-\frac{\tau_{3}}{m_{3}}\right)\right. \\
& \left.+\frac{\zeta_{3}}{\zeta_{2}}\left(g+\frac{m_{p} g}{2}\right) M_{b}-\left(3 g+\frac{m_{p} g}{2}\right) M_{a}-\left(\frac{\zeta_{3}}{\zeta_{2}} \frac{4 b_{0}^{2} I_{p}\left(\bar{q}_{2} \dot{\bar{q}}_{2}^{2}\right)}{b_{0}^{2}+\bar{q}_{2}^{2}}-\frac{4 b_{0}^{2} I_{p} \bar{q}_{2} \dot{\bar{q}}_{2}^{2}}{\left(b_{0}^{2}+\bar{q}_{2}^{2}\right)^{3}}\right) M_{a}\right] .
\end{aligned}
$$

We solve for $\ddot{\bar{q}}_{2}$ with a similar approach. With $\zeta_{5}=\frac{m_{p}}{4} M_{b}$, by substitution of $\ddot{\bar{q}}_{1}$ from (35), equation (33) becomes

$$
\begin{aligned}
\ddot{\bar{q}}_{2}= & \frac{1}{\zeta_{2}}\left(\frac{\tau_{1}}{m_{1}}+\frac{\tau_{2}}{m_{1}+m_{2}}-\frac{\tau_{3}}{m_{3}}\right)-\frac{1}{\zeta_{2}}\left(\left(g+\frac{m_{p} g}{2}\right) M_{b}+\frac{4 b_{0}^{2} I_{p}\left(\bar{q}_{2} \dot{\bar{q}}_{2}^{2}\right)}{b_{0}^{2}+\bar{q}_{2}^{2}} M_{a}\right) \\
& -\frac{\zeta_{5}}{\zeta_{4} \zeta_{1} \zeta_{2}}\left[\left(\frac{\tau_{1}}{m_{1}}+\frac{\tau_{2}}{m_{1}+m_{2}}+\frac{\tau_{3}}{m_{3}}\right)+\frac{\zeta_{3}}{\zeta_{2}}\left(\frac{\tau_{1}}{m_{1}}+\frac{\tau_{2}}{m_{1}+m_{2}}-\frac{\tau_{3}}{m_{3}}\right)\right. \\
& \left.+\frac{\zeta_{3}}{\zeta_{2}}\left(g+\frac{m_{p} g}{2}\right) M_{b}+\left(3 g+\frac{m_{p} g}{2}\right) M_{a}+\frac{\zeta_{3}}{\zeta_{2}}\left(\frac{4 b_{0}^{2} I_{p}\left(\bar{q}_{2} \dot{\bar{q}}_{2}^{2}\right)}{b_{0}^{2}+\bar{q}^{2}}-\frac{4 b_{0}^{2} I_{p} \bar{q}_{2} \dot{\bar{q}}_{2}^{2}}{\left(b_{0}^{2}+\bar{q}_{2}^{2}\right)^{3}}\right) M_{a}\right] .
\end{aligned}
$$

\section{Dynamic Model in the State Space Form}

The dynamic equations can be written in the state space form. We define $x_{1}=$ $\left[x_{11}, x_{12}\right]^{T}=[h, \theta]^{T}$, and $x_{2}=\left[x_{21}, x_{22}\right]^{T}=\left[\dot{\bar{q}}_{1}, \dot{\bar{q}}_{2}\right] .^{T}$ With $\bar{q}_{1}=2 h, \bar{q}_{2}=b_{0} \tan \theta$, we have the system in state space form as

$$
\begin{aligned}
& \dot{x}_{1}=\left[\begin{array}{ll}
\frac{1}{2} \dot{\bar{q}}_{1} & \frac{1}{b_{0}\left(1+\theta^{2}\right)} \dot{\bar{q}}_{2}
\end{array}\right]^{T} \\
& \dot{x}_{2}=\left[\begin{array}{ll}
\left(\ddot{q}_{1}+\ddot{q}_{2}+\ddot{q}_{3}\right) & \left(\ddot{q}_{1}+\ddot{q}_{2}-\ddot{q}_{3}\right)
\end{array}\right]^{T} .
\end{aligned}
$$

Let $u=\left[\tau_{1}, \tau_{2}, \tau_{3}\right]$ be the system input, and $y=[h, \theta]^{T}$ be the system output. The 
state equation can be written as

$$
\begin{aligned}
& \dot{x}_{1}=f\left(x_{1}\right) x_{2} \\
& \dot{x}_{2}=g_{1}\left(x_{1}, x_{2}\right)+g_{2}\left(x_{1}, x_{2}\right) u \\
& y=x_{1}
\end{aligned}
$$

where the matrix $f\left(x_{1}\right)$ is expressed as

$$
f\left(x_{1}\right)=\left[\begin{array}{cc}
f_{11}\left(x_{1}\right) & 0 \\
0 & f_{22}\left(x_{1}\right)
\end{array}\right]
$$

We have

$$
f_{11}\left(x_{1}\right)=\frac{1}{2}, \quad f_{22}\left(x_{1}\right)=\frac{1}{b_{0}\left(1+x_{12}^{2}\right)}
$$

The matrix $g_{1}\left(x_{1}, x_{2}\right), g_{2}\left(x_{1}, x_{2}\right)$ are known matrices, which can be calculated from the Lagrange's equation. The matrix $g_{1}\left(x_{1}, x_{2}\right)=\left[g_{11}\left(x_{1}, x_{2}\right), g_{12}\left(x_{1}, x_{2}\right)\right] \cdot{ }^{T}$ Consider equations (4.35) and (4.36), we obtain

$$
\begin{aligned}
g_{11}= & \frac{1}{\zeta_{4} \zeta_{1}}\left[\frac{\zeta_{3}}{\zeta_{2}}\left(g+\frac{m_{p} g}{2}\right) M_{b}-\left(\left(3 g+\frac{m_{p} g}{2}\right)+\frac{\zeta_{3}}{\zeta_{2}} \frac{4 b_{0}^{2} I_{p}\left(\bar{q}_{2} x_{22}^{2}\right)}{b_{0}^{2}+\bar{q}_{2}^{2}}-\frac{4 b_{0}^{2} I_{p} \bar{q}_{2} x_{22}^{2}}{\left.\left.\left(b_{0}^{2}+\bar{q}_{2}^{2}\right)^{3}\right) M_{a}\right]}\right.\right. \\
g_{12}= & \frac{1}{\zeta_{2}}\left[-\left(g+\frac{m_{p} g}{2}\right) M_{b}-\frac{4 b_{0}^{2} I_{p}\left(\bar{q}_{2} x_{22}^{2}\right)}{b_{0}^{2}+\bar{q}_{2}^{2}} M_{a}\right]-\frac{\zeta_{5}}{\zeta_{4} \zeta_{1} \zeta_{2}}\left[\frac{\zeta_{3}}{\zeta_{2}}\left(g+\frac{m_{p} g}{2}\right) M_{b}+\left(\left(3 g+\frac{m_{p} g}{2}\right)\right.\right. \\
& \left.\left.+\frac{\zeta_{3}}{\zeta_{2}} \frac{4 b_{0}^{2} I_{p}\left(\bar{q}_{2} x_{22}^{2}\right)}{b_{0}^{2}+\bar{q}_{2}^{2}}-\frac{4 b_{0}^{2} I_{p} \bar{q}_{2} x_{22}^{2}}{\left(b_{0}^{2}+\bar{q}_{2}^{2}\right)^{3}}\right) M_{a}\right] .
\end{aligned}
$$

Define the matrix $g_{2}\left(x_{1}, x_{2}\right)$ as

$$
g_{2}\left(x_{1}, x_{2}\right)=\left[\begin{array}{lll}
g_{211} & g_{212} & g_{213} \\
g_{221} & g_{222} & g_{223}
\end{array}\right]
$$


According to the equations (35) and (36), we have each component of the matrix as

$$
\begin{aligned}
& g_{211}=\frac{1}{\zeta_{4} \zeta_{1}}\left[\left(1-\frac{\zeta_{3}}{\zeta_{2}}\right)\left(\frac{1}{m_{1}}\right)\right], \quad g_{212}=\frac{1}{\zeta_{4} \zeta_{1}}\left[\left(1-\frac{\zeta_{3}}{\zeta_{2}}\right)\left(\frac{1}{m_{1}+m_{2}}\right)\right], \\
& g_{213}=\frac{1}{\zeta_{4} \zeta_{1}}\left[\left(1+\frac{\zeta_{3}}{\zeta_{2}}\right)\left(\frac{1}{m_{3}}\right)\right], \quad g_{221}=\left(\frac{1}{\zeta_{2}}-\frac{\zeta_{5}}{\zeta_{4} \zeta_{1} \zeta_{2}}\left(1-\frac{\zeta_{3}}{\zeta_{2}}\right)\right)\left(\frac{1}{m_{1}}\right), \\
& g_{222}=\left(\frac{1}{\zeta_{2}}-\frac{\zeta_{5}}{\zeta_{4} \zeta_{1} \zeta_{2}}\left(1-\frac{\zeta_{3}}{\zeta_{2}}\right)\right)\left(\frac{1}{m_{1}+m_{2}}\right), \quad g_{223}=\left(-\frac{1}{\zeta_{2}}-\frac{\zeta_{5}}{\zeta_{4} \zeta_{1} \zeta_{2}}\left(1+\frac{\zeta_{3}}{\zeta_{2}}\right)\right)\left(\frac{1}{m_{3}}\right) .
\end{aligned}
$$

In this way, we have derived the state space form of the dynamic model of the cooperative manipulator robotic system with actuator redundancy, which can be used to design an adaptive actuator failure compensation scheme. 


\subsection{Nominal Control Design}

In this section we develop a nominal controller for the system, assuming the knowledge of the actuator failures. Such a controller structure can be used to design an adaptive actuator failure compensation scheme for the system with uncertain actuator failures. From the dynamic model derived in Section III, we have

$$
\begin{aligned}
& \dot{x}_{1}=f\left(x_{1}\right) x_{2} \\
& \dot{x}_{2}=g_{1}\left(x_{1}, x_{2}\right)+g_{2}\left(x_{1}, x_{2}\right) u
\end{aligned}
$$

where $y=[h, \theta]^{T}$ is the system output, and $f\left(x_{1}\right), g_{1}\left(x_{1}, x_{2}\right), g_{2}\left(x_{1}, x_{2}\right)$ are known matrices.

Our control objective is to control the height $h(t)$ and angle $\theta(t)$ of the platform, to make the system output $y(t)$ tracks a desirable trajectory $y_{m}(t)$ asymptotically. First, we generate a desirable feedback control signal $\omega_{d}=g_{2}\left(x_{1}, x_{2}\right) u$ from a backstepping control design method, and then we develop a nominal failure compensation controller based on the feedback control signal, $w_{d}$, and the failure of the actuator signals.

\subsubsection{Backstepping Control Design}

We will first choose a control signal $\omega_{d}$ such that it can guarantee the closed-loop signal boundedness and asymptotic output tracking of the system in the absence of actuator failures [17].

Define $z_{1}=x_{1}-y_{m}, z_{2}=x_{2}-\beta$, where $\beta$ is a design function to be determind. 
Step 1: From the state equation of the system, we can write $\dot{z}_{1}$ as

$$
\dot{z}_{1}=\dot{x}_{1}-\dot{y}_{m}=f\left(x_{1}\right)\left(z_{2}+\beta\right)-\dot{y}_{m} .
$$

In order to choose $\beta$ to stablize the system, we consider a Lyapunov candidate function and its derivative as

$$
\begin{aligned}
& V_{1}=\frac{1}{2} z_{1}^{T} z_{1} \\
& \dot{V}_{1}=z_{1}\left(f\left(x_{1}\right) z_{2}+f\left(x_{1}\right) \beta-\dot{y}_{m}\right) .
\end{aligned}
$$

With the design function

$$
\beta=f\left(x_{1}\right)^{-1}\left(-c_{1} z_{1}+\dot{y}_{m}\right),
$$

where $c_{1}$ is a positive constant, the derivative of the Lyapunov function becomes

$$
\dot{V}_{1}=-c_{1} z_{1}^{T} z_{1}+f\left(x_{1}\right) z_{1} z_{2}
$$

We see that the term $-c_{1} z_{1}^{T} z_{1}$ is always negative.

Step 2: Since $z_{2} \neq 0$, we continue using backstepping by considering

$$
\dot{z}_{2}=\dot{x}_{2}-\dot{\beta}=g_{1}\left(x_{1}, x_{2}\right)+g_{2}\left(x_{1}, x_{2}\right) u-\dot{\beta},
$$

and the second Lyapunov function

$$
V_{2}=V_{1}+\frac{1}{2} z_{2}^{T} z_{2}
$$


The time-derivative of $V_{2}$ is

$$
\dot{V}_{2}=\dot{V}_{1}+z_{2} \dot{z}_{2}=-c_{1} z_{1}^{T} z_{1}+z_{2}^{T}\left(f\left(x_{1}\right) z_{1}+g_{1}\left(x_{1}, x_{2}\right)+g_{2}\left(x_{1}, x_{2}\right) u-\dot{\beta}\right)
$$

If the signal $\omega_{d}$ can be chosen as

$$
\begin{aligned}
\omega_{d} & =g_{2}\left(x_{1}, x_{2}\right) u \\
& =-g_{1}\left(x_{1}, x_{2}\right)-c_{2} z_{2}-f\left(x_{1}\right) z_{1}+\dot{\beta} .
\end{aligned}
$$

Equation (29) becomes

$$
\dot{V}_{2}=-c_{1} z_{1}^{T} z_{1}-c_{2} z_{2}^{T} z_{2}
$$

With a positive constant $c_{2}, \dot{V}_{2}$ is negative definite. As a result, the control signal $\omega_{d}$ can guarantee the closed-loop signal boundedness and asymptotic output tracking of the system: $\lim _{t \rightarrow \infty}\left(y(t)-y_{m}(t)\right)=0$.

\subsubsection{Nominal Actuator Failure Controller Structure}

In this subsection, we develop a nominal controller for the system, with knowledge of actuator failures. The nominal control law will guarantee that the control input signal matches the desirable signal $\omega_{d}$ when actuator failures occur.

Since the controller needs to handle several cases of actuator failure, we select a nominal controller structure as a combination of the control law for each actuator failure case. We first design three individual control laws for three actuator failure case $[18]$. 


\section{Design for the no failure case}

In the case of no actuator failure in the system, we have control signal $u=v^{*}$. We need to design $v(t)$ such that $\omega_{d}=g_{2}\left(x_{1}, x_{2}\right) v^{*}$. We consider

$$
v^{*}(t)=v_{(1)}^{*}(t)=h_{a}\left(x_{1}, x_{2}\right) v_{a(1)}^{*}(t)
$$

where $h_{a}\left(x_{1}, x_{2}\right) \in R^{3 \times 3}$, and $v_{a(1)}^{*}(t) \in R^{3 \times 1}$ are such that

$$
g_{2}\left(x_{1}, x_{2}\right) h_{a}\left(x_{1}, x_{2}\right) v_{a(1)}^{*}=\omega_{d}
$$

Notice that in case of no actuator failure, we have some flexibilities when choosing the matrix $h_{a}\left(x_{1}, x_{2}\right)$. We can choose the matrix such that the system is optimized subject to some desirable constrains. With chosen $h_{a}\left(x_{1}, x_{2}\right)$, the intermediate control signal can be written as

$$
v_{a(1)}^{*}(t)=K_{a}\left(x_{1}, x_{2}\right) \omega_{d},
$$

One way to design the controller to have a unique solution is to use only two actuators to control the system. By turning off actuator $u_{1}$, we can design the controller by choosing

$$
\begin{aligned}
v^{*}(t) & =v_{(1)}^{*}(t)=\left[\begin{array}{ll}
0 & v_{b(1)}^{* T}(t)
\end{array}\right]^{T} \\
v_{b(1)}^{*}(t) & =h_{1}\left(x_{1}, x_{2}\right) v_{0(1)}^{*}(t) .
\end{aligned}
$$

With $g_{2}=\left[g_{21}, g_{22}, g_{23}\right]=\left[g_{21}, g_{2(2)}\right]$, we can choose a new matrix $h_{1}\left(x_{1}, x_{2}\right) \in R^{2 \times 2}$ 
such that $g_{2(2)}\left(x_{1}, x_{2}\right) h_{1}\left(x_{1}, x_{2}\right)$ is invertible. Thus, $v_{0(1)}^{*}(t) \in R^{2 \times 1}$ can be chosen as

$$
v_{0(1)}^{*}(t)=K_{1}\left(x_{1}, x_{2}\right) \omega_{d}
$$

where the matrix $K_{1}\left(x_{1}, x_{2}\right) \in R^{2 \times 2}$ is expressed as

$$
K_{1}\left(x_{1}, x_{2}\right)=\left(g_{2(2)}\left(x_{1}, x_{2}\right) h_{1}\left(x_{1}, x_{2}\right)\right)^{-1}
$$

As a result, we have the nominal control signal $v_{(1)}^{*}(t)$ that can be used to control the system with no actuator failure.

\section{Design for the $u_{1}$ failure case}

In case of actuator $u_{1}$ failure, we have the signals $u_{1}=\bar{u}_{1}, u_{2}=v_{2}^{*}$, and $u_{3}=v_{3}^{*}$. The nominal control signal can be chosen as

$$
v^{*}(t)=v_{(2)}^{*}(t)=\left[\begin{array}{ll}
0 & v_{a(2)}^{* T}(t)
\end{array}\right]^{T}
$$

With $g_{2}=\left[g_{21}, g_{22}, g_{23}\right]=\left[g_{21}, g_{2(2)}\right]$, we have

$$
v_{a(2)}^{*}(t)=h_{2}\left(x_{1}, x_{2}\right) v_{0(2)}^{*}(t),
$$

where the matrix $h_{2}\left(x_{1}, x_{2}\right) \in R^{2 \times 2}$ and the signal $v_{0(2)}^{*}(t) \in R^{2 \times 1}$ can be chosen to satisfy

$$
g_{21}\left(x_{1}, x_{2}\right) \bar{u}_{1}+g_{2(2)} h_{2}\left(x_{1}, x_{2}\right) v_{0(2)}^{*}(t)=\omega_{d}
$$


The signal $v_{0(2)}^{*}(t)$ can be written as

$$
v_{0(2)}^{*}(t)=K_{21}\left(x_{1}, x_{2}\right) \omega_{d}+K_{22}\left(x_{1}, x_{2}\right) \bar{u}_{1},
$$

where $K_{21} \in R^{2 \times 2}, K_{22} \in R^{2 \times 1}$ are expressed as

$$
\begin{aligned}
& K_{21}=\left(g_{2(2)} h_{2}\left(x_{1}, x_{2}\right)\right)^{-1} \\
& K_{22}=-\left(g_{2(2)} h_{2}\left(x_{1}, x_{2}\right)\right)^{-1} g_{21}\left(x_{1}, x_{2}\right)
\end{aligned}
$$

We can see that with this design, the nominal control signal $v_{(2)}^{*}(t)$ ensures $\omega_{d}=$ $g_{2}\left(x_{1}, x_{2}\right) v_{(2)}^{*}$ for the actuator $u_{1}$ failure case.

\section{Design for the $u_{2}$ failure case}

Similarly, in case of actuator $u_{2}$ failure. The nominal control signal can be designed as

$$
v^{*}(t)=v_{(3)}^{*}(t)=\left[v_{1(3)}^{*}(t), 0, v_{3(3)}^{*}(t)\right]^{T}
$$

Defining $v_{a(3)}^{*}(t) \in R^{2 \times 1}$ as

$$
v_{a(3)}^{*}(t)=\left[v_{1(3)}^{*}(t), v_{3(3)}^{*}(t)\right]^{T} .
$$

We design the control law from

$$
v_{a(3)}^{*}(t)=h_{3}\left(x_{1}, x_{2}\right) v_{0(3)}^{*}(t) .
$$


With $g_{2(1)}=\left[g_{21}, g_{23}\right]$, we can choose $h_{3}\left(x_{1}, x_{2}\right) \in R^{2 \times 2}$, and $v_{0(3)}^{*}(t) \in R^{2 \times 1}$ to satisfy

$$
g_{2(1)}\left(x_{1}, x_{2}\right) h_{3}\left(x_{1}, x_{2}\right) v_{0(3)}^{*}(t)+g_{22} \bar{u}_{2}=\omega_{d}
$$

The signal $v_{0(3)}^{*}(t)$ can be written as

$$
v_{0(3)}^{*}(t)=K_{31}\left(x_{1}, x_{2}\right) \bar{u}_{2}+K_{32}\left(x_{1}, x_{2}\right) \omega_{d}
$$

where $K_{31} \in R^{2 \times 1}, K_{32} \in R^{2 \times 2}$ are expressed as

$$
\begin{aligned}
& K_{31}=\left(g_{2(1)}\left(x_{1}, x_{2}\right) h_{3}\left(x_{1}, x_{2}\right)\right)^{-1} g_{22}\left(x_{1}, x_{2}\right) \\
& K_{32}=-\left(g_{2(1)}\left(x_{1}, x_{2}\right) h_{3}\left(x_{1}, x_{2}\right)\right)^{-1} .
\end{aligned}
$$

We can see that the nominal control signal $v_{(3)}^{*}(t)$ ensures $\omega_{d}=g_{2}\left(x_{1}, x_{2}\right) v_{(3)}^{*}$ for the actuator $u_{2}$ failure case.

\section{Composite control design}

With the control structure for all three cases, we can design a composite control law for the system as

$$
v^{*}(t)=\chi_{1}^{*} v_{(1)}^{*}(t)+\chi_{2}^{*} v_{(2)}^{*}(t)+\chi_{3}^{*} v_{(3)}^{*}(t)
$$

where $\chi_{1}^{*}, \chi_{2}^{*}, \chi_{3}^{*}$ are the indicator functions of the actuator failure: $\chi_{1}^{*}=1$ when there is no actuator failure, $\chi_{2}^{*}=1$ for $u_{1}$ actuator failure, and $\chi_{3}^{*}=1$ for $u_{2}$ actuator failure. The indicator functions $\chi_{1}^{*}, \chi_{2}^{*}, \chi_{3}^{*}=0$ for its noncorresponding cases, e.g., $\chi_{1}^{*}=0$ when actuator $u_{1}$ fails. Signal $v_{(1)}^{*}(t)$ is the nominal control signal in case of no actuator failure in the system, $v_{(2)}^{*}(t)$ is the nominal control signal for the actuator 
$u_{1}$ failure case, and $v_{(3)}^{*}(t)$ is the nominal control signal for actuator $u_{2}$ failure case.

Because of the indicator functions and the nominal control signals, the composite design ensures $\omega_{d}=g_{2}\left(x_{1}, x_{2}\right) v^{*}(t)$ for every case of actuator failure. The composite control design can control the system with an actuator failure when the information of actuator failure is known.

\subsection{Adaptive Actuator Failure Compensation}

The nominal control design, which is developed in Section 4.4, needs information of the actuator failures. In this section, we develop an adaptive actuator failure compensation scheme, which can achieve the control objective in the presence of uncertain actuator failures without the knowledge of the failing actuator and its signal.

\subsubsection{Adaptive Controller Structure}

The adaptive control algorithm is developed based on the nominal controller structure. Since we do not know the values of $\chi_{1}^{*} v_{(1)}^{*}, \chi_{2}^{*} v_{(2)}^{*}$, and $\chi_{3}^{*} v_{(3)}^{*}$, we first design the adaptive controller structure as

$$
v(t)=v_{\chi(1)}(t)+v_{\chi(2)}(t)+v_{\chi(3)}(t)
$$

where $v_{\chi(1)}(t), v_{\chi(2)}(t), v_{\chi(3)}(t)$ are the estimates of $\chi_{1}^{*} v_{(1)}^{*}, \chi_{2}^{*} v_{(2)}^{*}, \chi_{3}^{*} v_{(3)}^{*}$ respectively.

In order to derive $v_{\chi(1)}(t)$, we restructure $\chi_{1}^{*} v_{(1)}^{*} \in R^{3 \times 1}$ from the nonimal controller 
(4.61) as

$$
\chi_{1}^{*} v_{(1)}^{*}=\chi_{1}^{*} h_{a} K_{a} \omega_{d}=\operatorname{diag}\left\{\chi_{11}^{*}, \chi_{12}^{*}, \chi_{13}^{*}\right\} h_{a} K_{a} \omega_{d}
$$

where $\chi_{11}^{*}=\chi_{12}^{*}=\chi_{13}^{*}=\chi_{1}^{*}$, that is, we will estimate the parameter $\chi_{1}^{*}$ three times for achieving a suitable parametrization. With the estimated parameter $\operatorname{diag}\left\{\chi_{11}, \chi_{12}, \chi_{13}\right\}$, we have the adaptive version of $\chi_{1}^{*} v_{(1)}^{*}$ as

$$
v_{\chi(1)}(t)=\operatorname{diag}\left\{\chi_{11}, \chi_{12}, \chi_{13}\right\} h_{a} K_{a} \omega_{d}
$$

With $v_{\chi(2)}(t)=\left[0, v_{\chi a(2)}(t)^{T}\right]^{T}$, we now derive $v_{\chi a(2)}(t)$ from its nominal version:

$$
\chi_{2}^{*} v_{a(2)}^{*}(t)=\chi_{2}^{*} h_{2} K_{21} \omega_{d}+\chi_{2}^{*} \bar{u}_{1} h_{2} K_{22} \in R^{2 \times 1}
$$

From the actuator failure model (4.3), the actuator failure $\bar{u}_{i}(t)$ can be expressed as

$$
\bar{u}_{i}(t)=\rho_{i}^{* T} f_{a i}(t)
$$

where $f_{a i}(t)=\left[1, f_{a i 1}(t), \ldots, f_{a i n_{i}}(t)\right]^{T}$ are known functions corresponding to the actuator failure components, $\rho_{i}^{*}=\left[\bar{u}_{i 0}, \bar{u}_{i 1}, \ldots, \bar{u}_{i n_{i}}\right]^{T}$ contains the paremeters of values associated with each actuator failure component, and $n_{i}$ is the number of actuator failure components.

Then, the estimate of $\chi_{2}^{*} v_{a(2)}^{*}(t)$ is chosen as

$$
v_{\chi a(2)}(t)=\operatorname{diag}\left\{\chi_{21}, \chi_{22}\right\} h_{2} K_{21} \omega_{d}+\left[\begin{array}{c}
\rho_{1(1)}^{T} f_{a 1}(t) \phi_{11} \\
\rho_{1(2)}^{T} f_{a 1}(t) \phi_{12},
\end{array}\right]
$$


where, similar to that in (4.83), $\operatorname{diag}\left\{\chi_{21}, \chi_{22}\right\}$ is the estimate of $\operatorname{diag}\left\{\chi_{21}^{*}, \chi_{22}^{*}\right\}$ with $\chi_{2}^{*}=\chi_{21}^{*}=\chi_{22}^{*}$. The terms $\chi_{21}^{*} \rho_{1}^{*}$ and $\chi_{22}^{*} \rho_{1}^{*}$ are estimated by $\rho_{1(1)}$ and $\rho_{1(2)}$, where $\phi_{1}=\left[\phi_{11}, \phi_{12}\right]^{T}=h_{2} K_{22}$.

In order to derive $v_{\chi(3)}$, we consider $v_{\chi(3)}=\left[v_{\chi(31)}, 0, v_{\chi(33)}\right]^{T}$. With $v_{\chi a(3)}=$ $\left[v_{\chi(31)}, v_{\chi(33)}\right]^{T}$, we consider

$$
\chi_{3}^{*} v_{a(3)}^{*}(t)=\chi_{3}^{*} h_{3} K_{31} \bar{u}_{2}+\chi_{3}^{*} h_{3} K_{32} \omega_{d} \in R^{2 \times 1} .
$$

Similar to the derivation of $v_{\chi a(2)}(t)$, we have

$$
v_{\chi a(3)}(t)=\operatorname{diag}\left\{\chi_{31}, \chi_{32}\right\} h_{3} K_{32} \omega_{d}+\left[\begin{array}{c}
\rho_{2(1)}^{T} f_{a 2}(t) \phi_{21} \\
\rho_{2(2)}^{T} f_{a 2}(t) \phi_{22},
\end{array}\right]
$$

where $\operatorname{diag}\left\{\chi_{31}, \chi_{32}\right\}$ is the estimate of $\operatorname{diag}\left\{\chi_{31}^{*}, \chi_{32}^{*}\right\}$ with $\chi_{3}^{*}=\chi_{31}^{*}=\chi_{32}^{*}$. The parameters $\rho_{2(1)}$ and $\rho_{2(2)}$ are the estimates of $\chi_{31}^{*} \rho_{2}^{*}$ and $\chi_{32}^{*} \rho_{2}^{*}$, where $\phi_{2}=\left[\phi_{21}, \phi_{22}\right]^{T}=$ $h_{3} K_{31}$.

The controller structure (4.80) can be written as

$$
v(t)=v_{\chi(1)}(t)+\left[0, v_{a \chi(2)}^{T}(t)\right]^{T}+\left[v_{\chi(31)}(t), 0, v_{\chi(33)}(t)\right]^{T} .
$$

With this parametrized controller structure, we can develop adaptive laws to update the parameters $\operatorname{diag}\left\{\chi_{11}, \chi_{12}, \chi_{13}\right\}, \operatorname{diag}\left\{\chi_{21}, \chi_{22}\right\}, \operatorname{diag}\left\{\chi_{31}, \chi_{32}\right\}, \rho_{1(1)}, \rho_{1(2)}, \rho_{2(1)}$, and $\rho_{2(2)}$, so that the system can achieve the control objective in the presence of uncertain actuator failure. 


\subsubsection{Error System}

In order to design an adaptive law for the system, we consider the system error caused by an actuator failure. With an uncertain actuator failure, the signal $u(t)$ can be written as

$$
u(t)=(I-\sigma(t)) v^{*}(t)+\sigma(t) \bar{u},
$$

where $\sigma(t)=\operatorname{diag}\left\{\sigma_{1}, \sigma_{2}, \sigma_{3}\right\}$ is the actuator failure pattern matrix such that $\sigma_{i}(t)=1$ if the $i$ actuator fails, and $\sigma_{i}(t)=0$ otherwise. With $g_{2}\left(x_{1}, x_{2}\right) u(t)=\omega_{d}$, we have

$$
g_{2}\left(x_{1}, x_{2}\right)\left((I-\sigma) v^{*}(t)+\sigma \bar{u}\right)=\omega_{d}
$$

For an adaptive updated control signal $v(t)$, where $g_{2}\left(x_{1}, x_{2}\right) v(t)=\omega$. Based on equations (4.89) and (4.90), we have an error system between $v(t)$ and $v^{*}(t)$ as

$$
g_{2}\left(x_{1}, x_{2}\right)(I-\sigma)\left(v-v^{*}\right)=\omega-\omega_{d} .
$$

We consider the backstepping design with a possible actuator failure. With $z_{1}=$ $x_{1}-y_{m}, z_{2}=x_{2}-\beta$, where $\beta$ is a design function from equation (4.53), we have

$$
\begin{aligned}
\dot{z}_{1} & =-c_{1} z_{1}+f\left(x_{1}\right) z_{2} \\
\dot{z}_{2} & =g_{1}\left(x_{1}, x_{2}\right)+g_{2}\left(x_{1}, x_{2}\right) u-\dot{\beta} \\
& =g_{1}\left(x_{1}, x_{2}\right)+\omega-\dot{\beta}+\left(\omega_{d}-\omega_{d}\right) \\
& =-f\left(x_{1}\right) z_{1}-c_{2} z_{2}+\omega-\omega_{d} .
\end{aligned}
$$

From equations (4.91) and (4.93), $\dot{z}_{2}$ becomes 


$$
\dot{z}_{2}=-f\left(x_{1}\right) z_{1}-c_{2} z_{2}+g_{2}\left(x_{1}, x_{2}\right)(I-\sigma)\left(v-v^{*}\right) .
$$

Define the error signal $\tilde{v}=v-v^{*}$, and the error of each estimated parameter as $\tilde{\chi}_{i j}=\chi_{i j}-\chi_{i}^{*}, \tilde{\rho}_{i(j)}=\rho_{i(j)}-\left(\chi_{i+1 j}^{*} \rho_{i}^{*}\right)$. From equations (4.80) and (4.91), the error signal $\tilde{v}$ can be written as

$$
\tilde{v}=\operatorname{diag}\left\{\tilde{\chi}_{11}, \tilde{\chi}_{12}, \tilde{\chi}_{13}\right\} h_{a} K_{a} \omega_{d}+\left[0, \tilde{v}_{\chi a(2)}^{T}(t)\right]^{T}+\left[\tilde{v}_{\chi(31)}(t), 0, \tilde{v}_{\chi(33)}(t)\right]^{T} .
$$

Based on equations (4.85) and (4.87) with $\tilde{v}_{\chi a(3)}(t)=\left[\tilde{v}_{\chi(31)}(t), \tilde{v}_{\chi(33)}(t)\right]^{T}$, we have

$$
\begin{aligned}
& \tilde{v}_{\chi a(2)}(t)=\operatorname{diag}\left\{\tilde{\chi}_{21}, \tilde{\chi}_{22}\right\} h_{2} K_{21}\left(x_{1}, x_{2}\right) \omega_{d}+\left[\begin{array}{c}
\tilde{\rho}_{1(1)}^{T} f_{a 1}(t) \phi_{11} \\
\tilde{\rho}_{1(2)}^{T} f_{a 1}(t) \phi_{12},
\end{array}\right] \\
& \tilde{v}_{\chi a(3)}(t)=\operatorname{diag}\left\{\tilde{\chi}_{31}, \tilde{\chi}_{32}\right\} h_{2} K_{32}\left(x_{1}, x_{2}\right) \omega_{d}+\left[\begin{array}{c}
\tilde{\rho}_{2(1)}^{T} f_{a 2}(t) \phi_{21} \\
\tilde{\rho}_{2(2)}^{T} f_{a 2}(t) \phi_{22},
\end{array}\right] .
\end{aligned}
$$

The effect of actuator failure to the nominal controller can be analyzed using the Lyapunov function

$$
V_{2}=\frac{1}{2}\left(z_{1}^{T} z_{1}+z_{2}^{T} z_{2}\right)
$$

Based on equation (4.92) and (4.94), the derivative of the Lyapunov function (4.97) becomes

$$
\dot{V}_{2}=-c_{1} z_{1}^{T} z_{1}-c_{2} z_{2}^{T} z_{2}+z_{2} g_{2}\left(x_{1}, x_{2}\right)(I-\sigma)(\tilde{v}) .
$$


With $\tilde{v}$ from equation (4.95), the derivative of the Lyapunov function is

$$
\begin{aligned}
\dot{V}_{2} & =-c_{1} z_{1}^{T} z_{1}-c_{2} z_{2}^{T} z_{2}+z_{2}^{T} g_{2}\left(x_{1}, x_{2}\right)(I-\sigma) \operatorname{diag}\left\{\tilde{\chi}_{11}, \tilde{\chi}_{12}, \tilde{\chi}_{13}\right\} h_{a} K_{a} \omega_{d} \\
& +z_{2}^{T} g_{2}\left(x_{1}, x_{2}\right)(I-\sigma)\left[0, \tilde{v}_{\chi a(2)}^{T}(t)\right]^{T}+z_{2}^{T} g_{2}\left(x_{1}, x_{2}\right)(I-\sigma)\left[\tilde{v}_{\chi(31)}(t), 0, \tilde{v}_{\chi(33)}(t)\right]^{T} .
\end{aligned}
$$

With the possible actuator failure, $\dot{V}_{2}$ is no longer negative semidefinite, from which, we cannot guarantee system stability. We design an adaptive scheme to update the parameters $\operatorname{diag}\left\{\chi_{11}, \chi_{12}, \chi_{13}\right\}, \operatorname{diag}\left\{\chi_{21}, \chi_{22}\right\}, \operatorname{diag}\left\{\chi_{31}, \chi_{32}\right\}, \rho_{1(1)}, \rho_{1(2)}, \rho_{2(1)}$, and $\rho_{2(2)}$, and then, new Lyapunov functions can be used to ensure system stability.

\subsubsection{Adaptive Laws}

In this subsection, we develop an adaptive scheme for updating the parameter of the controller (4.80). The adaptive laws for $\operatorname{diag}\left\{\chi_{11}, \chi_{12}, \chi_{13}\right\}, \operatorname{diag}\left\{\chi_{21}, \chi_{22}\right\}$, and $\operatorname{diag}\left\{\chi_{31}, \chi_{32}\right\}$ can be generically chosen as

$$
\begin{aligned}
& \dot{\chi}_{1 j}=-\gamma_{1 j} z_{2}^{T} g_{2 j} \mu_{1 j} \\
& \dot{\chi}_{2 j}=-\gamma_{2 j} z_{2}^{T} g_{2(j+1)} \mu_{2 j} \\
& \dot{\chi}_{31}=-\gamma_{31} z_{2}^{T} g_{21} \mu_{31} \\
& \dot{\chi}_{32}=-\gamma_{33} z_{2}^{T} g_{23} \mu_{32},
\end{aligned}
$$

where $\gamma_{i j}>0$ is the adaptive gain, and

$$
\begin{aligned}
& \mu_{1}=\left[\mu_{11}, \mu_{12}, \mu_{13}\right]^{T}=h_{a} K_{a} \omega_{d} \\
& \mu_{2}=\left[\mu_{21}, \mu_{22}\right]^{T}=h_{2} K_{21} \omega_{d} \\
& \mu_{3}=\left[\mu_{31}, \mu_{32}\right]^{T}=h_{3} K_{31} \omega_{d},
\end{aligned}
$$


with $h_{a}, h_{2}, h_{3}, K_{a}, K_{21}$, and $K_{31}$ being the design matrices from the adaptive controller structure consisting of (4.81), (4.85), and (4.87).

Similarly, the adaptive laws for $\rho_{1(1)}, \rho_{1(2)}, \rho_{2(1)}$, and $\rho_{2(2)}$ can be chosen as

$$
\begin{aligned}
& \dot{\rho}_{1(j)}=-\Gamma_{(1 j)} f_{a j} z_{2}^{T} g_{2(j+1)} \phi_{1 j} \\
& \dot{\rho}_{2(1)}=-\Gamma_{(21)} f_{a 1} z_{2}^{T} g_{21} \phi_{21} \\
& \dot{\rho}_{2(2)}=-\Gamma_{(22)} f_{a 2} z_{2}^{T} g_{23} \phi_{22},
\end{aligned}
$$

where the parameter $\Gamma_{(i j)}=\Gamma_{(i j)}^{T}>0$ is the adaptive gain matrix. The function $\phi_{i}$ is defined as $\phi_{1}=\left[\phi_{11}, \phi_{12}\right]^{T}=h_{2} K_{22}$, and $\phi_{2}=\left[\phi_{21}, \phi_{22}\right]^{T}=h_{3} K_{31}$.

We then use a parameter projection scheme to ensure the boundedness of the estimated parameters in the presence of actuator failure uncertainties. We first consider the physical range of the indicator functions $\chi_{1}^{*}, \chi_{2}^{*}$, and $\chi_{3}^{*}$. From the definition of the indicator functions, we have

$$
0 \leq \chi_{1}^{*} \leq 1, \quad 0 \leq \chi_{2}^{*} \leq 1, \quad 0 \leq \chi_{3}^{*} \leq 1
$$

Because $\chi_{11}^{*}=\chi_{12}^{*}=\chi_{13}^{*}=\chi_{1}^{*}, \chi_{21}^{*}=\chi_{22}^{*}=\chi_{2}^{*}$, and $\chi_{31}^{*}=\chi_{32}^{*}=\chi_{3}^{*}$, we have $0 \leq \chi_{i j}^{*} \leq 1$. We can see that the estimated parameters $\chi_{i j}$ should also have the lower bound at 0 and the upper bound at 1 .

Let $\rho_{i k}^{* a}, \rho_{i k}^{* b}$ be the upper and lower bounds of the components $\rho_{i k}^{*}$ of $\rho_{i}^{*}, i=1,2$, $k=1,2, \ldots, k_{n}$, that is,

$$
\rho_{1 k}^{* a} \leq \rho_{1 k}^{*} \leq \rho_{1 k}^{* b}, \quad \rho_{2 k}^{* a} \leq \rho_{2 k}^{*} \leq \rho_{2 k}^{* b} .
$$

The upper and lower bounds $\rho_{i k}^{* a}, \rho_{i k}^{* b}$ can be obtained from the maximum and minimum values that each failing actuator can produce based on the actuator failure 
model (4.3).

With the upper and lower bounds of each component of $\rho_{i}$, we can see that each component of the estimated parameters $\rho_{i(j)}$ should also has the same boundaries as the component of $\rho_{i}$ because $\rho_{i(j)}$ is the estimate of $\rho_{i} \chi_{j}$ with $0 \leq \chi_{j} \leq 1$.

With the upper and lower bounds of the true parameters, we can modify the adaptive laws in (4.100) and (4.102), so that each component of the estimated parameters stays within its boundaries; and in particular, the initial conditions $\chi_{i j}(0), \rho_{i(j)}(0)$ are chosen inside the boundaries.

The adaptive laws for $\chi_{i j}(t)$ become

$$
\begin{aligned}
& \dot{\chi}_{1 j}=-\gamma_{1 j} z_{2}^{T} g_{2 j} \mu_{1 j}+\varrho_{\chi_{1} j}, \dot{\chi}_{2 j}=-\gamma_{2 j} z_{2}^{T} g_{2(j+1)} \mu_{2 j}+\varrho_{\chi_{2} j} \\
& \dot{\chi}_{31}=-\gamma_{31} z_{2}^{T} g_{21} \mu_{31}+\varrho_{\chi_{3} 1}, \quad \dot{\chi}_{32}=-\gamma_{33} z_{2}^{T} g_{23} \mu_{32}+\varrho_{\chi_{3} 2}
\end{aligned}
$$

where the projection function $\varrho_{\chi i j}$ is chosen as

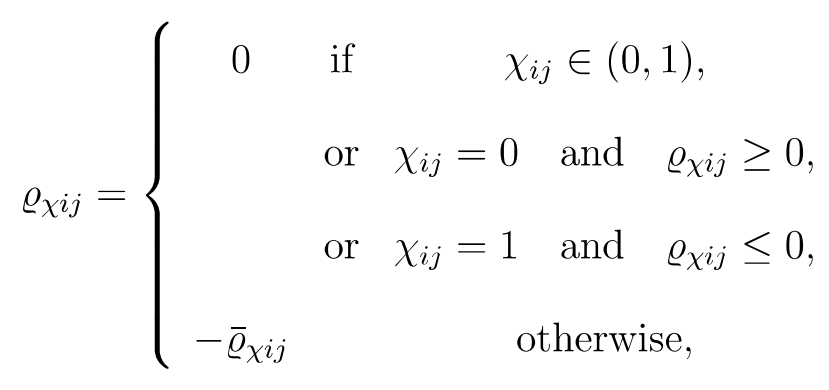

with $\bar{\varrho}_{\chi i j}$ defined as

$$
\begin{aligned}
& \bar{\varrho}_{\chi 1 j}=-\gamma_{1 j} z_{2}^{T} g_{2 j} \mu_{1 j} \\
& \bar{\varrho}_{\chi 2 j}=-\gamma_{2 j} z_{2}^{T} g_{2(j+1)} \mu_{2 j} \\
& \bar{\varrho}_{\chi 31}=-\gamma_{31} z_{2}^{T} g_{21} \mu_{31} \\
& \bar{\varrho}_{\chi 32}=-\gamma_{33} z_{2}^{T} g_{23} \mu_{32} .
\end{aligned}
$$


Similarly, the adaptive laws for $\rho_{i(j)}=\left[\rho_{i(j)(1)}, \ldots, \rho_{i(j)\left(n_{i}\right)}\right]^{T}$ become

$$
\begin{aligned}
& \dot{\rho}_{1(j)}=-\Gamma_{(1 j)} f_{a j} z_{2}^{T} g_{2(j+1)} \phi_{1 j}+\varrho_{\rho_{1(j)}} \\
& \dot{\rho}_{2(1)}=-\Gamma_{(21)} f_{a 1} z_{2}^{T} g_{21} \phi_{21}+\varrho_{\rho_{2(1)}} \\
& \dot{\rho}_{2(2)}=-\Gamma_{(22)} f_{a 2} z_{2}^{T} g_{23} \phi_{22}+\varrho_{\rho_{2(2)}},
\end{aligned}
$$

where the projection function $\varrho_{\rho i(j)}=\left[\varrho_{\rho i(j)(1)}, \ldots, \varrho_{\rho i(j)\left(n_{i}\right)}\right]^{T}$ is chosen as

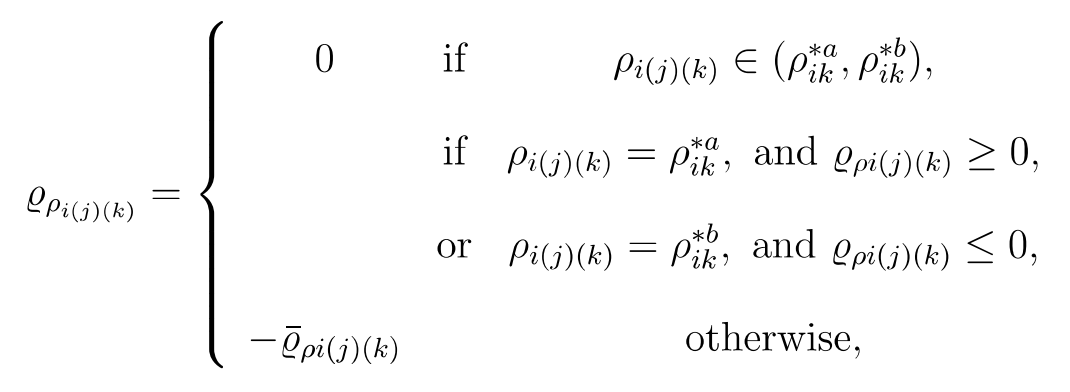

with $\bar{\varrho}_{\rho i(j)}=\left[\bar{\varrho}_{\rho i(j)(1)}, \ldots, \bar{\varrho}_{\rho i(j)\left(n_{i}\right)}\right]^{T}$ defined as

$$
\begin{aligned}
& \bar{\varrho}_{\rho 1(j)}=-\Gamma_{(1 j)} f_{a j} z_{2}^{T} g_{2(j+1)} \phi_{1 j} \\
& \bar{\varrho}_{\rho 2(1)}=-\Gamma_{(21)} f_{a 1} z_{2}^{T} g_{21} \phi_{21} \\
& \bar{\varrho}_{\rho 2(2)}=-\Gamma_{(22)} f_{a 2} z_{2}^{T} g_{23} \phi_{22} .
\end{aligned}
$$

It can be verified that the parameter projection schemes have the properties

$$
\begin{aligned}
\left(\chi_{i j}-\chi_{i j}^{*}\right) \varrho_{\chi_{i j}} & \leq 0 \\
\left(\rho_{i(j k)}-\chi_{i j}^{*} \rho_{i k}^{*}\right) \varrho_{\rho_{i(j k)}} & \leq 0
\end{aligned}
$$

With the parameter projection scheme and chosen initial conditions $0 \leq \chi_{i j}(0) \leq$ $1, \rho_{i k}^{* a} \leq \rho_{i(j k)}(0) \leq \rho_{i k}^{* b}$, we can guarantee the boundedness of every estimated parameter used in the adaptive control scheme. 


\subsubsection{Performance Analysis}

The adaptive actuator failure compensation scheme can ensure the stability and tracking of the system in any of the three cases of possible actuator failure patterns: no failure occurs, actuator $u_{1}$ failure case, or actuator $u_{2}$ failure case, as shown by the following theorem [18].

Theorem 1. The adaptive actuator failure compensation scheme with the feedback control law (4.80) updated by the adaptive laws (4.105)- (4.108), when applied to the robotic model (3.39)-(4.41), guarantees the closed-loop signal boundedness and asymptotic output tracking: $\lim _{t \rightarrow \infty}\left(y(t)-y_{m}(t)\right)=0$.

Proof. We first consider the error signals $z_{1}=x_{1}-y_{m}$, and $z_{2}=x_{2}-\beta$. With uncertain actuator failures, we have the derivatives of $z_{1}$ and $z_{2}$ as

$$
\begin{aligned}
& \dot{z}_{1}=-c_{1} z_{1}+f\left(x_{1}\right) z_{2} . \\
& \dot{z}_{2}=-f\left(x_{1}\right) z_{1}-c_{2} z_{2}+g_{2}\left(x_{1}, x_{2}\right)(I-\sigma)\left(v-v^{*}\right) .
\end{aligned}
$$

Because the derivative of the error signal $\dot{z}_{2}$ contains the actuator failure pattern $\sigma$ which changes according to each actuator failure case, we need to consider three Lyapunov function candidates $V_{a 1}, V_{a 2}, V_{a 3}$ for analyzing each actuator failure pattern to ensure the system stability.

We first consider the case of no actuator failure, with the Lyapunov function candidate

$$
\begin{aligned}
V_{a 1}= & \frac{1}{2} z_{1}^{T} z_{1}+\frac{1}{2} z_{2}^{T} z_{2}+\frac{1}{2} \sum_{i=1}^{3} \tilde{\chi}_{1 i}^{2} \gamma_{1 i}^{-1}+\frac{1}{2}\left[\sum_{i=1}^{2} \tilde{\chi}_{2 i}^{2} \gamma_{2 i}^{-1}+\sum_{i=1}^{2} \tilde{\chi}_{3 i}^{2} \gamma_{3 i}^{-1}\right] \\
& +\frac{1}{2}\left[\sum_{i=1}^{2} \tilde{\rho}_{1(i)}^{T} \Gamma_{1 i}^{-1} \tilde{\rho}_{1(i)}+\sum_{i=1}^{2} \tilde{\rho}_{2(i)}^{T} \Gamma_{2 i}^{-1} \tilde{\rho}_{2(i)}\right] .
\end{aligned}
$$


With the parameter projection scheme (83) and (84), we have the derivative of each term in (4.114) as

$$
\begin{aligned}
\frac{d}{d t} & \frac{1}{2}\left(z_{1}^{T} z_{1}+z_{2}^{T} z_{2}\right)=-c_{1} z_{1}^{T} z_{1}-c_{2} z_{2}^{T} z_{2}+z_{2}^{T} g_{2}\left(x_{1}, x_{2}\right)(I-\sigma) \tilde{v}_{\chi(1)} \\
& +z_{2}^{T} g_{2}\left(x_{1}, x_{2}\right)(I-\sigma)\left[0, \tilde{v}_{\chi a(2)}^{T}(t)\right]^{T}+z_{2}^{T} g_{2}\left(x_{1}, x_{2}\right)(I-\sigma)\left[\tilde{v}_{\chi(31)}(t), 0, \tilde{v}_{\chi(33)}(t)\right]^{T} \\
\frac{d}{d t}( & \left.\frac{1}{2} \sum_{i=1}^{3} \tilde{\chi}_{1 i}^{2} \gamma_{1 i}^{-1}\right)=-z_{2}^{T}\left[\sum_{i=1}^{3} \tilde{\chi}_{1 i} g_{2 i} \mu_{1 i}\right]+\sum_{i=1}^{3} \tilde{\chi}_{1 i} \gamma_{1 i}^{-1} \varrho_{\chi 1 i} \leq-z_{2}^{T} g_{2}\left(x_{1}, x_{2}\right) \tilde{v}_{\chi(1)} \\
\frac{d}{d t}( & \left.\frac{1}{2}\left(\sum_{i=1}^{2} \tilde{\chi}_{2 i}^{2} \gamma_{2 i}^{-1}+\sum_{i=1}^{2} \tilde{\rho}_{1(i)}^{T} \Gamma_{1 i}^{-1} \tilde{\rho}_{1(i)}\right)\right) \\
& =-z_{2}^{T} g_{2}\left(x_{1}, x_{2}\right)\left[0, \tilde{v}_{\chi a(2)}^{T}(t)\right]^{T}+\sum_{i=1}^{3} \tilde{\chi}_{2 i} \gamma_{2 i}^{-1} \varrho_{\chi 2 i}+\sum_{i=1}^{2} \tilde{\rho}_{1(i)}^{T} \Gamma_{1 i}^{-1} \varrho_{\rho_{1(i)}} \\
& \leq-z_{2}^{T} g_{2}\left(x_{1}, x_{2}\right)\left[0, \tilde{v}_{\chi a(2)}^{T}(t)\right]^{T} \\
\frac{d}{d t}( & \left.\frac{1}{2}\left(\sum_{i=1}^{2} \tilde{\chi}_{3 i}^{2} \gamma_{3 i}^{-1}+\sum_{i=1}^{2} \tilde{\rho}_{2(i)}^{T} \Gamma_{2 i}^{-1} \tilde{\rho}_{2(i)}\right)\right) \\
& =-z_{2}^{T} g_{2}\left(x_{1}, x_{2}\right)\left[\tilde{v}_{\chi(31)}(t), 0, \tilde{v}_{\chi(33)}(t)\right]^{T}+\sum_{i=1}^{3} \tilde{\chi}_{3 i} \gamma_{3 i}^{-1} \varrho_{\chi 3 i}+\sum_{i=1}^{2} \tilde{\rho}_{2(i)}^{T} \Gamma_{2 i}^{-1} \varrho_{\rho_{2(i)}} \\
& \leq-z_{2}^{T} g_{2}\left(x_{1}, x_{2}\right)\left[\tilde{v}_{\chi(31)}(t), 0, \tilde{v}_{\chi(33)}(t)\right]^{T} .
\end{aligned}
$$

Here we have used the parameter projection property (4.111) and (4.112); that is,

$$
\tilde{\chi}_{i j} \gamma_{i j}^{-1} \varrho_{\chi_{i j}} \leq 0 \quad \tilde{\rho}_{i(j)}^{T} \Gamma_{i j}^{-1} \varrho_{\rho_{i(j)}} \leq 0 .
$$

Based on equations (4.115)-(4.118) with $\sigma=\operatorname{diag}\{0,0,0\}$, the derivative of the Lyapunov function (4.114) becomes

$$
\dot{V}_{a 1} \leq-c_{1} z_{1}^{T} z_{1}-c_{2} z_{2}^{T} z_{2} \leq 0 .
$$

For the actuator $u_{1}$ failure case, we have the actuator failure pattern $\sigma=\operatorname{diag}\{1,0,0\}$. 
We need a new Lyapunov function candidate $V_{a 2}$ as

$$
\begin{aligned}
V_{a 2} & =\frac{1}{2} z_{1}^{T} z_{1}+\frac{1}{2} z_{2}^{T} z_{2}+\frac{1}{2}\left[\sum_{i=2}^{3} \tilde{\chi}_{1 i}^{2} \gamma_{1 i}^{-1}+\sum_{i=1}^{2} \tilde{\chi}_{2 i}^{2} \gamma_{2 i}^{-1}+\tilde{\chi}_{32}^{2} \gamma_{32}^{-1}\right. \\
& \left.+\sum_{i=1}^{2} \tilde{\rho}_{1(i)}^{T} \Gamma_{1 i}^{-1} \tilde{\rho}_{1(i)}+\tilde{\rho}_{2(2)}^{T} \Gamma_{22}^{-1} \tilde{\rho}_{2(2)}\right] .
\end{aligned}
$$

To obtain the derivatives of the Lyapunov function candidates in (4.121), we consider the derivative of each term similar to the derivation in the case of no actuator failure. In this case, we have

$$
\begin{aligned}
& \frac{d}{d t}\left(\frac{1}{2} \sum_{i=2}^{3} \tilde{\chi}_{1 i}^{2} \gamma_{1 i}^{-1}\right)=-z_{2}^{T} g_{2}\left(x_{1}, x_{2}\right)\left[0, \tilde{v}_{\chi a(2)}^{T}(t)\right]^{T}+\sum_{i=2}^{3} \tilde{\chi}_{2 i} \gamma_{2 i}^{-1} \varrho_{\chi_{2 i}} \\
& \quad \leq-z_{2}^{T} g_{2}\left(x_{1}, x_{2}\right)(I-\sigma) \tilde{v}_{\chi(1)} \\
& \frac{d}{d t}\left(\frac{1}{2}\left(\sum_{i=1}^{2} \tilde{\chi}_{2 i}^{2} \gamma_{2 i}^{-1}+\sum_{i=1}^{2} \tilde{\rho}_{1(i)}^{T} \Gamma_{1 i}^{-1} \tilde{\rho}_{1(i)}\right)\right) \\
& \quad=-z_{2}^{T} g_{2}\left(x_{1}, x_{2}\right)\left[0, \tilde{v}_{\chi a(2)}^{T}(t)\right]^{T}+\sum_{i=1}^{2} \tilde{\chi}_{2 i} \gamma_{2 i}^{-1} \varrho_{\chi_{2 i}}+\sum_{i=1}^{2} \tilde{\rho}_{1(i)}^{T} \Gamma_{1 i}^{-1} \varrho_{\rho_{1(i)}} \\
& \quad \leq-z_{2}^{T} g_{2}\left(x_{1}, x_{2}\right)(I-\sigma)\left[0, \tilde{v}_{\chi a(2)}^{T}(t)\right]^{T} \\
& \frac{d}{d t}\left(\frac{1}{2}\left(\tilde{\chi}_{32}^{2} \gamma_{32}^{-1}+\tilde{\rho}_{2(2)} \Gamma_{22}^{-1} \tilde{\rho}_{2(2)}^{T}\right)\right) \\
& \quad=-z_{2}^{T} g_{2}\left(x_{1}, x_{2}\right)\left[0, \tilde{v}_{\chi a(2)}^{T}(t)\right]^{T}+\tilde{\chi}_{32} \gamma_{32}^{-1} \varrho_{\chi 32}+\tilde{\rho}_{2(2)}^{T} \Gamma_{22}^{-1} \varrho_{\rho_{2(2)}} \\
& \quad \leq-z_{2}^{T} g_{2}\left(x_{1}, x_{2}\right)(I-\sigma)\left[\tilde{v}_{\chi(31)}(t), 0, \tilde{v}_{\chi(33)}(t)\right]^{T} .
\end{aligned}
$$

We notice that the terms $\tilde{\chi}_{11}^{2} \gamma_{11}^{-1}, \tilde{\chi}_{31}^{2} \gamma_{31}^{-1}$, and $\tilde{\rho}_{2(1)} \Gamma_{21}^{-1} \tilde{\rho}_{2(1)}$ disappear because $(I-\sigma)=\operatorname{diag}\{0,1,1\}$, with $\sigma=\operatorname{diag}\{1,0,0\}$. We can see that the terms in the derivatives of Lyapunov function candidates (4.122)-(4.124) cancel the non-negative semidefinite parts in (4.115), and the derivatives of the Lyapunov function (4.121) becomes

$$
\dot{V}_{a 2} \leq-c_{1} z_{1}^{T} z_{1}-c_{2} z_{2}^{T} z_{2} \leq 0
$$


For the actuator $u_{2}$ failure case, we have the actuator failure pattern $\sigma=\operatorname{diag}\{0,1,0\}$. We use a new Lyapunov function candidate

$$
\begin{aligned}
& V_{a 3}=\frac{1}{2} z_{1}^{T} z_{1}+\frac{1}{2} z_{2}^{T} z_{2}+\frac{1}{2}\left[\sum_{i=1,3} \tilde{\chi}_{1 i}^{2} \gamma_{1 i}^{-1}+\tilde{\chi}_{22}^{2} \gamma_{22}^{-1}\right. \\
& \left.+\sum_{i=1}^{2} \tilde{\chi}_{3 i}^{2} \gamma_{3 i}^{-1}+\tilde{\rho}_{1(2)}^{T} \Gamma_{12}^{-1} \tilde{\rho}_{1(2)}+\sum_{i=1}^{2} \tilde{\rho}_{2(i)}^{T} \Gamma_{2 i}^{-1} \tilde{\rho}_{2(i)}\right]
\end{aligned}
$$

With simular approach as actuator $u_{1}$ failure case, we can see that the terms $\tilde{\chi}_{12}^{2} \gamma_{12}^{-1}$, $\tilde{\chi}_{21}^{2} \gamma_{21}^{-1}$, and $\tilde{\rho}_{1(1)} \Gamma_{11}^{-1} \tilde{\rho}_{1(1)}$ disappear because $(I-\sigma)=\operatorname{diag}\{1,0,1\}$. In this case, we have

$$
\dot{V}_{a 3} \leq-c_{1} z_{1}^{T} z_{1}-c_{2} z_{2}^{T} z_{2} \leq 0
$$

The derivatives of our Lyapunov functions (4.120), (4.125), and (4.127) for all actuator failure cases are negative semidefinite. With the parameter projection schemes, we can show that all signals in the system are bounded. Since $z_{1}(t), z_{2}(t), \dot{z}_{1}(t) \in$ $L^{\infty} \bigcap L^{2}$, based on the Barbalat lemma we can conclude that $\lim _{t \rightarrow \infty} z_{1}=0 . \nabla \nabla \nabla$

From Theorem 1, we can see that the adaptive actuator failure compensation scheme can guarantee the asymptotic tracking for the system subject to uncertain actuator failures, that is, for the system operating in any of the three actuator failure cases.

\subsection{Initial Control Framework}

In this section, we discuss main ideas of an adaptive actuator failure compensation scheme with a general cooperative manipulator robotic system to develop an initial 
control framework used in this study.

A cooperative manipulator robotic system uses multiple manipulators to control an object. Since the movement of the object depends on the coordination of every manipulator, an actuator failure in the system may compromise the entire system. It is important to develop an actuator failure compensation scheme for the system. Because a cooperative manipulator robotic system contains many manipulators, it often has enough redundancy to allow for easy integration of the adaptive actuator failure compensation scheme.

\subsubsection{General Dynamic Model of Robotic System}

The dynamic model of a cooperative manipulator robotic system can be divided into two parts, which are the dynamics of each manipulator and the dynamic of the object.

In order to derive the dynamic model of a general cooperative manipulator robotic system, we first consider the dynamic of each manipulator. Since each robotic manipulator in the system can be viewed as a rigid robot interacting with the environment, we have the dynamic model of the $i$ th manipulator as

$$
D_{i}\left(q_{i}\right) \ddot{q}_{i}+C_{i}\left(q_{i}, \dot{q}_{i}\right) \dot{q}_{i}+G_{i}\left(q_{i}\right)=\tau_{i}-J_{i}^{T} \hat{\tau}_{i}
$$

where $q_{i}$ is the joint angle of robot $i, \tau_{i}$ is the vector of joint torques, and $\hat{\tau}_{i}$ donates the interacting force between robot endeffector and the object. $D_{i}\left(q_{i}\right)$ is the inertia matrix, $C_{i}\left(q_{i}, \dot{q}_{i}\right)$ is the Coriolis and centrifugal term, $G_{i}\left(q_{i}\right)$ is the gravity term, and $J_{i}$ is the Jacobian matrix from task space to Cartesian space.

We then obtain the dynamic model of the object. The general form of the dynamic model of a rigid object, which is interacting with the robotic manipulators, can be 
written as

$$
D_{o}\left(x_{o}\right) \ddot{x}_{o}+C_{o}\left(x_{o}, \dot{x}_{o}\right) \dot{x}_{o}+G_{o}\left(x_{o}\right)=\sum_{i=1}^{m} A_{i}^{T} \hat{\tau}_{i}
$$

where $x_{o}$ is the position and orientation of the platform, $m$ is the number of the manipulators in the system, $D_{o}\left(x_{o}\right)$ is the inertia matrix of the object, $C_{o}\left(x_{o}, \dot{x}_{o}\right)$ is the Coriolis and centrifugal term of the object, $G_{o}\left(x_{o}\right)$ is the gravity term of the object, and $A_{i}$ is the augmented Jacobian matrix of the object from the task space to Cartesian space.

Let $y_{i}$ be the coordinate of the endeffector of each manipulator, we have the relationship of each coordinate as

$$
\begin{aligned}
J_{i}\left(q_{i}\right) \dot{q}_{i} & =\dot{y}_{i} \\
A_{i}\left(x_{o}\right) \dot{x}_{o} & =\dot{y}_{i} .
\end{aligned}
$$

The general dynamic model of a cooperative manipulator robotic system can be obtained by combining (4.128) and (4.129). With the coordinate transformation, the dynamic equation of the cooperative robotic system can be written in the task space as

$$
D_{c}\left(x_{o}\right) \ddot{x}_{o}+C_{c}\left(x_{o}, \dot{x}_{o}\right) \dot{x}_{o}+G_{c}\left(x_{o}\right)=E^{T}\left(x_{o}\right) \tau,
$$

where the combine inertia matrix of the object and manipulators $D_{c}\left(x_{o}\right)$ is assumed to be a bounded and positive definite matrix, $C_{c}\left(x_{o}, \dot{x}_{o}\right)$ is the Coriolis and centrifugal term, $G_{c}\left(x_{o}\right)$ is the combined gravity term, $E=\left[E_{1}^{T}, \ldots, E_{m}^{T}\right]^{T}$ with $E_{i}\left(x_{o}\right)=$ $J_{i}^{-1} A_{i}, i=1,2, \ldots, m$, and $\tau=\left[\tau_{1}^{T}, \ldots, \tau_{m}^{T}\right]^{T}, \tau_{i}$ is the generalized torque vector of the $i$ th manipulator. 
With the general dynamic model of a cooperative manipulator system, we can design an adaptive actuator failure compensation scheme for the robotic system subject to uncertain actuator failures.

\subsubsection{Actuator Failure Compensation Scheme}

When an actuator failure occurs, the joint torque $\tau_{i}$ produced by that actuator becomes unknown. Because the complexity of the cooperative manipulator robotic system, it is difficult to identify failures in the system. In order to ensure the stability and tracking property of the system, we use the adaptive actuator failure compensation scheme developed in this study. We first rewrite the dynamic model in the state space form as

$$
\begin{aligned}
& \dot{x}_{1}=x_{2} \\
& \dot{x}_{2}=g_{1}\left(x_{1}, x_{2}\right)+g_{2}\left(x_{1}\right) u, y=x_{1},
\end{aligned}
$$

where $x_{1}=x_{o}$, and $x_{2}=\dot{x}_{o}, y$ is the output of the system, and $u=\tau$ is the input of the system. With the inertia matrix $D_{c}\left(x_{c}\right)$ nonsingular, we have

$$
\begin{aligned}
g_{1}\left(x_{1}, x_{2}\right) & =-D_{c}^{-1}\left(x_{1}\right)\left(C\left(x_{1}, x_{2}\right) x_{2}+G\left(x_{1}\right)\right) \\
g_{2}\left(x_{1}\right) & =D_{c}^{-1}\left(x_{1}\right) E^{T}\left(x_{1}\right) .
\end{aligned}
$$

We can design an adaptive actuator failure compensation scheme by the following design procedure:

(i) Obtain a desirable signal $\omega_{d}=g_{2}\left(x_{1}\right) u$ by using the backstepping design method, which can guarantee the closed-loop signal boundedness and asymptotic 
output tracking of the system in the absence of actuators failures. Define $z_{1}=$ $x_{1}-y_{m}, z_{2}=x_{2}-\beta$, where $y_{m} \in R^{n}$ is the desirable trajectory and $\beta=-c_{1} z_{1}+\dot{y}_{m}$. We have the desirable signal

$$
\omega_{d}=g_{2}\left(x_{1}, x_{2}\right) u=-g_{1}\left(x_{1}, x_{2}\right)-c_{2} z_{2}-z_{1}+\dot{\beta},
$$

(ii) Assuming all actuator failures are known, develop a nominal control for the system, which can produce a control signal $v$ such that $\omega_{d}=g_{2}\left(x_{1}\right) v$ for every desirable actuator failure pattern $\sigma$. The nominal controller structure can be written as

$$
v^{*}(t)=\sum_{i=1}^{n_{p}} \chi_{i}^{*} v_{(i)}^{*}(t)
$$

where $\chi_{i}^{*}$ is the indicator functions associated with each actuator failure pattern, the signal $v_{(i)}^{*}(t)$ is the nominal control signal for each case of actuator failure in the system, and $n_{p}$ is the number of the actuator failure patterns.

The control signal $v_{(i)}^{*}(t)$ can be designed such that $\omega_{d}=g_{2}\left(x_{1}, x_{2}\right) v_{(i)}^{*}(t)$. For each case of actuator failure,

$$
v^{*}(t)=v_{(i)}^{*}(t)=A v_{a(i)}^{* T}(t)
$$

where $A \in R^{m \times n}$ is the actuator failure pattern matrix, which is chosen to eliminate the failed actuator input from the equation since we do not have control over the failed actuator. The nominal control signal can be written as

$$
v_{a(i)}^{*}(t)=h_{i}\left(x_{1}, x_{2}\right) v_{0(i)}^{*}(t),
$$

where $h_{i}\left(x_{1}, x_{2}\right) \in R^{n \times n}$ is the design matrix. The signal $v_{0(i)}^{*}(t) \in R^{n \times 1}$ can be chosen 
to satisfy

$$
g_{2(i) 1}\left(x_{1}, x_{2}\right) \bar{u}_{i}+g_{2(i) 2} h_{i}\left(x_{1}, x_{2}\right) v_{0(i)}^{*}(t)=\omega_{d}
$$

where $g_{2(i) 1}$ is a submatrix of $g_{2}$, which only contain the column associated with the failed actuator, and $g_{2(i) 2}$ is a submatrix of $g_{2}$ without the column in $g_{2(i) 1}$.

(iii) Derive the adaptive controller structure for the system subject to uncertain actuator failure with the controller parameterization based on the nominal controller structure developed in part (ii). We can design the adaptive controller structure as

$$
v(t)=\sum_{i=1}^{n_{p}} v_{\chi(i)}(t)
$$

where $v_{\chi(i)}$ is the estimate of $\chi_{i}^{*} v_{(i)}^{*}$ with $\chi_{i}^{*}$ estimated by a diagonal matrix similar to what we did in Section $\mathrm{V}$ and $\rho_{i}^{*}$ is estimated by $\rho_{i}$ based on the actuator failure model (4.3).

(iv) Develop an adaptive scheme for updating the parameters used in the adaptive controller structure similar to what we did in Section V. For example, the adaptive laws for updating the parameter $\chi_{i j}$ can be chosen as

$$
\dot{\chi}_{i j}=-\gamma_{i j} z_{2}^{T} g_{2 j} \mu_{i j}
$$

where $\gamma_{i j}>0$ is the adaptive gain, and

$$
\mu_{i}=\left[\mu_{i 1}, \mu_{i 2}, \ldots, \mu_{i n}\right]^{T}=h_{i} K_{i 2} \omega_{d}
$$

with $h_{i}$, and $K_{i 2}$ being the design matrices from the adaptive controller structure. 
Similarly, the adaptive laws for $\rho_{i(j)}$ can be chosen as

$$
\dot{\rho}_{i(j)}=-\Gamma_{(i j)} f_{a j} z_{2}^{T} g_{2 j} \phi_{i j}
$$

where the parameter $\Gamma_{(i j)}=\Gamma_{(i j)}^{T}>0$ is the adaptive gain matrix. The function $\phi_{i}$ is defined as $\phi_{i}=\left[\phi_{i 1}, \phi_{i 2}, \ldots, \phi_{i n}\right]^{T}=h_{i} K_{i 1}$.

Such an adaptive actuator failure compensation scheme is applicable to specific system under this general cooperative robotic system model to improve the performance of various cooperative manipulator robotic system.

\subsection{Simulation Study}

In this section we simulate the adaptive actuator failure compensation control scheme based on the robotic model subject to uncertain actuator failures.

\subsubsection{System Model and Simulation Conditions}

The simulations are performed based on the dynamic model of the robotic system as

$$
\begin{aligned}
& \dot{x}_{1}=f\left(x_{1}\right) x_{2} \\
& \dot{x}_{2}=g_{1}\left(x_{1}, x_{2}\right)+g_{2}\left(x_{1}, x_{2}\right) u
\end{aligned}
$$

where $y=x_{1}$ is the output of the system. The matrix $f\left(x_{1}\right), g_{1}\left(x_{1}, x_{2}\right)$, and $g_{2}\left(x_{1}, x_{2}\right)$ are calculated from equation (10). The simulations assign the mass of each actuator as $m_{1}=m_{2}=m_{3}=1 \mathrm{~kg}$, the mass of the platform $m_{p}=10 \mathrm{~kg}$, the moment of inertia of the platform $I_{p}=1 \mathrm{~kg} \cdot \mathrm{m}^{2}$, and the length of the platform $b_{0}=1 \mathrm{~m}$. For simulation we choose $c_{1}=c_{2}=2, \gamma_{i j}=0.01, \Gamma_{i(j)}=0.01 I_{2}, f_{a i}=[1, \sin 0.3 t]^{T}$. The 
initial conditions are chosen as $y(0)=[1,1]^{T}, \chi_{i j}(0)=0$, and $\chi_{i(j)}=[0,0]^{T}$.

In this simulation study, two simulations are evaluated as follows:

Lock In-Place Failure of the Actuator. In this simulation, we use a constant reference output $y_{m}(t)=[2,0.5]^{T}$. We simulate actuator failures occur twice as follows:

(i) No actuator failure case: $u(t)=v(t)$ for $0 \leq t<50 \mathrm{~s}$,

(ii) Actuator $u_{2}$ failure case: $u_{2}=\bar{u}_{2}=20$ at time $50 \leq t<100 \mathrm{~s}$,

(iii) The failing actuator $u_{2}$ becomes normal again, no actuator failure case: $u(t)=$ $v(t)$ for $100 \leq t<150 s$,

(iv) Actuator $u_{1}$ failure case: $u_{1}=\bar{u}_{1}=-30$ at time $150 \leq t<200 \mathrm{~s}$.

Sinusoidal Actuator Failure. In this case, we use a sinusoidal reference output $y_{m}(t)=[2+\sin (0.1 t), 0.2+0.1 \sin (0.1 t)]^{T}$. We let actuator failures happen two times as follow:

(i) No actuator failure case: $u(t)=v(t)$ for $0 \leq t<50 s$,

(ii) Actuator $u_{2}$ failure case: $u_{2}=\bar{u}_{2}=20 \sin (0.3 t)$ at time $50 \leq t<100 \mathrm{~s}$,

(iii) The failing actuator $u_{2}$ becomes normal again, no actuator failure case: $u(t)=$ $v(t)$ for $100 \leq t<150 s$,

(iv) Actuator $u_{1}$ failure case: $u_{1}=\bar{u}_{1}=-30 \sin (0.3 t)$ at time $150 \leq t<200 s$.

\subsubsection{Simulation Results}

For the Lock In-Place Failure of the Actuator case, the simulation results show the output of the system in Figure 4.2, the control input in Figure 4.3, and the adaptive parameters are presented in Figure 4.4 - Figure 4.5.

For the Sinusoidal Actuator Failure case, the simulation results show the output of 
the system in Figure 4.6, the control input in Figure 4.7, and the adaptive parameters are presented in Figure 4.8 - Figure 4.9.
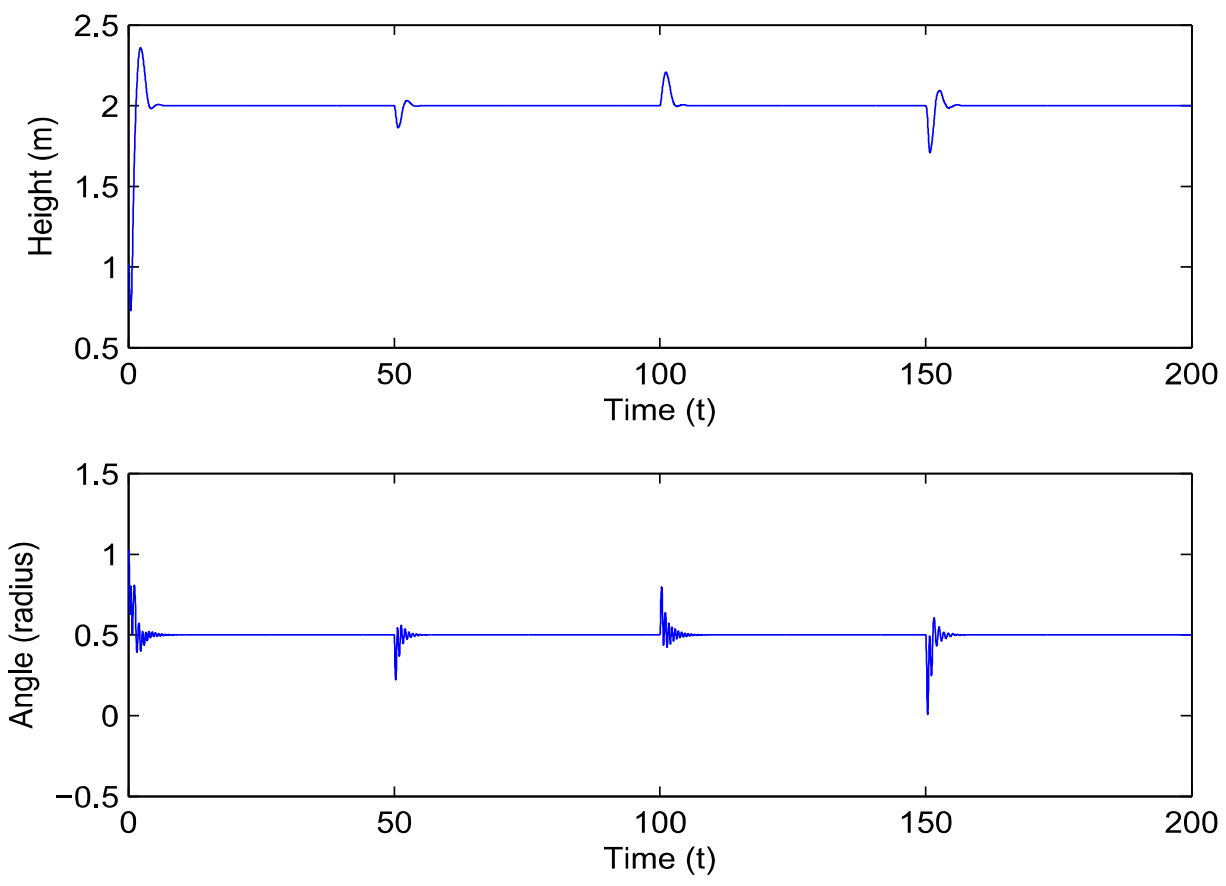

Figure 4.2: System responses for $y_{m}(t)=[2+\sin (0.1 t), 0.5]^{T}$ with constant failure. 

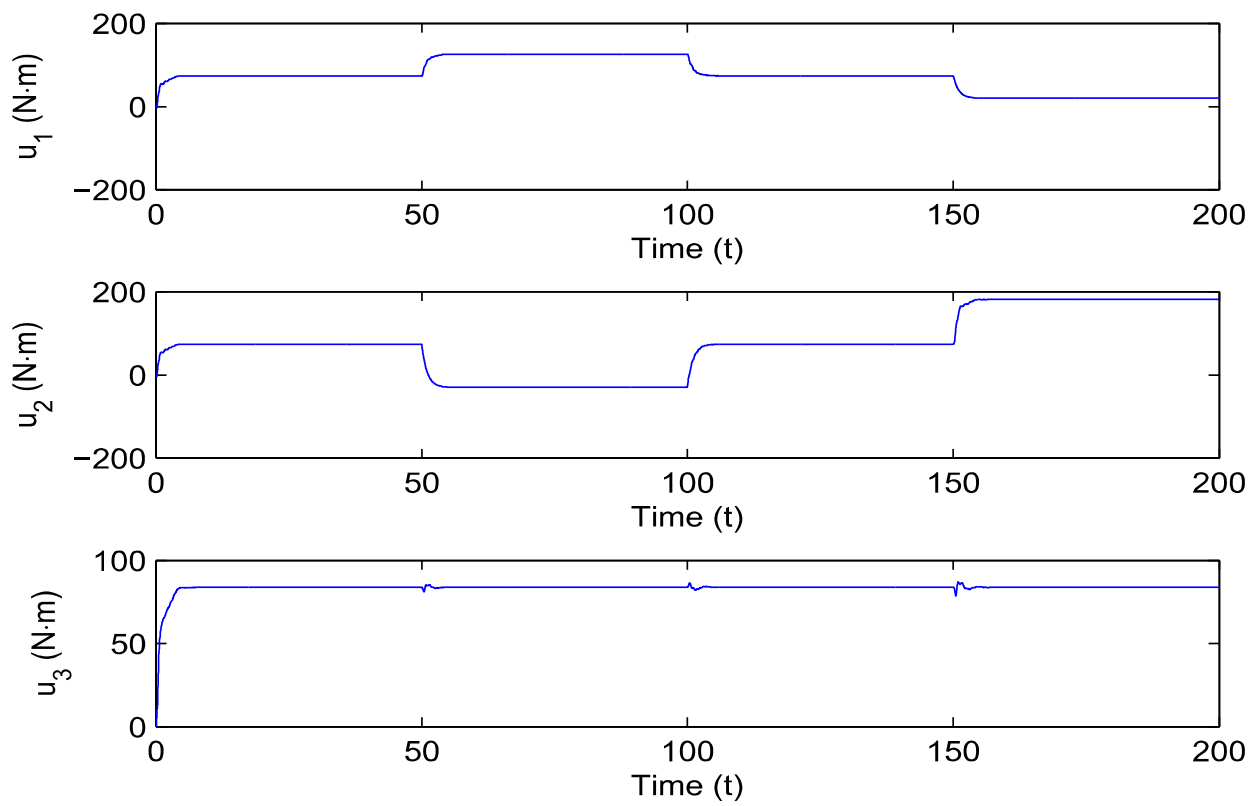

Figure 4.3: Actuator outputs subject to constant actuator failure.
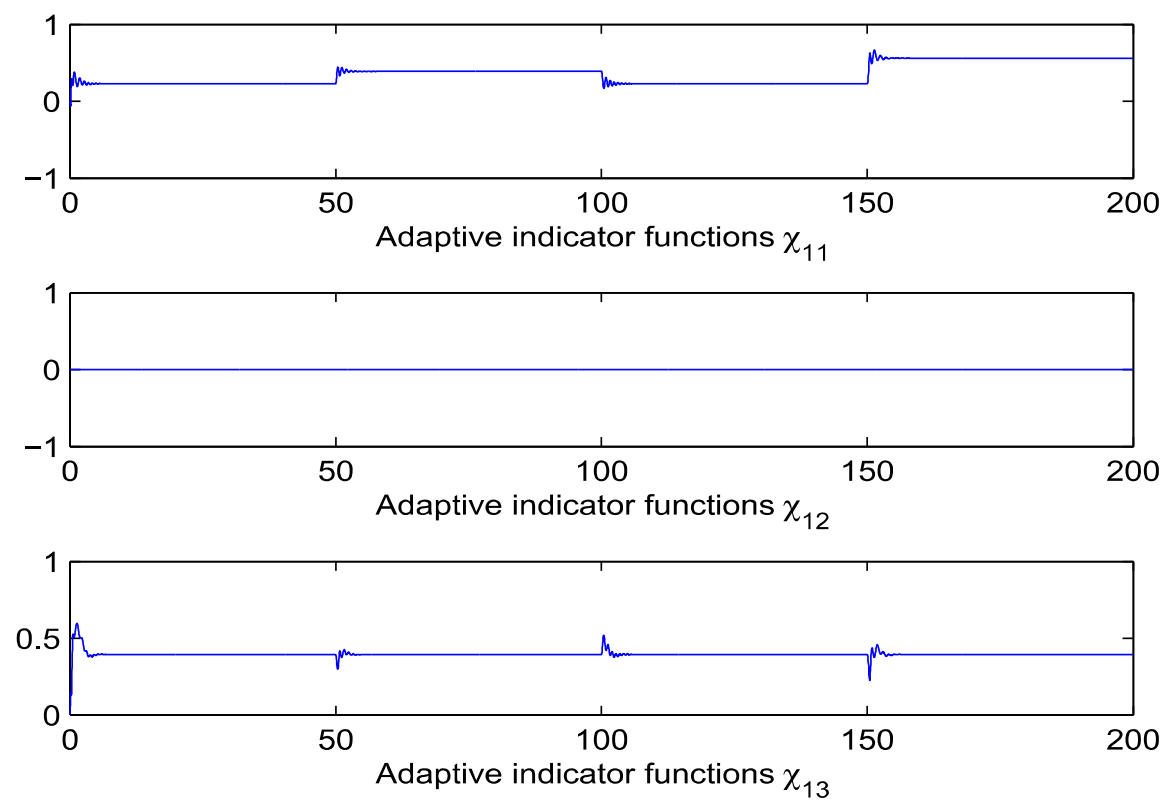

Figure 4.4: Adaptive indicator functions $\chi_{11}, \chi_{12}, \chi_{13}$. 

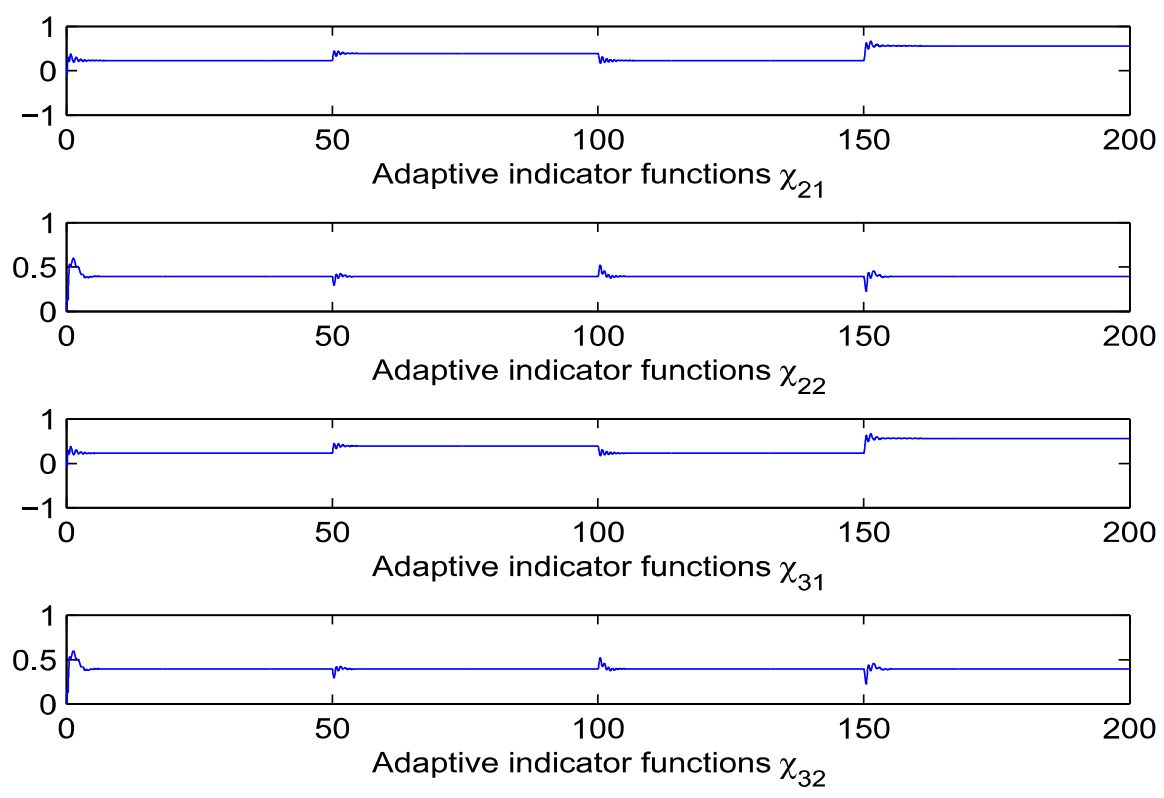

Figure 4.5: Adaptive indicator functions $\chi_{21}, \chi_{22}, \chi_{31}, \chi_{32}$.
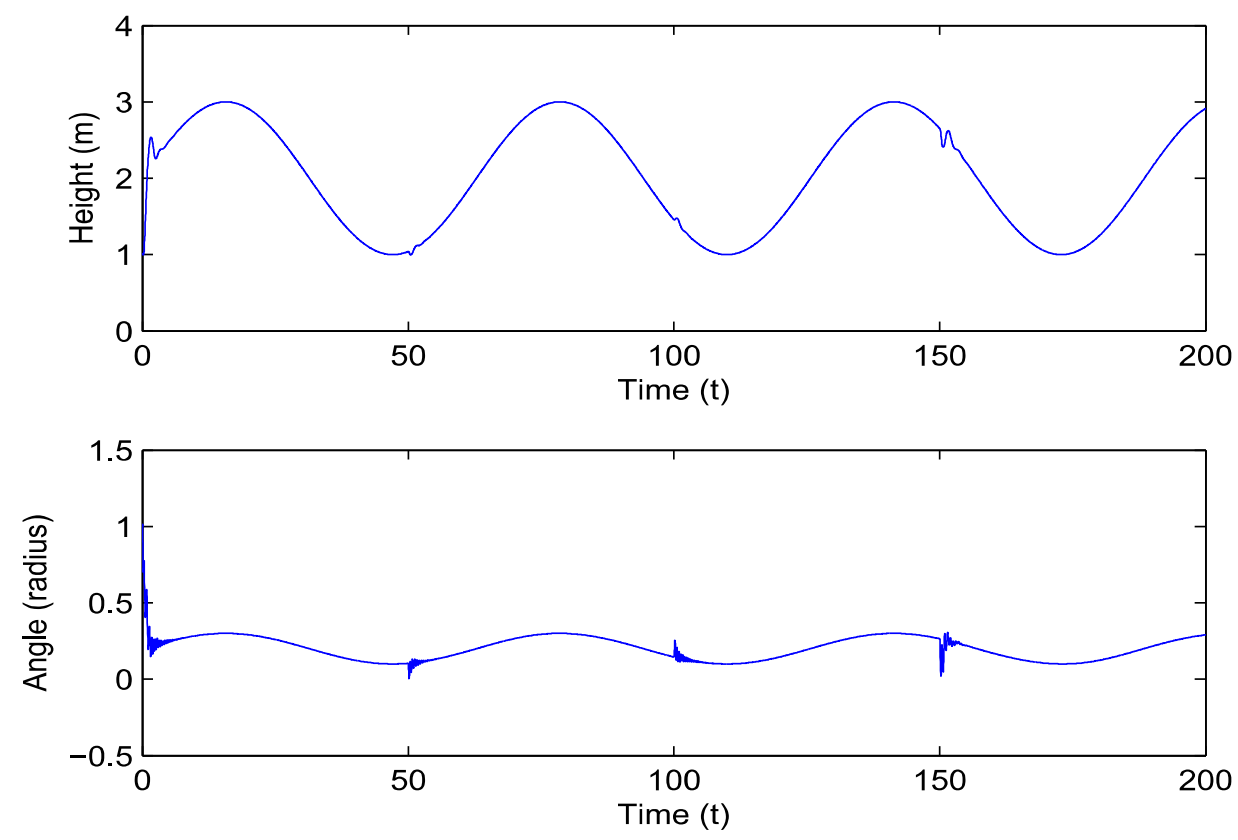

Figure 4.6: System responses for $y_{m}(t)=[2+\sin (0.1 t), 0.2+0.1 \sin (0.1 t)]^{T}$ with sinusoidal actuator failure. 

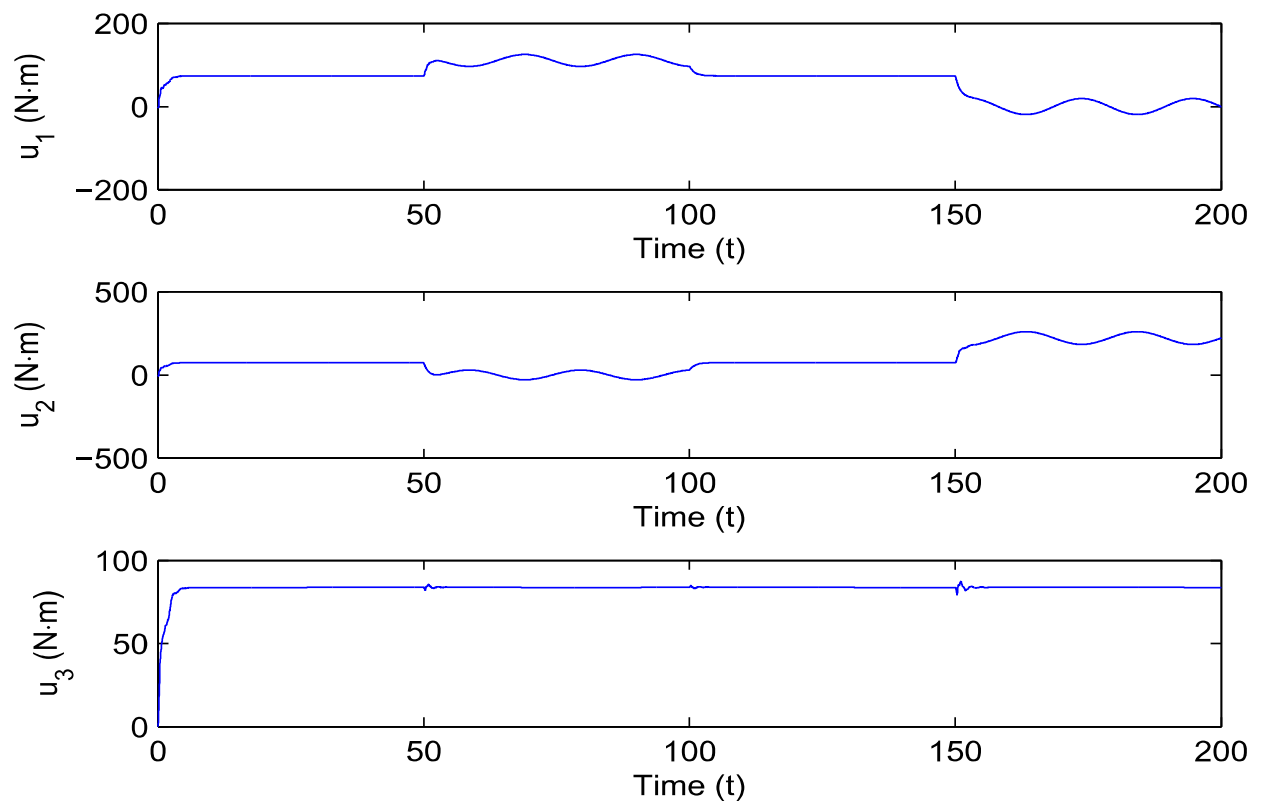

Figure 4.7: Actuator outputs subject to sinosoidal actuator failure.
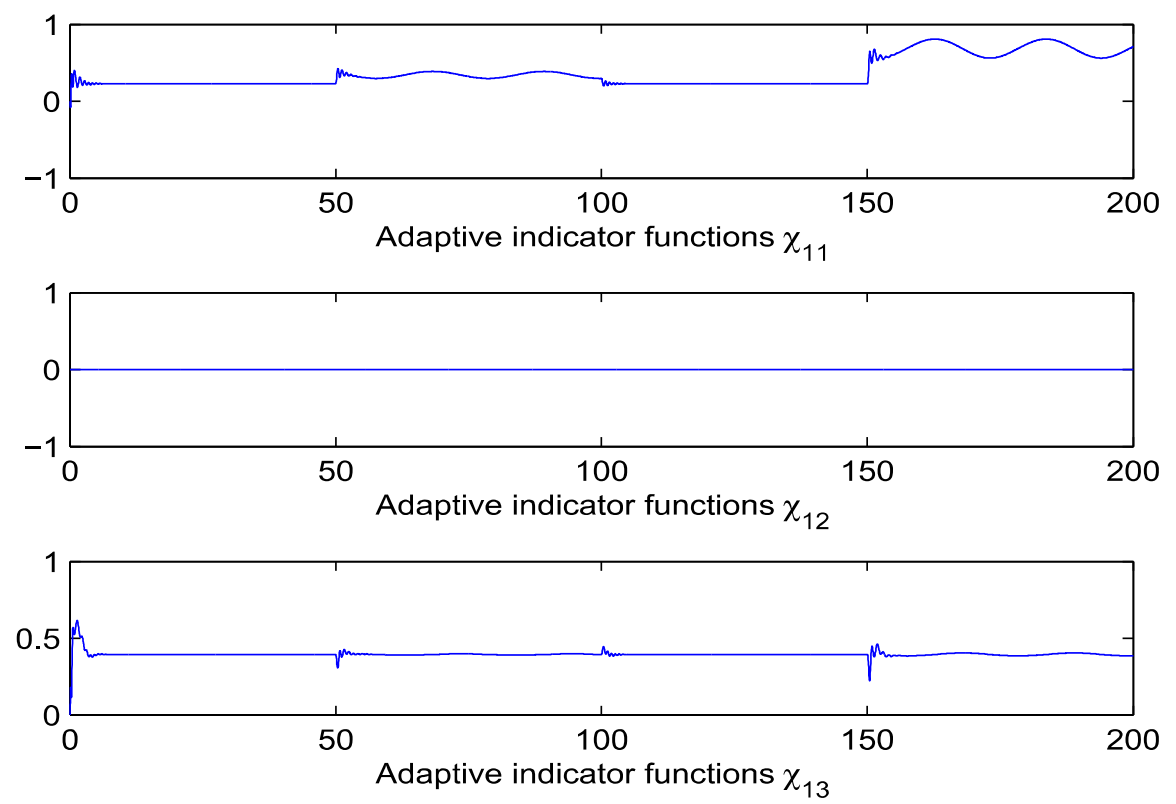

Figure 4.8: Adaptive indicator functions $\chi_{11}, \chi_{12}, \chi_{13}$. 

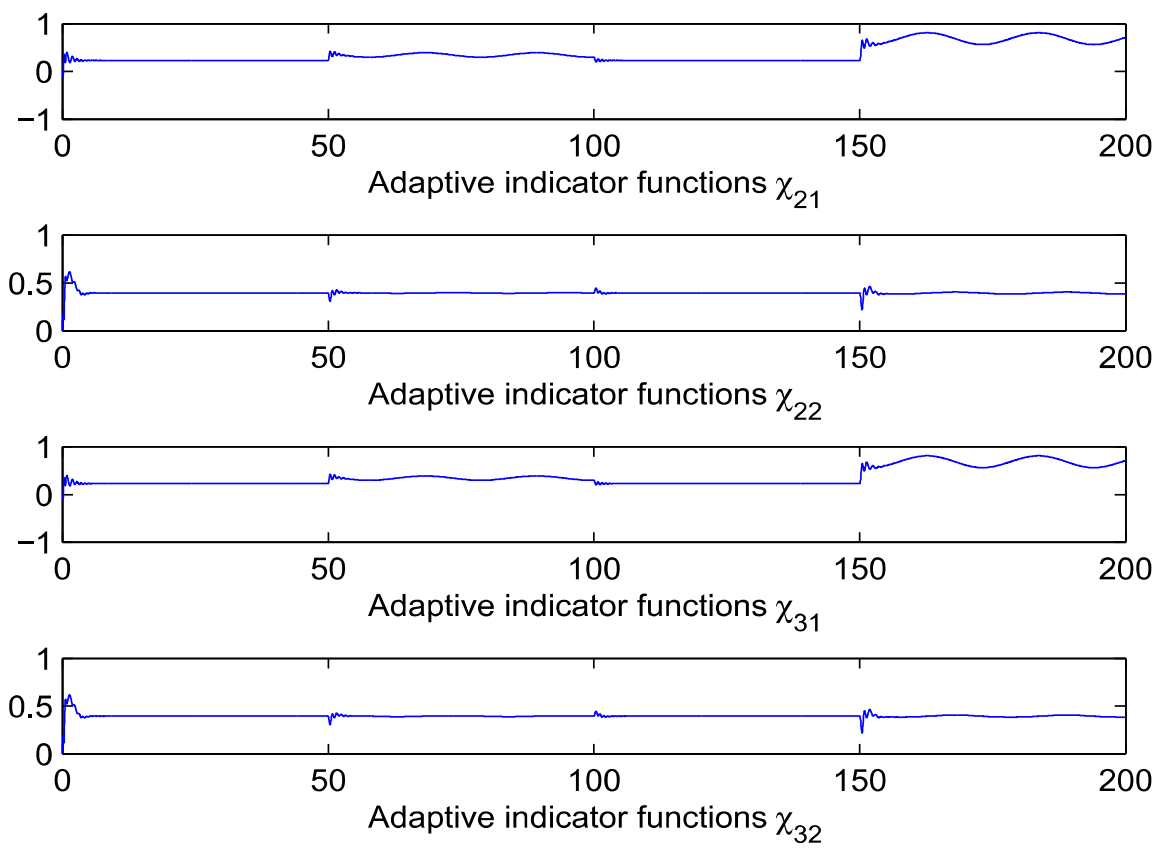

Figure 4.9: Adaptive indicator functions $\chi_{21}, \chi_{22}, \chi_{31}, \chi_{32}$.

The simulation results confirm that the adaptive actuator failure compensation scheme can guarantee that the tracking error of the system goes to zero as time goes to infinity for a constant or a sinusoidal desirable trajectory. The adaptive actuator failure compensation scheme allows one actuator $u_{1}$ or $u_{2}$ to fail, but both actuators cannot fail at the same time. Finally, the simulation results show that adaptive actuator failure compensation scheme supports both a constant and a sinusoidal failure signal.

\subsection{Conclusion}

Cooperative manipulator robotic systems which perform tasks such as moving objects, aiming an automatic firearm, and controlling a laser beam, are crucial for many 
operations that work in an extreme environment. Since the systems contain multiple manipulators, actuator redundancy usually exists which is required for actuator failure compensation. Developing an adaptive actuator failure compensation scheme that can effectively compensate for the actuator failures in the system is important for the operations and is a challenging control problem.

In this chapter, we have studied a benchmark cooperative manipulator system, and then developed a new adaptive actuator failure compensation scheme for a class of nonlinear multi-input multi-output cooperative manipulator robotic system. Our research shows that, with a complete parameterization of failure pattern, a desirable closed-loop stability and asymptotic tracking of the system can be achieved, despite the uncertain actuator failures. The simulation results also verified the performance of the adaptive control algorithm when applied to the benchmark robotic model subject to uncertain actuator failure. 


\section{Chapter 5}

\section{Design for A Hexapod System}

In this chapter, we develop a new adaptive actuator failure compensation scheme based on the initial adaptive actuator failure compensation framework to control a Hexapod system. The control design ensures desired closed-loop stability and asymptotic output tracking of the system subject to uncertain actuator failure.

Hexapod robotic systems play an important role in several applications such as precision pointing systems, where Hexapods are used to aim laser devices. The Hexapod has six manipulators to collectively move the payload, but we may not need to control six degrees of freedom at all time. The Hexapod needs three actuators to control the orientation of the payload, so it is possible to use the remaining actuators to compensate for failures in the system.

In this study, we develop a new adaptive actuator failure compensation scheme to control a Hexapod. The control design ensures desired closed-loop stability and asymptotic output tracking of the system subject to uncertain actuator failures. In this study, we also consider the failure cases such that multiple actuator failures occur at the same time. This study makes the following contributions to the robotics and control fields:

- Develop an adaptive actuator failure compensation scheme for a Hexapod subjected to uncertain actuator failure without explicit knowledge of the failing actuator. 
- Extend the scope of the adaptive actuator failure control design method to actuator failure cases where more than one actuator fails at the same time (concurrent actuator failures).

- Study the simulation of the adaptive actuator failure compensation scheme based on the Hexapod model subject to uncertain actuator failure.

The chapter is organized as follows. In Section II, we derive the dynamic model of the Hexapod to be used in this study. In Section III, we formulate the problem with the discussion of the control objective, actuator failure model and concurrent actuator failures. In Section IV, we design a nominal controller for the system in a nominal situation in which the knowledge of actuator failure is known. In Section V, the adaptive control scheme is developed based on the nominal controller structure, for the case of uncertain actuator failure. Finally in Section VI, the simulation results are presented to confirm the effectiveness of the design.

\subsection{System Modeling of the Hexapod System}

In this study, we will develop an adaptive actuator failure compensation control solution framework of a Hexapod in the presence of uncertain actuator failures. We consider the Hexapod and the manipulator structure in Figure 5.1.

\subsubsection{Dynamic Model of the Hexapod}

The dynamic equation of the $i$ strut of the Hexapod is given in the joint space as [11]

$$
\begin{aligned}
m_{i} d_{i}^{T} \ddot{p}_{s i}= & f_{m i}-f_{p i}-m_{i} d_{i}^{T} g-k_{i}\left(d_{i}^{T}\left(p_{s i}-q_{i}\right)-l_{r i}\right) \\
& -b_{i} d_{i}^{T}\left(\dot{p}_{s i}-\dot{q}_{i}\right), i=1,2, \ldots 6,
\end{aligned}
$$




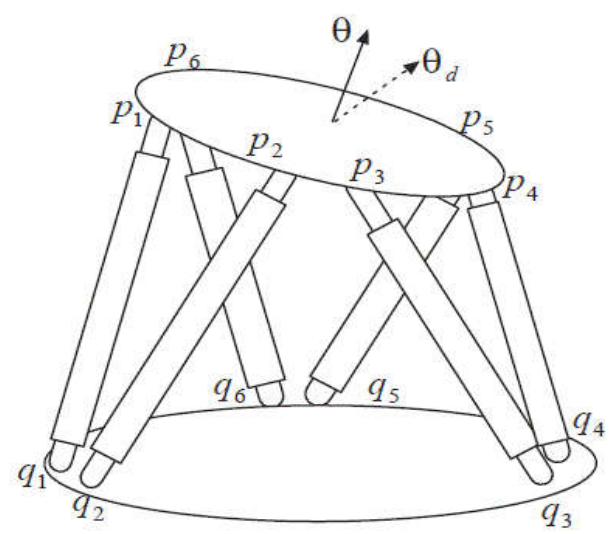

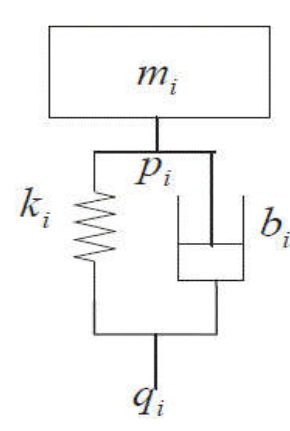

(a)

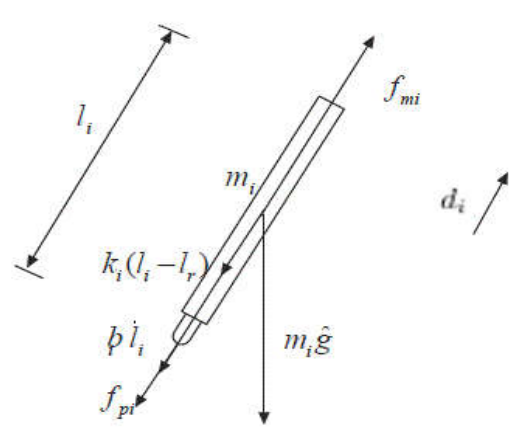

(b)

Figure 5.1: The model of Hexapod and the manipulator structure.

where $m_{i}$ is the mass of the $i$ th strut, $g$ is the gravitational constant, $k_{i}$ is the constant of stiffness of the $i$ th strut, $b_{i}$ is the damping constant of the $i$ th strut, $f_{m i}$ is the force of the motor which located in the top of the $i$ th strut, $f_{p i}$ is the force of the $i$ th strut exerted on the platform, $l_{r i}$ denotes the relaxed strut length, $d_{i} \in R^{3}$ is the unit direction along the $i$ th strut, $p_{s i}, \dot{p}_{s i}, \ddot{p}_{s i} \in R^{3}$ represents the position, velocity and acceleration of the top of the $i$ th strut respectively and $q_{i}, \dot{q}_{i} \in R^{3}$ represents the position and velocity of the bottom of the $i$ th strut.

Define $l=\left[l_{1}, l_{2}, \ldots, l_{6}\right]^{T}$, we can combine the six equations of struts in (5.1) as

$$
M_{s} \ddot{l}+B \dot{l}+K\left(l-l_{r}\right)+M_{s} q_{a u}+M_{s} g_{u}=f_{m}-f_{p}
$$

where $M_{s}=\operatorname{diag}\left\{m_{1}, m_{2}, \ldots, m_{6}\right\}, B=\operatorname{diag}\left\{b_{1}, b_{2}, \ldots, b_{6}\right\}, K=\operatorname{diag}\left\{k_{1}, k_{2}, \ldots, k_{6}\right\}$, $l_{r}=\left[l_{r 1}, l_{r 2}, \ldots, l_{r 6}\right]^{T}, q_{a u}=\left[d_{1}^{T} \ddot{q}_{1}, d_{2}^{T} \ddot{q}_{2}, \ldots, d_{6}^{T} \ddot{q}_{6}\right]^{T}, g_{u}=\left[d_{1}^{T} g, d_{2}^{T} g, \ldots, d_{6}^{T} g\right]^{T}, f_{m}=$ $\left[f_{m 1}, f_{m 2}, \ldots, f_{m 6}\right]^{T}$, and $f_{p}=\left[f_{p 1}, f_{p 2}, \ldots, f_{p 6}\right]^{T}$ are the combined matrices.

The equation describing the motion of the orientation of the platform, which is 
modelled as a rigid body, is given in the reference frame by

$$
I_{p} \ddot{\theta}+C_{p}(\theta, \dot{\theta}) \dot{\theta}=\tau
$$

where $\theta \in R^{3}$ is the angular displacement of the payload, $I_{p} \in R^{3 \times 3}$ is the matrix of moment of inertia, $C_{p}(\theta, \dot{\theta}) \dot{\theta}$ represents the Coriolis and centripetal terms and $\tau \in R^{3}$ is the generalized torque exerted on the platform.

Let $\chi_{b}=\left[\theta_{b}^{T}, p_{b}^{T}\right] \in R^{6}$ denote the pose of the base frame with respect to the reference frame, where $\theta_{b}^{T} \in R^{3}$ represents the angular displacement and $p_{b} \in R^{3}$ is the position of the base frame. The generalized torque can be calculated from the strut forces as

$$
\tau=E^{T}\left(\theta, \chi_{b}\right) f_{p}
$$

where the transformation matrix $E\left(\theta, \chi_{b}\right)=J\left(\theta, \chi_{b}\right) R^{T}, J\left(\theta, \chi_{b}\right)$ is a $6 \times 3$ matrix which is a part of the manipulator Jacobian and $R^{T}$ is a $3 \times 3$ rotation matrix from the base frame to the reference frame.

Combining (5.1) and (5.3) as in [11], we have the dynamic of the Hexapod as

$$
\begin{aligned}
D\left(\theta, \chi_{b}\right) \ddot{\theta}+C\left(\theta, \chi_{b}, \dot{\theta}, \dot{\chi_{b}}\right) \dot{\theta}+G\left(\theta, \chi_{b}\right) \\
=E^{T} f_{m}-P\left(\theta, \chi_{b}, \dot{\theta}, \dot{\chi}_{b}\right) \theta-E^{T} K\left(\bar{\pi}\left(\theta, \chi_{b}\right)-l_{r}\right) \\
\quad-\bar{C}\left(\theta, \chi_{b}, \dot{\chi}_{b}\right) \dot{\chi}_{b}-\bar{Q}\left(\theta, \chi_{b}, \dot{\chi}_{b}\right) \ddot{\chi}_{b},
\end{aligned}
$$

where $\bar{\pi}$ is a constraint function and

$$
\begin{aligned}
& D\left(\theta, \chi_{b}\right)=I_{p}+E^{T} M_{s} E, C\left(\theta, \chi_{b}, \dot{\theta}, \dot{\chi}_{b}\right)=C_{p}+E^{T} B E+E^{T} M_{s} \dot{J} R^{T}+2 E^{T} M_{s} P_{1}, \\
& G\left(\theta, \chi_{b}\right)=E^{T} M_{s} g_{u}, P\left(\theta, \chi_{b}, \dot{\theta}, \dot{\chi}_{b}\right)=E^{T} M_{s} P_{2}+E^{T} B P_{3}, \\
& \bar{Q}\left(\theta, \chi_{b}, \dot{\chi}_{b}\right)=E^{T} M_{s} J_{B}+E^{T} M_{s}\left[Q \quad 0_{6 \times 3}\right],
\end{aligned}
$$




$$
\begin{aligned}
& \bar{C}\left(\theta, \chi_{b}, \dot{\chi}_{b}\right)=E^{T} M_{s} C_{B} \\
& P_{1}\left(\theta, \chi_{b}, \dot{\chi}_{b}\right) \dot{\theta}=J\left(\theta, \chi_{b}\right) \dot{\theta}_{b} \times R^{T}\left(\chi_{b}, \dot{\chi}_{b}\right) \dot{\theta} \\
& P_{2}\left(\theta, \dot{\theta}, \chi_{b}, \dot{\chi}_{b}\right) \theta=\dot{J}\left(\theta, \chi_{b}, \dot{\theta}, \dot{\chi}_{b}\right) \theta \times R^{T}\left(\chi_{b}, \dot{\chi}_{b}\right) \dot{\theta}+J\left(\theta, \chi_{b}\right) \dot{\theta}_{b} \times \dot{\theta}_{b} \times R^{T}\left(\chi_{b}, \dot{\chi}_{b}\right) \theta \\
& P_{3}\left(\theta, \chi_{b}, \dot{\chi}_{b}\right) \theta=J\left(\theta, \chi_{b}\right) \dot{\theta}_{b} \times R^{T}\left(\chi_{b}, \dot{\chi}_{b}\right) \theta \text { and } \\
& Q\left(\theta, \chi_{b}, \dot{\chi}_{b}\right) \ddot{\theta}=J\left(\theta, \chi_{b}\right) \ddot{\theta}_{b} \times R^{T}\left(\chi_{b}, \dot{\chi}_{b}\right) \theta
\end{aligned}
$$

\subsubsection{Dynamic Model in State Space Form}

The Hexapod model (5.5) can be written in the state space form as

$$
\dot{x}_{1}=x_{2}, \quad \dot{x}_{2}=g_{1}\left(x_{1}, x_{2}\right)+g_{2}\left(x_{1}, x_{2}\right) u(t),
$$

where $x_{1}=\theta \in R^{3}$ and $x_{2}=\dot{\theta} \in R^{3}$ are the states of the system, $y=x_{1} \in R^{3}$ is the output of the system, $u=f_{m} \in R^{6}$ is the system input, $g_{1} \in R^{3}$, and $g_{2} \in R^{3 \times 6}$ are known matrices based on the dynamic equation in (5.5)

$$
\begin{aligned}
g_{2}\left(x_{1}, x_{2}\right)= & D\left(\theta, \chi_{b}\right)^{-1} E^{T} \\
g_{1}\left(x_{1}, x_{2}\right)= & D\left(x_{1}, \chi_{b}\right)^{-1}\left[-C\left(x_{1}, \chi_{b}, x_{2}, \dot{\chi}_{b}\right) \dot{\theta}-G\left(x_{1}, \chi_{b}\right)\right. \\
& -P\left(x_{1}, \chi_{b}, \dot{\theta}, \dot{\chi}_{b}\right) x_{1}-E^{T} K\left(\bar{\pi}\left(x_{1}, \chi_{b}\right)-l_{r}\right) \\
& \left.-\bar{C}\left(x_{1}, \chi_{b}, \dot{\chi}_{b}\right) \dot{\chi}_{b}-\bar{Q}\left(x_{1}, \chi_{b}, \dot{\chi}_{b}\right) \ddot{\chi}_{b}\right] .
\end{aligned}
$$

In order to design an adaptive control design, we first assume that the base position $\chi_{b}$ and the base velocity $\dot{\chi}_{b}$ are measured. Under this assumption the Jacobian matrix $J$, the rotation matrix $R$ and the constraint function $\bar{\pi}$ are known. 


\subsection{Problem Formulation}

The objective of this research is to design an adaptive actuator failure compensation scheme to ensure closed-loop stability and asymptotic output tracking of a Hexapod without explicit knowledge of failing actuators.

\subsubsection{Control Objective}

Hexapods are used in applications that require high precision as well as reliability such as controlling lasers in a surgery or carrying sensitive materials. Since a Hexapod contains many actuators, the possibility of actuator failure is relatively high. For example, a damage in a single wire may cause an actuator to completely fail, or a delay in the communication between the controller and an actuator may cause the output of that actuator to remain constant for a period of time. To solve these problems, we design a controller, which utilizes redundancy in the system to compensate for the failures. In this study, we design an adaptive actuator failure compensation for the Hexapod to control the orientation of the platform despite concurrent failures.

The control objective of the study can be described mathematically as follows. Consider the Hexapod model (5.6), due to possible actuator failures, an applied feedback control signal $v(t)=\left[v_{1}, v_{2}, \ldots, v_{6}\right]^{T}$ may not reach the system, as $u_{j}(t)$ is not equal to $v_{j}(t)$. More precisely, in the presence of failures, the signal $u(t)$ is

$$
u(t)=(I-\sigma(t)) v(t)+\sigma(t) \bar{u}
$$

where $\bar{u}$ is an unknown failure signal and $\sigma(t)=\operatorname{diag}\left\{\sigma_{1}, \sigma_{2}, \ldots, \sigma_{6}\right\}$ is the actuator failure pattern matrix such that $\sigma_{i}(t)=1$ if the $i$ actuator fails and $\sigma_{i}(t)=0$ otherwise. 
The control objective is to design a feedback control signal $v(t)$ such that $\lim _{t \rightarrow \infty}\left(g_{2}\left(x_{1}, x_{2}\right) u(t)-\omega_{d}(t)\right)=0$, despite the uncertain actuator failures $u(t)=$ $(I-\sigma(t)) v(t)+\sigma(t) \bar{u}$, where $\omega_{d}$ is a desirable control signal for $\omega$ to control the system (5.6). The controller is designed to make all signals in the closed-loop system to be bounded and the output $y(t)=x_{1}(t)$ asymptotically tracks a given reference signal $y_{m}(t)$. When this objective is met, the real system $\dot{x}_{1}=x_{2}, \dot{x}_{2}=g_{1}\left(x_{1}, x_{2}\right)+$ $g_{2}\left(x_{1}, x_{2}\right) u(t)$ will be ensured to have the desired signal boundedness and tracking properties, and this will be achieved through control adaptation.

The adaptive actuator failure compensation scheme is designed to compensate for failures in the system without the knowledge of the failing actuator $j$, time that actuator failure occurs $t_{j}$, or the output of the failing actuator $\bar{u}_{j}(t)$.

\subsubsection{Actuator Failure Model}

In this study we assume that we do not have the exact knowledge of the actuator failures in the system. Let $u(t)=f_{m}(t) \in R^{6}$ be the system input vector. When an actuator failure occurs, the control input component $u_{j}(t)$ associated with the failing actuator $j$ may become an arbitrary signal which can be expressed as

$$
u_{j}(t)=\bar{u}_{j}(t), t \geq t_{j}, j \in\{1,2, \ldots, m\}
$$

Although we do not know the value of the control input when the failure occurs, we can model the failure based on some knowledge of the structure of the actuator failing signal as

$$
\bar{u}_{j}(t)=\bar{u}_{j 0}+\sum_{i=1}^{n_{j}} \bar{u}_{i j} f_{a i j}(t), t \geq t_{j}
$$


where $n_{j}$ is the number of components in the failing signal, $f_{a i j}$ are known functions corresponding to each component of the actuator, and $j, t_{j}, \bar{u}_{i j}$ are unknown failure index, failure time and failure value of each component of the actuator. With some specifications of the unknown constants $\bar{u}_{j 0}, \bar{u}_{i j}$, and known functions $f_{a i j}(t)$, we can model some common actuator failures, for example, the lock in-place failure of the actuator: $\bar{u}_{j}=\bar{u}_{j 0}$, or the complete failure of a motor in the system: $\bar{u}_{j}=0$. The terms $\bar{u}_{i j} f_{a i j}(t)$ in the actuator failure model can be used to cover additional types of time varying actuator failures.

\subsubsection{Concurrrent Actuator Failures}

An autonomous robotic system that has multiple actuators in the system such as a Hexapod often uses serial-link structures to communicate between the controller and the actuators in the system. If there is a damage in a part of the communication link, it may cause every actuator in the same chain to fail at the same time. The concurrent actuator failures, where more than one actuator fails at the same time, is a crucial problem in the robotic field.

In this study, we design an adaptive actuator failure compensation scheme for a Hexapod system. The developed adaptive control scheme can guarantee the desired closed-loop stability and asymptotic tracking, despite concurrent actuator failures. Since the Hexapod has six manipulators, the controller can compensate up to three concurrent failures before the system becomes underactuated.

\subsection{Nominal control design}

In this section, we develop a nominal controller for the system, assuming the knowledge of the actuator failures are known. Such a controller structure can be used to 
design an adaptive actuator failure compensation scheme for the system with uncertain actuator failures. Our control objective is to control the system in equation (5.6) whose actuators $u(t)$ are subject to the failures (5.11), that is, to design a feedback control signal $v(t)$ to make the system output $y(t)$ (orientation $\theta(t)$ of the platform) tracks a desirable trajectory $y_{m}(t)$ asymptotically. First, we specify a desired feedback control signal $\omega_{d}$ for $\omega=g_{2}\left(x_{1}, x_{2}\right) u$ from a backstepping method $[14,15]$ :

$$
\omega_{d}=-g_{1}\left(x_{1}, x_{2}\right)-c_{2} z_{2}-z_{1}+\dot{\beta}
$$

with the design function $\beta=-c_{1} z_{1}+\dot{y}_{m}$.

The nominal control law will guarantee that the control input signal matches the desirable signal $\omega_{d}$ when actuator failures occur.

Since the controller needs to handle multiple cases of actuator failure, we select a nominal controller structure as a combination of the nominal controller of each actuator failure case. We first design multiple individual control schemes for each actuator failure case.

Design for the no failure case. In the case of no actuator failure in the system, we have control signal $u(t)=v(t)$. We need to design $v(t)=v^{*}(t)$ such that $g_{2}\left(x_{1}, x_{2}\right) v^{*}=\omega_{d}$. We consider

$$
v^{*}(t)=v_{(1)}^{*}(t)=h_{a}\left(x_{1}, x_{2}\right) v_{a(1)}^{*}(t),
$$

where $h_{a}\left(x_{1}, x_{2}\right) \in R^{6 \times 6}$, and $v_{a(1)}^{*}(t) \in R^{6 \times 1}$ satisfy

$$
g_{2}\left(x_{1}, x_{2}\right) h_{a}\left(x_{1}, x_{2}\right) v_{a(1)}^{*}=\omega_{d} .
$$


Since the Hexapod has six manipulators to collectively move the orientation of the platform, we have some flexibilities when choosing the matrix $h_{a}\left(x_{1}, x_{2}\right)$. We can choose the matrix such that the system is optimized subject to some desirable constrains. With chosen $h_{a}\left(x_{1}, x_{2}\right)$, the intermediate control signal can be written as

$$
v_{a(1)}^{*}(t)=K_{a}\left(x_{1}, x_{2}\right) \omega_{d}
$$

where $K_{a}$ is a matrix in $R^{6 \times 3}$.

In this study, we choose to optimize the system to distribute the workload to each actuator equally. We design a controller such that the output of the adjacent actuator produces an equivalent force if possible, in this actuator failure case we choose $v_{1}=v_{2}, v_{3}=v_{4}$, and $v_{5}=v_{6}$. With this constraint, we have

$$
\begin{aligned}
v^{*}(t) & =v_{(1)}^{*}(t)=\left[v_{1}, v_{1}, v_{3}, v_{3}, v_{5}, v_{5}\right]^{T} \\
v_{b(1)}^{*}(t) & =h_{1}\left(x_{1}, x_{2}\right) v_{0(1)}^{*}(t),
\end{aligned}
$$

where $v_{0(1)}^{*}(t)=\left[v_{1}, v_{2}, v_{3}\right]$. With $g_{2 a 1}=\left[\frac{1}{2}\left(g_{21}+g_{22}\right), \frac{1}{2}\left(g_{23}+g_{24}\right), \frac{1}{2}\left(g_{25}+g_{26}\right)\right]$ we can choose a new matrix $h_{1}\left(x_{1}, x_{2}\right) \in R^{3 \times 3}$ such that $g_{2 a}\left(x_{1}, x_{2}\right) h_{1}\left(x_{1}, x_{2}\right)$ is invertible, so $v_{0(1)}^{*}(t) \in R^{3 \times 1}$ can be chosen as

$$
v_{0(1)}^{*}(t)=K_{1}\left(x_{1}, x_{2}\right) \omega_{d}
$$

where $K_{1}=\left(g_{2 a 1} h_{1}\left(x_{1}, x_{2}\right)\right)^{-1}$.

Design for one actuator failure case. There are six possible configurations of actuator failure with one failing actuator. Since the Hexapod has a relatively symmetric structure, the controller for each possible failure configuration can be designed similarly for each configuration, so we will only choose actuator $u_{1}$ failure case to 
serve as an example in this study. In case of actuator $u_{1}$ failing, we have the signal $u_{1}=\bar{u}_{1}$. The nominal control signal $v(t)=v^{*}(t)$ can be chosen as

$$
v^{*}(t)=v_{(2)}^{*}(t)=\left[0, v_{2}^{*}, v_{3}^{*}, v_{3}^{*}, v_{5}^{*}, v_{5}^{*}\right]^{T}
$$

With $v_{a(2)}^{*}(t)=\left[v_{2}^{*}, v_{3}^{*}, v_{5}^{*}\right]$ we have

$$
v_{a(2)}^{*}(t)=h_{2}\left(x_{1}, x_{2}\right) v_{0(2)}^{*}(t)
$$

where the matrix $h_{2}\left(x_{1}, x_{2}\right) \in R^{3 \times 3}$. The signal $v_{0(2)}^{*}(t) \in R^{3 \times 1}$ can be chosen to satisfy

$$
g_{21}\left(x_{1}, x_{2}\right) \bar{u}_{1}+g_{2 a 2} h_{2}\left(x_{1}, x_{2}\right) v_{0(2)}^{*}(t)=\omega_{d}
$$

where $g_{2 a 2}=\left[g_{22}, \frac{1}{2}\left(g_{23}+g_{24}\right), \frac{1}{2}\left(g_{25}+g_{26}\right)\right]$. The signal $v_{0(2)}^{*}(t)$ can be written as

$$
v_{0(2)}^{*}(t)=K_{21}\left(x_{1}, x_{2}\right) \omega_{d}+K_{22}\left(x_{1}, x_{2}\right) \bar{u}_{1}
$$

where $K_{21}=\left(g_{2 a 2} h_{2}\left(x_{1}, x_{2}\right)\right)^{-1}$ and $K_{22}=-\left(g_{2 a 2} h_{2}\left(x_{1}, x_{2}\right)\right)^{-1} g_{21}\left(x_{1}, x_{2}\right)$.

Design for two actuator failure case. In this case, we consider both actuator $u_{1}$ and $u_{2}$ fail at the same time. When actuator $u_{1}$ and $u_{2}$ failure, we have the signals $u_{1}=\bar{u}_{1}$ and $u_{2}=\bar{u}_{2}$. The nominal control signal $v(t)=v^{*}(t)$ can be chosen as

$$
v^{*}(t)=v_{(3)}^{*}(t)=\left[0,0, v_{3}^{*}, v_{4}^{*}, v_{4}^{*}, v_{6}^{*}\right]^{T} .
$$

With $v_{a(3)}^{*}(t)=\left[v_{3}^{*}, v_{4}^{*}, v_{6}^{*}\right]$ we have

$$
v_{a(3)}^{*}(t)=h_{3}\left(x_{1}, x_{2}\right) v_{0(3)}^{*}(t),
$$


where the matrix $h_{3}\left(x_{1}, x_{2}\right) \in R^{3 \times 3}$. The signal $v_{0(3)}^{*}(t) \in R^{3 \times 1}$ can be chosen to satisfy

$$
g_{21} \bar{u}_{1}+g_{22} \bar{u}_{2}+g_{2 a 3} h_{2} v_{0(2)}^{*}(t)=\omega_{d}
$$

where $g_{2 a 3}=\left[g_{23}, \frac{1}{2}\left(g_{24}+g_{25}\right), g_{26}\right]$. The signal $v_{0(3)}^{*}(t)$ can be written as

$$
v_{0(3)}^{*}(t)=K_{31} \omega_{d}+K_{32} \bar{u}_{1}+K_{33} \bar{u}_{2},
$$

where $K_{31}=\left(g_{2 a 3} h_{3}\left(x_{1}, x_{2}\right)\right)^{-1}$,

$$
\begin{aligned}
& K_{32}=-\left(g_{2 a 3} h_{3}\left(x_{1}, x_{2}\right)\right)^{-1} g_{21}\left(x_{1}, x_{2}\right) \text { and } \\
& K_{33}=-\left(g_{2 a 3} h_{3}\left(x_{1}, x_{2}\right)\right)^{-1} g_{22}\left(x_{1}, x_{2}\right) .
\end{aligned}
$$

Design for three actuator failure case. In this case, we choose actuator $u_{1}, u_{3}$ and $u_{5}$ fail. When actuator $u_{1}, u_{3}$ and $u_{2}$ failure, we have the signals $u_{1}=\bar{u}_{1}, u_{3}=\bar{u}_{3}$ and $u_{5}=\bar{u}_{5}$. The nominal control signal $v(t)=v^{*}(t)$ can be chosen as

$$
v^{*}(t)=v_{(4)}^{*}(t)=\left[0, v_{2}^{*}, 0, v_{4}^{*}, 0, v_{6}^{*}\right]^{T}
$$

With $v_{a(4)}^{*}(t)=\left[v_{2}^{*}, v_{4}^{*}, v_{6}^{*}\right]$ we have

$$
v_{a(4)}^{*}(t)=h_{4}\left(x_{1}, x_{2}\right) v_{0(4)}^{*}(t)
$$

where the matrix $h_{4}\left(x_{1}, x_{2}\right) \in R^{3 \times 3}$ and the signal $v_{0(4)}^{*}(t) \in R^{3 \times 1}$ can be chosen to satisfy

$$
g_{21} \bar{u}_{1}+g_{23} \bar{u}_{3}+g_{25} \bar{u}_{5}+g_{2 a 4} h_{4} v_{0(4)}^{*}(t)=\omega_{d}
$$


where $g_{2 a 4}=\left[g_{22}, g_{24}, g_{26}\right]$. The signal $v_{0(4)}^{*}(t)$ can be written as

$$
v_{0(4)}^{*}(t)=K_{41} \omega_{d}+K_{42} \bar{u}_{1}+K_{43} \bar{u}_{3}+K_{44} \bar{u}_{5},
$$

where $K_{41}=\left(g_{2 a 4} h_{4}\left(x_{1}, x_{2}\right)\right)^{-1}$,

$$
\begin{aligned}
& K_{42}=-\left(g_{2 a 4} h_{4}\left(x_{1}, x_{2}\right)\right)^{-1} g_{21}\left(x_{1}, x_{2}\right), \\
& K_{43}=-\left(g_{2 a 4} h_{4}\left(x_{1}, x_{2}\right)\right)^{-1} g_{23}\left(x_{1}, x_{2}\right) \text { and } \\
& K_{44}=-\left(g_{2 a 4} h_{4}\left(x_{1}, x_{2}\right)\right)^{-1} g_{25}\left(x_{1}, x_{2}\right) .
\end{aligned}
$$

Composite control design. With the control signals for all cases, we design a composite nominal control law for $v(t)=v^{*}(t)$ as

$$
v^{*}(t)=\chi_{1}^{*} v_{(1)}^{*}(t)+\chi_{2}^{*} v_{(2)}^{*}(t)+\chi_{3}^{*} v_{(3)}^{*}(t)+\chi_{4}^{*} v_{(4)}^{*}(t)
$$

where $\chi_{1}^{*}, \chi_{2}^{*}, \chi_{3}^{*}, \chi_{4}^{*}$ are the indicator functions of the actuator failure: $\chi_{i}^{*}=1$ for $i=1,2,3,4$ if the associate failure pattern occurs, $\chi_{i}^{*}=0$ otherwise. The signal $v_{(1)}^{*}(t)$ is the nominal control signal in case of no actuator failure in the system, $v_{(2)}^{*}(t)$ is the nominal control signal for single actuator failure case (actuator $u_{1}$ fails), $v_{(3)}^{*}(t)$ is the nominal control signal for the two actuator failure case (actuators $u_{1}$ and $u_{2}$ fail) and $v_{(4)}^{*}(t)$ is the nominal control signal for the three actuator failure case (actuators $u_{1}, u_{3}, u_{5}$ fail). The other possible cases of actuator failure can be designed and added to the composite control in a similar way with the example cases. Because of the indicator functions and the nominal control signals, the composite design ensures $g_{2}\left(x_{1}, x_{2}\right) u=\omega_{d}$ for every case of failure that we consider. 


\subsection{Adaptive Failure Compensation Design}

The nominal control design, which is developed in Section IV, needs information of the actuator failures. In this section, we develop an adaptive actuator failure compensation scheme, which can achieve the control objective in the presence of uncertain actuator failures without the knowledge of the failing actuators and its signals.

\subsubsection{Adaptive Controller Structure}

The adaptive control algorithm is developed based on the nominal controller structure. Since we do not know the values of $\chi_{1}^{*}, \chi_{2}^{*}, \chi_{3}^{*}, \chi_{4}^{*}$ and the value of failing actuator $\bar{u}$ we first design the adaptive controller structure as

$$
v(t)=v_{\chi(1)}(t)+v_{\chi(2)}(t)+v_{\chi(3)}(t)+v_{\chi(4)}(t),
$$

where $v_{\chi(j)}(t)$ are the estimates of $\chi_{j}^{*} v_{(i)}^{*}$ for $j=1,2,3,4$.

We estimate $\chi_{j}^{*}$ with $\operatorname{diag}\left\{\chi_{j 1}, \chi_{j 2}, \chi_{j 3}\right\}$, where we estimate the parameter $\chi_{j}^{*}$ three times for achieving a suitable parameterization. The actuator failure $\bar{u}_{i}(t)$ can be parametrized using the actuator failure model (5.11).

In order to illustrate this concept, we use the parameterization of $v_{\chi(3)}(t)$ as an example in our study.

With $v_{\chi(3)}(t)=\left[0,0, v_{\chi(3) 3}, v_{\chi(3) 4}, v_{\chi(3) 4}, v_{\chi(3) 6}\right]^{T}$ and $\bar{u}_{i}$ are the error signals, let $v_{\chi a(3)}(t)=\left[v_{\chi(3) 3}, v_{\chi(3) 4}, v_{\chi(3) 6}\right]^{T}$ we can derive $v_{\chi a(3)}(t)$ from the nominal version 
$v_{a(3)}(t)^{*}$ as

$$
\chi_{3}^{*} v_{a(3)}^{*}(t)=\chi_{3}^{*} h_{3} K_{31} \omega_{d}+\chi_{3}^{*} \bar{u}_{1} h_{3} K_{32}+\chi_{3}^{*} \bar{u}_{2} h_{3} K_{33} .
$$

Then, the estimate of $\chi_{3}^{*} v_{a(3)}^{*}(t)$ can be written as

$$
\begin{gathered}
v_{\chi a(3)}(t)=\operatorname{diag}\left\{\chi_{31}, \chi_{32}, \chi_{33}\right\} h_{3} K_{31} \omega_{d} \\
+\left[\begin{array}{c}
\rho_{31(1)}^{T} f_{a 1}(t) \phi_{311} \\
\rho_{31(2)}^{T} f_{a 1}(t) \phi_{312} \\
\rho_{31(3)}^{T} f_{a 1}(t) \phi_{313},
\end{array}\right]+\left[\begin{array}{c}
\rho_{32(1)}^{T} f_{a 2}(t) \phi_{321} \\
\rho_{32(2)}^{T} f_{a 2}(t) \phi_{322} \\
\rho_{33(3)}^{T} f_{a 2}(t) \phi_{323},
\end{array}\right],
\end{gathered}
$$

where $\operatorname{diag}\left\{\chi_{31}, \chi_{32}, \chi_{33}\right\}$ is the estimate of $\operatorname{diag}\left\{\chi_{31}^{*}, \chi_{32}^{*}, \chi_{32}^{*}\right\}$ with $\chi_{3}^{*}=\chi_{31}^{*}=\chi_{32}^{*}=$ $\chi_{33}^{*}$. The terms $\chi_{31}^{*} \rho_{3 i}^{*}, \chi_{32}^{*} \rho_{3 i}^{*}$ and $\chi_{33}^{*} \rho_{3 i}^{*}$ are estimated by $\rho_{3 i(1)}, \rho_{3 i(2)}$ and $\rho_{3 i(3)}$, for $i=1,2$ with $\phi_{31}=\left[\phi_{311}, \phi_{312}, \phi_{313}\right]^{T}=h_{3} K_{32}$ and $\phi_{32}=\left[\phi_{321}, \phi_{322}, \phi_{323}\right]^{T}=h_{3} K_{33}$.

The parametrization of $v_{\chi(1)}(t), v_{\chi(2)}(t)$ and $v_{\chi(4)}(t)$ can be done in the similar way as $v_{\chi(3)}(t)$. With this parametrized controller structure, we will develop adaptive laws to update the parameters $\operatorname{diag}\left\{\chi_{i 1}, \chi_{i 2}, \chi_{i 3}\right\}$, for $i=1,2,3,4, \rho_{2(j)}, \rho_{31(j)}, \rho_{32(j)}, \rho_{41(j)}, \rho_{42(j)}$, and $\rho_{43(j)}$, for $j=1,2,3$, so that the system can achieve the control objective in the presence of uncertain actuator failure.

\subsubsection{Adaptive Laws}

In this subsection, we develop an adaptive scheme for updating the parameters of the controller (5.32). The adaptive laws for $\chi_{i j}$ with $i=1,2,3,4$ and $j=1,2,3$ are chosen as

$$
\dot{\chi}_{i j}=-\gamma_{i j} z_{2}^{T} g_{2 a i j} \mu_{1 j}+\varrho_{\chi_{i j}}
$$


where $\gamma_{i j}>0$, are the adaptation gains, and

$$
\mu_{i}=\left[\mu_{i 1}, \mu_{i 2}, \mu_{i 3}\right]^{T}=h_{i} K_{i 1} \omega_{d}
$$

with $h_{1}, h_{2}, h_{3}, h_{4}, K_{1}, K_{21}, K_{31}$, and $K_{41}$ being the design matrices from the adaptive controller structure (5.32).

The projection function $\varrho_{\chi i j}$ is chosen as

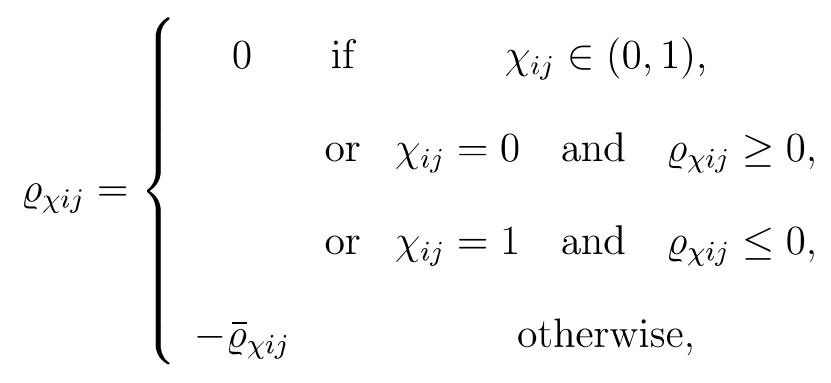

with $\bar{\varrho}_{\chi i j}$ defined as $\bar{\varrho}_{\chi i j}=-\gamma_{i j} z_{2}^{T} g_{a 2 i j} \mu_{1 j}$.

Similarly, the adaptive laws for $\rho_{i(j)}=\left[\rho_{i(j)(1)}, \ldots, \rho_{i(j)\left(n_{i}\right)}\right]^{T}$ become

$$
\begin{aligned}
& \dot{\rho}_{2(j)}=-\Gamma_{(2 j)} f_{a j} z_{2}^{T} g_{2 a 2 j} \phi_{2 j}+\varrho_{\rho_{2(j)}}, \quad \dot{\rho}_{31(j)}=-\Gamma_{(31)} f_{a j} z_{2}^{T} g_{2 a 3 j} \phi_{31(j)}+\varrho_{\rho_{31(j)}} \\
& \dot{\rho}_{32(j)}=-\Gamma_{(32)} f_{a j} z_{2}^{T} g_{2 a 3 j} \phi_{32(j)}+\varrho_{\rho_{32(j)}}, \quad \dot{\rho}_{41(j)}=-\Gamma_{(41)} f_{a j} z_{2}^{T} g_{2 a 4 j} \phi_{41(j)}+\varrho_{\rho_{41(j)}} \\
& \dot{\rho}_{42(j)}=-\Gamma_{(42)} f_{a j} z_{2}^{T} g_{2 a 4 j} \phi_{42(j)}+\varrho_{\rho_{42(j)}}, \quad \dot{\rho}_{43(j)}=-\Gamma_{(43)} f_{a j} z_{2}^{T} g_{2 a 4 j} \phi_{43(j)}+\varrho_{\rho_{43(j)}},
\end{aligned}
$$

where the projection function $\varrho_{\rho i(j)}=\left[\varrho_{\rho i(j)(1)}, \ldots, \varrho_{\rho i(j)\left(n_{i}\right)}\right]^{T}$ is chosen as

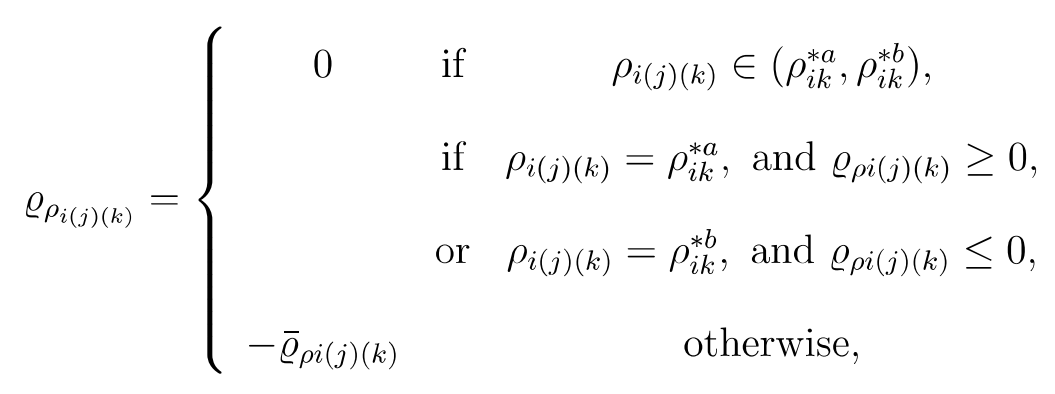


with $\bar{\varrho}_{\rho i(j)}=\left[\bar{\varrho}_{\rho i(j)(1)}, \ldots, \bar{\varrho}_{\rho i(j)\left(n_{i}\right)}\right]^{T}$ defined as

$$
\begin{aligned}
& \dot{\rho}_{2(j)}=-\Gamma_{(2 j)} f_{a j} z_{2}^{T} g_{2 a 2 j} \phi_{2 j}, \quad \dot{\rho}_{31(j)}=-\Gamma_{(31)} f_{a j} z_{2}^{T} g_{2 a 3 j} \phi_{31(j)} \\
& \dot{\rho}_{32(j)}=-\Gamma_{(32)} f_{a j} z_{2}^{T} g_{2 a 3 j} \phi_{32(j)}, \quad \dot{\rho}_{41(j)}=-\Gamma_{(41)} f_{a j} z_{2}^{T} g_{2 a 4 j} \phi_{41(j)} \\
& \dot{\rho}_{42(j)}=-\Gamma_{(42)} f_{a j} z_{2}^{T} g_{2 a 4 j} \phi_{42(j)}, \quad \dot{\rho}_{43(j)}=-\Gamma_{(43)} f_{a j} z_{2}^{T} g_{2 a 4 j} \phi_{43(j)},
\end{aligned}
$$

where $\Gamma_{(i j)}=\Gamma_{(i j)}^{T}>0$ are the adaptation gain matrices.

Here we use a parameter projection scheme to ensure the boundedness of the estimated parameters in the presence of actuator failure uncertainties.

We have the range of $\chi_{i}^{*}$ as $0 \leq \chi_{i}^{*} \leq 1$. Since we know $\chi_{i}^{*}$ is between 0 and 1 , we can use the parameter projection to ensure their estimates $\chi_{i j}$ are in the interval $[0,1]$, as continuous parameters.

Let $\rho_{i k}^{* a}, \rho_{i k}^{* b}$ be the upper and lower bounds of the components $\rho_{i k}^{*}$ of $\rho_{i}^{*}, i=$ $2,31,32,41,42,43 k=1,2, \ldots, k_{n}$, that is, $\rho_{i k}^{* a} \leq \rho_{i k}^{*} \leq \rho_{i k}^{* b}$. The upper and lower bounds $\rho_{i k}^{* a}, \rho_{i k}^{* b}$ can be obtained from the maximum and minimum forces that each actuator can produce.

With the parameter projection scheme and chosen initial conditions $0 \leq \chi_{i j}(0) \leq$ $1, \rho_{i k}^{* a} \leq \rho_{i(j k)}(0) \leq \rho_{i k}^{* b}$, we can guarantee the boundedness of every estimated parameter used in the adaptive control scheme.

Theorem 1. The adaptive actuator failure compensation scheme with the feedback control law (5.32) whose parameters are updated by the adaptive laws (5.35)-(5.40), when applied to the Hexapod model (5.6) subject to actuator failures (5.11) whose failure pattern, value and time instants are uncertain, guarantees the closed-loop signal boundedness and asymptotic output tracking: $\lim _{t \rightarrow \infty}\left(y(t)-y_{m}(t)\right)=0$. 
Proof. The proof is similar to that in [10] for a nonlinear system and is refined here. We first consider the error signals $z_{1}=x_{1}-y_{m}$, and $z_{2}=x_{2}-\beta$. With uncertain actuator failures, we have the derivatives of $z_{1}$ and $z_{2}$ as

$$
\dot{z}_{1}=-c_{1} z_{1}+z_{2}, \dot{z}_{2}=-z_{1}-c_{2} z_{2}+g_{2}\left(x_{1}, x_{2}\right)(I-\sigma)\left(v-v^{*}\right) .
$$

Because the derivative of the error signal $\dot{z}_{2}$ contains the actuator failure pattern $\sigma$ which changes according to each actuator failure case, we need to consider a new Lyapunov function candidates for each actuator failure pattern to ensure the system stability.

For the case of no failure, we consider the following Lyapunov function candidate

$$
\begin{aligned}
V_{1}= & \frac{1}{2} z_{1}^{T} z_{1}+\frac{1}{2} z_{2}^{T} z_{2}+\frac{1}{2} \sum_{i=1}^{3} \sum_{j=1}^{3} \tilde{\chi}_{i j}^{2} \gamma_{i j}^{-1} \\
& +\frac{1}{2} \sum_{k} \sum_{j=1}^{3} \tilde{\rho}_{k(j)}^{T} \Gamma_{k j}^{-1} \tilde{\rho}_{k(j)},
\end{aligned}
$$

for $k=2,31,32,41,42,43$.

With the parameter projection scheme, we have the derivative of each term as

$$
\begin{aligned}
\frac{d}{d t} & \frac{1}{2}\left(z_{1}^{T} z_{1}+z_{2}^{T} z_{2}\right)=-c_{1} z_{1}^{T} z_{1}-c_{2} z_{2}^{T} z_{2}+z_{2}^{T} g_{2}\left(x_{1}, x_{2}\right)(I-\sigma) \tilde{v}_{\chi(1)} \\
& \left.+z_{2}^{T} g_{2}\left(x_{1}, x_{2}\right)(I-\sigma)\left[0, \tilde{v}_{\chi a(2)}^{T}(t)\right]^{T}+z_{2}^{T} g_{2}\left(x_{1}, x_{2}\right)(I-\sigma)\left[\tilde{v}_{\chi(31)}(t), 0, \tilde{v}_{\chi(33)}((t))^{4}\right] \mathbb{A}^{\mathrm{T}} 3\right) \\
\frac{d}{d t}( & \left.\frac{1}{2} \sum_{i=1}^{3} \tilde{\chi}_{1 i}^{2} \gamma_{1 i}^{-1}\right)=-z_{2}^{T}\left[\sum_{i=1}^{3} \tilde{\chi}_{1 i} g_{2 i} \mu_{1 i}\right]+\sum_{i=1}^{3} \tilde{\chi}_{1 i} \gamma_{1 i}^{-1} \varrho_{\chi_{1 i}} \leq-z_{2}^{T} g_{2}\left(x_{1}, x_{2}\right) \tilde{v}_{\chi(1)} \\
\frac{d}{d t} & \left(\frac{1}{2}\left(\sum_{i=1}^{3} \tilde{\rho}_{1(i)}^{T} \Gamma_{1 i}^{-1} \tilde{\rho}_{1(i)}\right)\right)=\sum_{i=1}^{3} \tilde{\chi}_{2 i} \gamma_{2 i}^{-1} \varrho_{\chi 2 i}+\sum_{i=1}^{2} \tilde{\rho}_{1(i)}^{T} \Gamma_{1 i}^{-1} \varrho_{\rho_{1(i)}} \leq 0
\end{aligned}
$$


Here we have used the parameter projection property and ; that is,

$$
\tilde{\chi}_{i j} \gamma_{i j}^{-1} \varrho_{\chi_{i j}} \leq 0 \quad \tilde{\rho}_{i(j)}^{T} \Gamma_{i j}^{-1} \varrho_{\rho_{i(j)}} \leq 0 .
$$

With $\sigma=\operatorname{diag}\{0,0,0\}$, the derivative of the Lyapunov function (5.42) becomes

$$
\dot{V}_{a 1} \leq-c_{1} z_{1}^{T} z_{1}-c_{2} z_{2}^{T} z_{2} \leq 0 .
$$

For the actuator $u_{1}$ failure case, we have the actuator failure pattern $\sigma=\operatorname{diag}\{1,0,0\}$. We need a new Lyapunov function candidate $V_{a 2}$ as

$$
\begin{aligned}
V_{a 2} & =\frac{1}{2} z_{1}^{T} z_{1}+\frac{1}{2} z_{2}^{T} z_{2}+\frac{1}{2}\left[\sum_{i=2}^{3} \tilde{\chi}_{1 i}^{2} \gamma_{1 i}^{-1}+\sum_{i=1}^{2} \tilde{\chi}_{2 i}^{2} \gamma_{2 i}^{-1}+\tilde{\chi}_{32}^{2} \gamma_{32}^{-1}+\sum_{i=1}^{2} \tilde{\rho}_{1(i)}^{T} \Gamma_{1 i}^{-1} \tilde{\rho}_{1(i)}\right. \\
& \left.+\tilde{\rho}_{2(2)}^{T} \Gamma_{22}^{-1} \tilde{\rho}_{2(2)}\right] .
\end{aligned}
$$

To obtain the derivatives of the Lyapunov function candidates in (5.48), we consider the derivative of each term similar to the derivation in the case of no actuator failure. In this case, we have

$$
\begin{aligned}
\frac{d}{d t}( & \left.\frac{1}{2} \sum_{i=2}^{3} \tilde{\chi}_{1 i}^{2} \gamma_{1 i}^{-1}\right)=-z_{2}^{T} g_{2}\left(x_{1}, x_{2}\right)\left[0, \tilde{v}_{\chi a(2)}^{T}(t)\right]^{T}+\sum_{i=2}^{3} \tilde{\chi}_{2 i} \gamma_{2 i}^{-1} \varrho_{\chi 2 i} \\
& \leq-z_{2}^{T} g_{2}\left(x_{1}, x_{2}\right)(I-\sigma) \tilde{v}_{\chi(1)} \\
\frac{d}{d t}( & \left.\frac{1}{2}\left(\sum_{i=1}^{2} \tilde{\chi}_{2 i}^{2} \gamma_{2 i}^{-1}+\sum_{i=1}^{2} \tilde{\rho}_{1(i)}^{T} \Gamma_{1 i}^{-1} \tilde{\rho}_{1(i)}\right)\right) \\
& =-z_{2}^{T} g_{2}\left(x_{1}, x_{2}\right)\left[0, \tilde{v}_{\chi a(2)}^{T}(t)\right]^{T}+\sum_{i=1}^{2} \tilde{\chi}_{2 i} \gamma_{2 i}^{-1} \varrho_{\chi 2 i}+\sum_{i=1}^{2} \tilde{\rho}_{1(i)}^{T} \Gamma_{1 i}^{-1} \varrho_{\rho_{1(i)}} \\
& \leq-z_{2}^{T} g_{2}\left(x_{1}, x_{2}\right)(I-\sigma)\left[0, \tilde{v}_{\chi a(2)}^{T}(t)\right]^{T}
\end{aligned}
$$




$$
\begin{aligned}
\frac{d}{d t}( & \left.\frac{1}{2}\left(\tilde{\chi}_{32}^{2} \gamma_{32}^{-1}+\tilde{\rho}_{2(2)} \Gamma_{22}^{-1} \tilde{\rho}_{2(2)}^{T}\right)\right) \\
& =-z_{2}^{T} g_{2}\left(x_{1}, x_{2}\right)\left[0, \tilde{v}_{\chi a(2)}^{T}(t)\right]^{T}+\tilde{\chi}_{32} \gamma_{32}^{-1} \varrho_{\chi 32}+\tilde{\rho}_{2(2)}^{T} \Gamma_{22}^{-1} \varrho_{\rho_{2(2)}} \\
& \leq-z_{2}^{T} g_{2}\left(x_{1}, x_{2}\right)(I-\sigma)\left[\tilde{v}_{\chi(31)}(t), 0, \tilde{v}_{\chi(33)}(t)\right]^{T} .
\end{aligned}
$$

We notice that the terms $\tilde{\chi}_{11}^{2} \gamma_{11}^{-1}, \tilde{\chi}_{31}^{2} \gamma_{31}^{-1}$, and $\tilde{\rho}_{2(1)} \Gamma_{21}^{-1} \tilde{\rho}_{2(1)}$ disappear because $(I-\sigma)=$ $\operatorname{diag}\{0,1,1\}$, with $\sigma=\operatorname{diag}\{1,0,0\}$. We can see that the terms in the derivatives of Lyapunov function candidates (5.49)-(5.52) cancel the non-negative semidefinite parts in (5.43), and the derivatives of the Lyapunov function (5.48) becomes

$$
\dot{V}_{a 2} \leq-c_{1} z_{1}^{T} z_{1}-c_{2} z_{2}^{T} z_{2} \leq 0 .
$$

For the actuator $u_{2}$ failure case, we have the actuator failure pattern $\sigma=\operatorname{diag}\{0,1,0\}$. We use a new Lyapunov function candidate

$$
\begin{aligned}
& V_{a 3}=\frac{1}{2} z_{1}^{T} z_{1}+\frac{1}{2} z_{2}^{T} z_{2}+\frac{1}{2}\left[\sum_{i=1,3} \tilde{\chi}_{1 i}^{2} \gamma_{1 i}^{-1}+\tilde{\chi}_{22}^{2} \gamma_{22}^{-1}\right. \\
& \left.+\sum_{i=1}^{2} \tilde{\chi}_{3 i}^{2} \gamma_{3 i}^{-1}+\tilde{\rho}_{1(2)}^{T} \Gamma_{12}^{-1} \tilde{\rho}_{1(2)}+\sum_{i=1}^{2} \tilde{\rho}_{2(i)}^{T} \Gamma_{2 i}^{-1} \tilde{\rho}_{2(i)}\right] .
\end{aligned}
$$

With a similar approach as actuator $u_{1}$ failure case, we can see that the terms $\tilde{\chi}_{12}^{2} \gamma_{12}^{-1}$, $\tilde{\chi}_{21}^{2} \gamma_{21}^{-1}$, and $\tilde{\rho}_{1(1)} \Gamma_{11}^{-1} \tilde{\rho}_{1(1)}$ disappear because $(I-\sigma)=\operatorname{diag}\{1,0,1\}$. In this case, we have

$$
\dot{V}_{1} \leq-c_{1} z_{1}^{T} z_{1}-c_{2} z_{2}^{T} z_{2} \leq 0 .
$$

For the remaining actuator failure cases, we have the different actuator failure pattern $\sigma$, thus, a new Lyapunov function candidate is needed to analyze the stability of the system. We can notice that the previous Lyapunov function no longer works in the 
other actuator failure cases because we cannot guarantee that term associated with the estimated parameter corresponding to the failing actuator $\tilde{\chi}_{i j}^{2} \gamma_{i j}^{-1}$ and $\tilde{\rho}_{i(j)} \Gamma_{i j}^{-1} \tilde{\rho}_{i(j)}$ will be negative definite. Thus, the new Lyapunov function candidate can be constructed by removing such term from the original Lyapunov function $V_{1}$.

It can be proven that the derivative of the new Lyapunov functions candidate constructed this way are negative semidefinite. With the parameter projection schemes, we can show that all signals in the system are bounded. Since $z_{1}(t), z_{2}(t), \dot{z}_{1}(t) \in$ $L^{\infty} \bigcap L^{2}$, based on the Barbalat lemma, we can conclude that $\lim _{t \rightarrow \infty} z_{1}=0 . \nabla \nabla \nabla$

\subsection{Simulations Study}

In this section, we simulate the actuator failure compensation control scheme based on the Hexapod model subject to uncertain actuator failures.

\subsubsection{System Model and Simulation Conditions}

The simulations are performed based on the Hexapod model (5.6). We assign the parameters in the system based on the Stewart platform [8,9].

The base point of each manipulator is $\chi_{q i}=\left[0.30 \cos \frac{i \pi}{3}, 0.30 \cos \frac{i \pi}{3}, 0\right]$ for $i=$ $1,2, \ldots, 6$, the platform points are assigned as $p_{i}=\left[0.15 \cos \frac{i \pi}{3}, 0.15 \cos \frac{i \pi}{3}, 0\right]$, the directional vectors $d=[0.81,0.23,0.95,1,0.71,0.95 ; 0.27,0.92,0.29,0,0.7,0.29$; $0,0.31,0.1,0,0,0.1]$

The mass of each manipulator is assigned as $3.0 \mathrm{~kg}$, the mass of the platform with the payload $M_{p}=40.0 \mathrm{~kg}$ with the inertia $I_{p}=[0.05,0.003,0.004 ; 0.003,0.04,0.003$; 0.004, 0.003, 0.1]. For simplicity the spring and damping constants are chosen to be one. The initial conditions are $y(0)=[1,1,1]^{T}$, and estimated parameters have zero 
initial condition.

In this study, we set the desirable trajectory of each simulation as $y_{m}=[5 \sin (0.2 t), 5 \sin (0.2 t), 5 \sin (0.2 t)]^{T}$, we consider three cases of actuator failures as follows:

\section{One actuator failure case:}

(i) No actuator failure case: $u(t)=v(t)$ for $0 \leq t<50 s$,

(ii) Actuator $u_{1}$ failure case: $u_{1}=\bar{u}_{1}=20 \sin (0.1 t)$ at time $50 \leq t<100 s$.

\section{Two actuator failure case:}

(i) No actuator failure case: $u(t)=v(t)$ for $0 \leq t<50 \mathrm{~s}$,

(ii) Actuator $u_{1}$ and $u_{2}$ failure case: $u_{1}=\bar{u}_{1}=20 \sin (0.1 t), u_{2}=\bar{u}_{2}=10 \sin (0.1 t)$ at $50 \leq t<100 s$

\section{Three actuator failure case:}

(i) No actuator failure case: $u(t)=v(t)$ for $0 \leq t<50 \mathrm{~s}$,

(ii) Actuator $u_{1}, u_{3}$, and $u_{5}$ failure case: $u_{1}=\bar{u}_{1}=20 \sin (0.1 t), u_{3}=\bar{u}_{3}=$ $10 \sin (0.1 t), u_{5}=\bar{u}_{5}=10 \sin (0.1 t)$ at $50 \leq t<100 s$.

\subsubsection{Simulation Results}

For each actuator failure case, the simulation results show the system output $\theta(t)=$ $\left[\theta_{x}, \theta_{y}, \theta_{z}\right]^{T}$ in Figures $5.2-5.4$. 

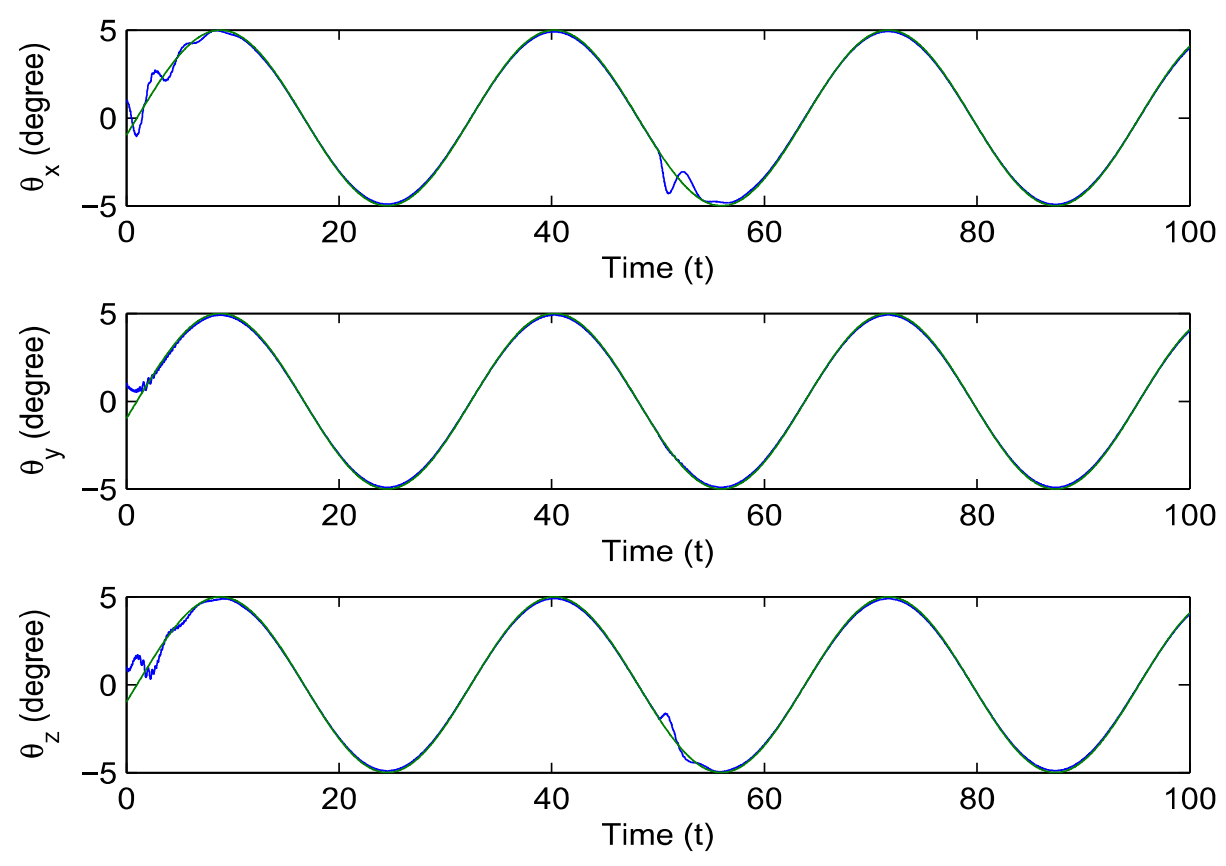

Figure 5.2: System output $\theta(t)$ vs. $y_{m}(t)$ for one actuator failure case.
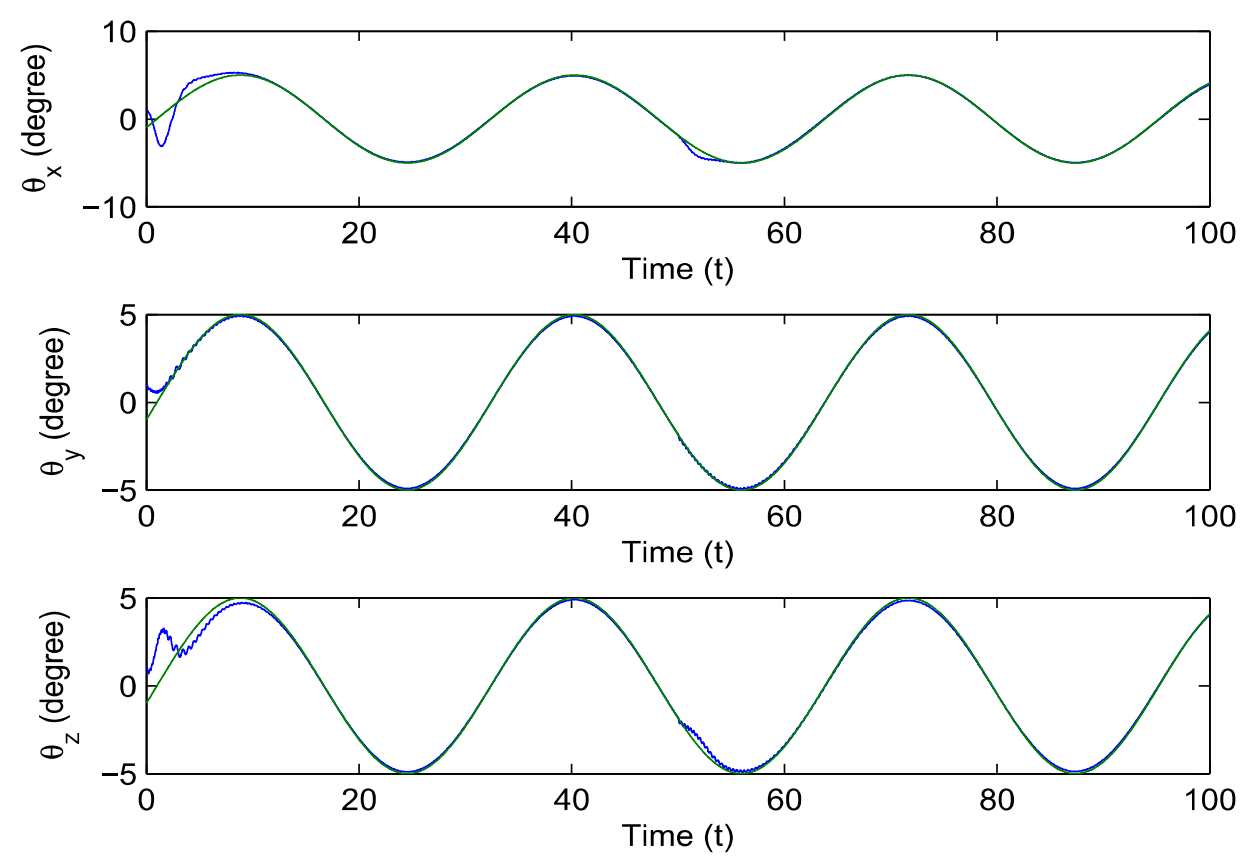

Figure 5.3: System output $\theta(t)$ vs. $y_{m}(t)$ for two actuator failure case. 

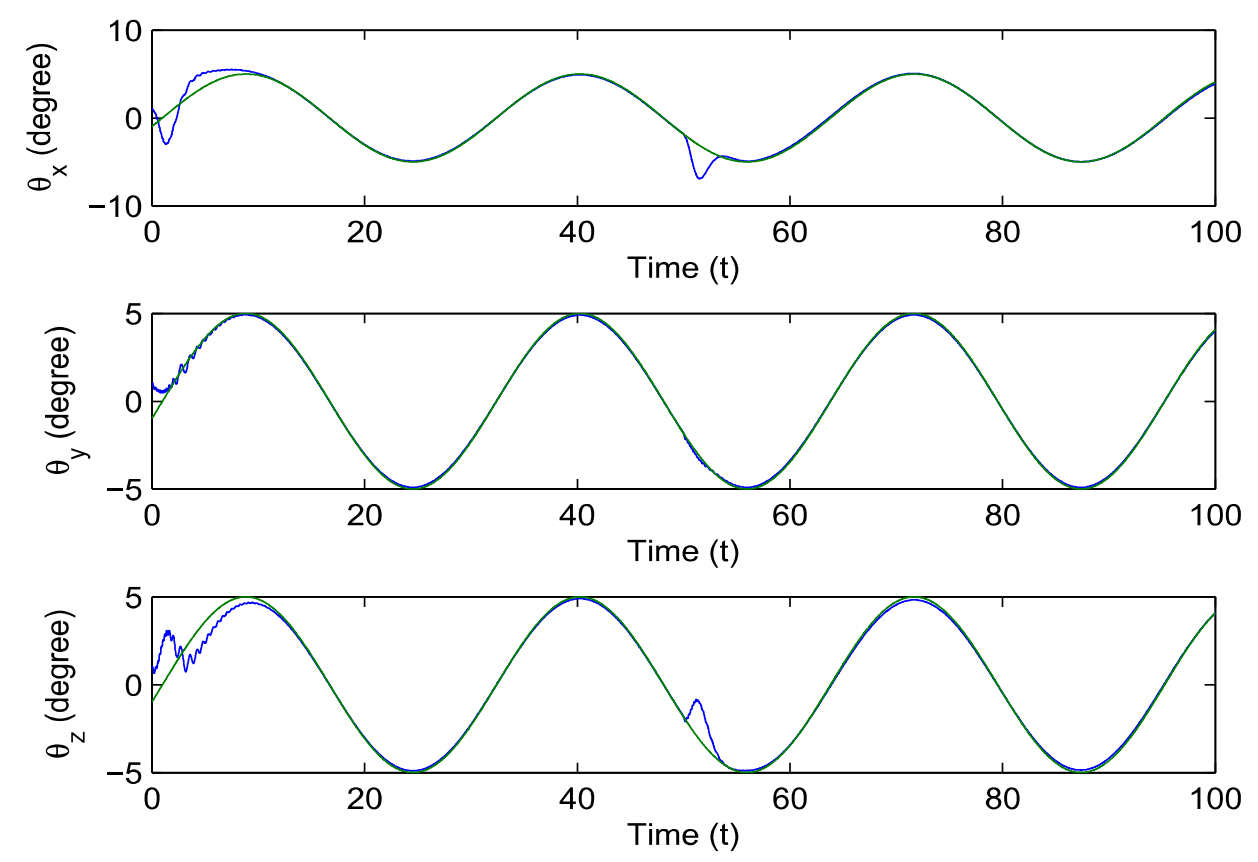

Figure 5.4: System output $\theta(t)$ vs. $y_{m}(t)$ for three actuator failure case.

The simulation results confirm that the adaptive actuator failure compensation scheme can guarantee that the tracking error of the system goes to zero for a sinusoidal desirable trajectory. The adaptive actuator failure compensation scheme allows up to three actuators to fail at the same time.

\subsection{Conclusions}

A Hexapod plays an important part in many projects. Developing an adaptive actuator failure compensation scheme that can effectively compensate for the actuator failures in the system is important for those applications and is a challenging control problem. Since the system has six manipulators, we can design a controller, which utilizes the redundancy to control a Hexapod with concurrent failures.

In this chapter, we have studied the Hexapod. Our research shows that, with a complete parametization of failure patterns, a desirable closed-loop stability and 
asymptotic tracking of the system can be achieved, despite the concurrent actuator failures. The simulation results also verified the performance of the adaptive control algorithm when applied to the Hexapod model subject to uncertain actuator failures. 


\section{Chapter 6}

\section{Design for A Cooperative System with Parameter Uncertainties}

One of the most important characteristic of robot manipulators, which make a manipulator difficult to be controlled, is that the dynamic of the manipulators is highly nonlinear. Although it is possible to design a controller to achieve a good performance if we known a precise model of the system [15], the controller is not capable of handling any changes and uncertainties in the system. In general, a robot manipulator often interacts with environments such as grabbing or carrying different objects with unknown dimensions, orientations or gripping points. Hence, although it is possible to obtain the parameters in the system with sufficient accuracy, it is not practical to use parameter identification to obtain system parameters for every circumstance.

The problem of designing adaptive control laws for rigid-robot manipulators with uncertainty that ensures asymptotic trajectory tracking has interested researchers for many years and many adaptive control algorithms have been proposed $[20,15$, $17,49,15,22]$. The basic idea of adaptive control is to change the values of gains or parameters in the control law according to some online algorithms corresponding to the changes and uncertainties in the system. The adaptive controller can learn an appropriate set of controller parameters during the course of its operation. A key point of the adaptive control algorithm is to make the tracking error converges 
regardless of whether the trajectory is persistently exciting or not [49]. That is, we do not need parameter convergence for task convergence. Moreover, the overall stability and convergence of the combined on-line control process can also be systematically guaranteed. This control approach is especially useful for robotic manipulators, which usually perform repetitive tasks. The tracking performance of the adaptive controller can improve with time through the adaptation for each successive operation [26].

In this study, we develop an adaptive actuator failure compensation scheme for a cooperative manipulator robotic system. The characteristic of the cooperative manipulator system is that it uses multiple manipulators to achieve an objective, such as moving or balancing an object. Because the cooperative manipulator system has multiple manipulators, it often has redundancy in the system. The redundancy allows is to develop an adaptive actuator failure compensation scheme for any possible failure in the system. Over the past two decades, cooperative manipulator systems have attracted the attention of many researchers. However, most literature focuses on the studies of kinematics and dynamics of the system $([8,9,10,11]$, researches on control strategies are relatively few, especially regarding to actuator failure compensation problem. In the literature, there are two types of basic control strategies for cooperative manipulator system: kinematic control strategies and dynamic control strategies. In the kinematic control strategies, the nonlinear dynamics are not considered to avoid the complex computation, thus, the controller can be easily designed. However, these controllers can not always produce high performance, and there is no guarantee of stability at the high speed. On the other hand, the dynamic control strategies, which are considered in this study, use a full dynamic model of the system. So the nonlinear dynamics of the manipulators can be compensated and higher performance can be achieved.

This topic makes the following contributions for the control and robotic field of 
study:

- Develop adaptive actuator failure compensation scheme for a class of cooperative manipulator system with parameter uncertainties, in addition to actuator failure uncertainties.

- Derive a complete proof and perform performance analysis of the proposed actuator failure compensation scheme.

- Develop a compensation scheme for a square-wave actuator failure signal in a cooperative manipulator system.

- Study the simulation of the adaptive actuator failure compensation scheme based on a benchmark cooperative manipulator system with parameter uncertainties.

The organization of this chapter is as follows. In Section 2, the formulation of the control problem is discussed. This includes the basic concepts and needed assumptions, such as the system model, the structure of actuator failure signal, control objective and actuator redundancy. In Section 3, nominal controller is developed for the system when the knowledge of actuator failures is known. In Section 4, we parameterize the nominal controller and develop an adaptive laws for the actuator failure compensation scheme. The complete proof and performance analysis is also given in this section. Finally in Section 5, we study the simulation of a cooperative manipulator benchmark system with both constant and square-wave actuator failure to confirm the effectiveness of the adaptive actuator failure compensation scheme. 


\subsection{Problem Formulation}

The aim of this study is to solve the actuator failure problem to increase reliability of robotic systems. The paper develops an adaptive actuator failure compensation scheme for cooperative manipulator systems with unknown system parameters. The proposed algorithm ensures the system stability and asymptotic tracking properties of robotic systems with possible actuator failure.

\subsubsection{Dynamic Model of Cooperative Manipulator Systems}

In this research, the cooperative manipulator robotic system is referred to many manipulators that is performing a single task together. For this type of system, we assume that the problem of system constraint is negligible because the base position of each manipulation or the gripping position of each manipulator can be moved, which enable some flexibilities in the system structure.

The general dynamic model of cooperative manipulator systems can be divided into two parts, which are the dynamic of each manipulator and the dynamic of the mutual platform. In order to develop a controller for a cooperative manipulator system, we need to obtain the combined dynamic of the system as discussed in $([?])$. In this study, we will develop an adaptive actuator failure compensation scheme for a general cooperative manipulator system with $n$ degrees of freedom and $m$ actuators. The combined dynamic model of the cooperative systems balancing a rigid platform can be described as follows.

$$
D_{c}\left(x_{o}\right) \ddot{x}_{o}+C_{c}\left(x_{o}, \dot{x}_{o}\right) \dot{x}_{o}+G_{c}\left(x_{o}\right)=E\left(x_{o}\right) \tau,
$$

where $x_{o} \in R^{n}$ is the output of the system representing the position and orientation 
of the mutual object, $D_{c}\left(x_{o}\right) \in R^{n \times n}$ is the combined inertia matrix of the object and manipulators, which is assumed to be a bounded and positive definite matrix, $C_{c}\left(x_{o}, \dot{x}_{o}\right) \in R^{n \times n}$ is the Coriolis and centrifugal term, $G_{c}\left(x_{o}\right) \in R^{n}$ is the gravity term, $E=\left[E_{1}, E_{2}, \ldots, E_{m}\right] \in R^{n \times m}$ is a transformation matrix from the joint space to the task space, $E_{i} \in R^{n}$ and $\tau=\left[\tau_{1}, \ldots, \tau_{m}\right]^{T}$ with $\tau_{i}$ as the torque of each actuator. Since the cooperative manipulator system need to carry various objects, some parameters in $D_{c}, C_{c}$ and $G_{c}$ are often unknown.

Although the parameters of the system are unknown, it is common to assume that the system satisfies the following structural properties:

a) $D_{c}$ is a symmetric and positive definite matrix.

b) $\dot{D}_{c}-2 C_{c}$ is a skew symmetric matrix.

\subsubsection{Actuator Failure Model}

In order to develop an adaptive actuator failure compensation scheme, we need to obtain the structure of the actuator failure signal. Although we do not have the knowledge of actuator failure, such as the failure value or failing time, we can model the failure signal structure as follows.

When actuator failure occurs, the control input components $\tau_{i}(t)$ corresponding with the failing actuator $i$ may become uncertain, the input components become

$$
\tau_{i}(t)=\bar{\tau}_{i}(t), t \geq t_{i}, i \in\{1,2, \ldots, m\},
$$

where $m$ is the number of actuators in the system and $t_{i}$ is the time that each actuator failure occurs. 
We model the actuator failure signal as

$$
\bar{\tau}_{i}(t)=\bar{\tau}_{i 0}+\sum_{j=1}^{\bar{n}} \bar{\tau}_{i j} f_{a i j}(t), t \geq t_{i}
$$

where $\bar{n}$ is the number of possible failing components in an actuator, $f_{a i j}$ are known functions corresponding to each failing component of the actuator and $i, t_{j}, \bar{\tau}_{i j}$ are unknown failure index, failure time and failure value of each failing actuator component.

With some specifications of the unknown constants $\bar{\tau}_{j 0}, \bar{\tau}_{i j}$ and known functions $f_{\text {aij }}(t)$, we can model most common actuator failures in the robotic system such as the complete failure of an actuator: $\bar{\tau}_{j}=0$, or the lock in-place failure of the motor: $\bar{\tau}_{j}=\bar{\tau}_{j 0}$, which may happen when the controller fails to communicate with the actuator. The terms $\bar{\tau}_{i j} f_{a i j}(t)$ can be used to represent time varying actuator failures signal. In this study, we mainly focus on the constant actuator failure signal as well as as the square wave actuator failure signal.

\subsubsection{Basic Assumption}

The adaptive actuator failure compensation scheme developed in this study utilizes the redundancy in the system to compensate for possible failures. In order to achieve this, a system with $n$ degrees of freedom needs at least $n$ functioning actuators. This means that a cooperative manipulator system (6.1) with $m$ actuators allows up to $k$ actuator to fail at the same time, where $k \leq m-n$. In this paper, we consider a benchmark cooperative manipulator system with two degrees of freedom and three actuators. The configuration of the benchmark system allows one actuator to fail. The proposed control algorithm needs the following assumption to guarantee that the solution for the actuator failure compensation scheme to exist. 
Assumption 6.1. Define a matrix $\bar{E}_{(i)}$ as a submatrix of $E\left(x_{o}\right)$ in (6.1) without the associated column $E_{i_{1}}, E_{i_{2}}, \ldots, E_{i_{k}}$, where $i_{j}$ is the actuator failure index of each actuator failure case for $j=1,2, \ldots, k$ with $k$ as the number of the concurrent actuator failures. In this study, we assume that the rank of the matrix $\bar{E}_{(i)}$ must be larger or equal to the degree of freedom $n$ for every actuator failure pattern.

This assumption prevents the possibility that the system becomes underactuated in some system configurations, even when the number of the remaining actuators is larger than the degrees of freedom. This concept can be clearly seen in the cooperative manipulator benchmark system discussed in the simulation study.

\subsubsection{Control Objective}

Our control objective is to ensure the orientation of the platform, or to make $x_{o}(t)$ tracks a desired trajectory $x_{d}(t)$ asymptotically. Consider the cooperative manipulator robotic model (6.1), due to uncertain actuator failures, an applied feedback control signal $\tau_{c}(t)=\left[\tau_{c 1}, \tau_{c 2}, \ldots, \tau_{c m}\right]^{T}$ may not reach the system, as $\tau_{i}(t)$ is not equal to $\tau_{c i}(t)$. More precisely, the signal $\tau(t)$ is

$$
\tau(t)=(I-\sigma(t)) \tau_{c}(t)+\sigma(t) \bar{\tau},
$$

where $\bar{\tau}$ is an unknown vector of the actuator failure values and

$\sigma(t)=\operatorname{diag}\left\{\sigma_{1}, \sigma_{2}, \ldots, \sigma_{m}\right\}$ is the matrix of actuator failure pattern such that $\sigma_{i}(t)=1$ if the $i$ actuator fails and $\sigma_{i}(t)=0$ otherwise.

The control objective is to design a feedback control signal $\tau_{c}(t)$ such that the closed-loop system $D_{c} \dot{s}+C_{c} s+K_{d} s=0$, despite the uncertain actuator failures 
$\tau(t)=(I-\sigma(t)) \tau_{c}(t)+\sigma(t) \bar{\tau}$, where the error vector $s$ is defined as $s=\dot{e}+\Lambda_{0} e$ with $e=x_{o}-x_{d}, x_{d}$ is the desired trajectory, $\Lambda_{0} \in R^{n \times n}$ is a design matrix whose eigenvalues have positive real parts and the gain matrix $K_{d}$ is chosen to be positive definite. This control objective ensures the desired signal boundedness and tracking properties of the system.

The algorithm proposed in this study can be used to compensate up to $k$ concurrent actuator failures. In this paper, we will first design allows up to one actuator to fail for simplicity. The design for multiple actuator failure will be discussed in Section 6.4.5. The adaptive actuator failure compensation scheme is designed to compensate for actuator failures without the knowledge of the failing actuator $i$, time that actuator failure occurs $t_{i}$, or value of the output of the failing actuator $\bar{\tau}_{j}(t)$, so that the system will remain stable and the output $x_{o}(t)$ asymptotically tracks the reference signal $x_{d}(t)$ even when actuator failures occur.

\subsection{Nominal control design}

In this section, we develop a baseline nominal controller for the cooperative manipulator robotic system. The nominal controller assumes that all knowledge of the actuator failures such as the failing pattern, failing time and failing values are known. The nominal controller structure will be used to design an adaptive actuator failure compensation scheme in the following section.

The nominal controller uses the integration of several controller bank, each controller bank is developed based on an existing control algorithm that is designed for the system without actuator failure such as the backstepping control or the Slotine and Li's control design. In this case, the actuator failure values are known, and the switching scheme is developed from the known actuator failure pattern. 


\subsubsection{Based Line Control Design}

Consider the dynamic model of the cooperative manipulator system (6.1)

$$
D_{c}\left(x_{o}\right) \ddot{x}_{o}+C_{c}\left(x_{o}, \dot{x}_{o}\right) \dot{x}_{o}+G_{c}\left(x_{o}\right)=E\left(x_{o}\right) \tau .
$$

If we can completely generate $E\left(x_{o}\right) \tau$, it is possible to design the baseline controller as follows.

Design for the known parameters case. If all parameters in the system are known, we can design a controller using the Slotine and Li's approach [18]. This controller is designed for the system without actuator failure. It will be used as a baseline for developing the adaptive actuator failure compensation scheme.

In this case, we first define an error vector $s=\dot{e}+\Lambda_{0} e$, where $e=x_{o}-x_{d}, x_{d}$ is the desired trajectory, $v=\dot{x}_{d}-\Lambda_{0} e$ being an intermediate vector signal and let $\Lambda_{0}$ be any $n \times n$ constant matrix whose eigenvalues have positive real parts. The definition of this error vector means that the convergence of $s$ also implies the convergence of the tracking error.

The cooperative manipulator model can be rewritten with the error vector as

$$
D_{c} \dot{s}+C_{c} s=E \tau-D_{c} \dot{v}-C_{c} v-G_{c} .
$$

Since we assume that all parameters are known and the rank of $E\left(x_{o}\right)$ is larger than $n$, we can alway choose a controller such that

$$
E \tau(t)=D_{c} \dot{v}+C_{c} v+G_{c}-K_{d} s(t),
$$


where the gain matrix $K_{d}$ is chosen to be a positive definite matrix. If we can precisely generate $E \tau(t)$, the closed-loop system becomes

$$
D_{c} \dot{s}+C_{c} s+K_{d} s=0
$$

From closed loop system (6.8) and the definition of $s$, we can guarantee that the system output $x_{o}$ tracks the desired trajectory $x_{d}$ asymptotically. In this case, the controller needs complete knowledge of parameters in the system.

Parameterization for Unknown Parameters. Since robotic systems often interact with environment, some parameters in $D_{c}, C_{c}$ and $G_{c}$ in often unknown. To design a controller for the system with parameter uncertainties, we estimate the unknown matrices with $\hat{D}, \hat{C}, \hat{G}$ respectively. With this equation (6.7) can be written as

$$
E \tau(t)=\hat{D} \dot{v}+\hat{C} v+\hat{G}-K_{d} s(t)
$$

We can linearly parameterize the system by rewriting the right side of equation (6.9) as a known regressor matrix $Y\left(x_{o}, \dot{x}_{o}, v, \dot{v}\right)$ multiplied by an estimate $\hat{\theta}$ of an unknown parameter vector, the controller becomes

$$
E \tau=\hat{D} \dot{v}+\hat{C} v+\hat{G}-K_{d} s=Y \hat{\theta}-K_{d} s
$$

where the regressor matrix $Y\left(x_{o}, \dot{x}_{o}, v, \dot{v}\right)$ is such that

$$
D_{c} \dot{v}+C_{c} v+G_{c}=Y\left(x_{o}, \dot{x}_{o}, v, \dot{v}\right) \theta^{*}
$$

with $\theta^{*}$ being the unknown parameter vector in the system that is estimated by $\hat{\theta}$. 
The closed-loop system becomes

$$
D_{c} \dot{s}+C_{c} s+K_{d} s=Y \tilde{\theta},
$$

where $\tilde{\theta}=\hat{\theta}-\theta^{*}$ is the estimation error of the unknown parameters in the system.

With the following parameterization, we can design a nominal controller for the system where the information of actuator failure is known.

\subsubsection{Actuator Failure Compensation}

In this study, we consider the cooperative manipulator system (6.1), whose actuators are subjected to unknown actuator failures as model in (6.3). That is, to design a feedback control signal $\tau_{c}(t)$ to make the system output $x_{o}(t)$ tracks a desirable trajectory $x_{d}(t)$ asymptotically.

In order to achieve this, we first consider the nominal design (6.7) with the regressor matrix (6.11) as

$$
E\left(x_{o}\right) \tau=Y\left(x_{o}, \dot{x}_{o}, v, \dot{v}\right) \theta^{*}-K_{d} s(t) .
$$

When an actuator failure occurs the feedback control signal $\tau_{c}(t)$ may not reach the system as $\tau=(I-\sigma) \tau_{c}+\sigma \bar{\tau}$, so equation (6.13) becomes

$$
E\left[(I-\sigma(t)) \tau_{c}(t)+\sigma(t) \bar{\tau}\right]=Y \theta^{*}-K_{d} s(t) .
$$

In order to control the system, our task is to design the feedback control signal $\tau_{c}(t)$ such that $E \tau(t)=Y(q, \dot{q}, v, \dot{v}) \theta^{*}-K_{d} s(t)$ so that $D_{c} \dot{s}+C_{c} s+K_{d} s=0$. for every possible actuator failure pattern.

To demonstrate this design technique, we will consider a general cooperative ma- 
nipulator system with $m$ actuators and $n$ degrees of freedom with $m=n+1$. This allows one actuator failure to fail at any time. This design will be further illustrated with a benchmark cooperative manipulator system in the simulation study section.

Our proposed actuator failure compensation scheme chooses a controller structure as a combination of multiple nominal controller of each actuator failure pattern. According the Slotine and Li's control algorithm, the individual controller structure for each actuator failure case can be designed as follows.

Design for the no failure case. In the no failure case, we have the control signal $\tau(t)=\tau_{c}^{*}(t)$. We design the feedback control signal $\tau_{c}^{*}(t)$ such that $E\left(x_{o}\right) \tau_{c}^{*}(t)=$ $Y\left(x_{o}, \dot{x}_{o}, v, \dot{v}\right) \theta^{*}-K_{d} s(t)$. We consider

$$
\tau_{c}^{*}(t)=\tau_{c(0)}^{*}(t)=h_{a}\left(x_{o}\right) \tau_{c a(0)}^{*}(t)
$$

for some chosen matrix function $h_{a}\left(x_{o}\right) \in R^{m \times m}$. We need to choose the matrix $h_{a}$ such that the function $\bar{E}_{(0)} h_{a}$ is invertible. For example, if the matrix $\bar{E}_{(0)}$ is a not square matrix, then we will need to choose $h_{a}$ such that $\bar{E} h_{a}$ is a square matrix and is invertible. Furthermore, the design matrix $h_{a}$ can also be used for the load distribution of actuators in the system. The signal $\tau_{c a(0)}^{*}(t) \in R^{m \times 1}$ to be determined from

$$
E\left(x_{o}\right) h_{a}\left(x_{o}\right) \tau_{c a(0)}^{*}(t)=Y\left(x_{o}, \dot{x}_{o}, v, \dot{v}\right) \theta^{*}-K_{d} s(t) .
$$

In this case, we can choose $h_{a}\left(x_{o}\right)$, such that the solution for $\tau_{c a(0)}^{*}(t)$ exists. The explicit form of the solution $\tau_{c a(0)}^{*}(t)$ can be written as

$$
\tau_{c a(0)}^{*}(t)=K_{a 1}\left(x_{o}\right)\left(Y \theta^{*}-K_{d} s(t)\right)
$$

for some $K_{a 1} \in R^{m \times n}$. Since the matrix $K_{a 1}$ has more rows than its columns, this 
structure of $K_{a i}$ admits possibility up to $m-n$ constrained optimality. For unique solution, we choose the controller by using only two actuators to control the system. To achieve this, we set $\tau_{c 1}=0$, the controller is

$$
\tau_{c(0)}^{*}(t)=\left[\begin{array}{ll}
0 & \tau_{c b(0)}^{* T}(t)
\end{array}\right]^{T}, \tau_{c b(0)}^{*}(t)=h_{0}\left(x_{o}\right) \tau_{c 0(0)}^{*}(t) .
$$

Define a submatrix $\bar{E}_{(0)}=\left[E_{2}, E_{3}, \ldots, E_{m}\right]$, where $E_{i}$ is the $i$ column of the transformation matrix $E\left(x_{o}\right)$. In this case, a new matrix function $h_{0}\left(x_{o}\right) \in R^{n \times n}$ can be chosen such that $\bar{E}_{(0)} h_{0}\left(x_{o}\right)$ is invertible. With Assumption 1, the explicit form of $\tau_{c 0(0)}^{*}(t) \in R^{n \times 1}$ can be written as

$$
\tau_{c 0(0)}^{*}(t)=K_{01}\left(x_{o}\right)\left(Y \theta^{*}-K_{d} s(t)\right)
$$

where the matrix $K_{01}\left(x_{o}\right) \in R^{n \times n}$ are expressed as

$$
K_{01}\left(x_{o}\right)=\left(\bar{E}_{(0)}\left(x_{o}\right) h_{0}\left(x_{o}\right)\right)^{-1}
$$

This design ensures $E\left(x_{o}\right) \tau=Y\left(x_{o}, \dot{x}_{o}, v, \dot{v}\right) \theta^{*}-K_{d} s(t)$ for the no failure case.

Design for one actuator failure case. In case of the actuator $i$ fails, we have $\tau_{i}=\bar{\tau}_{i}, \tau_{j}=\tau_{c j}^{*}$ for $j=1,2, \ldots, m$ with $j \neq i$. The nominal control signal can be chosen as

$$
\tau_{c}^{*}(t)=\tau_{c(i)}^{*}(t)=\left[\alpha_{1} \tau_{c a(i) 1}^{*}, \alpha_{2} \tau_{c a(i) 2}^{*}, \ldots, \alpha_{n} \tau_{c a(i) m}^{*}\right]^{T}
$$

where $\alpha_{i}=0$ if the actuator $i$ fails and $\alpha_{i}=1$ otherwise. Define the intermediate control signal $\tau_{c a(i)}^{*}$ as a vector consisting of the non-zero rows of $\tau_{c(i)}^{*}$. The intermediate control signal $\tau_{c a(i)}^{*}$ can be chosen as

$$
\tau_{c a(i)}^{*}(t)=h_{i}\left(x_{o}\right) \tau_{c 0(i)}^{*}(t),
$$


where the matrix $h_{i}\left(x_{o}\right) \in R^{n \times n}$ is chosen such that $\bar{E}_{(i)} h_{i}\left(x_{o}\right)$ is invertible and the signal $\tau_{c 0(i)}^{*}(t) \in R^{n \times 1}$ can be obtained from

$$
E_{i} \bar{\tau}+\bar{E}_{(i)} h_{i}\left(x_{o}\right) \tau_{c 0(i)}^{*}(t)=Y \theta^{*}-K_{d} s(t)
$$

The explicit form of the signal $\tau_{c 0(i)}^{*}(t)$ can be written as

$$
\tau_{c 0(i)}^{*}(t)=K_{i 1}\left(x_{o}\right)\left(Y \theta^{*}-K_{d} s(t)\right)+K_{i 2}\left(x_{o}\right) \bar{\tau}_{i}
$$

where $K_{i 1} \in R^{n \times n}, K_{i 2} \in R^{n \times 1}$ are expressed as

$$
K_{i 1}=\left(\bar{E}_{(i)} h_{i}\left(x_{o}\right)\right)^{-1}, K_{i 2}=-\left(\bar{E}_{(i)} h_{i}\left(x_{o}\right)\right)^{-1} E_{i}\left(x_{o}\right)
$$

It is easy to see that the nominal controller $\tau_{c(i)}^{*}(t)$ ensures that $E\left(x_{o}\right) \tau=Y\left(x_{o}, \dot{x}_{o}, v, \dot{v}\right) \theta^{*}-$ $K_{d} s(t)$ for each actuator failure case.

Composite control design. With the control structure for the no actuator failure case and $m$ cases of one actuator failure case, we can design a composite control law for the system by combining all cases as proposed in [?] and [?] as

$$
\tau_{c}^{*}(t)=\chi_{0}^{*} \tau_{c(0)}^{*}(t)+\sum_{i=1}^{m} \chi_{i}^{*} \tau_{c(i)}^{*}(t)
$$

where $\chi_{0}^{*}, \chi_{i}^{*}$ for $i=1, \ldots, m$ are the indicator functions of the actuator failure: $\chi_{0}^{*}=1$ when there is no actuator failure, $\chi_{i}^{*}=1$ for $i$ actuator failure. The indicator functions $\chi_{0}^{*}, \chi_{i}^{*}=0$ for its non-corresponding cases.

With the knowledge of the indicator functions and the nominal control signals, this design ensures $E\left(x_{o}\right) \tau=Y\left(x_{o}, \dot{x}_{o}, v, \dot{v}\right) \theta^{*}-K_{d} s(t)$ for every case of actuator failure, which implies that the tracking error converges to zero asymptotically. However, 
it is very difficult to obtain the knowledge of which actuator has failed in real time. In the next section, we will develop an adaptive actuator failure compensation scheme for the system without the knowledge of which actuator has failed.

Remark 6.1: As previously mention, our adaptive actuator failure compensation scheme proposed in this paper is applicable with various baseline control approaches. The proposed scheme extends the capability of state of the art control technique to handle actuator failure problems of robotic systems. In this subsection, an alternative baseline control algorithm using backstepping control technique is considered as a comparison to the Slotine and Li's control algorithm.

With the backstepping design, we need to design the adaptive actuator failure compensation to ensure $D_{c}^{-1} E\left(x_{1}\right) \tau=-D_{c}^{-1}\left(-C_{c}-G_{c}\right) x_{2}-c_{2} z_{2}-z_{1}+\dot{\beta}$. If all parameters of the system are known, we can directly design actuator failure compensation scheme with this algorithm. The cancellation of the nonlinear terms in the backstepping control design could improve the system transient response of the adaptive actuator failure compensation scheme. The disadvantage of this approach is that the controller structure needs the inverse of the inertia matrix $D_{c}^{-1}$, which may be difficult to obtain, especially for a robotic system with parameter uncertainties.

\subsection{Adaptive Failure Compensation Design}

The nominal control design derived in the previous section needs the knowledge of the actuator failures as well as the values of every parameter in the system, which can be difficult to obtain. In this section, the adaptive control scheme is developed based on the nominal controller structure and parameterization for failure compensation.

In the adaptive failure compensation design, an adaptive integration of multiple 
individual failure compensators is used to combine the controllers. Since the actuator failure pattern is unknown, the switching algorithm is integrated into each adaptive controller. The control input $\tau_{c}(t)$ is the combination of each control scheme based on the nominal controller from the previous section, which each controller is developed using the Slotine and Li's control algorithm. There is an estimator corresponding to each controller, the estimators estimate the actuator failure values, actuator failure patterns and the uncertain parameters in the system. In this section, we will explain the parameterization of the controller and develop adaptive laws for the estimators.

\subsubsection{Adaptive Controller Structure}

The adaptive control scheme is developed based on the nominal controller structure (6.26). Since we do not know the values of $\chi_{0}^{*}, \chi_{i}^{*}, \bar{\tau}$ and $\theta^{*}$, the adaptive controller is constructed as

$$
\tau_{c}(t)=\tau_{c \chi(0)}(t)+\sum_{i=1}^{m} \tau_{c \chi(i)}(t),
$$

where $\tau_{c \chi(0)}(t)$ and $\tau_{c \chi(i)}(t)$ are the estimates of $\chi_{0}^{*} \tau_{c(0)}^{*}$ and $\chi_{i}^{*} \tau_{c(i)}^{*}$ respectively.

We need to parameterize each individual term in the adaptive controller structure. To derive $\tau_{c \chi(0)}(t)$, we restructure $\chi_{0}^{*} \tau_{c b(0)}^{*} \in R^{n \times 1}$ from the corresponding nominal design (6.18) and (6.19) as

$$
\chi_{0}^{*} \tau_{c b(0)}^{*}=-\chi_{0}^{*} h_{0} K_{01} K_{d} s(t)+h_{0} K_{01} Y \chi_{0}^{*} \theta^{*}
$$

Since the term $\chi_{0}^{*}$ is a scalar multiplied with a matrix. We need to parameterize this term multiple times to reflect the multiplication to each row of the matrix. Redefining $\chi_{0}^{*}$ as $\chi_{0}^{*}=\operatorname{diag}\left\{\chi_{01}^{*}, \chi_{02}^{*}, \ldots, \chi_{0 n}^{*}\right\}$ with $\chi_{01}^{*}=\chi_{02}^{*}=\ldots=\chi_{0 n}^{*}=\chi_{0}^{*}$, we can choose 
the adaptive controller structure as $\tau_{c \chi(0)}(t)=\left[0, \tau_{c \chi b(0)}^{T}(t)\right]^{T}$, where

$$
\tau_{c \chi b(0)}(t)=-\operatorname{diag}\left\{\chi_{01}, \ldots, \chi_{0 n}\right\} h_{0} K_{01} K_{d} s(t)+h_{0} K_{01} Y \theta_{0}
$$

with $\theta_{0}$ is the estimate of $\chi_{0}^{*} \theta^{*}$ and $\chi_{0 j}$ is the estimate of $\chi_{0 j}^{*}$ for $j=1,2, \ldots, n$.

To derive $\tau_{c \chi(i)}(t)$, we first define $\tau_{c \chi(i)}(t)=\left[\alpha_{1} \tau_{c \chi(i) 1}, \alpha_{2} \tau_{c \chi(i) 2}, \ldots, \alpha_{3} \tau_{c \chi(i) n}\right]^{T}$, where $\alpha_{i}=0$ if actuator $i$ fails. Then, we calculate the elements of $\tau_{c \chi(i)}(t)$ from its subvector $\tau_{c \chi a(i)}$. The vector $\tau_{c \chi a(i)}$ is the same as $\tau_{c \chi(i)}(t)$ without the $i$ column. The vector $\tau_{c \chi a(i)}$ is developed by considering its nominal structure as

$$
\tau_{c a(i)}^{*}(t)=-\chi_{(i)}^{*} h_{i} K_{i 1} K_{d} s+h_{i} K_{i 1} Y \chi_{(i)}^{*} \theta^{*}+\chi_{(i)}^{*} \bar{\tau}_{i} h_{i} K_{i 2} .
$$

Because of the unknown actuator failure signal $\bar{\tau}_{i}$, we need to use the actuator failure model (6.3). The actuator failure signal can be expressed as $\bar{\tau}_{i}(t)=\rho_{i}^{* T} f_{a i}(t)$, where $f_{a i}(t)=\left[1, f_{a i 1}(t), \ldots, f_{a i \bar{n}}(t)\right]^{T}$ are known functions corresponding to the failure components, $\rho_{i}^{*}=\left[\bar{\tau}_{i 0}, \bar{\tau}_{i 1}, \ldots, \bar{\tau}_{i \bar{n}}\right]^{T}$ contains the parameter values associated with each failing component of the actuator and $\bar{n}$ is the number of possible failing components of an actuator.

With similar parameterization of $\chi_{i}^{*}$ and the actuator failure model, we can choose the controller $\tau_{c \chi a(i)}(t)$ as

$$
\tau_{c \chi a(i)}(t)=-\operatorname{diag}\left\{\chi_{i 1}, \ldots, \chi_{i n}\right\} h_{i} K_{i 1} K_{d} s(t)+h_{i} K_{i 1} Y \theta_{i}+\left[\begin{array}{c}
\rho_{i(1)}^{T} f_{a i}(t) \phi_{i 1} \\
\rho_{i(2)}^{T} f_{a i}(t) \phi_{i 2}, \\
\vdots \\
\rho_{i(n)}^{T} f_{a i}(t) \phi_{i n}
\end{array}\right] \text {, }
$$


where $\theta_{i}$ is the estimate of $\chi_{i}^{*} \theta^{*}$ and $\chi_{i j}$ is the estimate of $\chi_{i j}^{*}$ for $j=1,2, \ldots, n$. The terms $\chi_{i j}^{*} \rho_{i}^{*}$ is estimated by $\rho_{i(j)}$ for $j=1,2, \ldots, n$, where $\phi_{i}=\left[\phi_{i 1}, \phi_{i 2}, \ldots, \phi_{i n}\right]^{T}=$ $h_{i} K_{i 2}$.

\subsubsection{Error System}

In order to design an adaptive law, we consider the estimation error from the estimates of actuator failures and unknown parameters. Our adaptive actuator failure compensation design ensures

$$
\begin{aligned}
E \tau(t) & =E(I-\sigma) \tau_{c}+E \sigma \bar{\tau} \\
& =E(I-\sigma)\left(\tau_{c}-\tau_{c}^{*}\right)+E(I-\sigma) \tau_{c}^{*}+E \sigma \bar{\tau} \\
& =E(I-\sigma)\left(\tau_{c}-\tau_{c}^{*}\right)+Y \theta^{*}-K_{d} s(t)
\end{aligned}
$$

as $E(I-\sigma) \tau_{c}^{*}+E \sigma \bar{\tau}=Y \theta^{*}-K_{d} s(t)$ is ensured by the nominal design. We have

$$
E \tau(t)=Y \theta^{*}-K_{d} s(t)+E(I-\sigma(t))\left(\tau_{c}-\tau_{c}^{*}\right)
$$

From equation (6.32) and (6.33), the closed-loop system becomes

$$
D_{c} \dot{s}+C_{c} s+K_{d} s=E(I-\sigma(t))\left(\tau_{c}-\tau_{c}^{*}\right) .
$$

The error signal $\tilde{\tau}_{c}=\tau_{c}-\tau_{c}^{*}$ can be written as

$$
\tilde{\tau}_{c}=\left[0,\left(-\tilde{\chi}_{0} h_{0} K_{01} K_{d} s(t)+h_{0} K_{01} Y \tilde{\theta}_{0}\right)^{T}\right]^{T}+\sum_{i=1}^{m} \tilde{\tau}_{c(i)},
$$


where $\tilde{\tau}_{c(i)}=\left[\alpha_{1} \tilde{\tau}_{c a(i) 1}(t), \alpha_{2} \tilde{\tau}_{c a(i) 2}(t), \alpha_{3} \tilde{\tau}_{c a(i) 3}(t)\right]^{T}, \tilde{\chi}_{i}=\operatorname{diag}\left\{\tilde{\chi}_{i 1}, \ldots, \tilde{\chi}_{i n}\right\}, \tilde{\chi}_{i j}=\chi_{i j}-$ $\chi_{i}^{*}$ and the error signal $\tilde{\tau}_{c a(i)}$, which represents the nonzero term of $\tilde{\tau}_{c a(i)}$ is

$$
\tilde{\tau}_{c a(i)}=-\tilde{\chi}_{i} h_{i} K_{i 1} K_{d} s(t)+h_{i} K_{i 1} Y \tilde{\theta}_{i}+\left[\begin{array}{c}
\tilde{\rho}_{i(1)}^{T} f_{a i}(t) \phi_{i 1} \\
\tilde{\rho}_{i(2)}^{T} f_{a i}(t) \phi_{i 2} \\
\vdots \\
\tilde{\rho}_{i(n)}^{T} f_{a i}(t) \phi_{i n}
\end{array}\right]
$$

with the error signals $\tilde{\rho}_{i(j)}=\rho_{i(j)}-\chi_{i}^{*} \rho_{i}^{*}$ and $\tilde{\theta}_{i}=\theta-\chi_{i}^{*} \theta^{*}$ for $j=1,2, \ldots, n$.

\subsubsection{Adaptive Laws}

In this subsection, we develop an adaptive law for updating the estimated parameters (6.27). The adaptive law is developed based on the error system, which can guarantee the desired system performance. The adaptive laws for $\chi_{i j}$ with $i=0,1, \ldots, m$ and $j=1,2, \ldots, n$ are chosen as

$$
\dot{\chi}_{i j}=-\gamma_{i j} s^{T} \bar{E}_{(i) j} \mu_{i j}+\varrho_{\chi_{i j}}
$$

where $\bar{E}_{(i) j} \in R^{n \times 1}$ represents the $j$ column of matrix $\bar{E}_{(i)}, \gamma_{i j}>0$ are the adaptation gains and

$$
\mu_{i}=\left[\mu_{i 1}, \mu_{i 2}, \ldots, \mu_{i n}\right]^{T}=-h_{i} K_{i 1} K_{d} s(t)
$$

with $\varrho_{\chi i j}$ as the projection function, $h_{i}$ and $K_{i 1}$ being the design matrices from the controller structure (??).

Similarly, the adaptive laws for $\rho_{i(j)}=\left[\rho_{i(j)(1)}, \ldots, \rho_{i(j)(\bar{n})}\right]^{T}$ become

$$
\dot{\rho}_{i(j)}=-\Gamma_{(i j)} f_{a j} s^{T} \bar{E}_{(i) j} \phi_{i j}+\varrho_{\rho_{i(j)}},
$$


where $\varrho_{\rho i(j)}$ is the projection function and $\Gamma_{(i j)}=\Gamma_{(i j)}^{T}>0$ are the adaptation gain matrices.

The adaptive laws for $\theta_{i j}$, where $j=1,2, \ldots, \bar{m}$ with $\bar{m}$ being the number of the unknown parameters, are chosen as

$$
\dot{\theta}_{i j}=-\beta_{i j} s^{T} \bar{E}_{(i)} h_{i} K_{i 1} Y_{j}+\varrho_{\theta_{i}},
$$

where $\varrho_{\theta_{i}}$ is the projection function, $Y_{j} \in R^{n \times 1}$ represents the $j$ column of the regressor matrix $Y\left(x_{o}, \dot{x}_{o}, v, \dot{v}\right)$ and $\beta_{(i j)}>0$ are the adaptation gains.

Remark 6.2: Here we use a parameter projection design for $\varrho_{\chi}(t), \varrho_{\theta}(t)$ and $\varrho_{\rho}(t)$.

We first consider the physical range of the indicator functions $\chi_{i}^{*}$, for $i=0,1, \ldots, n$. From the definition of the indicator functions, we have

$$
0 \leq \chi_{i}^{*} \leq 1
$$

Because $\chi_{i}^{*}=\operatorname{diag}\left\{\chi_{i 1}^{*}, \chi_{i 2}^{*}, \ldots, \chi_{i n}^{*}\right\}$ with $\chi_{i 1}^{*}=\chi_{i 2}^{*}=\ldots=\chi_{i n}^{*}=\chi_{i}^{*}$, we have $0 \leq \chi_{i j}^{*} \leq 1$. We can see that the estimated parameters $\chi_{i j}$ should also have the lower bound at 0 and the upper bound at 1 . The projection function $\varrho_{x i j}$ is chosen as

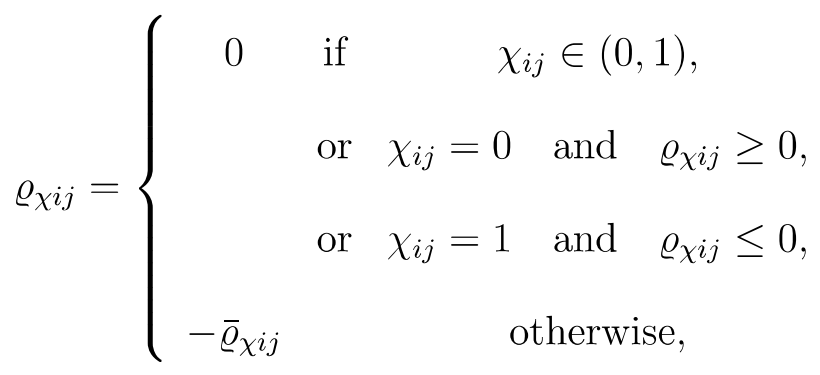

with $\bar{\varrho}_{\chi i j}$ defined as $\bar{\varrho}_{\chi i j}=\dot{\chi}_{i j}=-\gamma_{i j} s^{T} \bar{E}_{(i) j} \mu_{i j}$.

Let $\rho_{i(j)}^{* a}, \rho_{i(j)}^{* b}$ be the upper and lower bounds of the actuator failure components 
$\rho_{i(j)}^{*}$ that is,

$$
\rho_{i(j)}^{* a} \leq \rho_{i(j)}^{*} \leq \rho_{i(j)}^{* b} .
$$

The upper and lower bounds $\rho_{i(j)}^{* a}, \rho_{i(j)}^{* b}$ can be obtained from the maximum and minimum values that each failing actuator can produce based on the actuator failure model.

With the upper and lower bounds of each component of $\rho_{i}$, we can see that each component of the estimated parameters $\rho_{i(j)}$ should also have the same boundaries as the component of $\rho_{i}$ because $\rho_{i(j)}$ is the estimate of $\rho_{i} \chi_{j}$ with $0 \leq \chi_{j} \leq 1$.

The projection function $\varrho_{\rho i(j)}=\left[\varrho_{\rho i(j)(1)}, \ldots, \varrho_{\rho i(j)(\bar{n})}\right]^{T}$ is chosen as

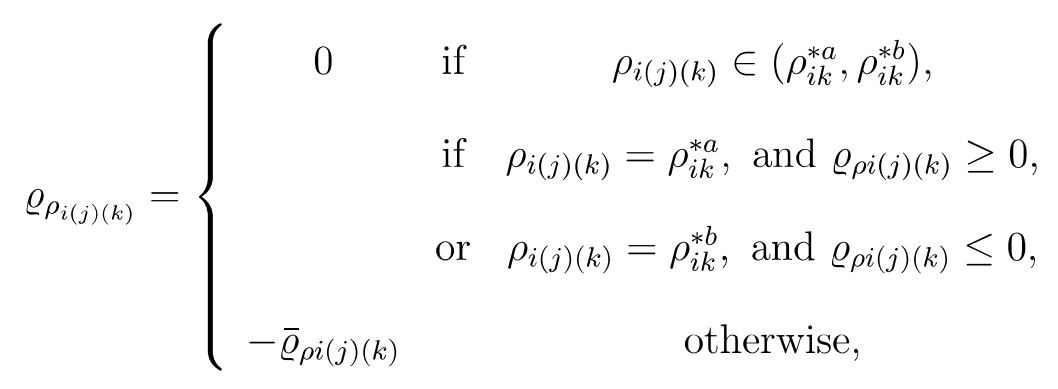

with $k=1,2, \ldots, \bar{n}$ and $\bar{\varrho}_{\rho i(j)}=\left[\bar{\varrho}_{\rho i(j)(1)}, \ldots, \bar{\varrho}_{\rho i(j)(\bar{n})}\right]^{T}$ is defined as $\bar{\varrho}_{\rho i(j)}=-\Gamma_{(i j)} f_{a j} s^{T} \bar{E}_{(i) j} \phi_{i j}$. The parameter projection scheme for $\varrho_{\theta}(t)$ can be designed in a similar way using $\theta^{* a}, \theta^{* b}$ as the upper and lower bounds of each component in $\theta^{*}$.

This choice of $\varrho_{\chi}(t), \varrho_{\theta}(t)$ and $\varrho_{\rho}(t)$ ensures that $\left(\chi_{i j}-\chi_{i j}^{*}\right) \varrho_{\chi_{i j}} \leq 0,\left(\theta_{i}-\right.$ $\left.\chi_{i}^{*} \theta^{*}\right) \varrho_{\theta}(t) \leq 0$ and $\left(\rho_{i(j k)}-\chi_{i j}^{*} \rho_{i k}^{*}\right) \varrho_{\rho_{i(j k)}} \leq 0$, which guarantee the boundedness of estimated parameters $\chi_{i j} \in L^{\infty}, \theta_{i j} \in L^{\infty}$ and $\rho_{i(j)} \in L^{\infty}$. 


\subsubsection{Performance Analysis}

The proposed actuator failure compensation scheme can ensure the stability and tracking of the system with uncertain actuator failures as shown by the following theorem.

Theorem 6.1. The adaptive actuator failure compensation scheme with the feedback control law (6.27) updated by the adaptive laws (6.37) - (6.40), when applied to the cooperative manipulator model (6.1) with up to one actuator failure, guarantees the closed-loop signal boundedness and asymptotic output tracking: $\lim _{t \rightarrow \infty} e(t)=0$, despites the uncertainties of actuator failure index, failure times and failure values.

Proof: We first consider the closed-loop system with the nominal controller $E\left(x_{o}\right) \tau_{c}^{*}(t)=$ $Y\left(x_{o}, \dot{x}_{o}, v, \dot{v}\right) \theta^{*}-K_{d} s(t)$. We can easily obtain the closed-loop system as

$$
D_{c} \dot{s}+C_{c} s+K_{d} s=0 .
$$

By comparing the adaptive failure compensation design with the nominal controller design as shown in the Error System section $E \tau(t)=Y \theta^{*}-K_{d} s(t)+E(I-\sigma(t))\left(\tau_{c}-\right.$ $\left.\tau_{c}^{*}\right)$, we have the closed-loop system as

$$
D_{c} \dot{s}+C_{c} s+K_{d} s=E(I-\sigma(t))\left(\tau-\tau_{c}^{*}\right) .
$$

The error signal $\tilde{\tau}_{c}=\tau_{c}-\tau_{c}^{*}$ is

$$
\tilde{\tau}_{c}=\left[0,\left(-\tilde{\chi}_{0} h_{0} K_{01} K_{d} s(t)+h_{0} K_{01} Y \tilde{\theta}_{0}\right)^{T}\right]^{T}+\sum_{i=1}^{m} \tilde{\tau}_{c(i)},
$$


where $\tilde{\chi}=\operatorname{diag}\left\{\tilde{\chi}_{i 1}, \ldots, \tilde{\chi}_{i n}\right\}, \tilde{\chi}_{i j}=\chi_{i j}-\chi_{i}^{*}$ and

$$
\tilde{\tau}_{c a(i)}=-\tilde{\chi}_{i} h_{i} K_{i 1} K_{d} s(t)+h_{i} K_{i 1} Y \tilde{\theta}_{i}+\left[\begin{array}{c}
\tilde{\rho}_{i(1)}^{T} f_{a i}(t) \phi_{i 1} \\
\tilde{\rho}_{i(2)}^{T} f_{a i}(t) \phi_{i 2} \\
\vdots \\
\tilde{\rho}_{i(n)}^{T} f_{a i}(t) \phi_{i n}
\end{array}\right]
$$

with $\tilde{\rho}_{i(j)}=\rho_{i(j)}-\chi_{i}^{*} \rho_{i}^{*}$ and $\tilde{\theta}_{i}=\theta-\chi_{i}^{*} \theta^{*}$ for $j=1,2, \ldots, n$.

To find a Lyapunov-like function candidate, we first consider the time derivative of $\frac{1}{2} s^{T} D_{c} s$ along the trajectory of (6.46), we have

$$
\frac{\partial}{\partial t}\left(\frac{1}{2} s^{T} D_{c} s\right)=-s^{T} K_{d} s+s^{T} E(I-\sigma(t)) \tilde{\tau}_{c}
$$

Based on the proposed adaptive laws, one can see that there are suitable Lyapunov function candidates that can be used to prove the stability of the system of each failure pattern $\sigma(t)$. The Lyapunov function can be chosen based on the adaptive law to canceled the non-negative term $s^{T} E(I-\sigma(t)) \tilde{\tau}_{c}$ in (6.59) for each case of actuator failure.

For the no actuator failure case, the Lyapunov function can be chosen as

$$
\begin{aligned}
V_{0}=\frac{1}{2} s^{T} D_{c} s & +\frac{1}{2} \sum_{i=0}^{m} \sum_{j=1}^{n} \tilde{\chi}_{i j}^{2} \gamma_{i j}^{-1} \\
& +\frac{1}{2} \sum_{i=1}^{m} \sum_{j=1}^{\bar{m}} \tilde{\theta}_{i j}^{T} \beta_{i j}^{-1} \tilde{\theta}_{i j}+\frac{1}{2} \sum_{i=1}^{m} \sum_{j=1}^{n} \tilde{\rho}_{i(j)}^{T} \Gamma_{i j}^{-1} \tilde{\rho}_{i(j)} .
\end{aligned}
$$

Based on the adaptive laws and the error equation (6.47), the time derivative of $\tilde{\chi}_{i j}^{2} \gamma_{i j}^{-1}, \tilde{\theta}_{i}^{T} \beta_{i}^{-1} \tilde{\theta}_{i}$ and $\tilde{\rho}_{i(j)}^{T} \Gamma_{i j}^{-1} \tilde{\rho}_{i(j)}$ canceled the non-negative definite terms in (6.59). 
The time derivative of the Lyapunov function becomes

$$
\dot{V}_{0} \leq-s^{T} K_{d} s \leq 0
$$

Let $k$ be the actuator failure index. The Lyapunov function for one actuator failure case is

$$
\begin{gathered}
V_{i}=\frac{1}{2} s^{T} E^{T} D_{c} E s+\frac{1}{2} \sum_{i=0}^{m} \sum_{j=1}^{n} \tilde{\chi}_{i j}^{2} \gamma_{i j}^{-1}+\frac{1}{2} \sum_{i=0}^{m} \sum_{j=1}^{m} \tilde{\theta}_{i j}^{T} \beta_{i j}^{-1} \tilde{\theta}_{i j} \\
+\frac{1}{2} \sum_{i=1}^{m} \sum_{j=1}^{n} \tilde{\rho}_{i(j)}^{T} \Gamma_{i j}^{-1} \tilde{\rho}_{i(j)}-\frac{1}{2} \sum_{i=0}^{m} \tilde{\chi}_{i k}^{2} \gamma_{i k}^{-1} \\
\quad-\frac{1}{2} \sum_{i=0}^{m} \tilde{\theta}_{i k}^{T} \beta_{i k}^{-1} \tilde{\theta}_{i k}-\frac{1}{2} \sum_{i=1}^{m} \tilde{\rho}_{i(k)}^{T} \Gamma_{i k}^{-1} \tilde{\rho}_{i(k)}
\end{gathered}
$$

The time derivative of the Lyapunov function $V_{i}$ is

$$
\dot{V}_{i} \leq-s^{T} K_{d} s \leq 0
$$

Let $\left(T_{i}, T_{i+1}\right), i=0,1,2, \ldots, n$ with $T_{0}=0, T_{n}=\infty$ be time intervals on which the actuator failure pattern is fixed. In the analysis we have proven that for each actuator failure pattern, there exists a Lyapunov function that ensures the performance of the system during $\left(T_{i}, T_{i+1}\right)$, where $T_{i}$ is the time that the an actuator failure occurs. Because we have considered all possible actuator failure cases, thus, we can guarantee the performance of the system for all time $\left(T_{0}, T_{n}\right)$.

With the Lyapunov function and the properties of the parameter projection scheme, we can show that $s(t) \in L^{\infty} \bigcap L^{2}$ and all signals in the system are bounded, based on the Barbalat lemma we can conclude that the error vector $\lim _{t \rightarrow \infty} s(t)=0 . \quad \nabla \nabla \nabla$

From Theorem 6.1, we can see that the adaptive actuator failure compensation scheme can guarantee the asymptotic tracking for the system with parameter uncertainties subjected to uncertain actuator failures, that is, for the system operating with any actuator failure pattern that we have considered. 


\subsubsection{Concurrent Actuator Failures}

A cooperative robotic system often uses serial-link structures to communicate between the controller and multiple actuators in the system. If there is a damage in a part of the communication link, it may cause every actuator in the same communication chain to fail at the same time. The concurrent actuator failures, where more than one actuator fails at the same time, is a crucial problem in the robotic field. Previously, the proposed control algorithm only considers single actuator failure cases, it is possible to extend the proposed actuator failure compensation scheme to concurrent actuator failure cases. For example, if one more actuator is added to the system, so that $m-n=2$. With the additional manipulator, the system has redundancy that allows up to two actuators to fail concurrently.

In order to develop an adaptive actuator failure compensation scheme that allows up to two actuators to fail at the same time, we first design a failure compensation scheme for the system in which all information of actuator failures is known.

For each concurrent actuator failure case $k$ that has two failures with actuator failure indices $k_{1}$ and $k_{2}$, we have $\tau_{k_{1}}=\bar{\tau}_{k_{1}}, \tau_{k_{2}}=\bar{\tau}_{k_{2}}, \tau_{j}=\tau_{c j}^{*}$ for $j=1,2, \ldots, m$ with $j \neq k_{1}, k_{2}$ and $k_{1} \neq k_{2}$. The nominal control signal for each concurrent failure case is $\tau(t)=\tau_{c}^{*}(t)=\tau_{k(c o n)}^{*}(t)$ with $k=1,2, \ldots, \bar{k}$, where $\bar{k}$ is the number of all actuator failure pattern with two concurrent failures. The control signal $\tau_{k(\text { con })}^{*}(t)$ can be designed as

$$
\tau_{c}^{*}(t)=\tau_{k(c o n)}^{*}(t)=\left[\alpha_{1} \tau_{k a(c o n) 1}^{*}, \alpha_{2} \tau_{k a(c o n) 2}^{*}, \ldots, \alpha_{n} \tau_{k a(c o n) m}^{*}\right]^{T},
$$

where $\alpha_{i}=0$ if the actuator with the actuator index $i$ fails and $\alpha_{i}=1$ otherwise. 
Define the intermediate control signal $\tau_{a(c o n)}^{*}$ as a vector consisting of the non-zero rows of $\tau_{k(\text { con })}^{*}$. The control signal $\tau_{k a(\text { con })}^{*}$ can be chosen as

$$
\tau_{k a((\text { con }))}^{*}(t)=h_{k(c o n)} \tau_{k 0(\text { con })}^{*}(t)
$$

where the matrix $h_{k(\text { con })} \in R^{n \times n}$. The signal $\tau_{k 0(\text { con })}^{*}(t) \in R^{n \times 1}$ can be chosen to satisfy

$$
E_{k_{1}} \bar{\tau}_{k_{1}}+E_{k_{2}} \bar{\tau}_{k_{2}}+\bar{E}_{k(\mathrm{con})} h_{k(\mathrm{con})} \tau_{k 0(\mathrm{con})}^{*}(t)=Y \theta^{*}-K_{d} s(t)
$$

where $\bar{E}_{k(\text { con })}$ is defined as a submatrix of $E$ without the $k_{1}$ and $k_{2}$ rows. The signal $\tau_{k 0(\text { con })}^{*}(t)$ can be written as

$$
\tau_{k 0(\text { con })}^{*}(t)=K_{k(\text { con }) 1}\left(Y \theta^{*}-K_{d} s(t)\right)+K_{k(\text { con }) 2} \bar{\tau}_{k_{1}}+K_{k(\text { con }) 3} \bar{\tau}_{k_{2}},
$$

where $K_{k(\text { con }) 1}=\left(\bar{E}_{k(\text { con })} h_{k(\text { con })}\right)^{-1}, K_{k(\text { con }) 2}=-\left(\bar{E}_{k(\text { con })} h_{k(c o n)}\right)^{-1} E_{k_{1}}$ and $K_{k(c o n) 3}=$ $-\left(\bar{E}_{k(\text { con })} h_{k(\text { con })}\right)^{-1} E_{K_{2}}$. This design ensures $E \tau_{c}^{*}=Y \theta^{*}-K_{d} s(t)$ for each concurrent actuator failure case, where both actuators with actuator failure indices $k_{1}$ and $k_{2}$ fail at the same time. The concurrent design for two actuator failures can be combined with the adaptive actuator failure compensation scheme (6.27) to allow up to two concurrent actuator failures as

$$
\tau_{c}(t)=\tau_{c \chi(0)}(t)+\sum_{i=1}^{m} \tau_{c \chi(i)}(t)+\sum_{k=1}^{\bar{k}} \tau_{k \chi(c o n)}(t)
$$

where $\tau_{k \chi(c o n)}(t)$ are the estimates of $\chi_{k(\text { con })}^{*} \tau_{k(\text { con })}^{*}$ and $\chi_{k(\text { con })}$ is the indicator function for each concurrent actuator failure case, which is the indicator function.

The parameterization of $\tau_{k \chi(i)(c o n)}(t)$ can be done in the same way as in (6.30). With $\bar{\tau}_{k_{1}}(t)=\rho_{k 1(c o n)}^{* T} f_{a k 1}(t), \bar{\tau}_{k_{2}}(t)=\rho_{k 2(c o n)}^{* T} f_{a k 2}(t)$, the parameterized control signal 
component $\tau_{\text {ka }(c o n)}$ can be written as

$$
\begin{aligned}
& \tau_{k a \chi(c o n)}(t)=-\operatorname{diag}\left\{\chi_{k(\text { con }) 1}, \ldots, \chi_{k(\text { con }) n}\right\} h_{k(\text { con })} K_{k(\text { con }) 1} K_{d} s(t) \\
& +h_{k(\mathrm{con})} K_{k(\mathrm{con}) 1} Y \theta_{k(\mathrm{con})} \\
& +\left[\begin{array}{c}
\rho_{k 1(\mathrm{con})(1)}^{T} f_{a k 1}(t) \phi_{k 1(\mathrm{con}) 1} \\
\rho_{k 1(\mathrm{con})(2)}^{T} f_{a k 1}(t) \phi_{k 1(\mathrm{con}) 2} \\
\vdots \\
\rho_{k 1(\mathrm{con})(n)}^{T} f_{a k 1}(t) \phi_{k 1(\mathrm{con}) n}
\end{array}\right]+\left[\begin{array}{c}
\rho_{k 2(\mathrm{con})(1)}^{T} f_{a k 2}(t) \phi_{k 2(\mathrm{con}) 1} \\
\rho_{k 2(\mathrm{con})(2)}^{T} f_{a k 2}(t) \phi_{k 2(\mathrm{con}) 2} \\
\vdots \\
\rho_{k 2(\mathrm{con})(n)}^{T} f_{a k 2}(t) \phi_{k 2(\mathrm{con}) n}
\end{array}\right]
\end{aligned}
$$

where $\theta_{k(c o n)}$ is the estimate of $\chi_{k(c o n)}^{*} \theta^{*}$ and $\chi_{k(c o n) j}$ is the estimate of $\chi_{k(c o n) j}^{*}$ for $j=1,2, \ldots, n$. The terms $\chi_{k(c o n) j}^{*} \rho_{k l(c o n)}^{*}$ is estimated by $\rho_{k l(\text { con })(j)}$ for $j=1,2, \ldots, n$ and $l=1,2$ with $\phi_{k 1(\text { con })}=\left[\phi_{k 1(c o n) 1}, \phi_{k 1(c o n) 2}, \ldots, \phi_{k 1(\text { con }) n}\right]^{T}=h_{k(c o n)} K_{k(c o n) 2}$ and $\phi_{k 2(\mathrm{con})}=\left[\phi_{k 2(\mathrm{con}) 1}, \phi_{k 2(\mathrm{con}) 2}, \ldots, \phi_{k 2(\mathrm{con}) n}\right]^{T}=h_{k(\mathrm{con})} K_{k(\mathrm{con}) 3}$. With the proposed parameterization scheme and the similar adaptive laws as in Section 4.3 can be applied to the adaptive actuator failure compensation scheme to update the estimated parameters $\theta_{k(\text { con })}, \chi_{k(c o n) j}$ and $\rho_{k l(c o n(j))}$ for $j=1,2, \ldots, n, k=1,2, \ldots, \bar{k}$ and $l=1,2$ to ensure the desired closed-loop stability and asymptotic output tracking.

Remark 6.3: To extend the proof of Theorem 6.1 to allow up to two concurrent actuator failures in the system, we need to consider additional Lypunov functions for the concurrent actuator failure pattern. In the previous proof, we have found multiple Lyapunov function for the all time intervals $\left(T_{i}, T_{i+1}\right), i=0,1,2, \ldots, n$ with $T_{0}=0, T_{n}=\infty$ on which up to one actuator failure occurs and the actuator failure pattern is fixed. To guarantee the system performance for all time, we need to find additional Lyapunov functions for all $\left(T_{i}, T_{i+1}\right)$ on which two concurrent actuator failures occur. For each concurrent actuator failure pattern $k$ for $k=1,2, \ldots, \bar{k}$ with 
two actuator failure, which the actuator failure indices are $k_{1}, k_{2}, k_{1} \neq k_{2}$, we consider the following Lyapunov function candidate $V_{k(c o n)}$

$$
\begin{gathered}
V_{k(\text { con })}=\frac{1}{2} s^{T} E^{T} D_{c} E s+\frac{1}{2} \sum_{i=0}^{m} \sum_{j=1}^{n} \tilde{\chi}_{i j}^{2} \gamma_{i j}^{-1}+\frac{1}{2} \sum_{i=0}^{m} \sum_{j=1}^{m} \tilde{\theta}_{i j}^{T} \beta_{i j}^{-1} \tilde{\theta}_{i j} \\
+\frac{1}{2} \sum_{i=1}^{m} \sum_{j=1}^{n} \tilde{\rho}_{i(j)}^{T} \Gamma_{i j}^{-1} \tilde{\rho}_{i(j)}-\frac{1}{2} \sum_{i=0}^{m} \tilde{\chi}_{i k_{1}}^{2} \gamma_{i k_{1}}^{-1}-\frac{1}{2} \sum_{i=0}^{m} \tilde{\theta}_{i k_{1}}^{T} \beta_{i k_{1}}^{-1} \tilde{\theta}_{i k_{1}} \\
-\frac{1}{2} \sum_{i=1}^{m} \tilde{\rho}_{i\left(k_{1}\right)}^{T} \Gamma_{i k_{1}}^{-1} \tilde{\rho}_{i\left(k_{1}\right)}-\frac{1}{2} \sum_{i=0}^{m} \tilde{\chi}_{i k_{2}}^{2} \gamma_{i k_{2}}^{-1} \\
-\frac{1}{2} \sum_{i=0}^{m} \tilde{\theta}_{i k_{2}}^{T} \beta_{i k_{2}}^{-1} \tilde{\theta}_{i k_{2}}-\frac{1}{2} \sum_{i=1}^{m} \tilde{\rho}_{i\left(k_{2}\right)}^{T} \Gamma_{i k_{2}}^{-1} \tilde{\rho}_{i\left(k_{2}\right)}
\end{gathered}
$$

The time derivative of the Lyapunov function $V_{k(c o n)}$ is

$$
\dot{V}_{k(\mathrm{con})} \leq-s^{T} K_{d} s \leq 0
$$

With the additional Lyapunov function, we have considered all possible failure cases of up to two concurrent actuator failures for all time intervals $\left(T_{0}, T_{n}\right)$. Similarly to the proof shown in Theorem 6.1, we can show that $s(t) \in L^{\infty} \bigcap L^{2}$ and all signals in the system are bounded, based on the Barbalat lemma we can conclude that the error vector $\lim _{t \rightarrow \infty} s(t)=0$. Thus, the adaptive actuator failure compensation scheme can guarantee the asymptotic tracking property for the system with parameter uncertainties subjected to uncertain actuator failures. With the additional design of the concurrent actuator failure case, the proposed algorithm allows up to two actuators to fail at the same time. The similar approach could also be used to extend the developed algorithm to cases when more than two actuators fail at the same time.

Remark 6.4: It is possible for the adaptive actuator failure compensation scheme to handle a case of a square wave actuator failure signal. There are two approaches to solve this problem as follows: 
1. If the time instance that the actuator failure occurs is unknown and the period of the square wave signal is relatively large, we can treat the failure similar to the constant actuator failure case. This approach could stabilize the system and the tracking performance is acceptable; however, we cannot guarantee asymptotic tracking because the controller needs to adapt at each edge of the square wave failure signal.

2. In order to achieve asymptotic tracking, we need to model the square wave failure signal. From the actuator failure model (6.3), the base-function of the complete square wave signal can be described as

$$
f_{a i}(t)=\operatorname{sgn}\left[\frac{2 \pi\left(t-t_{f}\right)}{T}\right],
$$

where $T$ represents the period of the square wave and $t_{f}$ is the time that actuator failure occurs. However, this basic square wave function can only represent the most basic form of a square wave signal. In order to handle multiple types of square wave signal, we model the failure with two base-function as

$$
\begin{aligned}
\bar{\tau}_{i}= & \bar{\tau}_{i 1} f_{a 1}(t)+\bar{\tau}_{i 2} f_{a 2}(t), \\
f_{a 1}(t)= & \frac{\operatorname{sgn}\left[\frac{2 \pi\left(t-t_{f 1}\right)}{T_{1}}\right]+1}{2}, f_{a 2}(t)=\frac{\operatorname{sgn}\left[\frac{2 \pi\left(t-t_{f 2}\right)}{T_{2}}\right]-1}{2} .
\end{aligned}
$$

This allows multiple types of square wave failure signal such a square wave with unequal period or different starting wave. With the proposed actuator failure model, we can use our adaptive actuator failure compensation scheme to handle a system with possible square wave actuator failure signal. This approach can also guarantee the asymptotic tracking of the system with parameter uncertainties. 


\subsection{Simulations Study}

In order to demonstrate the effectiveness of the proposed control scheme, we consider a two-dimensional cooperative manipulator system as a benchmark system in our study. The system contains two manipulators, which are attached to each side of a rigid platform as shown in Figure 6.3. The benchmark example that we choose is capable of representing a cooperative manipulator system in general; Additionally, the benchmark system is relatively easy to understand, which make the it a good example to use for demonstrating the effectiveness of our control algorithm.

As in Figure 6.3, the system uses three actuators $q_{1}, q_{2}, q_{3}$ to support a rigid platform. The actuator $q_{2}$ is added to increase redundancy in the system to compensate for the possible failure that could occurs on the left side of the platform.

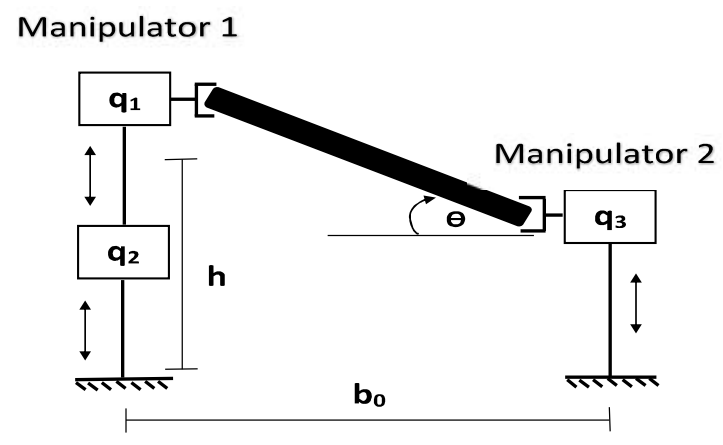

Figure 6.1: The benchmark cooperative manipulator system.

\subsubsection{The Benchmark System Model}

In this study, we derive the dynamic model of the benchmark system using the EulerLagrange equation. Based on the generalized coordinate $l_{i}$ for $i=1,2,3$, we consider Lagrange's equation

$$
\frac{d}{d t}\left(\frac{\partial L}{\partial \dot{l}_{i}}\right)-\frac{\partial L}{\partial l_{i}}=\tau_{i}
$$


where the Lagrangian $L=T-V$, $\mathrm{T}$ is the kinetic energy, $\mathrm{V}$ is the potential energy, $l_{i}=\left[l_{1}, l_{2}, l_{3}\right]$ are the vectors of generalized coordinates that represent the length of each actuator, and $\tau_{i}$ is the torque of each actuator, which acts along each coordinate. For simplicity, we assume that the dependency forces between $q_{1}, q_{2}$ is relatively small. The kinetic energy and the potential energy can be written as

$$
\begin{aligned}
& T=\frac{1}{2}\left(m_{1} \dot{l}_{1}^{2}+\left(m_{1}+m_{2}\right) \dot{l}_{2}^{2}+m_{3} \dot{l}_{3}^{2}\right)+\frac{1}{2} m_{p} \dot{h}^{2}+\frac{1}{2} I_{p} \dot{\theta}^{2} \\
& V=m_{1} g l_{1}+\left(m_{1}+m_{2}\right) g l_{2}+m_{3} g l_{3}+m_{p} g h,
\end{aligned}
$$

where $\mathrm{g}$ is the scalar value of the gravity, $m_{i}=1 \mathrm{~kg}$ is the mass of each actuator. With the small angle approximation technique, the Lagrange's equation can be written as

$$
\begin{aligned}
& \tau_{1}=\ddot{l}_{1}+\frac{m_{p}}{2}\left(\ddot{l}_{1}+\ddot{l}_{2}+\ddot{l}_{3}\right)+\frac{I_{p}}{b_{o}}\left(\ddot{l}_{1}+\ddot{l}_{2}-\ddot{l}_{3}\right)+\left(1+\frac{1}{2} m_{p}\right) g \\
& \tau_{2}=2 \ddot{l}_{2}+\frac{m_{p}}{2}\left(\ddot{l}_{1}+\ddot{l}_{2}+\ddot{l}_{3}\right)+\frac{I_{p}}{b_{o}}\left(\ddot{l}_{1}+\ddot{l}_{2}-\ddot{l}_{3}\right)+\left(2+\frac{1}{2} m_{p}\right) g \\
& \tau_{3}=\ddot{l}_{3}+\frac{m_{p}}{2}\left(\ddot{l}_{1}+\ddot{l}_{2}+\ddot{l}_{3}\right)-\frac{I_{p}}{b_{o}}\left(\ddot{l}_{1}+\ddot{l}_{2}-\ddot{l}_{3}\right)+\left(1+\frac{1}{2} m_{p}\right) g .
\end{aligned}
$$

Because the height and the orientation of the system can be specified using by the length of each platform. In this study, we let $x_{o 1}=\left[l_{1}, l_{2}\right]$ and $x_{o 2}=l_{3}$. With the dynamic model of the system from the Lagrange's equation, we could transform the system into a general form to use in the simulation of the adaptive actuator failure compensation scheme in the next subsection.

\subsubsection{Benchmark System in General Form}

In this subsection, we define $x_{o}=\left[x_{o 1}, x_{o 2}\right]^{T}$ as state of out system which could be used to specific the height and orientation of the platform and $\tau_{i}$ for $i=1,2,3$ is the force that each actuator $q_{i}$ produces. With the mass of each actuator is assigned as 
one, the dynamic of the system can be written in the general form as

$$
D_{c}\left(x_{o}\right) \ddot{x}_{o}+C_{c}\left(x_{o}, \dot{x}_{o}\right) \dot{x}_{o}+G_{c}\left(x_{o}\right)=E\left(x_{o}\right) \tau \text {. }
$$

The matrices $D_{c}, C_{c}, G_{c}$ and $E$ can be written as

$$
\begin{gathered}
D_{c}=\left[\begin{array}{cc}
2+\frac{3 m_{p}}{2}+\frac{3 I_{p}}{b_{o}} & \frac{m_{p}}{2}-\frac{I_{p}}{b_{o}} \\
\frac{m_{p}}{2}-\frac{I_{p}}{b_{o}} & 1+\frac{m_{p}}{2}+\frac{I_{p}}{b_{o}}
\end{array}\right], C_{c}=\left[\begin{array}{ll}
0 & 0 \\
0 & 0
\end{array}\right] \\
G_{c}=\left[\begin{array}{c}
4 g+\frac{3 m_{p} g}{2} \\
g+\frac{m_{p} g}{2}
\end{array}\right], E=\left[\begin{array}{lll}
2 & 1 & 0 \\
0 & 0 & 1
\end{array}\right],
\end{gathered}
$$

where $g$ is the gravitational constant, $m_{p}$ is the mass of the platform and $I_{p}$ is in the moment of inertia of the platform. We can see that this system has redundancy that allows either actuator $q_{1}$ or $q_{2}$ to fail. If actuator $q_{3}$ fails, the rank of submatrix $\bar{E}_{(3)}$ becomes one, which violates Assumption 1, so we will not consider this case in the simulation study.

\subsubsection{Simulation Conditions}

In the simulation study, we simulate the system using the Matlab software with ode45 function. In order to see the performance of the algorithm, we choose the gains in the system to be relatively small with $K_{d}=\left[\begin{array}{lll}5 & 0,0 & 5\end{array}\right]$ and all adaptive gain is chosen to be 0.1 . We assign the parameter of the system as $m_{p}=10 \mathrm{~kg}, I_{p}=1 \mathrm{~kg} \cdot \mathrm{m}^{2}$ and $b_{0}=1 \mathrm{~m}$. These parameters are unknown to the controller.

The initial conditions of the system are chosen as $x_{o}=[1,1]^{T}$, the reference signal is chosen as $x_{d}(t)=[1+0.1 \sin (0.1 t), 1+0.1 \sin (0.1 t)]^{T}$. Although the adaptive actuator failure compensation developed in this study can handle most initial values of 
the parameters in the system, we need to be careful when choosing some initial values to prevent matrix singularity. For example, we cannot choose the initial value of the mass of the system to be zero. For simplicity, in this study the initial parameters of the system are chosen to be one.

In this simulation study, two simulations with different types of failure are evaluated as follows.

Lock in-place failure of the actuator. In this simulation, we use constant actuator failure signals. We let actuator failure occurs twice as follows:

(i) No actuator failure case: $\tau(t)=\tau_{c}(t)$ for $0 \leq t<50 \mathrm{~s}$,

(ii) Actuator $q_{1}$ failure case: $\tau_{1}=\bar{\tau}_{1}=20$ at $50 \leq t<100 s$,

(iii) The failing actuator $q_{1}$ becomes normal again, no actuator failure case: $\tau(t)=$ $\tau_{c}(t)$ for $100 \leq t<150 s$, (iv) Actuator $q_{2}$ failure case: $\tau_{2}=\bar{\tau}_{2}=-30$ at $150 \leq t<200 s$.

Square wave actuator failure. In this case, we use square wave actuator failure signal. In order to demonstrate the effectiveness of our design, we study two cases of actuator failure with a different algorithm.

(i) Using original design by estimating the square wave actuator failure as a constant. In this case, we let the actuator failure occur at time $t=50 \mathrm{~s}$. The square wave failure signal is chosen as

$$
f_{a}(t)=5 * \operatorname{sgn}\left[\frac{2 \pi t}{10}\right] .
$$

(ii) In the second case, we introduce the square wave based function as introduce in Remark 6.2. We also let the same square wave actuator failure occur at time $t=50 \mathrm{~s}$. 


\subsubsection{Simulation Results}

For the lock in-place failure case, the simulation results show the output of the system $x_{o}$ versus the desirable trajectory $x_{d}$ in Figure 6.2. In this study, we assume that some parameters in the system are unknown. The estimated parameters $\hat{\theta}_{0}$, are shown in Figure 6.3. For the square wave actuator failure case, the simulation results of the first case are shown by Figure 6.4 and Figure 6.5 and the second case is shown by Figure 6.6 and Figure 6.7.
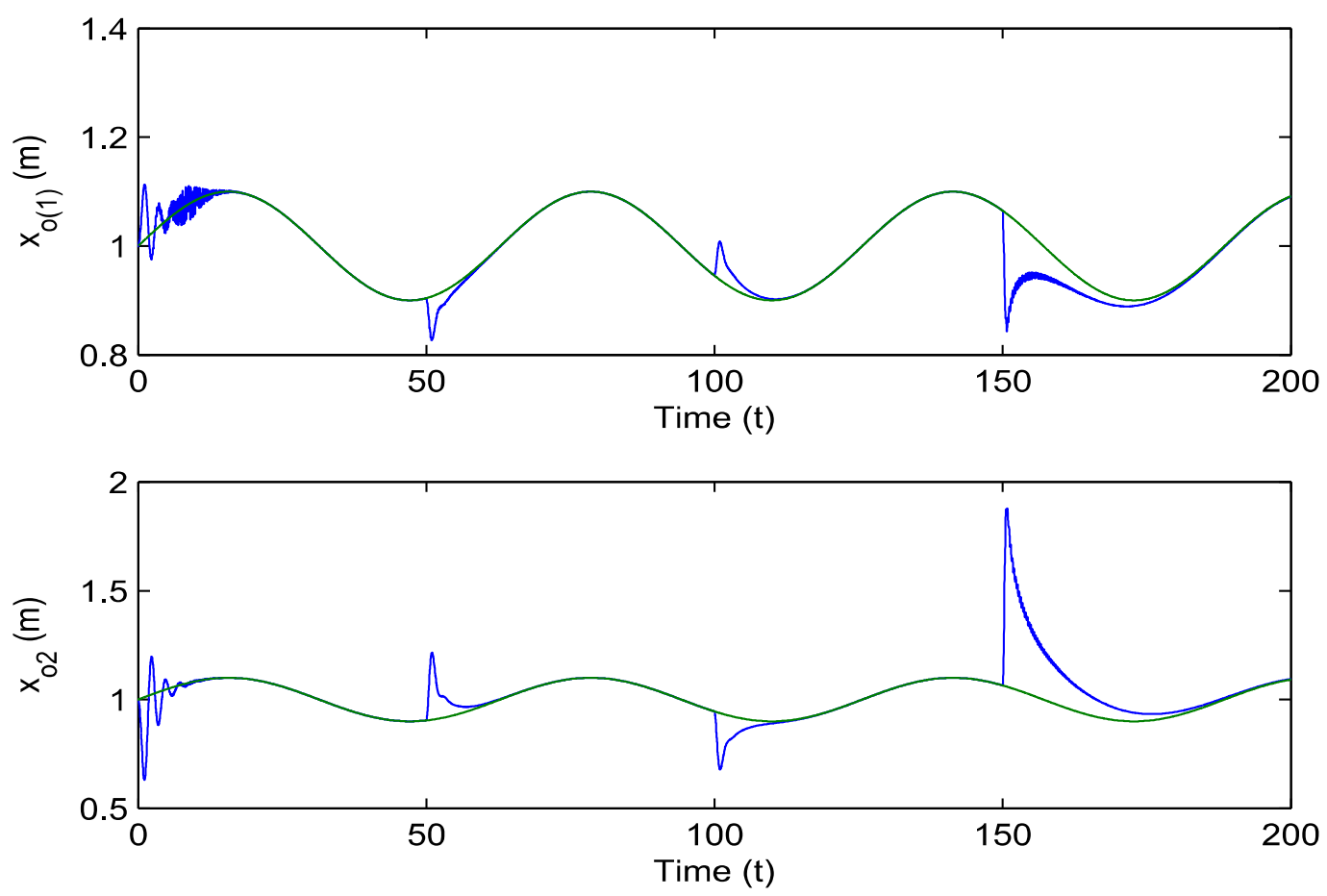

Figure 6.2: System output $x_{o}(t)$ vs. $x_{d}(t)$ for the lock in-place failure.

As we can see from Figure 6.2, the tracking error of the system always goes to zero. The simulation results confirm that the adaptive actuator failure compensation scheme can guarantee that the tracking error of the system goes to zero for the desired sinusoidal trajectory. From Figure 6.3, the study also shown that the estimated parameters are bounded but do not necessarily converge to the true value, which 
CHAPTER 6. DESIGN FOR A COOPERATIVE SYSTEM WITH PARAMETER
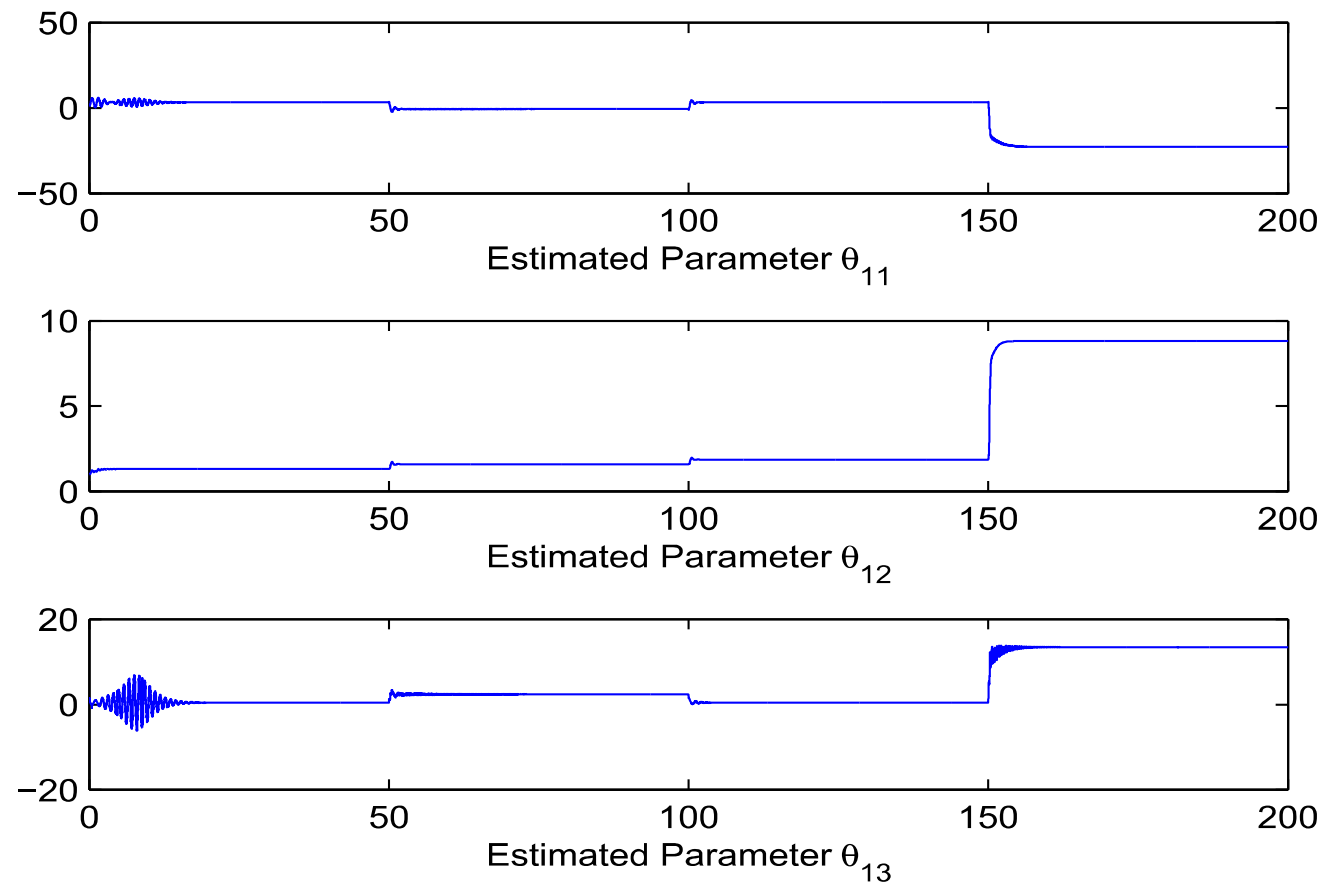

Figure 6.3: Estimated parameters $\hat{\theta}_{1}$ for the lock in-place failure.

is consistent with our theory and is acceptable from the control perspective. The adaptive actuator failure compensation scheme for the benchmark system allows $q_{1}$ or $q_{2}$ to fail, but both actuators cannot fail at the same time. 

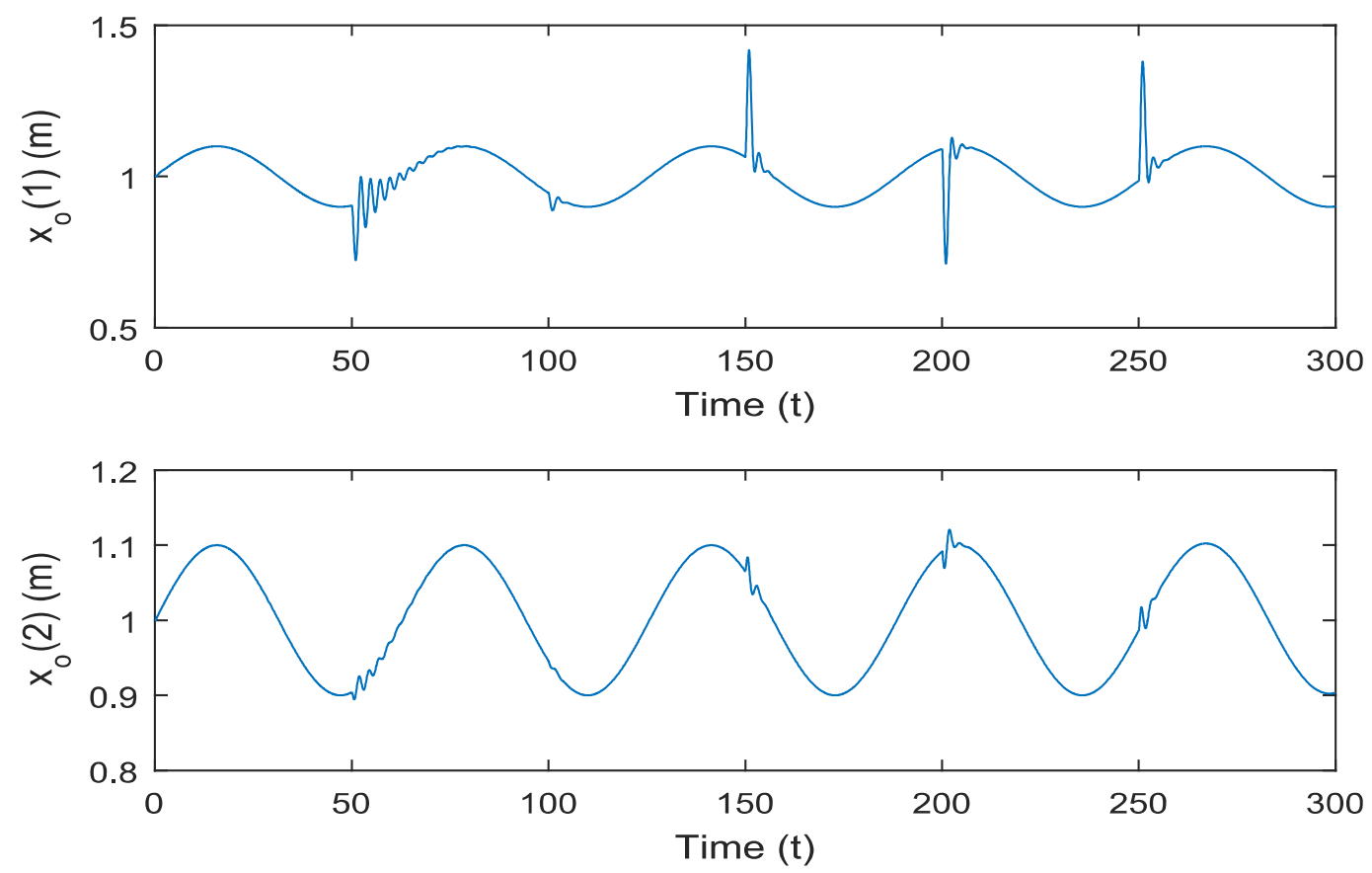

Figure 6.4: Estimated parameters $\hat{\theta}_{1}$ for the square wave actuator failure.
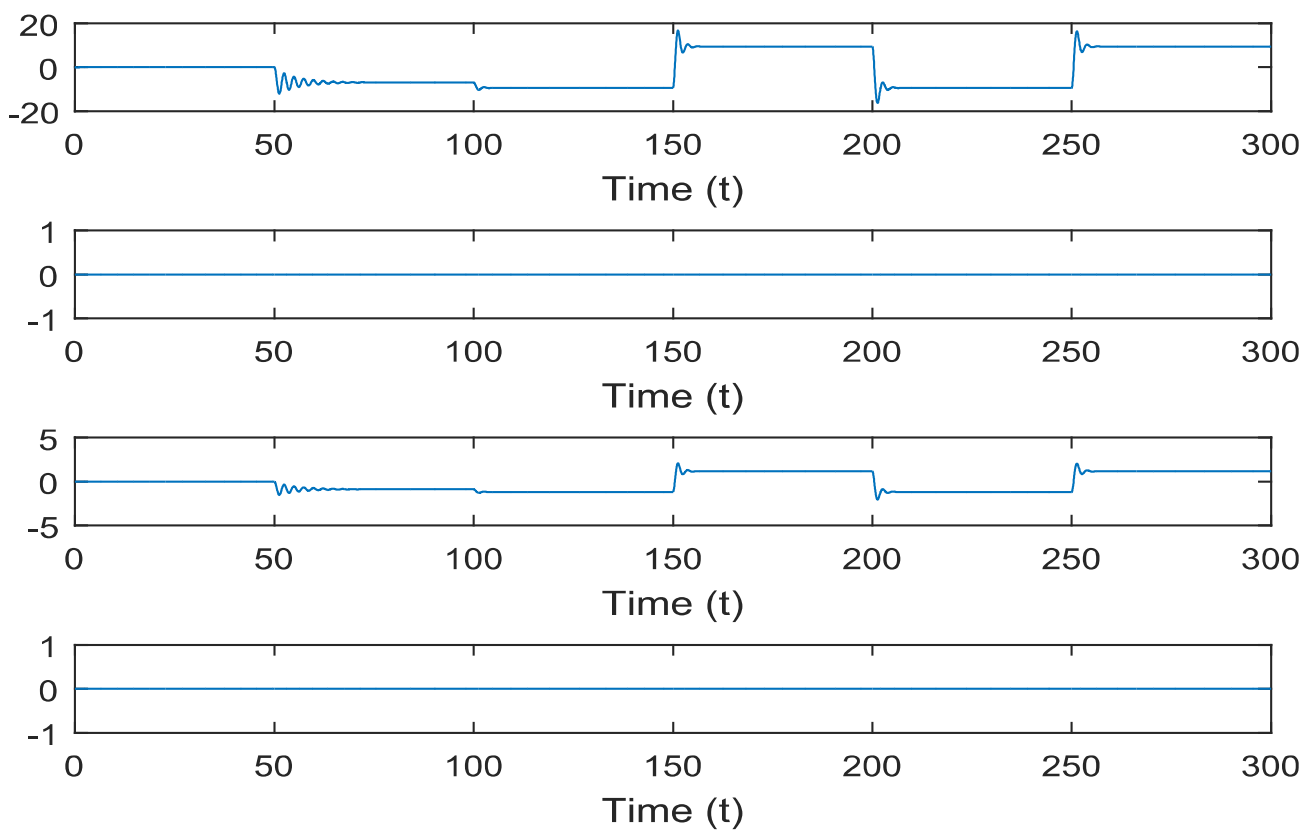

Figure 6.5: Estimated actuator failure signal $\rho_{1(1)}, \rho_{1(2)}, \rho_{2(1)}$ and $\rho_{2(2)}$. 
From Figure 6.4 and Figure 6.5, we can see that it is possible to stabilize the system by estimated the square wave actuator failure signal as a constant. However, every time the square wave signal changes its magnitude, it will take some time for the controller to adapt to the changes.
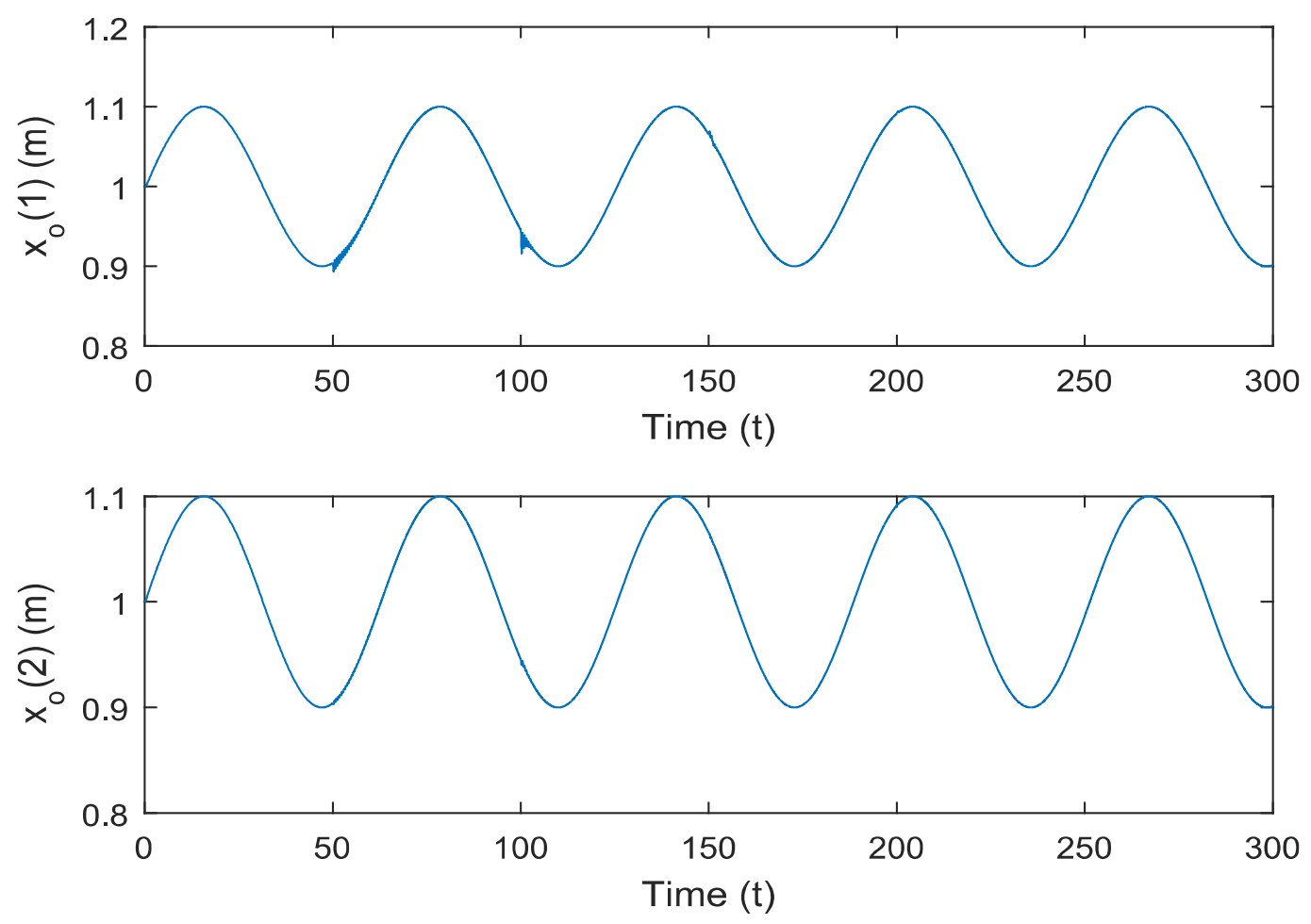

Figure 6.6: System output $x_{o}(t)$ vs. $x_{d}(t)$ for the square wave actuator failure. 

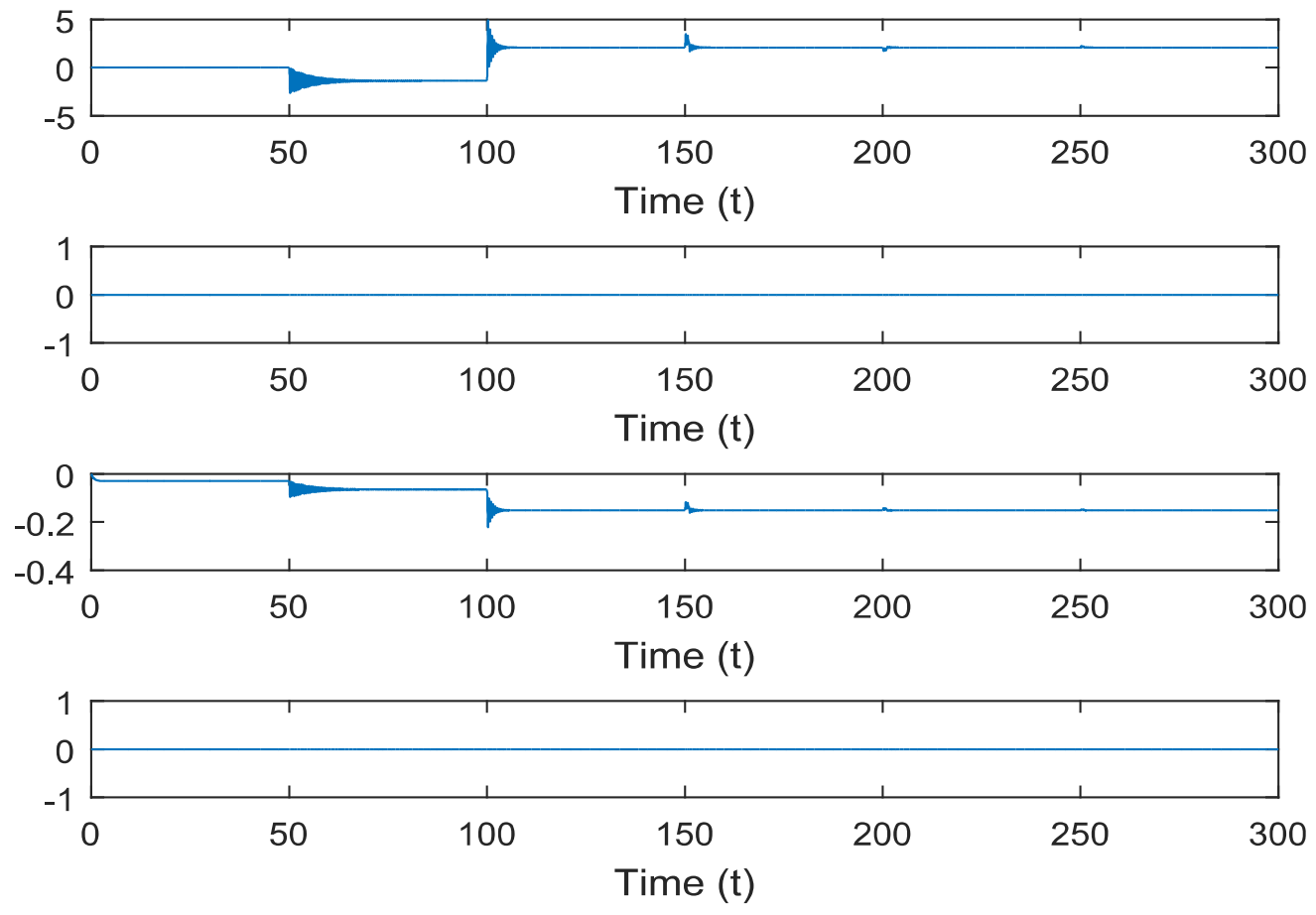

Figure 6.7: Estimated actuator failure signal $\rho_{1(1)}, \rho_{1(2)}, \rho_{2(1)}$ and $\rho_{2(2)}$.

By introducing the square wave based function to our algorithm, the simulation result in Figure 6.6 shows that the performance of the system is significantly improved compared to the original version in Figure 6.4. In this case, it is possible to guarantee that the tracking error of the system goes to zero for the desired sinusoidal trajectory. From the simulation result shown in Figure 6.7, we can see that the estimation schemes become more accurate and the tracking error of the system with square wave actuator failure is reduced over time, which confirm the performance of the adaptive actuator failure compensation scheme developed in this study. 


\subsection{Conclusions}

A cooperative manipulator robotic system plays an important role in many projects.

Developing an adaptive actuator failure compensation scheme that can immediately compensate for any possible failures in the system is important for the control and robotic fields. Since the cooperative manipulator systems often have redundancy, we can design a controller that utilizes the redundancy to control a cooperative manipulator system when actuator failures occur.

In this chapter, we have studied the adaptive actuator failure compensation scheme for a cooperative manipulator robotic system with parameter uncertainties in addition to actuator failure. The adaptive control design uses an integration of multiple individual failure compensators and direct adaptation to handle actuator failure and parameter uncertainties in the system. With complete proof and performance analysis, the proposed algorithm guarantees a desirable closed-loop stability and asymptotic tracking property can be achieved, despite uncertain actuator failures. The simulation results verified the performance of the proposed algorithm when applied to the cooperative manipulator system subjected to uncertain actuator failures, which included constant actuator failure as well as square wave actuator failure signal. 


\section{Chapter 7}

\section{Design for A Parallel Manipulator with Parameter Uncertainties}

A parallel manipulator is a mechanical system that uses several computer-controlled serial chains to support a single platform, or end-effector. With parallel manipulators, a high rigidity may be obtained with a small mass of the manipulator, which allows high precision with high speed of movements, and the redundancy of the actuator can be used to solve the actuator failure problem.

In this chapter, we develop a new adaptive actuator failure compensation scheme for a parallel robotic system with parameter uncertainties. The objective of the control design is to ensure the desired closed-loop stability and asymptotic output tracking of the system subject to uncertain actuator failure. This study makes the following contributions to the robotics and control fields:

- Develop an adaptive actuator failure compensation scheme for a parallel manipulator system with parameter uncertainties, in addition to actuator failure uncertainties.

- Study the dynamic and control of parallel structure robotic manipulator and demonstrate how to deal with the constraint of the parallel structure.

- Clarification of several key technical issues and solutions for the adaptive actu- 
ator failure compensation scheme for parallel manipulator system.

- Study the simulation of the adaptive actuator failure compensation scheme based on a 2-DOF redundantly actuated parallel manipulator subject to uncertain actuator failure.

The chapter is organized as follows. In Section 2, we formulate the problem with the discussion of the dynamic model of the parallel manipulator system, actuator redundancy, actuator failure model and control objective. In Section 3, we design a nominal controller by assuming that the knowledge of actuator failures is known. In Section 4, the adaptive control scheme is developed based on the nominal controller structure. Finally, in Section 5, we study the simulation of a redundantly actuated parallel manipulator to confirm the effectiveness of the control design.

\subsection{Problem Formulation}

The objective of this research is to develop an adaptive actuator failure compensation scheme to solve the actuator failure problem in robotic system. In this study, we utilize the redundancy in the structure of a parallel manipulator to ensure closed-loop stability and asymptotic output tracking of the system with parameter uncertainties in the presence of uncertain actuator failures.

\subsubsection{Dynamic Model of the Parallel Robots}

One of the most important problems, which makes parallel manipulators difficult to control, is the constraints in the parallel structure of the system. In this study, we will develop an adaptive actuator failure compensation scheme for a parallel robot with constraints. In this case, we consider a 2-DOF redundantly actuated parallel 
manipulator as our benchmark system. The parallel manipulator is actuated by three servo motors located at the bases A1, A2, and A3. The end-effector is mounted at the common joint $\mathrm{O}$, where the three manipulators meet. The joint angles are defined as follows: $q_{a 1}, q_{a 2}, q_{a 3}$ refer to the active joint angles, and $q_{b 1}, q_{b 2}, q_{b 3}$ refer to the passive joint angles.

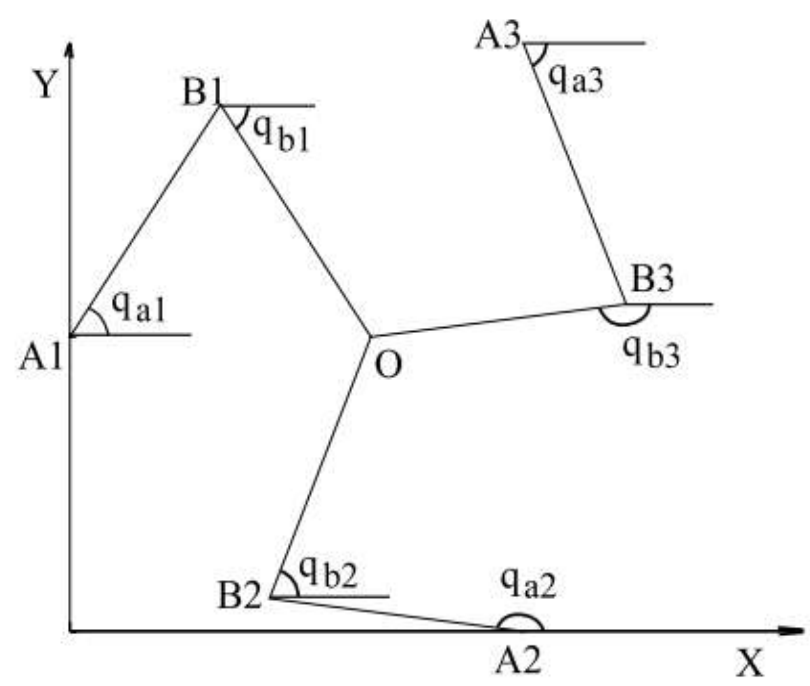

Figure 7.1: The 2-DOF redundantly actuated parallel manipulator.

The dynamic model of the parallel manipulator can be obtained by combining the dynamic model of each leg. According to [16], the dynamic model of each leg is

$$
D_{i} \ddot{q}_{i}+C_{i} \dot{q}_{i}+f_{i}=\tau_{i},
$$

where $\ddot{q}_{i}$ and $\dot{q}_{i}$ are the joint angular acceleration and angular velocity of each manipulator for $i=1,2,3, D_{i} \in R^{2 \times 2}$ is inertia matrix, and $C_{i} \in R^{2 \times 2}$ is Coriolis and centrifugal term. Also, $\tau_{i}=\left[\begin{array}{ll}\tau_{a i} & 0\end{array}\right]^{T}$ is joint torque vector with $\tau_{a i}$ as the actuated joint torque and the passive joint torque is zero, the friction torque vector is denoted as $f_{i}=\left[\begin{array}{ll}f_{a i} & 0\end{array}\right]^{T}$ as we assume that passive joint friction torque is negligible. The 
actuated joint friction torque $f_{a i}$ can be modeled as [16]

$$
f_{a i}=\operatorname{sign}\left(\dot{q}_{a i}\right) f_{c i}+f_{v i} \dot{q}_{a i}
$$

where $f_{c i}$ represents the Coulomb friction, and $f_{v i}$ represents the coefficient of the viscous friction.

The combined dynamic of the system with the closed-loop constraints can be expressed in the joint space as

$$
D_{c} \ddot{q}+C_{c} \dot{q}+f=\tau+A^{T} \lambda,
$$

where $D_{c} \in R^{6 \times 6}$ is the combined inertia matrix, $C_{c} \in R^{6 \times 6}$ is the combined Coriolis and centrifugal matrix, $\tau=\left[\tau_{a 1}, \tau_{a 2}, \tau_{a 3}, 0,0,0\right]^{T}$, is the torque vector, $f=$ $\left[f_{a 1}, f_{a 2}, f_{a 3}, 0,0,0\right]^{T}$, is the friction vector and the vector $q=\left[q_{a 1}, q_{a 2}, q_{a 3}, q_{b 1}, q_{b 2}, q_{b 3}\right]^{T}$ represents the angle of each manipulator joint in the system.

One of the difficult problems, which arises in the robotic system with parallel structure, is how to deal with the constraints. The constrained torque in the system is very difficult to measure; as a result, we cannot directly develop a controller that addresses this issue. In this study, we first consider the constraint force vector $A^{T} \lambda$, where the matrix $A$ is the differential of the closed-loop constrained equation $\frac{\partial H(q)}{\partial t}=$ $A(q) \dot{q}=0$ and $\lambda$ is a multiplier representing the magnitude of the constraint force.

Definition 7.1: Constraint: A constraint on a mechanical system is a relation of the form

$$
A(q) \dot{q}=0
$$

For a robotic system the matrix, we can derive the constraint matrix $A$ from a 
set of real value function $h_{i}(q), i=1,2,3,4$ that describe the closed-loop constraints of the parallel manipulator. In this case, we consider the constraint equations from the position of actuator $a 1$ to position of $a 2$ and from $a 1$ to $a 3$. Consider both $x$ and $y$ axis, we have

$$
H(q)=\left[\begin{array}{c}
h_{1} \\
h_{2} \\
h_{3} \\
h_{4}
\end{array}\right]=\left[\begin{array}{c}
x_{a 1}+l \cos \left(q_{a 1}\right)+l \cos \left(q_{b 1}\right)-x_{a 2}-l \cos \left(q_{a 1}\right)-l \cos \left(q_{b 2}\right) \\
y_{a 1}+l \sin \left(q_{a 1}\right)+l \sin \left(q_{b 1}\right)-y_{a 2}-l \cos \left(q_{a 1}\right)-l \sin \left(q_{b 2}\right) \\
x_{a 1}+l \cos \left(q_{a 1}\right)+l \cos \left(q_{b 1}\right)-x_{a 3}-l \cos \left(q_{a 3}\right)-l \cos \left(q_{b 3}\right) \\
y_{a 1}+l \sin \left(q_{a 1}\right)+l \sin \left(q_{b 1}\right)-y_{a 3}-l \cos \left(q_{a 3}\right)-l \sin \left(q_{b 3}\right)
\end{array}\right]=0,
$$

where $x_{a i}, y_{a i}$ represents the position of actuator $a i$ in the $x$ and $y$ axist respectively.

The matrix A can be obtain by differentiating the closed-loop constrained equation $\frac{\partial H(q)}{\partial t}=\frac{\partial H(q)}{\partial q} \dot{q}=A(q) \dot{q}=0$. We have $A(q)=\frac{\partial H(q)}{\partial q}$ such that $A(q) \dot{q}=0$ as defined in Definition 6.1 as.

$$
A=\left[\begin{array}{cccccc}
-l \sin \left(q_{a 1}\right) & l \sin \left(q_{a 2}\right) & 0 & -l \sin \left(q_{b 1}\right) & l \sin \left(q_{b 2}\right) & 0 \\
l \cos \left(q_{a 1}\right) & -l \cos \left(q_{a 2}\right) & 0 & l \cos \left(q_{b 1}\right) & -l \cos \left(q_{b 2}\right) & 0 \\
-l \sin \left(q_{a 1}\right) & 0 & l \sin \left(q_{a 3}\right) & -l \sin \left(q_{b 1}\right) & 0 & l \sin \left(q_{b 3}\right) \\
l \cos \left(q_{a 1}\right) & 0 & -l \cos \left(q_{a 3}\right) & l \cos \left(q_{b 1}\right) & 0 & -l \cos \left(q_{b 3}\right)
\end{array}\right]
$$

In order to deal with the unknown constraint force vector $A^{T} \lambda$, we define the Jacobian matrix $E \in R^{6 \times 2}$ such that $\dot{q}=E \dot{q}_{o}$ with $\dot{q}_{o}=\left[\begin{array}{ll}\dot{x} & \dot{y}\end{array}\right]^{T}$ represents the velocity of the end-effector. With the Jacobian matrix $E$ and the fact that $A(q) \dot{q}=0$, we have $A E \dot{q}_{o}=0$, from which one can conclude that the equation $E^{T} A^{T}=0$ holds. 
With this property, the dynamic model of the parallel manipulator with constraints can be written as

$$
E^{T} D_{c} \ddot{q}+E^{T} C_{c} \dot{q}+E^{T} f=E^{T} \tau
$$

The dynamic model can be rewritten in the task space by using the relation $\ddot{q}=\dot{E} \dot{q}_{o}+E \ddot{q}_{o}$ as

$$
E^{T} D_{c} E \ddot{q}_{o}+E^{T}\left(D_{c} \dot{E}+C_{c} E\right) \dot{q}_{o}+E^{T} f=E^{T} \tau
$$

Since we assume that the friction torque of the passive joints is zero, we can further simplify the dynamic model using a Jacobian matrix between the velocity of the end effector and the actuated joints as

$$
E^{T} D_{c} E \ddot{q}_{o}+E^{T}\left(D_{c} \dot{E}+C_{c} E\right) \dot{q}_{o}+J^{T} f_{a}=J^{T} \tau_{a},
$$

where $\tau_{a}$ is the torque of the actuated joints, $f_{a}$ is the friction of the actuated joint, and the Jacobian matrix $J \in R^{3 \times 2}$ is such that $E^{T} \tau=J^{T} \tau_{a}$.

Remark 7.1: In a robotic system, it is common to assume that the system satisfies the following structural properties:

a) $D$ is a symmetric and positive definite matrix.

b) $\dot{D}-2 C$ is a skew symmetric matrix.

With this assumption, it can be proven that the new dynamic model that we obtained (7.9) also satisfies the similar structural properties:

c) $E^{T} D_{c} E$ is a symmetric and positive definite matrix.

d) $\frac{\partial E^{T} D_{c} E}{\partial t}-2\left(E^{T}\left(D_{c} \dot{E}+C_{c} E\right)\right)$ is a skew symmetric matrix. 


\subsubsection{Actuator Redundancy}

In order to develop an actuator failure compensation scheme, having redundancy in the system is one of the most crucial requirements. A parallel manipulator robotic system usually satisfies this requirement and it is also relatively easy to increase the number of actuators in a parallel manipulator system; thus, the study of parallel structure in robotic systems could open a new way that can improve both the performance and reliability of a robotic system in the future.

The adaptive actuator failure compensation scheme developed in this study utilizes the redundancy from the parallel structure to compensate for possible actuator failures. In order to achieve this objective, a system with $n$ degrees of freedom needs at least $n$ functioning actuators in the system [25]. Thus, the 2-DOF redundantly actuated parallel manipulator needs at least 2 functioning actuators, which allows an actuator failure to occur. In order to guarantee that the solution of the adaptive actuator failure compensation exists, we consider a matrix $\bar{J}_{(i)}$ as a submatrix of $J$ in (7.9) without the associated column $J_{1}, J_{2}$ or $J_{3}$ corresponding to the actuator failure index of each actuator failure case $i=1,2,3$. In this case, we can see that the rank of the matrix $\bar{J}_{(i)}$ is always equal to 2 for every actuator failure pattern; thus, it is possible to develop an adaptive actuator failure compensation for this system, which allows an actuator failure to occur.

\subsubsection{Control Objective}

Our control objective is to guarantee the position of the end-effector of the 2-DOF redundantly actuated parallel manipulator, or $q_{o}(t)$ tracks a desirable trajectory $q_{d}(t)$ asymptotically even when an actuator failure occurs. Consider the parallel manipulator model (7.9), due to possible actuator failures, an applied feedback control signal 
$\tau_{c}(t)=\left[\tau_{c 1}, \tau_{c 2}, \tau_{c 3}\right]^{T}$ may not reach the system, as $\tau_{a i}(t)$ is not equal to $\tau_{c i}(t)$. More precisely, in the presence of failures, the signal $\tau_{a}(t)$ is

$$
\tau_{a}(t)=(I-\sigma(t)) \tau_{c}(t)+\sigma(t) \bar{\tau},
$$

where $\bar{\tau}$ is an unknown failure vector and $\sigma(t)=\operatorname{diag}\left\{\sigma_{1}, \sigma_{2}, \sigma_{3}\right\}$ is the actuator failure pattern matrix such that $\sigma_{i}(t)=1$ if the $i$ actuator fails and $\sigma_{i}(t)=0$ otherwise.

The control objective is to design a feedback control signal $\tau_{c}(t)$ such that the closed-loop system $E^{T} D_{c} E \dot{s}+E^{T}\left(D_{c} \dot{E}+C_{c} E\right) s+K_{d} s=0$, despite the uncertain actuator failures $\tau_{a}(t)=(I-\sigma(t)) \tau_{c}(t)+\sigma(t) \bar{\tau}$, where the error vector $s$ is defined as $s=\dot{e}+\Lambda_{0} e$ with $e=q_{o}-q_{d}, q_{d}$ is the desired trajectory of the end-effector, $\Lambda_{0} \in R^{2 \times 2}$ is a design matrix whose eigenvalues have positive real parts and the gain matrix $K_{d}$ is chosen to be positive definite. When this objective is met, the system will be ensured to have the desired signal boundedness and tracking properties.

\subsection{Nominal control design}

In this section, we develop a nominal controller for the 2-DOF redundantly actuated parallel manipulator by assuming that the knowledge of the actuator failures is known. Such a controller structure can be used to design an adaptive actuator failure compensation scheme with uncertain actuator failures in the following section.

\subsubsection{Based Line Control Approach}

Before we begin the development of the actuator failure compensation, we first study the conventional controller for the system, which is developed based on the Slotine and Li's approach [18]. Consider the dynamic model of the parallel manipulator 
system (7.9)

$$
E^{T} D_{c} E \ddot{q}_{o}+E^{T}\left(D_{c} \dot{E}+C_{c} E\right) \dot{q}_{o}+J^{T} f_{a}=J^{T} \tau_{a}
$$

In order to develop a controller for the system, we first define an error vector $s=\dot{e}+\Lambda_{0} e \in R^{2}$, where $e=q_{o}-q_{d}, v=\dot{q}_{d}-\Lambda_{0} e \in R^{2}$ being an intermediate vector signal and let $\Lambda_{0}$ be a constant matrix whose eigenvalues have positive real parts. The definition of this error vector means that the convergence of $s$ also implies the convergence of the tracking error. Since some parameters in $D_{c}, C_{c}$ and $f_{a}$ are unknown, we choose $\tau_{a}(t) \in R^{3}$ to meet

$$
J^{T} \tau_{a}=E^{T} \hat{D} E \dot{v}+E^{T}\left(\hat{D}_{c} \dot{E}+\hat{C}_{c} E\right) v+J^{T} \hat{f}-K_{d} s(t)
$$

where $\hat{D}, \hat{C}, \hat{f}$ are the estimates of $D_{c}, C_{c}, f_{a}$ respectively.

We can linearly parameterize the system by introducing the following parameterization.

$$
E^{T} D E \dot{v}+E^{T}\left(D_{c} \dot{E}+C_{c} E\right) v+J^{T} f=Y(q, \dot{q}, v, \dot{v}) \theta^{*}
$$

where $\theta^{*}$ being the unknown parameter vector in the system that is estimated by $\hat{\theta}$ and $Y(q, \dot{q}, v, \dot{v})$ being a known regressor. In robotic system, the parameter uncertainty is unstructured since it represents an unknown value such as the mass or the inertia in the system. 
With the known regressor matrix, we write

$$
E^{T} \hat{D} E \dot{v}+E^{T}\left(\hat{D}_{c} \dot{E}+\hat{C}_{c} E\right) v+J^{T} \hat{f}=Y \hat{\theta}-K_{d} s(t) .
$$

Define $\tilde{\theta}=\hat{\theta}-\theta^{*}$ as the parameter error, the closed-loop system becomes

$$
E^{T} \hat{D}_{c} E \dot{s}+E^{T}\left(\hat{D}_{c} \dot{E}+\hat{C}_{c} E\right) s+K_{d} s=Y \tilde{\theta}
$$

\subsubsection{Actuator Failure Compensation}

In this study, we consider the 2-DOF redundantly actuated parallel manipulator whose actuators are subject to an actuator failure (??), that is, to design a feedback control signal $\tau_{c}^{*}(t)$ to meet $J^{T} \tau_{a}(t)=Y(q, \dot{q}, v, \dot{v}) \theta^{*}-K_{d} s(t)$ for $\tau_{a}=(I-\sigma) \tau_{c}^{*}+\sigma \bar{\tau}$, so that $E^{T} D_{c} E \dot{s}+E^{T}\left(D_{c} \dot{E}+C_{c} E\right) s+K_{d} s=0$.

Our proposed control scheme chooses a nominal controller structure as a combination of the nominal controller of each actuator failure pattern. We first design multiple individual control schemes for each actuator failure case.

Design for the no failure case $\left(\tau_{a}(t)=\tau_{c}^{*}(t)\right)$ : We consider

$$
\tau_{c}^{*}(t)=\tau_{c(0)}^{*}(t)=h_{a} \tau_{c a(0)}^{*}(t)
$$

for some chosen matrix function $h_{a} \in R^{3 \times 3}$ and the signal $\tau_{c a(0)}^{*}(t) \in R^{3 \times 1}$ are determined from

$$
J^{T} h_{a}\left(q_{o}\right) \tau_{c a(0)}^{*}(t)=Y \theta^{*}-K_{d} s(t)
$$

We need to choose a matrix $h_{a}$ such that the function $\bar{J}_{(i)}^{T} h_{i}$ is invertible. For example, 
if the matrix $\bar{J}_{(i))}$ is a not square matrix, then we will need to choose $h_{i}$ such that $\bar{J}^{T} h_{a}$ is a square matrix and is invertible. Furthermore, the design matrix $h_{i}$ can also be used for the load distribution of actuators in the system.

With the assumption of actuator redundancy that we have made, it is possible to choose $h_{a}$, such that the solution of $\tau_{c a(0)}^{*}(t)$ exists. The explicit form of the $\tau_{c a(0)}^{*}(t)$ can be written as

$$
\tau_{c a(0)}^{*}(t)=K_{a 1}\left(Y \theta^{*}-K_{d} s(t)\right)
$$

for some $K_{a 1} \in R^{3 \times 2}$. Since the matrix $K_{a 1}$ has more rows than its columns, this structure of $K_{a i}$ admits possibility up to $m-n$ constrained optimality. For unique solution, in this study we choose the controller by using only two actuators to control the system. By setting $\tau_{c 1}=0$, the controller can be chosen as

$$
\tau_{c(0)}^{*}(t)=\left[\begin{array}{ll}
0 & \tau_{c b(0)}^{* T}(t)
\end{array}\right]^{T}, \tau_{c b(0)}^{*}(t)=h_{0} \tau_{c 0(0)}^{*}(t) .
$$

With the submatrix $\bar{J}_{(0)}=\left[J_{2}, J_{3}\right]$, we choose $h_{0}$ and $\tau_{c 0(0)}^{*}(t)$ as

$$
\bar{J}_{(0)}^{T} h_{0} \tau_{c 0(0)}^{*}(t)=Y \theta^{*}-K_{d} s(t)
$$

The explicit form of $\tau_{c 0(0)}^{*}(t)$ is

$$
\tau_{c 0(0)}^{*}(t)=K_{01}\left(Y \theta^{*}-K_{d} s(t)\right)
$$

where $K_{01}=\left(\bar{J}_{(0)}^{T} h_{0}\right)^{-1}$.

This design ensures $J^{T} \tau_{c}^{*}=Y \theta^{*}-K_{d} s(t)$ as desired. 
Design for one actuator failure case $\left(\tau_{i}=\bar{\tau}_{i}, \tau_{j}=\tau_{c j}^{*}, j=1,2,3, j \neq i\right)$ : We consider

$$
\tau_{c}^{*}(t)=\tau_{c(i)}^{*}(t)=\left[\alpha_{1} \tau_{c a(i) 1}^{*}, \alpha_{2} \tau_{c a(i) 2}^{*}, \alpha_{3} \tau_{c a(i) n}^{*}\right]^{T},
$$

where $\alpha_{i}=0$ if the actuator $i$ fails and $\alpha_{i}=1$ otherwise. Defining the vector signal $\tau_{c a(i)}^{*}$ as a vector consisting of the non-zero rows of $\tau_{c}^{*}$, we consider

$$
\tau_{c a(i)}^{*}(t)=h_{i} \tau_{c 0(i)}^{*}(t)
$$

with $h_{i} \in R^{2 \times 2}$ and $\tau_{c 0(i)}^{*}(t) \in R^{2 \times 1}$ are obtained from

$$
J_{i}^{T} \bar{\tau}+\bar{J}_{(i)}^{T} h_{i} \tau_{c 0(i)}^{*}(t)=Y \theta^{*}-K_{d} s(t),
$$

where the submatrix $\bar{J}_{(i)} \in R^{2 \times 2}$ represents the Jacobian matrix $E$ without the associated $i$ row.

The explicit form of $\tau_{c 0(i)}^{*}(t)$ can be written as

$$
\tau_{c 0(i)}^{*}(t)=K_{i 1}\left(Y \theta^{*}-K_{d} s(t)\right)+K_{i 2} \bar{\tau}_{i} .
$$

where $K_{i 1} \in R^{2 \times 2}, K_{i 2} \in R^{2 \times 1}$ are expressed as

$$
K_{i 1}=\left(\bar{J}_{(i)}^{T} h_{i}\right)^{-1}, K_{i 2}=-\left(\bar{J}_{(i)}^{T} h_{i}\right)^{-1} J_{i} .
$$

This ensures $J^{T} \tau=J^{T}(I-\sigma) \tau_{c}^{*}+J^{T} \sigma \bar{\tau}=Y \theta^{*}-K_{d} s(t)$ for each actuator failure case.

Composite control design. With the control structure for the no actuator 
failure case and three cases of one actuator failure case, we can design a composite control law for the system by combining all cases as

$$
\tau_{c}^{*}(t)=\sum_{i=0}^{3} \chi_{i}^{*} \tau_{c(i)}^{*}(t)
$$

where $\chi_{i}^{*}$ for $i=0,1,2,3$ are the indicator functions of the actuator failure: $\chi_{0}^{*}=1$ when there is no actuator failure, $\chi_{i}^{*}=1$ for $i$ actuator failure. The indicator functions $\chi_{i}^{*}=0$ for its non-corresponding cases.

With the knowledge of the indicator functions and the nominal control signals, this design ensures $J^{T}(\theta) \tau=Y \theta^{*}-K_{d} s(t)$ for every case of actuator failure. In practice it is very difficult to obtain the knowledge of which actuator has failed, in the next section we will develop an adaptive actuator failure compensation scheme without the knowledge of which actuator has failed.

\subsection{Adaptive Failure Compensation Design}

The nominal control design derived in the previous section needs the knowledge of the actuator failures as well as the values of every parameter in the system, which may not be possible to measure. In this section, we develop an adaptive actuator failure compensation scheme for the parallel manipulator system with unknown parameters in the presence of uncertain actuator failures.

The system block diagram of the adaptive actuator failure compensation scheme is shown in Figure 7.2. The control input $\tau_{c}(t)$ is the combination of each control scheme based on the nominal controller from the previous section, which each controller is developed using the Slotine and Li's approach. There are estimator corresponding to each controller, the estimators estimate the actuator failure values, actuator failure 


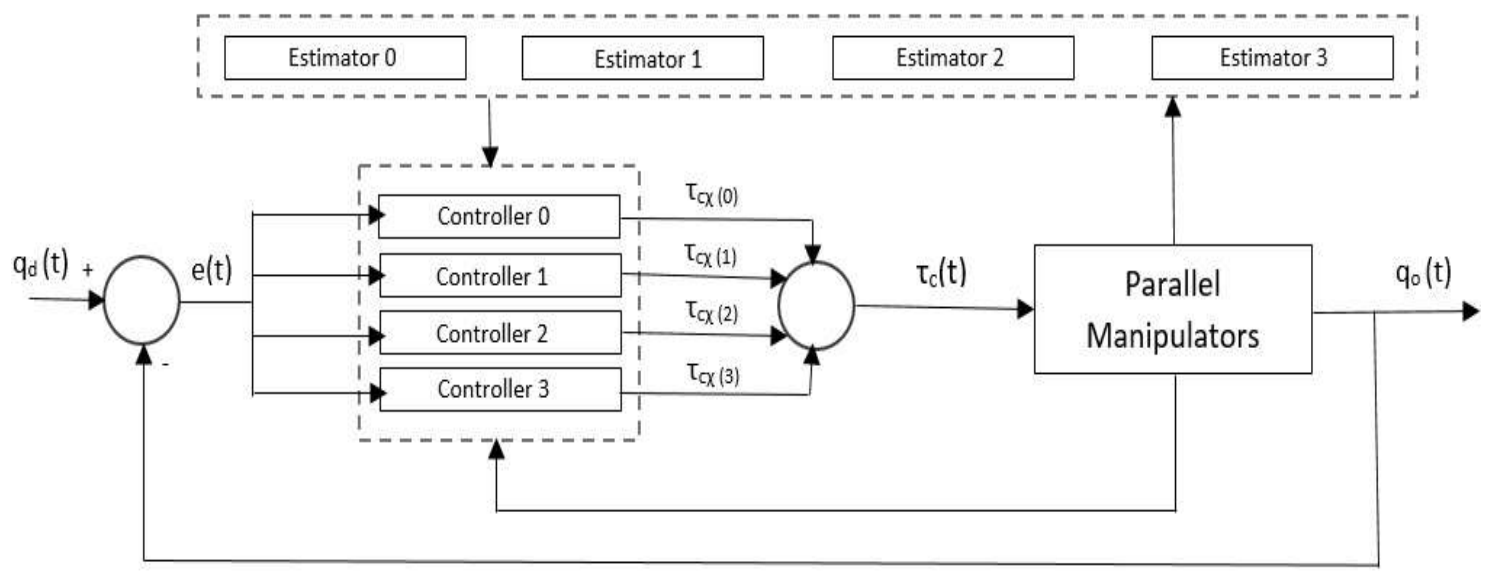

Figure 7.2: System Block Diagram.

patterns and the uncertain parameters in the system. In this section, we will explain the parameterization of the controller and develop adaptive laws for the estimators.

\subsubsection{Adaptive Controller Structure}

The adaptive control scheme is developed based on the nominal controller structure (7.27). Since we do not know the values of $\chi_{i}^{*}, \bar{\tau}$ and $\theta^{*}$, we first design the controller

$$
\tau_{c}(t)=\sum_{i=0}^{3} \tau_{c \chi(i)}(t)
$$

where $\tau_{c \chi(i)}(t)$ are the estimates of and $\chi_{i}^{*} \tau_{c(i)}^{*}$.

To derive $\tau_{c \chi(i)}(t)$, we first define $\tau_{c \chi(i)}(t)=\left[\alpha_{1} \tau_{c \chi(i) 1}, \alpha_{2} \tau_{c \chi(i) 2}, \alpha_{3} \tau_{c \chi(i) 3}\right]^{T}$, where $\alpha_{i}=0$ if actuator $i$ fails. Then, we calculate the elements of $\tau_{c \chi(i)}(t)$ from its subvector $\tau_{c \chi a(i)}$. The vector $\tau_{c \chi a(i)}$ is the same as $\tau_{c \chi(i)}(t)$ without the $i$ column. The vector $\tau_{c \chi a(i)}$ is developed from

$$
\chi_{i}^{*} \tau_{c a(i)}^{*}(t)=-\chi_{i}^{*} h_{i} K_{i 1} K_{d} s+h_{i} K_{i 1} Y \chi_{i}^{*} \theta^{*}+\chi_{i}^{*} \bar{\tau}_{i} h_{i} K_{i 2}
$$

From the actuator failure model, we can express unknown actuator failure signal as $\bar{\tau}_{i}(t)=\rho_{i}^{* T} f_{a i}(t)$, where $f_{a i}(t)=\left[1, f_{a i 1}(t), \ldots, f_{a i \bar{n}_{i}}(t)\right]^{T}$ are known functions cor- 
responding to the failure components, $\rho_{i}^{*}=\left[\bar{\tau}_{i 0}, \bar{\tau}_{i 1}, \ldots, \bar{\tau}_{i \bar{n}_{i}}\right]^{T}$ contains the parameter values associated with each actuator failure component and $\bar{n}_{i}$ is the number of failure components.

Finally, we can choose the controller $\tau_{c \chi a(i)}(t)$ as

$$
\begin{aligned}
\tau_{c \chi a(i)}(t)= & -\operatorname{diag}\left\{\chi_{i 1}, \chi_{i 2}\right\} h_{i} K_{i 1} K_{d} s(t) \\
& +\left[\begin{array}{c}
\eta_{i 1} \theta_{i 1} \\
\eta_{i n} \theta_{i 2}
\end{array}\right]+\left[\begin{array}{c}
\rho_{i(1)}^{T} f_{a i}(t) \phi_{i 1} \\
\rho_{i(2)}^{T} f_{a i}(t) \phi_{i 2}
\end{array}\right],
\end{aligned}
$$

where $\chi_{i j}$ is the estimate of $\chi_{i j}^{*}=\chi_{i}^{*}$ for $j=1,2, \theta_{i j}$ is the estimate of $\chi_{i j}^{*} \theta^{*}, \chi_{i j}^{*} \rho_{i}^{*}$ is estimated by $\rho_{i(j)}$ with $\eta_{i}=\left[\eta_{i 1}, \eta_{i 2}\right]^{T}=h_{i} K_{i 1} Y$ and $\phi_{i}=\left[\phi_{i 1}, \phi_{i 2}\right]^{T}=h_{i} K_{i 2}$. The function $f_{a i}(t)$ are known functions corresponding to component of the actuator which is defined in (8). From this, we can obtain $\tau_{c \chi(i)}(t)$ by adding the $i^{t} h$ zero column to $\tau_{c \chi a(i)}(t)$.

\subsubsection{Error System}

In order to design an adaptive law, we consider the estimation error from the estimates of actuator failures and unknown parameters. Our adaptive actuator failure compensation design ensures

$$
\begin{aligned}
J^{T} \tau(t) & =J^{T}(I-\sigma) \tau_{c}+J^{T} \sigma \bar{\tau} \\
& =J^{T}(I-\sigma)\left(\tau_{c}-\tau_{c}^{*}\right)+J^{T}(I-\sigma) \tau_{c}^{*}+J^{T} \sigma \bar{\tau} \\
& =J^{T}(I-\sigma)\left(\tau_{c}-\tau_{c}^{*}\right)+Y \theta^{*}-K_{d} s(t)
\end{aligned}
$$

as $J^{T}(I-\sigma) \tau_{c}^{*}+J^{T} \sigma \bar{\tau}=Y \theta^{*}-K_{d} s(t)$ ensured by the nominal design. 
The closed-loop system becomes

$$
E^{T} D_{c} E \dot{s}+E^{T}\left(D_{c} \dot{E}+C_{c} E\right) s+K_{d} s=J^{T}(I-\sigma(t))\left(\tau_{c}-\tau_{c}^{*}\right) .
$$

The error signal $\tilde{\tau}_{c}=\tau_{c}-\tau_{c}^{*}$ can be written as $\tilde{\tau}_{c}=\sum_{i=0}^{3} \tilde{\tau}_{c(i)}$,

where $\tilde{\tau}_{c(i)}=\left[\alpha_{1} \tilde{\tau}_{c a(i) 1}(t), \alpha_{2} \tilde{\tau}_{c a(i) 2}(t), \alpha_{3} \tilde{\tau}_{c a(i) 3}(t)\right]^{T}, \tilde{\chi}_{i}=\operatorname{diag}\left\{\tilde{\chi}_{i 1}, \tilde{\chi}_{i 2}\right\}, \tilde{\chi}_{i j}=\chi_{i j}-\chi_{i}^{*}$ and the error signal $\tilde{\tau}_{c a(i)}$, which represents the nonzero term of $\tilde{\tau}_{c a(i)}$ is

$$
\tilde{\tau}_{c a(i)}=\left[\begin{array}{c}
\tilde{\tau}_{c a(i) 1}(t) \\
\tilde{\tau}_{c a(i) 2}(t)
\end{array}\right]=-\tilde{\chi}_{i} h_{i} K_{i 1} K_{d} s(t)+\left[\begin{array}{c}
\eta_{i 1} \tilde{\theta}_{i 1} \\
\eta_{i n} \tilde{\theta}_{i 2}
\end{array}\right]+\left[\begin{array}{c}
\tilde{\rho}_{i(1)}^{T} f_{a i}(t) \phi_{i 1} \\
\tilde{\rho}_{i(n)}^{T} f_{a i}(t) \phi_{i 2}
\end{array}\right]
$$

with $\tilde{\rho}_{i(j)}=\rho_{i(j)}-\chi_{i}^{*} \rho_{i}^{*}$ for $j=1,2, \ldots, n$ and $\tilde{\theta}_{i j}=\theta_{i j}-\chi_{i j}^{*} \theta^{*}$.

\subsubsection{Adaptive Laws}

In this subsection, we develop an adaptive scheme for updating the estimated parameters of the controller (7.28). The adaptive laws for $\chi_{i j}$ with $i=0,1, \ldots, 3$ and $j=1,2$ are chosen as

$$
\dot{\chi}_{i j}=-\gamma_{i j} s^{T} \bar{J}_{(i) j} \mu_{i j}+P_{\chi_{i j}},
$$

where $\bar{J}_{(i) j} \in R^{n \times 1}$ represents the $j$ column of matrix $\bar{J}_{(i)}, \gamma_{i j}>0$ are the adaptation gains, $\mu_{i}=\left[\mu_{i 1}, \mu_{i 2}, \ldots, \mu_{i n}\right]^{T}=-h_{i} K_{i 1} K_{d} s(t), P_{\chi i j}$ is the projection function, $h_{i}$ and $K_{i 1}$ are the design matrices from the nominal controller structure.

The adaptive laws for $\rho_{i(j)}=\left[\rho_{i(j)(1)}, \ldots, \rho_{i(j)\left(\bar{n}_{i}\right)}\right]^{T}$ with $i=1, \ldots, 3$ and $j=1,2$ are chosen as

$$
\dot{\rho}_{i(j)}=-\Gamma_{(i j)} f_{a j} s^{T} \bar{J}_{(i) j} \phi_{i j}+P_{\rho_{i(j)}},
$$


where $P_{\rho i(j)}$ is the projection function and $\Gamma_{(i j)}=\Gamma_{(i j)}^{T}>0$ are the adaptation gain matrices.

The adaptive laws for $\theta_{i j}$ with $i=0,1, \ldots, m$ and $j=1,2, \ldots, n$ :

$$
\dot{\theta}_{i j}=-\beta_{i j} s^{T} \bar{J}_{(i) j} \eta_{i j}+P_{\theta_{i}}
$$

where $P_{\theta_{i}}$ is the projection function, $\eta_{i}=\left[\eta_{i 1}, \eta_{i 2}, \ldots, \eta_{i n}\right]^{T}=h_{i} K_{i 1} Y, \beta_{i j}=\beta_{i j}^{T}>0$ are the adaptation gain matrices.

Remark 7.2: Here we use a parameter projection design for $P_{\chi_{i j}}, P_{\rho_{i(j)}}$ and $P_{\theta_{i}}$. The parameter projection uses the knowledge of the parameter regions $0 \leq \chi_{i}^{*} \leq 1$, $\theta_{j}^{* a} \leq \theta_{i j}^{*} \leq \theta_{j}^{* b}$ and $\rho_{i k}^{* a} \leq \rho_{i k}^{*} \leq \rho_{i k}^{* b} . \rho_{i k}^{* a}$ and $\rho_{i k}^{* b}$ being the upper and lower bounds of the components $\rho_{i k}^{*}$ of $\rho_{i}^{*}$. $\theta_{j}^{* a}$ and $\theta_{j}^{* b}$ being the upper and lower bounds of each component in $\theta^{*}$.

This choice of $\varrho_{\chi}(t), \varrho_{\theta}(t)$ and $\varrho_{\rho}(t)$ ensures that $\left(\chi_{i j}-\chi_{i j}^{*}\right) P_{\chi_{i j}} \leq 0, \quad\left(\theta_{i}-\right.$ $\left.\chi_{i}^{*} \theta^{*}\right) P_{\theta}(t) \leq 0,\left(\rho_{i(j k)}-\chi_{i j}^{*} \rho_{i k}^{*}\right) P_{\rho_{i(j k)}} \leq 0$ which guarantees the boundedness of estimated parameters.

\subsubsection{Performance Analysis}

The proposed actuator failure compensation scheme can ensure the stability and tracking of the system with uncertain actuator failures as shown by the following theorem.

Theorem 7.1. For the system with unknown $D_{c}, C_{c}$ and $f$, the designed adaptive actuator failure compensation scheme guarantees the closed-loop signal boundedness and asymptotic output tracking: $\lim _{t \rightarrow \infty} e(t)=0$ despites the uncertainties of actua- 
tor failure index, failure time and failure values.

Proof: The Lyapunov function for no failure case is

$$
V_{0}=\frac{1}{2} s^{T} E^{T} D_{c} E s+\frac{1}{2} \sum_{k=0}^{2} \sum_{j=1}^{2} \tilde{\chi}_{k j}^{2} \gamma_{k j}^{-1}+\frac{1}{2} \sum_{k=0}^{2} \sum_{j=1}^{2} \tilde{\theta}_{k j}^{T} \beta_{k j}^{-1} \tilde{\theta}_{k j}+\frac{1}{2} \sum_{k=1}^{2} \sum_{j=1}^{2} \tilde{\rho}_{k(j)}^{T} \Gamma_{k j}^{-1} \tilde{\rho}_{k(j)} .
$$

The time derivative of the Lyapunov function $V_{0}$ is

$$
\dot{V}_{0} \leq-s^{T} K_{d} s \leq 0 .
$$

The Lyapunov function for actuator $i$ failure case is

$$
\begin{aligned}
& V_{i}=\frac{1}{2} s^{T} E^{T} D_{c} E s+\frac{1}{2} \sum_{k=0}^{2} \sum_{j=1}^{2} \tilde{\chi}_{k j}^{2} \gamma_{k j}^{-1}+\frac{1}{2} \sum_{k=0}^{2} \sum_{j=1}^{2} \tilde{\theta}_{k j}^{T} \beta_{k j}^{-1} \tilde{\theta}_{k j}+\frac{1}{2} \sum_{k=1}^{2} \sum_{j=1}^{2} \tilde{\rho}_{k(j)}^{T} \Gamma_{k j}^{-1} \tilde{\rho}_{k(j)} \\
& -\frac{1}{2} \sum_{k=0}^{2} \tilde{\chi}_{k i}^{2} \gamma_{k i}^{-1}-\frac{1}{2} \sum_{k=0}^{2} \tilde{\theta}_{k i}^{T} \beta_{k i}^{-1} \tilde{\theta}_{k i}-\frac{1}{2} \sum_{k=1}^{2} \tilde{\rho}_{k(i)}^{T} \Gamma_{k i}^{-1} \tilde{\rho}_{k(i)}
\end{aligned}
$$

The time derivative of the Lyapunov function $V_{i}$ is

$$
\dot{V}_{i} \leq-s^{T} K_{d} s \leq 0 .
$$

With the Lyapunov function, $s(t) \in L^{\infty} \bigcap L^{2}$ and all signals in the system are bounded, and so is $\dot{s}$; based on the Barbalat lemma, we can conclude that $\lim _{t \rightarrow \infty} s(t)=$ 0 and $\lim _{t \rightarrow \infty} e(t)=0$ 


\subsection{Simulations Study}

In order to demonstrate the effectiveness of the proposed control scheme, we implement an adaptive actuator failure compensation scheme for the 2-DOF redundantly actuated parallel manipulator. The parallel manipulator is actuated by three servo motors located at the bases A1, A2, and A3. The end-effector is mounted at the common joint $\mathrm{O}$, where the three manipulators meet. The joint angles are defined as follows: $q_{a 1}, q_{a 2}, q_{a 3}$ refer to the active joint angles, and $q_{b 1}, q_{b 2}, q_{b 3}$ refer to the passive joint angles.

\subsubsection{2-DOF redundantly actuated parallel manipulator model}

In this section, we discuss the dynamic of the parallel robotic system. We define $q_{o}=[x, y]^{T}$ as the position of the end-effector and $\tau_{a i}$ for $i=1,2,3$ is the force that each actuator produces. From (7.9), we have the dynamic of the system as [16]

$$
E^{T} D_{c} E \ddot{q}_{o}+E^{T}\left(D_{c} \dot{E}+C_{c} E\right) \dot{q}_{o}+J^{T} f_{a}=J^{T} \tau_{a} .
$$


The matrices $D_{c}$ and $C_{c}$ are

$$
D_{c}=\left[\begin{array}{cccccc}
\theta_{1}^{*} & 0 & 0 & \theta_{7}^{*} c_{a b 1} & 0 & 0 \\
0 & \theta_{2}^{*} & 0 & 0 & \theta_{8}^{*} c_{a b 2} & 0 \\
0 & 0 & \theta_{3}^{*} & 0 & 0 & \theta_{9}^{*} c_{a b 3} \\
\theta_{7}^{*} c_{a b 1} & 0 & 0 & \theta_{4}^{*} & 0 & 0 \\
0 & \theta_{8}^{*} c_{a b 2} & 0 & 0 & \theta_{5}^{*} & 0 \\
0 & 0 & \theta_{9}^{*} c_{a b 3} & 0 & 0 & \theta_{6}^{*}
\end{array}\right]
$$

with $\theta_{i}^{*}$ represents the system parameters which are unknown to the controller, the functions $c_{a b i}=\cos \left(q_{a i}-q_{b i}\right), s_{q b i}=\left(\sin q_{a i}-q_{b i}\right) \dot{q}_{b i}$, and $s_{q a i}=-\left(\sin q_{a i}-q_{b i}\right) \dot{q}_{a i}$. 
The known Jacobian matrices $E$ and $J$ are

$$
E=\left[\begin{array}{cc}
r_{1} \cos q_{(b 1)} & r_{1} \sin q_{(b 1)} \\
r_{2} \cos q_{(b 2)} & r_{2} \sin q_{(b 2)} \\
r_{3} \cos q_{(b 3)} & r_{3} \sin q_{(b 3)} \\
-r_{1} \cos q_{(a 1)} & -r_{1} \sin q_{(a 1)} \\
-r_{2} \cos q_{(a 2)} & -r_{2} \sin q_{(a 2)} \\
-r_{3} \cos q_{(a 3)} & -r_{3} \sin q_{(a 3)}
\end{array}\right], J=\left[\begin{array}{cc}
r_{1} \cos q_{(b 1)} & r_{1} \sin q_{(b 1)} \\
r_{2} \cos q_{(b 2)} & r_{2} \sin q_{(b 2)} \\
r_{3} \cos q_{(b 3)} & r_{3} \sin q_{(b 3)}
\end{array}\right],
$$

where $r_{i}=\frac{1}{l \sin \left(q_{b i}-q_{a i}\right)}$ and $l$ represents the length of each link, which can be obtained easily.

\subsubsection{Simulation Conditions}

In the simulation study, we assign the parameters in the system as $\theta_{1}^{*}=0.0932, \theta_{2}^{*}=$ $0.0427, \theta_{3}^{*}=0.0427, \theta_{4}^{*}=0.0381, \theta_{5}^{*}=0.0085, \theta_{6}^{*}=0.0085, \theta_{7}^{*}=0.0426, \theta_{8}^{*}=$ $0.0111, \theta_{9}^{*}=0.0111$. The friction parameters are chosen as $f_{v 1}=2.9936, f_{v 2}=$ $2.7617, f_{v 3}=2.8771, f_{c 1}=0.4976, f_{c 2}=0.4570, f_{c 3}=0.3006$. These parameters are only used for the simulation and are unknown to the controller.

The initial conditions of the system are chosen as $q_{o}=\left[\begin{array}{ll}0.3 & 0.3\end{array}\right]^{T}$, the reference signal is chosen as $q_{d}(t)=[2.22 .9]^{T}$.

\subsubsection{Simulation Results}

In this section, we show some of our preliminary results of our study. In this case, we only consider simple cases of simulation with constant reference input and constant actuator failure. To simplify the problem, we assume that most of the parameters in the system are known with only the exception of $\theta_{1}^{*}$ and $\theta_{2}^{*}$. 
In this simulation, we use constant actuator failure signals. We let actuator $q_{a 1}$ failure occurs twice as follows:

(i) No actuator failure case: $\tau(t)_{a}=\tau_{c}(t)$ for $0 \leq t<50 \mathrm{~s}$,

(ii) Actuator $q_{1}$ failure case: $\tau_{a 1}=\bar{\tau}_{1}=5$ at $50 \leq t<100 \mathrm{~s}$,

(iii) The failing actuator $q_{1}$ becomes normal again, no actuator failure case: $\tau(t)=$ $\tau_{c}(t)$ for $100 \leq t<150 s$,

(iv) Actuator $q_{1}$ failure case: $\tau_{a 1}=\bar{\tau}_{1}=-5$ at $150 \leq t<200 s$.

In this case, the simulation results show the output of the system in Figure 7.3, the position of the actuated joint $q_{a 1}, q_{a 2}, q_{a 3}$ in Figure 7.4 and the estimated failure torque $\bar{\tau}_{1}$ are presented in Figure 7.5 .
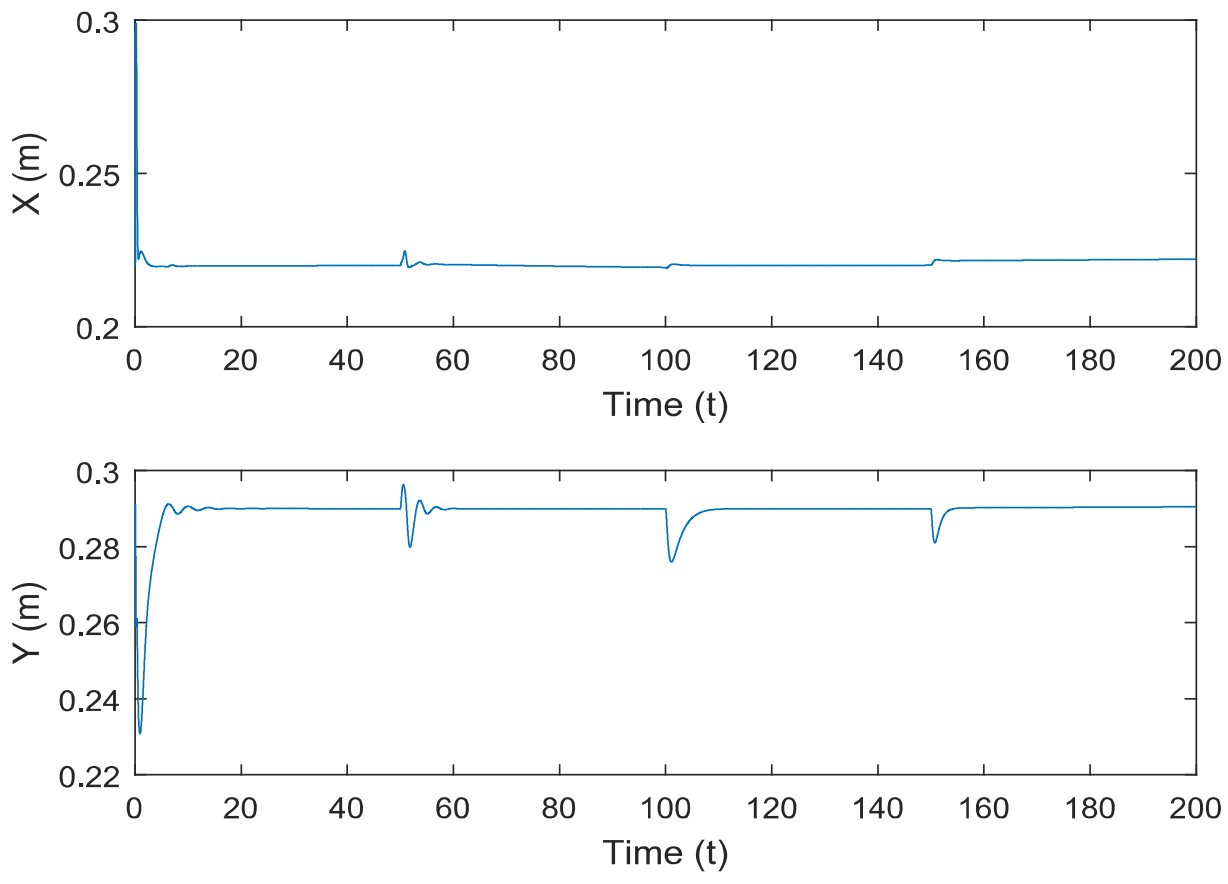

Figure 7.3: System output $q_{o}(t)$ for a reference $q_{d}=[2.22 .9]^{T}$. 

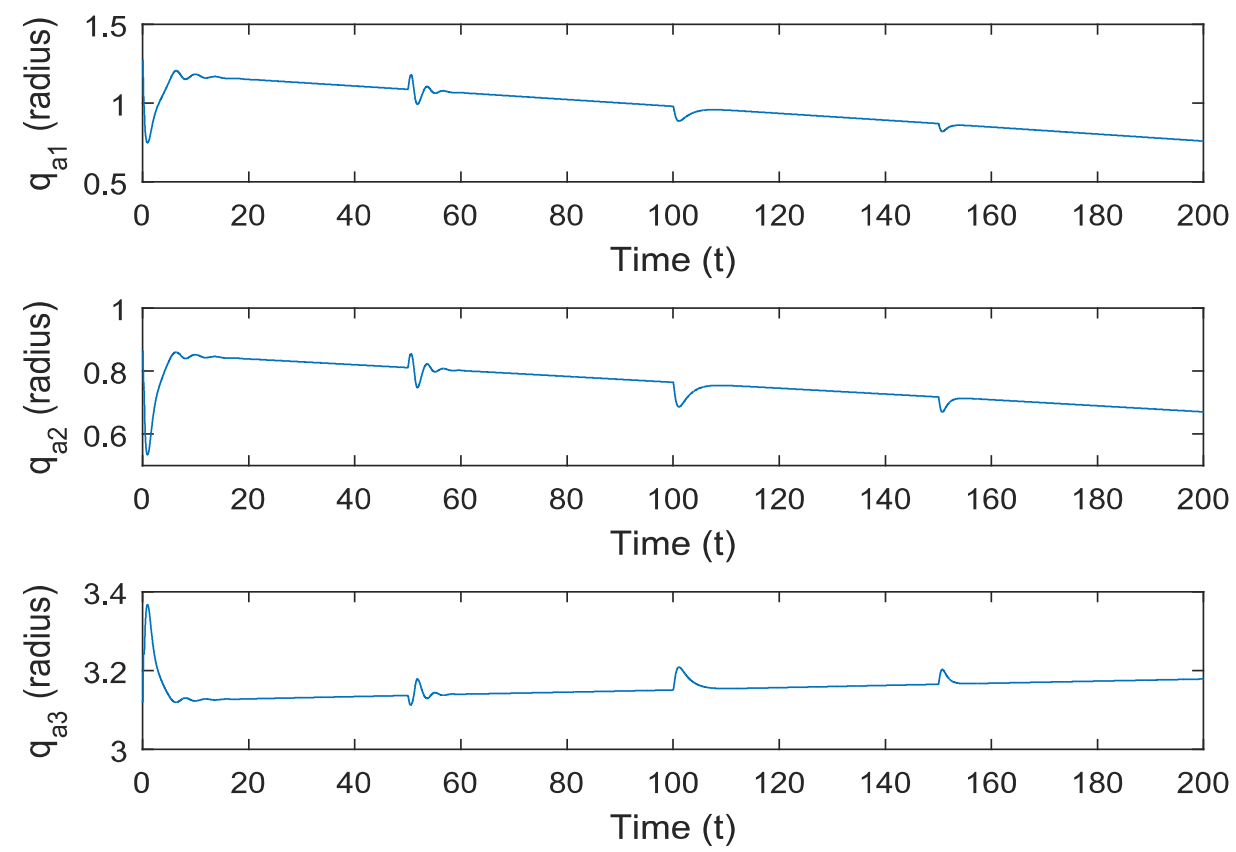

Figure 7.4: Angle of the actuated joint $q_{a 1}, q_{a 2}, q_{a 3}$.
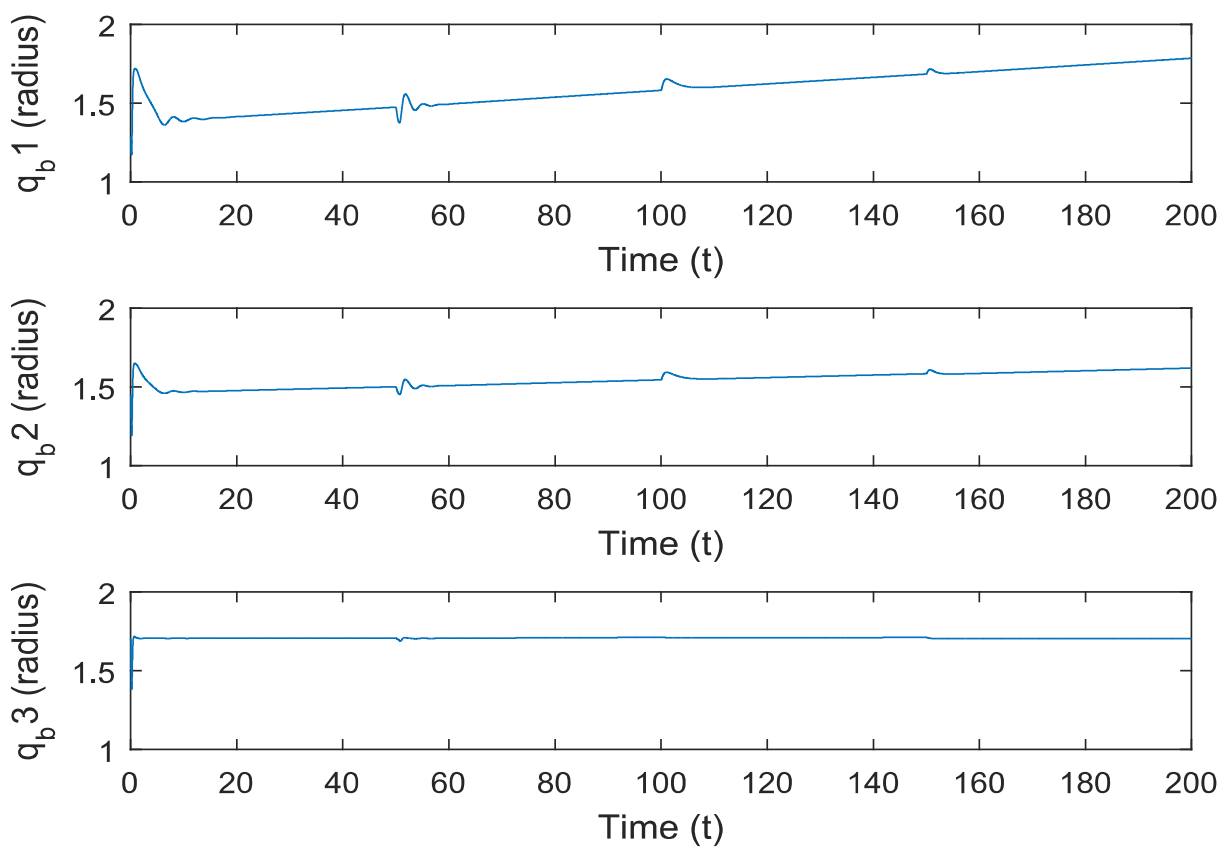

Figure 7.5: Angle of the flexible joint $q_{b 1}, q_{b 2}, q_{b 3}$. 

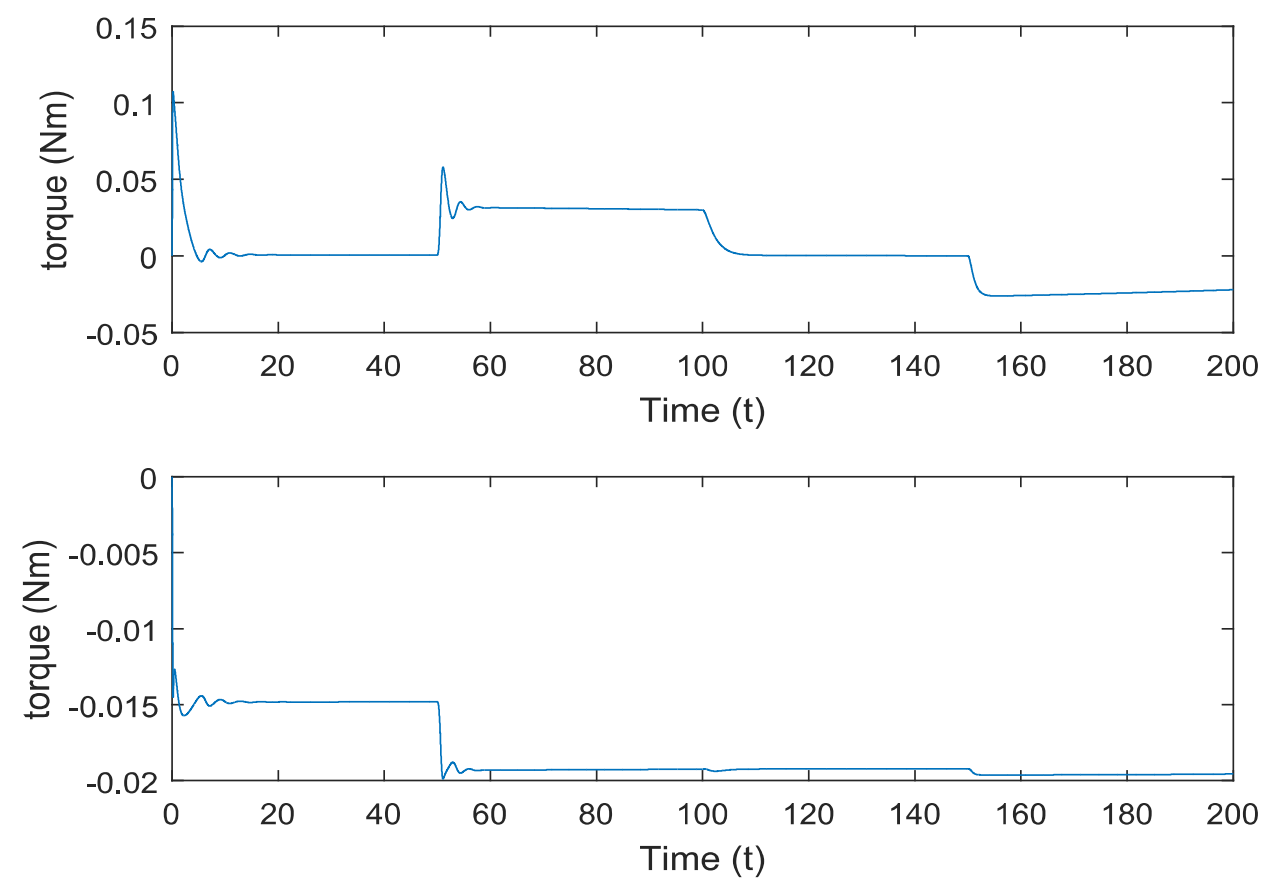

Figure 7.6: Estimated value of the failure torque $\bar{\tau}_{1}$.

The simulation results confirm that the adaptive actuator failure compensation scheme can guarantee that the tracking error of the system goes to zero for the desired trajectory.

\subsection{Conclusions}

A parallel manipulator robotic system plays an important role in many projects and the concept of adding redundancy to the system can be used to improve the performance as well as reliability of a robotic system. Developing an adaptive actuator failure compensation scheme for parallel robots that can immediately compensate for any possible failures in the system is important for the control and robotic fields.

In this chapter, we have studied the adaptive actuator failure compensation scheme with a parallel manipulator robotic system. Our research shows that, with a complete parameterization of failure patterns and system parameters, a desirable closed-loop 
stability and asymptotic tracking of the system can be achieved, despite uncertain actuator failures. The simulation results also verified the performance of the adaptive control algorithm when applied to the 2-DOF redundantly actuated parallel manipulator model subject to uncertain actuator failures. 


\section{Chapter 8}

\section{Design for Landing of A Helicopter with Robotic Legs}

A helicopter is a type of aircraft, which is capable of taking off and landing vertically. This attribute allows helicopters to be used in congested or isolated areas and perform tasks that is not possible for other types of aircraft. One limitation of the current state-of-the-art helicopter is that the system needs to land on a flat surface. In order to overcome this limitation, engineers proposed a new type of robotic landing gear using robot manipulators, which fold in flight, extract while landing, and bend in response to variable terrains [14]. These robotic legs allow for stable and safe landing of unmanned helicopters in a variety of rough terrains which are typically not accessible to traditionally outfitted aircrafts. The proposed system is still in the developmental state and there are several issues that are needed to be addressed before the proposed landing system can be used in a practical situation.

Safety is one of the most important problems that needs to be solved before the proposed landing system can be used. Although the robotic landing gear can help a helicopter operates on an uneven terrain, this approach also increases the complexity of the landing process. The robotic landing gear using legs with articulated joints that can fold up when the helicopter is flying and extend when the copter is preparing to land, thus the system needs to deal with several actuators in each manipulator. 
In order to address the aforementioned problems, a more advanced control design is needed to control the landing process of the helicopter with robotic legs subject to uncertain actuator failures.

Additionally, the proposed helicopter landing system needs to operate on several types of uneven terrains and in various circumstances, which many parameters of the system and the environment may be unknown. To deal with the uncertainties, the problem of designing adaptive control laws for rigid-robot manipulators that ensures asymptotic trajectory tracking is needed. The basic idea of adaptive control is to change the values of gains or parameters in the control law according to some online algorithm corresponding to the changes and uncertainties in the system. The adaptive controller can learn an appropriate set of controller parameters to compensate actuator failures through the adaptation for each successive operation $[15,16,17,18,19]$.

The studied helicopter landing system utilizes the parallel structure in the manipulator legs to make adjustments in the landing process. A parallel manipulator is a mechanical system that uses several computer-controlled serial chains to support a single platform, in this case the body of a helicopter. With a parallel manipulator structure, a high rigidity may be obtained with a small mass of the manipulator, which allows high precision with high speed of movements, and the redundancy of the actuators can potentially be used to solve the actuator failure problem [20, 21]. Over the past two decades, parallel manipulators have attracted the attention of many researchers. However, most literature focuses on the studies of kinematics and dynamics of the system $[22,23]$. Researches on control strategies are relatively few especially regarding to actuator failure compensation problem. In the literature, there are two types of basic control strategies for parallel manipulators: kinematic control strategies and dynamic control strategies. In the kinematic control strategies, the nonlinear dynamics are not considered to avoid the complex computation, thus, the 
controller can be designed easily. However, the landing process of a helicopter is a complicated and rapid process, these types of controllers can not always produce satisfactory performance, and there is no guarantee of stability especially at higher speed [24]. On the other hand, the dynamic control strategies, which are considered in this study use a full dynamic model of the system. In this way, the nonlinear dynamics of the landing mechanism can be compensated and higher performance can be achieved $[25,26,27]$. Finally, this control approach allows for actuator failure compensation of the landing mechanism, which is the main focus of this study.

Actuator failure compensation is a well-known problem in control system. There are studies that focus on actuator failure compensation, such as the neural network control, sliding-mode control, and an adaptive actuator failure compensation [28, 29]. However, most studies only focus on actuator degradation which only consider small changes in the actuator outputs, or rely on the detection and isolation scheme of actuator failures in the system which is relatively slow and its used is limited $[30,31$, $32,33]$. In order to efficiently compensate actuator failures in the helicopter landing process, an actuator failure compensation control scheme that is reliable and capable of immediate failure adaptation is needed to ensure a safe landing [34, 35].

In this paper, we develop a new adaptive actuator failure compensation scheme for a helicopter landing system with parameter uncertainties. The algorithm uses redundancy in the parallel structure to ensure desired closed-loop stability and asymptotic output tracking of the system subject to uncertain actuator failure. This paper makes the following contributions to solving such a new problem:

- Develop an adaptive actuator failure compensation scheme for landing of a Helicopter with parameter uncertainties, in addition to actuator failures.

- Study the dynamic and control for the landing of a helicopter using robotic legs, and demonstrate how to deal with the constraint of the parallel structure. 
- Clerify several key technical issues and solutions for the adaptive actuator failure compensation scheme for helicopter landing system and extend the failure compensation scheme to concurrent actuator failure case.

- Study the simulation of the adaptive actuator failure compensation scheme based on a Helicopter landing system subject to uncertain actuator failure.

The paper is organized as follows. In Section II, we formulate the problem with the discussion of the dynamic model of a proposed helicopter landing system, actuator redundancy, actuator failure model and control objective. In Section III, we design a nominal controller by assuming that the knowledge of actuator failures is known. In Section IV, we develop the adaptive control scheme based on the nominal controller structure. Finally, in Section V, we study the simulation of a helicopter landing system to confirm the effectiveness of the control design.

\subsection{Problem Formulation}

The objective of this research is to develop an adaptive actuator failure compensation scheme to solve the actuator failure problem in the proposed helicopter landing mechanism. In this study, we utilize the redundancy in the structure of a parallel manipulator to ensure closed-loop stability and asymptotic output tracking of the system with parameter uncertainties in the presence of uncertain actuator failures.

\subsubsection{Dynamic Model of the System}

In this study, we will develop an adaptive actuator failure compensation scheme for a helicopter landing system on uneven terrains with redundantly actuated parallel manipulator as. 


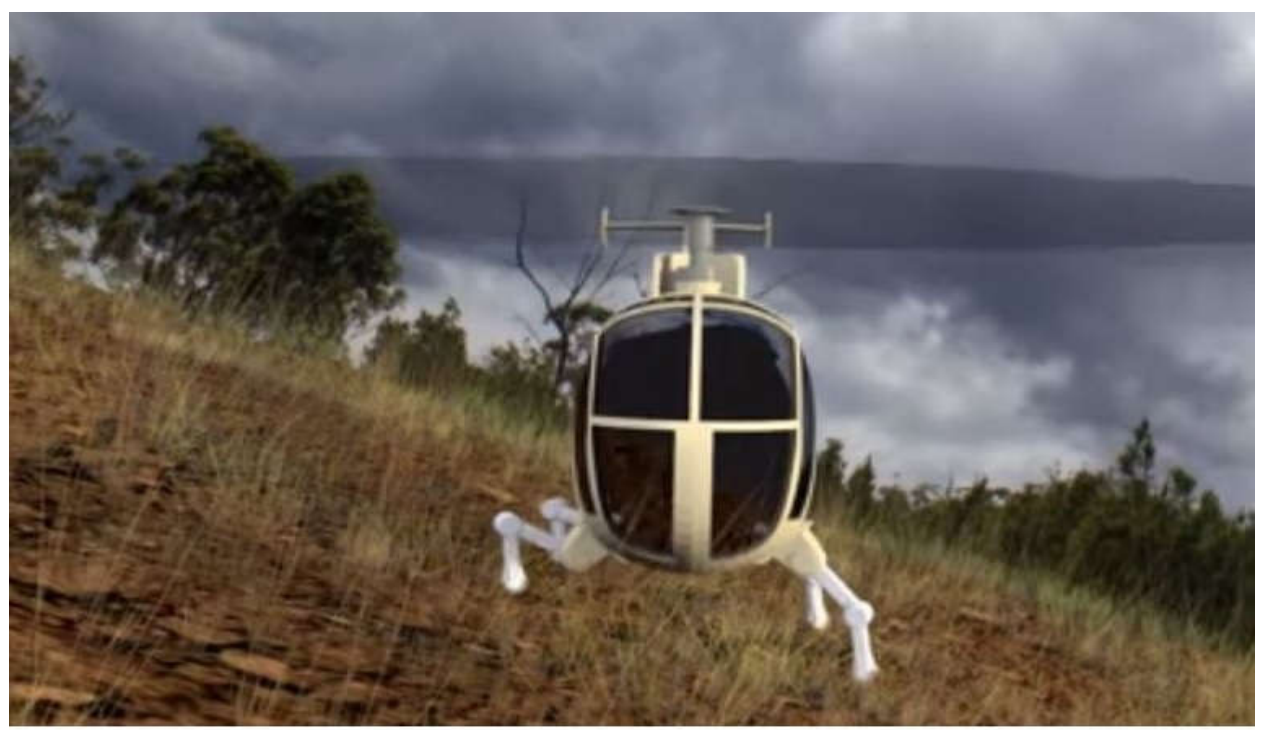

Figure 8.1: Landing of a helicopter with robotic legs on uneven terrains with redundantly actuated parallel manipulators.

In order to improve the performance of the landing system and guarantee a safe landing, we propose a new structure of the helicopter landing system by using six landing legs instead of four legs that is originally proposed. The Hexapod structure of the landing mechanism could improve the system performance and the additional redundancy could be used to design an effective adaptive actuator failure compensation controller for the system.

The first step in designing the controller for the system is to study the dynamic model of the new proposed landing mechanism. The dynamic model of the system can be obtained by combining the dynamic model of each landing leg with the dynamic model of the helicopter body.

Dynamic model of each landing leg: In this study, we use a two-link robotic manipulator as each leg in the landing mechanism of the helicopter [26]. The dynamic model of such leg can be written as

$$
D_{i} \ddot{q}_{i}+C_{i} \dot{q}_{i}+g_{i}=\tau_{i}-J_{a i}^{T} f_{e x t(i)},
$$


where $\ddot{q}_{i}$ and $\dot{q}_{i}$ are the joint angular acceleration and angular velocity of each manipulator for $i=1,2, \ldots, 6, D_{i} \in R^{2 \times 2}$ is inertia matrix, and $C_{i} \in R^{2 \times 2}$ is Coriolis and centrifugal term, $g_{i}$ is the gravitational term $J_{a i} \in R^{2 \times 2}$ is the nonsingular Jacobian matrix and $f_{\text {ext(i) }}$ is the external force vector at the end-effector.

Dynamic model of a helicopter body: Because the main focus of this research is the landing mechanism of the helicopter, we model the body of the helicopter as a rigid mass with time varying parameters for its mass and inertia. The dynamic model of the helicopter can be written as

$$
\begin{gathered}
I_{h} \ddot{q}_{h}+C_{h}\left(q, \dot{q}_{h}\right) \dot{q}_{h}=\tau_{h} \\
M_{h} I_{3} \ddot{p}=f_{h},
\end{gathered}
$$

where $p_{o} \in R^{3}$ is the augular displacement of the helicopter body, $p \in R^{3}$ is the position of the payload, $I_{h}$ is a $3 \times 3$ matrix of the inertia, $C_{p}(q, \dot{q})$ represents Coriolis and centrifugal term, $M_{h}$ is the effective mass of the helicopter that the landing mechanism need to support, $I_{3}$ is a $3 \times 3$ identity matrix, and $\tau_{h} \in R^{3}$ and $f_{h} \in R^{3}$ are the generalized torque and force exerted on the helicopter body.

From the general dynamic model of a rigid body, we can see that the system has six degrees of freedom. However, for the landing aspect of a helicopter, the landing mechanism is set up directly under the base of the helicopter such that the upward force of each manipulator will only affect the height displacement, roll angle and pitch angle of the helicopter body, which are enough to guarantee a safe landing. From this, the dynamic model of the helicopter body is reduced to

$$
D_{o} \ddot{q}_{o}+C_{o} \dot{q}_{o}=\tau_{o}
$$


where $q_{o} \in R^{3}$ is the height, roll and pitch of the helicopter body, $D_{o}$ is a $3 \times 3$ inertia matrix, $C_{o}$ represent Coriolis and centrifugal terms, and $\tau_{o} \in R^{3}$ are the generalized torques exerted on the body that is related to height, roll and pitch of the helicopter.

System constraint: The combined dynamic of the manipulator with system constraint can be written as $[27]$

$$
D_{m} \ddot{q}+C_{m} \dot{q}+g=\tau-J_{a}^{T} f_{e x t}+A^{T} \lambda,
$$

where $D_{m} \in R^{12 \times 12}$ is the combined inertia matrix of the manipulator, $C_{m} \in R^{12 \times 12}$ is the combined Coriolis and centrifugal matrix, $\tau$ is the torque vector, $f_{e x t}$ is a force that is exerted on the helicopter body and $q$ represents the angle of each manipulator joint in the system.

One of the difficult problems, which arises in the robotic system with parallel structure, is how to deal with the constraints. The constrained torques in the system are difficult to measure; as a result, we cannot directly develop a controller that addresses this issue. In this study, we first consider the constraint force vector $A^{T} \lambda$, where the matrix $A$ is the differential of the closed-loop constrained equation $\frac{\partial H(q)}{\partial t}=$ $A(q) \dot{q}=0$ and $\lambda$ is a multiplier representing the magnitude of the constraint force.

In order to deal with the unknown constraint force vector $A^{T} \lambda$, we define the Jacobian matrix $E$ such that $\dot{q}=E \dot{q}_{o}$ with $\dot{q}_{o}$ represents the vertical velocity, pitch rate and roll rate of the helicopter. With the Jacobian matrix $E$ and the fact that $A(q) \dot{q}=0$, we have $A E \dot{q}_{o}=0$, from which one can conclude that the equation $E^{T} A^{T}=0$ holds. With this property and the relation $\ddot{q}=\dot{E} \dot{q}_{o}+E \ddot{q}_{o}$, the dynamic 
model of the parallel manipulator with constraints can be written as

$$
\begin{aligned}
E^{T} D_{m} E \ddot{q}_{o} & +E^{T}\left(D_{m} \dot{E}+C_{m} E\right) \dot{q}_{o}+E^{T} g \\
& =E^{T} \tau-E^{T} J_{a}^{T} f_{\text {ext }} .
\end{aligned}
$$

This dynamic model represents the dynamic of the leg structure with constraint. In the next subsection, we consider the combined dynamic of the helicopter body with the manipulator dynamic.

Combind dynamic model of the system: The combined dynamic of the system can be obtain with the substitution of the generalized torque $\tau_{o}$ with the generalized external force of each manipulator $f_{\text {ext }}$. Defind a transformation matrix $J_{b} \in R^{3 \times 3}$ such that $J_{b} \tau_{o}=E^{T} J_{a}^{T} f_{\text {ext }}$. The dynamic model of the system can be expressed as

$$
\begin{gathered}
E^{T} D_{m} E \ddot{q}_{o}+E^{T}\left(D_{m} \dot{E}+C_{m} E\right) \dot{q}_{o}+E^{T} g \\
+J_{b} D_{o} \ddot{q}_{o}+J_{b} C_{o} \dot{q}_{o}=E^{T} \tau .
\end{gathered}
$$

Because the proposed landing mechanism needs to operate on various circumstances, many parameters in the landing process may be unknown. This means that there are some uncertainties in the $D_{m}, C_{m}, g, D_{o}, C_{o}$ matrices. In this study, we only focus on the control of the landing mechanism not the control of the flight mechanic in the helicopter, thus, any time varying function related to the flight mechanic of the helicopter is assumed to be known.

In a robotic system, it is common to assume that the system satisfies the following structural properties:

a) $D_{m}, D_{o}$ is a symmetric and positive definite matrix.

b) $\dot{D}_{c}-2 C_{c}, \dot{D}_{o}-2 C_{o}$ is a skew symmetric matrix. 
With this assumption, it can be proven that the new dynamic model that we obtained (8.6) also satisfies the similar structural properties:

c) The combined inertia matrix $E^{T} D_{m} E+J_{b} D_{o}$ is a symmetric and positive definite matrix.

d) The combined matrix $\frac{\partial E^{T} D_{m} E+J_{b} D_{o}}{\partial t}-2\left(E^{T}\left(D_{m} \dot{E}+C_{m} E\right)+J_{b} C_{o}\right)$ is a skew symmetric matrix.

Remark 8.1: The helicopter may land on various terrains, the Jacobian matrix $E$ may change with each landing. However, we can easily measure the structure of the landing surface from the position of each landing leg on the initial impact when the helicopter landed. As a result, the Jacobian matrix is assumed to be known for each landing.

\subsubsection{Actuator Redundancy}

In order to develop an actuator failure compensation scheme, having redundancy in the system is one of the most crucial requirements. The proposed helicopter landing system with six manipulators satisfies this requirement; thus, the study of actuator failure compensation scheme for the systems could improve both the performance and reliability of the proposed landing mechanism.

The adaptive actuator failure compensation scheme developed in this study utilizes the redundancy from the parallel structure of the landing mechanism to compensate for possible actuator failures. In order to achieve this objective, the helicopter landing system considered in this study requires at least 3 functioning actuators in different manipulator, thus, we allows actuator failures for up to 3 different manipulators in each actuator failure case.

In this study, we define actuator failures in the system as follows: Let $\Sigma$ be a 
set of all possible actuator failure pattern such that $\Sigma=\left\{\sigma_{(1)}, \sigma_{(2)}, \ldots, \sigma_{(N)}\right\}$, where $N$ is the number of all possible actuator failure pattern that satisfies Assumption 1, and $\sigma_{(i)}=\operatorname{diag}\left\{\sigma_{(i) 1}, \sigma_{(i) 2}, \ldots, \sigma_{(i) 12}\right\}$ is the actuator failure pattern with $\sigma_{(i) j}=1$ if actuator $j$ fails and $\sigma_{(i) j}=1$ otherwise.

Assumption 1: Define a matrix $\bar{E}_{(i)}$, for each actuator failure pattern $\sigma_{(i)}$, as a submatrix of $E$ without the columns associated with the failing actuator (each associated column $j$ is such that $\sigma_{(i) j}=1$ ). In this study, we assume that the rank of the matrix $\bar{E}_{(i)}$ must be larger or equal to 3 for every possible actuator failure pattern.

Remark 8.2: Although the proposed landing mechanism contain twelve actuators, the actuator located on the same manipulator is dependent with each other. This means that an actuator failure in a manipulator will severely affect the performance of the entire manipulator, thus, in most case it is not possible to use the other actuator within the same manipulator to compensate for each other, especially when the manipulator needs to support a large load such as in the helicopter landing system considered in this study.

\subsubsection{Actuator Failure Model}

Our adaptive control scheme needs to be able to compensate for an actuator failure in the helicopter landing system, but it is a difficult task to obtain the exact knowledge of the actuator failures that have yet to occur. To design an adaptive actuator failure compensation scheme, we first develop an actuator failure model to describe the unknown actuator failures in the system as follows.

Let $\tau(t)=\left[\tau_{1}, \tau_{2}, \ldots, \tau_{12}\right]^{T}$ be the system input vector. When an actuator failure occurs, the control input component $\tau_{j}(t)$ associated with the failing actuator $j$ may 
become an arbitrary signal, which can be expressed as [34]

$$
\tau_{j}(t)=\bar{\tau}_{j}(t), t \geq t_{j} .
$$

Although the exact value of the control input $\bar{\tau}_{j}(t)$ is unknown, the structure of actuator failure can be obtained based on some knowledge of the actuator as

$$
\bar{\tau}_{j}(t)=\bar{\tau}_{j 0}+\sum_{k=1}^{\bar{\rho}} \bar{\tau}_{j k} f_{a j k}(t), t \geq t_{j},
$$

where $\bar{\rho}$ is the number of components in the failing signal, $f_{a j k}$ are known functions corresponding to each component of the actuator and $j, t_{j}, \bar{\tau}_{j k}$ are unknown failure index, failure time and failure value of each actuator component.

With some specifications of the unknown constants $\bar{\tau}_{j 0}, \bar{\tau}_{j k}$ and known functions $f_{a j k}(t)$, we can model most common actuator failures such as the lock in-place failure of the actuator: $\bar{\tau}_{j}=\bar{\tau}_{j 0}$, which may happen when the controller fails to communicate with the actuator, or the complete failure of a motor in the system: $\bar{\tau}_{j}=0$. Addition-

ally, the terms $\bar{\tau}_{j k} f_{a j k}(t)$ in the actuator failure model can be used to model additional types of time varying actuator failures such as a square wave actuator failure, which the failure occurs periodically.

\subsubsection{Control Objective}

Our control objective is to guarantee the height displacement, roll angle and pitch angle of the helicopter body, or $q_{o}(t)$ tracks a desirable trajectory $q_{d}(t)$ asymptotically even when an actuator failure occurs. Consider the combined system model (8.6), due to possible actuator failures, an applied feedback control signal $\tau_{c}(t)$ may not reach the system, as $\tau_{j}(t)$ is not equal to $\tau_{c j}(t)$. More precisely, for each actuator failure 
pattern $\sigma_{(i)}$, the signal $\tau(t)$ is

$$
\tau(t)=\left(I-\sigma_{(i)}(t)\right) \tau_{c}(t)+\sigma_{(i)}(t) \bar{\tau}_{(i)}
$$

where $\bar{\tau}_{(i)}$ is an unknown failure vector for the actuator failure pattern $\sigma_{(i)}$ case, and $\sigma_{(i)}(t)$ is the actuator failure pattern matrix such that $\sigma_{(i) j}(t)=1$ if the $j$ actuator fails and $\sigma_{(i) j}(t)=0$ otherwise.

The control objective is to design a feedback control signal $\tau_{c}(t)$ such that the closed-loop system satisfies: $E^{T} D_{m} E \dot{s}+E^{T}\left(D_{m} \dot{E}+C_{m} E\right) s+J_{b} D_{o} \dot{s}+J_{b} C_{o} s+K_{d} s=0$ in the nominal case, and also asymptotically in the adaptive case of unknown system parameters, despite the uncertain actuator failures $\tau(t)=\left(I-\sigma_{(i)}(t)\right) \tau_{c}(t)+\sigma_{(i)}(t) \bar{\tau}$, where the error vector $s$ is defined as $s=\dot{e}+\Lambda_{0} e$ with $e=q_{o}-q_{d}, q_{d}$ is the desired trajectory of the end-effector, $\Lambda_{0} \in R^{2 \times 2}$ is a design matrix whose eigenvalues have positive real parts and the gain matrix $K_{d}$ is chosen to be positive definite. When this objective is met, the system will be ensured to have the desired signal boundedness and tracking properties $\lim _{t \rightarrow \infty} e(t)=0$.

\subsection{Nominal control design}

The adaptive actuator failure compensation scheme proposed in this study is a control scheme that builds on top of the existing control algorithm. The proposed scheme enable the existing algorithm to be able to compensate for any possible actuator failure.

From the block diagram, each nominal controller in the controller bank is developed based on an existing control algorithm that is designed for the system without actuator failure such as the backstepping control or the Slotine and Li's control design. In this case, the actuator failure values are known, and the switching scheme is 


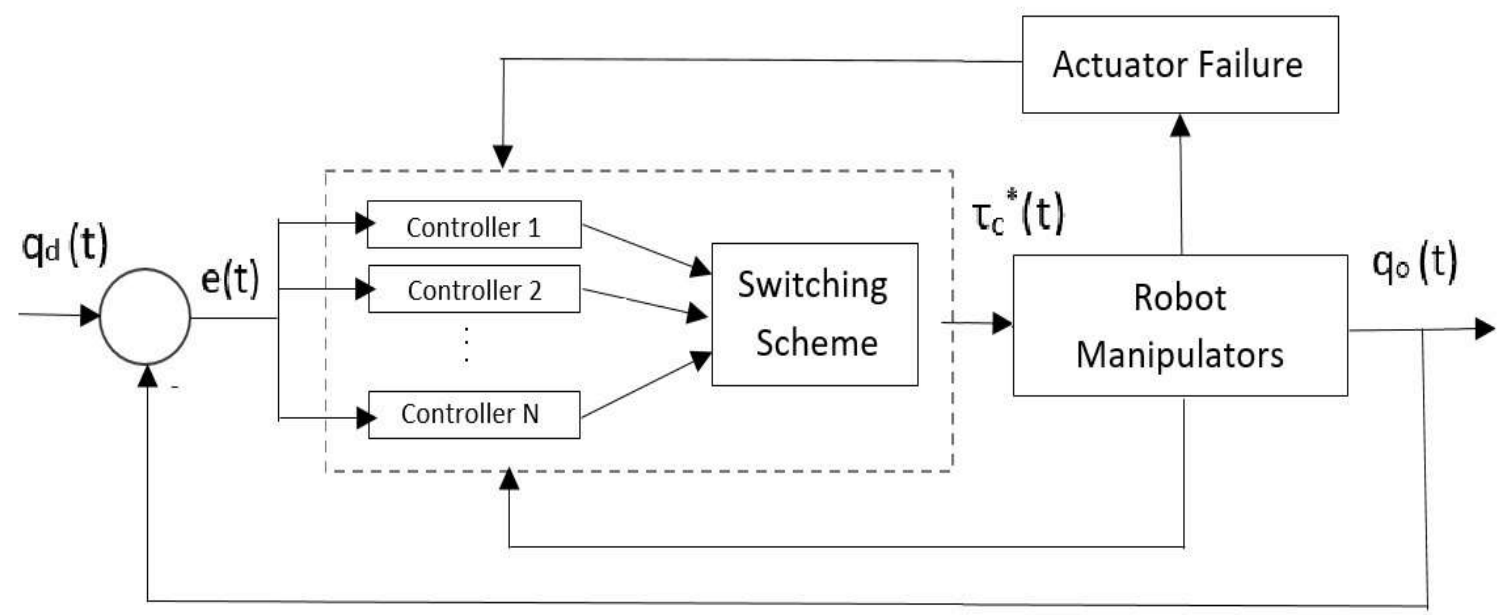

Figure 8.2: Block diagram for nominal control design

developed from the known actuator failure pattern.

In this section, we develop a nominal controller for the proposed helicopter landing system by assuming that the knowledge of the actuator failures is known. Such a controller structure is needed to ensure that it is possible to design the adaptive actuator failure. Moreover, the baseline control design developed in this section can be used to design an adaptive actuator failure compensation scheme with uncertain actuator failures in the following section.

\subsubsection{Ideal Control System}

Before we begin the development of the actuator failure compensation, we first study the conventional controller for the system, which is developed based on the Slotine and Li's approach. Consider the dynamic model of the helicopter landing system (8.6) as

$$
\begin{gathered}
E^{T} D_{m} E \ddot{q}_{o}+E^{T}\left(D_{m} \dot{E}+C_{m} E\right) \dot{q}_{o}+E^{T} g \\
+J_{b} D_{o} \ddot{q}_{o}+J_{b} C_{o} \dot{q}_{o}=E^{T} \tau .
\end{gathered}
$$


In order to develop a controller for the system, we define an error vector $s=$ $\dot{e}+\Lambda_{0} e \in R^{3}$, where $e=q_{o}-q_{d}, v=\dot{q}_{d}-\Lambda_{0} e \in R^{3}$ being an intermediate vector signal and let $\Lambda_{0}$ be a constant matrix whose eigenvalues have positive real parts. We can parameterize the system with

$$
\begin{gathered}
E^{T} D_{m} E \ddot{q}_{o}+E^{T}\left(D_{m} \dot{E}+C_{m} E\right) \dot{q}_{o}+E^{T} g+J_{b} D_{o} \ddot{q}_{o} \\
+J_{b} C_{o} \dot{q}_{o}=Y\left(q_{o}, \dot{q}_{o}, v, \dot{v}\right) \theta^{*}+F\left(q_{o}, \dot{q}_{o}, v, \dot{v}\right)
\end{gathered}
$$

where $\theta^{*}$ being the vector of unknown parameters in the system, $Y(q, \dot{q}, v, \dot{v})$ being a known regressor matrix and $F\left(q_{o}, \dot{q}_{o}, v, \dot{v}\right)$ is a known matrix.

We choose the control $\tau(t) \in R^{12}$ such that

$$
E^{T} \tau=Y(q, \dot{q}, v, \dot{v}) \theta^{*}+F\left(q_{o}, \dot{q}_{o}, v, \dot{v}\right)-K_{d} s(t) .
$$

With the chosen control law for the desired nominal performance, the ideal closedloop system becomes

$$
\begin{aligned}
E^{T} D_{m} E \dot{s} & +E^{T}\left(D_{m} \dot{E}+C_{m} E\right) s \\
& +J_{b} D_{o} \dot{s}+J_{b} C_{o} s=0 .
\end{aligned}
$$

For a robotic system without actuator failures, we can perfectly generate the control signal $\tau$, so that the equation (8.12) holds. In this case, we can prove that $s$ is bounded and $s \in L^{2}$, so $\lim _{t \rightarrow \infty} s(t)=0$ as $\dot{s}$ is also bounded. From the definition of $s$, the convergence of $s$ also implies the convergence of the tracking error. 


\subsubsection{Actuator Failure Compensation}

In this subsection, we consider the helicopter landing system, where the uncertainties of actuator failure and parameter uncertainties are known; that is, to design a feedback control signal $\tau_{c}^{*}(t)$ to meet the desired nominal signal $E^{T} \tau(t)=$ $Y(q, \dot{q}, v, \dot{v}) \theta^{*}+J_{b} D_{o} \dot{v}+J_{b} C_{o} v-K_{d} s(t)$ for $\tau=\left(I-\sigma_{(i)}\right) \tau_{c}^{*}+\sigma_{(i)} \bar{\tau}, \forall \sigma_{(i)} \in \Sigma$, so the closed loop system $E^{T} D_{m} E \dot{s}+E^{T}\left(D_{m} \dot{E}+C_{m} E\right) s+J_{b} D_{o} \dot{s}+J_{b} C_{o} s=0$.

The proposed actuator failure compensation scheme chooses the nominal controller structure as a combination of individual controller for each actuator failure pattern as shown in Figure 8.2 as

$$
\tau_{c}^{*}(t)=\sum_{i=0}^{N} \chi_{i}^{*} \tau_{c(i)}^{*}(t)
$$

where $N$ is the number of all possible actuator failure cases and $\chi_{i}^{*}$ for $i=0,1, \ldots, N$ are the indicator functions of the actuator failure: $\chi_{0}^{*}=1$ when there is no actuator failure, $\chi_{i}^{*}=1$ for $i$ actuator failure. The indicator functions $\chi_{i}^{*}=0$ for its noncorresponding cases.

The controller design for each actuator failure pattern can be obtained as follows:

Step 1: Define an intermediate control vector $\tau_{c a}^{*}$ from the control signal $\tau_{c}$ by remove $j^{\text {th }}$ element associated with the failing actuator (we remove the signal associated with the actuator failure because we have no control over the signals when the actuators fail).

For each actuator failure case $\sigma_{(i)}$, lets $\tau_{c}^{*}(t)=\tau_{c(i)}^{*}(t)$ as

$$
\tau_{c(i)}^{*}(t)=\left[\alpha_{1} \tau_{c a(i) 1}^{*}, \alpha_{2} \tau_{c a(i) 2}^{*}, \ldots, \alpha_{12} \tau_{c a(i) 12}^{*}\right]^{T}
$$


where $\alpha_{i}=0$ if the actuator $i$ fails and $\alpha_{i}=1$ otherwise. The intermediate vector signal $\tau_{c a(i)}^{*}$ is then defined as a vector consisting of the non-zero rows of $\tau_{c(i)}^{*}$. From this definition, the control signal $\tau_{c(i)}^{*}$ can be reconstructed by inserting a zero for each column associated with the failing actuator into $\tau_{c a(i)}^{*}$.

Step 2: Formulate the desired control signal structure from the baseline control design. The intermediate control signal can be designed from

$$
\tau_{c a(i)}^{*}(t)=h_{i} \tau_{c b(i)}^{*}(t)
$$

with some chosen matrix function $h_{i} \in R^{\left(12-\bar{m}_{i}\right) \times\left(12-\bar{m}_{i}\right)}$ and $\tau_{c b(i)}^{*}(t) \in R^{\left(12-\bar{m}_{i}\right) \times 1}$ are obtained from

$$
\sum_{j \in \bar{\alpha}_{(i)}} E_{j}^{T} \bar{\tau}_{j}+\bar{E}_{(i)}^{T} h_{i} \tau_{c b(i)}^{*}(t)=Y \theta^{*}+F-K_{d} s(t)
$$

where the submatrix $\bar{E}_{(i)} \in R^{\left(12-\bar{m}_{i}\right) \times 3}$ represents the Jacobian matrix $E$ without the row associated with the failing actuators, $\bar{m}_{i}$ is the number of concurrent actuator failure for each actuator failure case $\sigma_{(i)}$ and $\bar{\alpha}_{(i)}$ is defined as a set of all failing actuator index $j$ such that $\sigma_{(i) j}=1$.

Step 3: Solving for the explicit form of $\tau_{c b(i)}^{*}(t)$ to obtain the desired control signal.

With Assumption 1, it is possible to choose $h_{i}$, such that the solution of $\tau_{c b(i)}^{*}(t)$ exists. In order to obtain a unique solution, we choose the design matrix $h_{i}$ such that $\bar{E}_{(i)}^{T} h_{i}$ is a square matrix and is invertible. 
The explicit form of the $\tau_{c b(i)}^{*}(t)$ can be written as

$$
\tau_{c b(i)}^{*}(t)=K_{i 0}\left(Y \theta^{*}+F-K_{d} s(t)\right)+\sum_{j \in \bar{\alpha}_{(i)}} K_{i j} \bar{\tau}_{j} .
$$

where $K_{i 0} \in R^{3 \times 3}$ and $K_{i j} \in R^{3 \times 1}$ are expressed as

$$
K_{i 0}=\left(\bar{E}_{(i)}^{T} h_{i}\right)^{-1}, K_{i j}=-\left(\bar{E}_{(i)}^{T} h_{i}\right)^{-1} E_{j} .
$$

The proposed nominal control design ensures $E^{T} \tau=E^{T}\left(I-\sigma_{(i)}\right) \tau_{c}^{*}+E^{T} \sigma_{(i)} \bar{\tau}=$ $Y \theta^{*}+F-K_{d} s(t)$, so that the closed loop system $E^{T} D_{m} E \dot{s}+E^{T}\left(D_{m} \dot{E}+C_{m} E\right) s+$ $J_{b} D_{o} \dot{s}+J_{b} C_{o} s=0$ for every actuator failure case.

In this section, we have designed a nominal control law for the system, where all uncertainties in the system are known. In the next section, we will develop an adaptive actuator failure compensation scheme without the knowledge of the actuator failure in addition to parameter uncertainties.

\subsection{Adaptive Failure Compensation Design}

The proposed helicopter landing mechanism needs to operate on various types of terrains under different circumstances, thus some system parameters may be unknown. Additionally, the information regarding the actuator failure, such as the actuator failure values $\bar{\tau}_{(i)}$, actuator failure pattern $\sigma_{(i)}$, or the failing times $t_{(i)}$ is often impossible to obtain. In this section, we proposed an adaptive actuator failure compensation scheme for the helicopter landing system with unknown parameters in addition to uncertain actuator failures.

The system block diagram of the proposed adaptive actuator failure compensation 
scheme is shown in Figure 8.3.

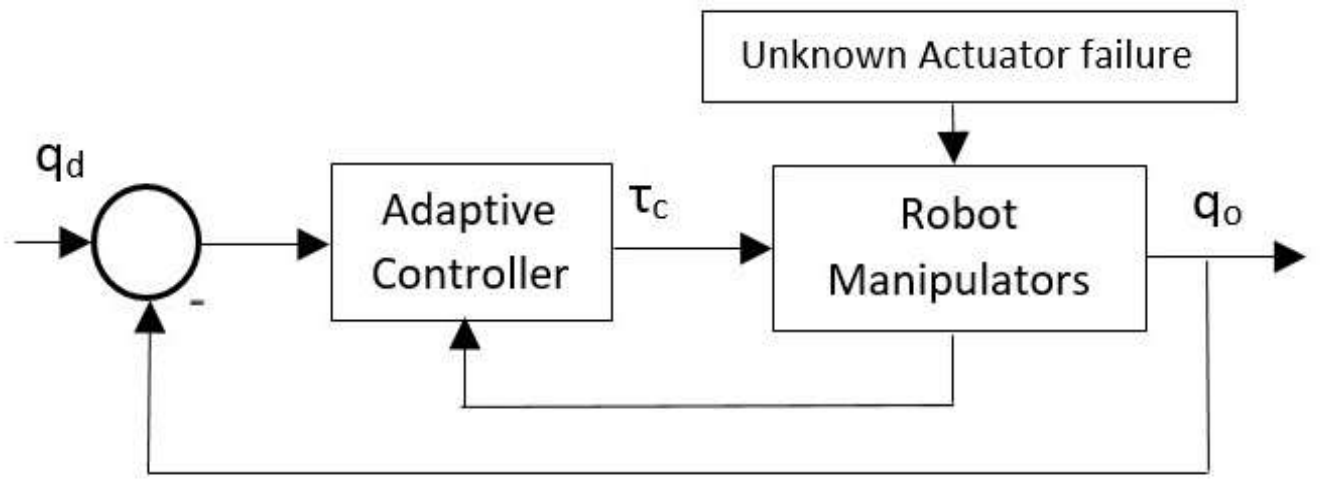

Figure 8.3: Adaptive system Block Diagram.

The control input $\tau_{c}(t)$ is the combination of the parameterize controller structure, which is developed from the nominal controller design in the previous section. Because some parameters in the controller structure are unknown, we develop estimators corresponding to each adaptive controller, the estimators estimate the actuator failure values, actuator failure patterns and the uncertain parameters in the system. In this section, we will first parameterize the nominal controller to obtain a suitable adaptive controller structure, and then we will develop adaptive laws for the system.

\subsubsection{Adaptive Controller Structure}

The adaptive control scheme is developed based on the nominal controller structure (??). Since we do not know the values of $\chi_{i}^{*}, \bar{\tau}_{(i)}$ and $\theta^{*}$, we first design the controller as

$$
\tau_{c}(t)=\sum_{i=0}^{N} \tau_{c \chi(i)}(t)
$$

where $\tau_{c \chi(i)}(t)$ are the estimates of each actuator failure case with the indicator function $\chi_{i}^{*} \tau_{c(i)}^{*}$

To derive each adaptive controller structure $\tau_{c \chi(i)}(t)$, we first define an intermediate 
control signal $\tau_{c \chi a(i)}(t)$, similar to the process in the nominal control design. We consider the adaptive control signal $\tau_{c \chi(i)}(t)=\left[\alpha_{1} \tau_{c \chi(i) 1}, \alpha_{2} \tau_{c \chi(i) 2}, \ldots, \alpha_{12} \tau_{c \chi(i) 12}\right]^{T}$, where $\alpha_{i}=0$ if actuator $i$ fails and $\alpha_{i}=1$ otherwise. Then, we calculate the elements of $\tau_{c \chi(i)}(t)$ from its subvector $\tau_{c \chi a(i)}$. The subvector $\tau_{c \chi a(i)}$ is obtained from the original control signal $\tau_{c \chi(i)}(t)$ without the element associated with the failing actuator for each actuator failure pattern $\sigma_{(i)}$.

To derive the intermediate controller structure $\tau_{c \chi a(i)}$, we consider its nominal version as

$$
\begin{aligned}
\chi_{i}^{*} \tau_{c a(i)}^{*}(t)= & -\chi_{i}^{*} h_{i} K_{i 0} K_{d} s+h_{i} K_{i 0} Y \chi_{i}^{*} \theta^{*} \\
& +\sum_{j \in \bar{\alpha}_{(i)}} \chi_{i}^{*} \bar{\tau}_{j} h_{i} K_{i j} .
\end{aligned}
$$

From the nominal controller structure (8.21), the term $\chi_{i}^{*}, \chi_{i}^{*} \theta^{*}$ and $\chi_{i}^{*} \bar{\tau}_{j}$ are unknown and needs to be parameterized.

In this study, we consider the parameterization of unknown system dynamics and actuator failures as follows:

- The indicator function $\chi_{i}^{*}$ is parameterized as $\operatorname{diag}\left\{\chi_{i 1}, \chi_{i 2}, \ldots, \chi_{i \hat{m}_{i}}\right\}$, where $\hat{m}_{i}=12-\bar{m}_{i}$, the term $\chi_{i j}$ is an estimate of $\chi_{i}^{*}$ with $\chi_{i j}^{*}=\chi_{i}^{*}$ and $\theta_{i j}$ is the estimate of $\chi_{i j}^{*} \theta^{*}$,

- The term $\chi_{i}^{*} \theta^{*}$ associated with the unknown parameters is estimated by $\theta_{i j}=$ $\left[\theta_{i 1}, \ldots, \theta_{i \hat{m}}\right]$ such that

$$
h_{i} K_{i 0} Y \chi_{i}^{*} \theta^{*}=\left[\begin{array}{c}
\eta_{i 1} \theta_{i 1}^{*} \\
\vdots \\
\eta_{i \hat{m}_{i}} \theta_{i \hat{m}_{i}}^{*}
\end{array}\right],
$$


where $\eta_{i}=\left[\eta_{i 1}, \ldots, \eta_{i \hat{m}}\right]^{T}=h_{i} K_{i 0} Y$.

- The actuator failure value $\chi_{i}^{*} \bar{\tau}_{j}$ is parameterize based on the proposed actuator failure model (8.8). The actuator failure value can be modeled as $\bar{\tau}_{j}(t)=\rho_{j}^{* T} f_{a j}(t)$, where $f_{a j}(t)=\left[1, f_{a j 1}(t), \ldots, f_{a j \bar{\rho}_{i}}(t)\right]^{T}$ are known functions corresponding to the failed components, $\rho_{i}^{*}=\left[\bar{\tau}_{j 0}, \bar{\tau}_{j 1}, \ldots, \bar{\tau}_{j \bar{\rho}_{i}}\right]^{T}$ contains the parameter values associated with each actuator failure component and $\bar{\rho}$ is the number of actuator failure components. The term $\chi_{i j}^{*} \rho_{i}^{*}$ is estimated by $\rho_{i(j)}$ such that

$$
\sum_{j \in \bar{\alpha}_{(i)}} \chi_{i}^{*} \bar{\tau}_{j} h_{i} K_{i j}=\sum_{j \in \bar{\alpha}_{(i)}}\left[\begin{array}{c}
\rho_{i(1)}^{* T} f_{a i}(t) \phi_{i 1} \\
\vdots \\
\rho_{i(\hat{m})}^{* T} f_{a i}(t) \phi_{i \hat{m}}
\end{array}\right]
$$

where $\phi_{i}=\left[\phi_{i 1}, \ldots, \phi_{i \hat{m}}\right]^{T}=h_{i} K_{i j}$.

Finally, we can choose the controller $\tau_{c \chi a(i)}(t)$ as

$$
\begin{aligned}
\tau_{c \chi a(i)}(t)= & -\operatorname{diag}\left\{\chi_{i 1}, \chi_{i 2}, \ldots, \chi_{\left.i \hat{m}_{i}\right)}\right\} K_{d} s(t) \\
& +\left[\begin{array}{c}
\eta_{i 1} \theta_{i 1} \\
\vdots \\
\eta_{i \hat{m}_{i}} \theta_{i \hat{m}_{i}}
\end{array}\right]+\sum_{j \in \bar{\alpha}_{(i)}}\left[\begin{array}{c}
\rho_{i(1)}^{T} f_{a i}(t) \phi_{i 1} \\
\vdots \\
\rho_{i(\hat{m})}^{T} f_{a i}(t) \phi_{i \hat{m}}
\end{array}\right]
\end{aligned}
$$

From the intermediate controller structure, we can obtain $\tau_{c \chi(i)}(t)$ by adding the zero columns associated with the failing actuator to $\tau_{c \chi a(i)}(t)$. The adaptive controller structure developed in this section will be used with the adaptive law to ensure that the control objective is met for the system with unknown actuator failures in addition to parameter uncertainties. 


\subsubsection{Adaptive Laws}

In this subsection, we develop adaptive laws for updating the estimated parameters $\chi_{i j}, \rho_{i(j)}, \theta_{i j}$ in the controller structure (8.20). The adaptive laws proposed in this study are developed based on the Lyapunov method to ensure desired closed-loop stability and asymptotic output tracking when applied to the adaptive controller structure.

The adaptive laws for $\chi_{i j}$ with $i=0,1, \ldots, N$ and $j=1,2, \ldots, \hat{m}_{i}$ are chosen as

$$
\dot{\chi}_{i j}=-\gamma_{i j} s^{T} \bar{E}_{(i) j} \mu_{i j}+P_{\chi_{i j}},
$$

where $\bar{E}_{(i) j} \in R^{3 \times 1}$ represents the $j$ column of the submatrix $\bar{E}_{(i)}$ defined for each adaptive controller $\tau_{c \chi(i)}(t), \gamma_{i j}>0$ is the adaptation gain, $\mu_{i}=\left[\mu_{i 1}, \mu_{i 2}, \ldots, \mu_{i n}\right]^{T}=$ $-h_{i} K_{i 0} K_{d} s(t), P_{\chi i j}$ is the projection function, $h_{i}$ and $K_{i 0}$ are the matrices obtained from the nominal design process for each actuator failure case.

The adaptive laws for $\rho_{i(j)}=\left[\rho_{i(j)(1)}, \ldots, \rho_{i(j)\left(n_{\tau}\right)}\right]^{T}$ with with $i=0,1, \ldots, N$ and $j=1,2, \ldots, \hat{m}_{i}$ are chosen as

$$
\dot{\rho}_{i(j)}=-\Gamma_{(i j)} f_{a j} s^{T} \bar{E}_{(i) j} \phi_{i j}+P_{\rho_{i(j)}},
$$

where $P_{\rho i(j)}$ is the projection function and $\Gamma_{(i j)}=\Gamma_{(i j)}^{T}>0$ are the adaptation gain matrices.

The adaptive laws for $\theta_{i j}$ with $i=0,1, \ldots, N$ and $j=1,2, \ldots, \hat{m}_{i}$ is

$$
\dot{\theta}_{i j}=-\beta_{i j} s^{T} \bar{E}_{(i) j} \eta_{i j}+P_{\theta_{i}},
$$

where $P_{\theta_{i}}$ is the projection function, $\eta_{i}=\left[\eta_{i 1}, \eta_{i 2}, \ldots, \eta_{i n}\right]^{T}=h_{i} K_{i 0} Y, \beta_{i j}=\beta_{i j}^{T}>0$ 
are the adaptation gain matrices.

Remark 8.3: The adaptive laws proposed in this paper uses parameter projection designs for $P_{\chi_{i j}}, P_{\rho_{i(j)}}$ and $P_{\theta_{i}}$. The parameter projection utilizes the information of the parameter regions based on the physical properties of the system $0 \leq \chi_{i}^{*} \leq 1$, $\theta_{j}^{* a} \leq \theta_{i j}^{*} \leq \theta_{j}^{* b}$ and $\rho_{i k}^{* a} \leq \rho_{i k}^{*} \leq \rho_{i k}^{* b} . \rho_{i k}^{* a}$ and $\rho_{i k}^{* b}$ being the upper and lower bounds of the components $\rho_{i k}^{*}$ of $\rho_{i}^{*} . \theta_{j}^{* a}$ and $\theta_{j}^{* b}$ being the upper and lower bounds of each unknown parameter in the system $\theta^{*}$.

This choice of the projection functions $\varrho_{\chi}(t), \varrho_{\theta}(t)$ and $\varrho_{\rho}(t)$ ensures the following properties $\left(\chi_{i j}-\chi_{i j}^{*}\right) P_{\chi_{i j}} \leq 0,\left(\theta_{i}-\chi_{i}^{*} \theta^{*}\right) P_{\theta}(t) \leq 0,\left(\rho_{i(j k)}-\chi_{i j}^{*} \rho_{i k}^{*}\right) P_{\rho_{i(j k)}} \leq 0$ which guarantees the boundedness of the estimated parameters.

\subsubsection{Performance Analysis}

The proposed actuator failure compensation scheme can ensure the stability and tracking of the helicopter landing system with parameter uncertainties subjected to unknown actuator failures as shown by the following theorem.

Theorem 8.1. The adaptive actuator failure compensation scheme with the adaptive controller structure (8.20) updated by the adaptive laws (8.25) - (8.27), when applied to proposed helicopter system model (8.6), guarantees the closed-loop signal boundedness and asymptotic output tracking: $\lim _{t \rightarrow \infty} e(t)=0$, despites parameter uncertainties in the system in addition to unknown actuator failure index, failure times and failure values.

Proof: Define $\left(T_{i}, T_{i+1}\right), i=0,1,2, \ldots, p$ with $T_{0}=0, T_{p}=\infty$ as the time intervals on which the actuator failure pattern is fixed. To guarantee the performance of the system, we need to show that there exists a Lyapunov function for every time interval 
$\left(T_{i}, T_{i+1}\right)$, where $T_{i}$ is the time that actuator failure pattern changes. The Lyapunov function $V_{i}$ corresponding to each actuator failure pattern $\sigma_{i}$ is

$$
\begin{gathered}
V_{i}=\frac{1}{2} s^{T}\left(E^{T} D_{m} E+J_{b} D_{o}\right) s+\frac{1}{2} \sum_{i=0}^{N} \sum_{j=1}^{\hat{m}} \tilde{\chi}_{i j}^{2} \gamma_{i j}^{-1} \\
+\frac{1}{2}\left(\sum_{i=0}^{N} \sum_{j=1}^{\hat{m}} \tilde{\theta}_{i j}^{T} \beta_{i j}^{-1} \tilde{\theta}_{i j}+\sum_{i=0}^{N} \sum_{j=1}^{\hat{m}} \tilde{\rho}_{i(j)}^{T} \Gamma_{i j}^{-1} \tilde{\rho}_{i(j)}-\sum_{i=0}^{N} \sum_{k \in \sigma_{i}} \tilde{\chi}_{i k}^{2} \gamma_{i k}^{-1}\right. \\
\left.-\sum_{i=0}^{N} \sum_{k \in \sigma_{i}} \tilde{\theta}_{i k_{1}}^{T} \beta_{i k}^{-1} \tilde{\theta}_{i k}-\sum_{i=0}^{N} \sum_{k \in \sigma_{i}} \tilde{\rho}_{i(k)}^{T} \Gamma_{i k_{1}}^{-1} \tilde{\rho}_{i(k)}\right) .
\end{gathered}
$$

Using the properties of the parameter projection, the time derivative of the Lyapunov function $V_{i}$ is

$$
\dot{V}_{i} \leq-s^{T} K_{d} s \leq 0 .
$$

With the Lyapunov function, $s(t) \in L^{\infty} \bigcap L^{2}$ and all signals in the system are bounded, and so is $\dot{s}$; based on the Barbalat lemma, we can conclude that $\lim _{t \rightarrow \infty} s(t)=$ 0 and $\lim _{t \rightarrow \infty} e(t)=0$.

Because the state of the system in any time interval $\left(T_{i}, T_{i+1}\right)$ must belong to an actuator failure pattern $\sigma_{i} \in \Sigma$, where $\Sigma$ is the set of all possible actuator failure petterns including the no failure case. In this study, we have designed the controller for all actuator failure pattern $\sigma_{i}$, so that we have a Lyapunov function for each time interval $\left(T_{i}, T_{i+1}\right)$. As a result, we can guarantee the performance of the system at all time $t \in(0, \infty)$.

\subsection{Simulation Study}

In order to guarantee the performance of the proposed adaptive actuator failure compensation scheme, we design an adaptive actuator failure compensation for landing of a helicopter with robotic legs. In this case, the helicopter is supported by six 
robot manipulators, which are used to support and maintain the orientation of the helicopter body.

\subsubsection{Simulation Conditions}

The dynamic of each robot manipulator can be written as presented in (8.1). The derivation of the specific two-link planar robot manipulator can be found in [19]. In the simulation study, we assign the parameter as follows: The mass of each link $m_{i}=10 \mathrm{~kg}, I_{i}=5 \mathrm{~kg} \cdot \mathrm{m}^{2}$ the length of each link $l_{i}=1 \mathrm{~m}$ for $i=1,2, \ldots, 12$, where actuator $1,2, \ldots, 6$ are the upper part and actuator $7,8, \ldots, 12$ are the lower part of manipulator $1,2, \ldots, 6$ respectively. With the helicopter facing the $x$ axis and the center of the helicopter body is at located at $(0,0,1)$ initially, the base position of each manipulator is located at $(1,0,1),(0.866,0.5,1),(0.866,-0.5,1),(-1,0,1),(-0.866,0.5,1$ ),$(-0.866,-0.5,1)$ respectively.

In this study, we model the dynamic of the helicopter body as a rigid mass with unknown parameters as shown in (8.3). The dynamic model of the helicopter can be estimated by

$$
\begin{aligned}
I_{r} F_{r}(t) \ddot{\theta}_{r} & =0.866\left(\tau_{02}+\tau_{03}-\tau_{05}-\tau_{06}\right) \\
I_{p} F_{p}(t) \ddot{\theta}_{p} & =\tau_{01}-\tau_{04}+0.5\left(\tau_{02}+\tau_{06}-\tau_{03}-\tau_{05}\right) \\
M_{c} F_{m}(t) \ddot{h} & =\sum_{i=1}^{6} \tau_{01}-M_{c} g
\end{aligned}
$$

where the height $h$, roll angle $\theta_{r}$ and pitch angle $\theta_{p}$ are the output of the system needed for a safe landing, the mass and moment of inertia of the helicopter $M_{c}=$ $500 \mathrm{~kg}, I_{c}=100 \mathrm{~kg} \cdot \mathrm{m}^{2}, I_{p}=100 \mathrm{~kg} \cdot \mathrm{m}^{2}$ are unknown to the controller, $F_{r}(t), F_{p}(t), F_{c}(t)$ are known functions from the flight dynamic and $\tau_{0 i}$ is the perpendicular force of the manipulator to the base of the helicopter body. 


\subsubsection{Simulation Results}

In this section, we show the results of our study. In this case, we only consider simple cases of simulation with constant reference input and constant actuator failure. The reference of the output for $q_{o}=\left[h, \theta_{r}, \theta_{p}\right]$ is $q_{d}=[1,0,0]$.

In this simulation, we use the complete failure of actuators $\tau_{i}=0$. We let actuator $q_{1}, q_{3}$, and $q_{5}$ fail concurrently at time $t \geq 50 \mathrm{~s}$ :

(i) No actuator failure case: $\tau(t)=\tau_{c}(t)$ for $0 \leq t<50 \mathrm{~s}$,

(ii) Actuator $q_{1}, q_{3}$, and $q_{5}$ failure case: $\tau_{1}=\tau_{3}=\tau_{5}=0, M c=0.9 M c^{*}$ at $t>50 \mathrm{~s}$

In this case, the simulation results show the output of the system in Figure 8.4.
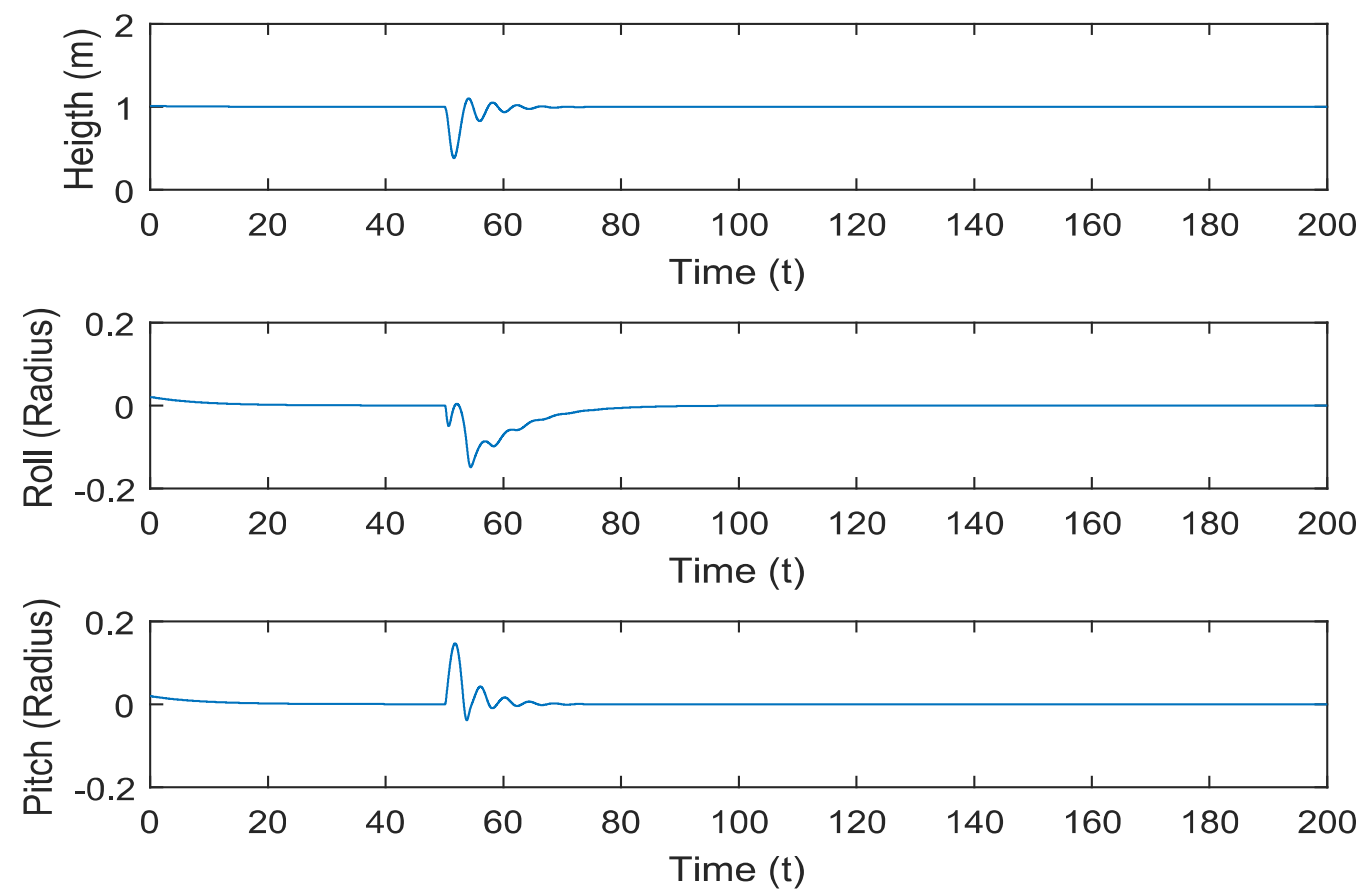

Figure 8.4: The output of the system $q_{o}$ for $q_{d}=[1,0,0]$. 

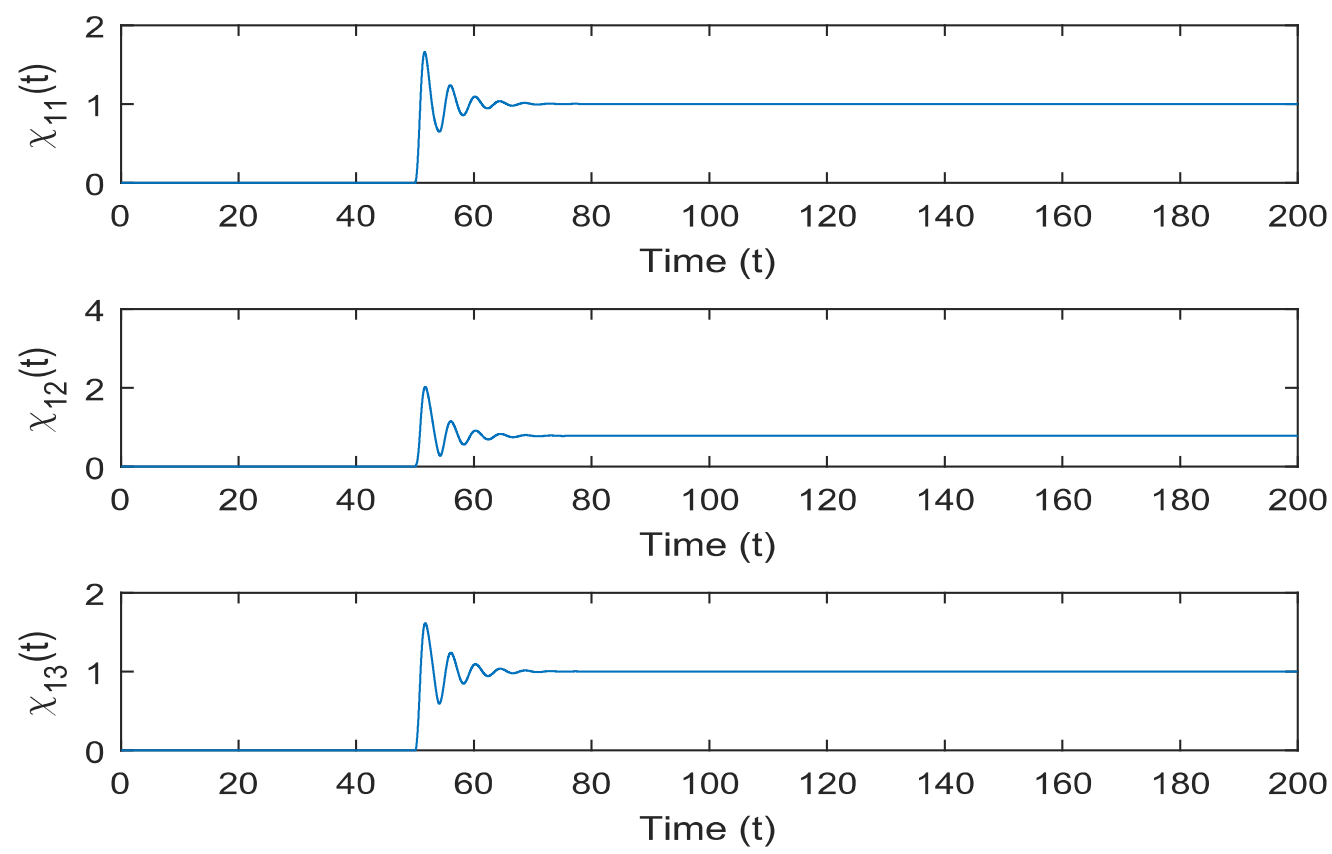

Figure 8.5: The estimate of the actuator failure pattern $\chi_{1}$ (no actuator failure case).
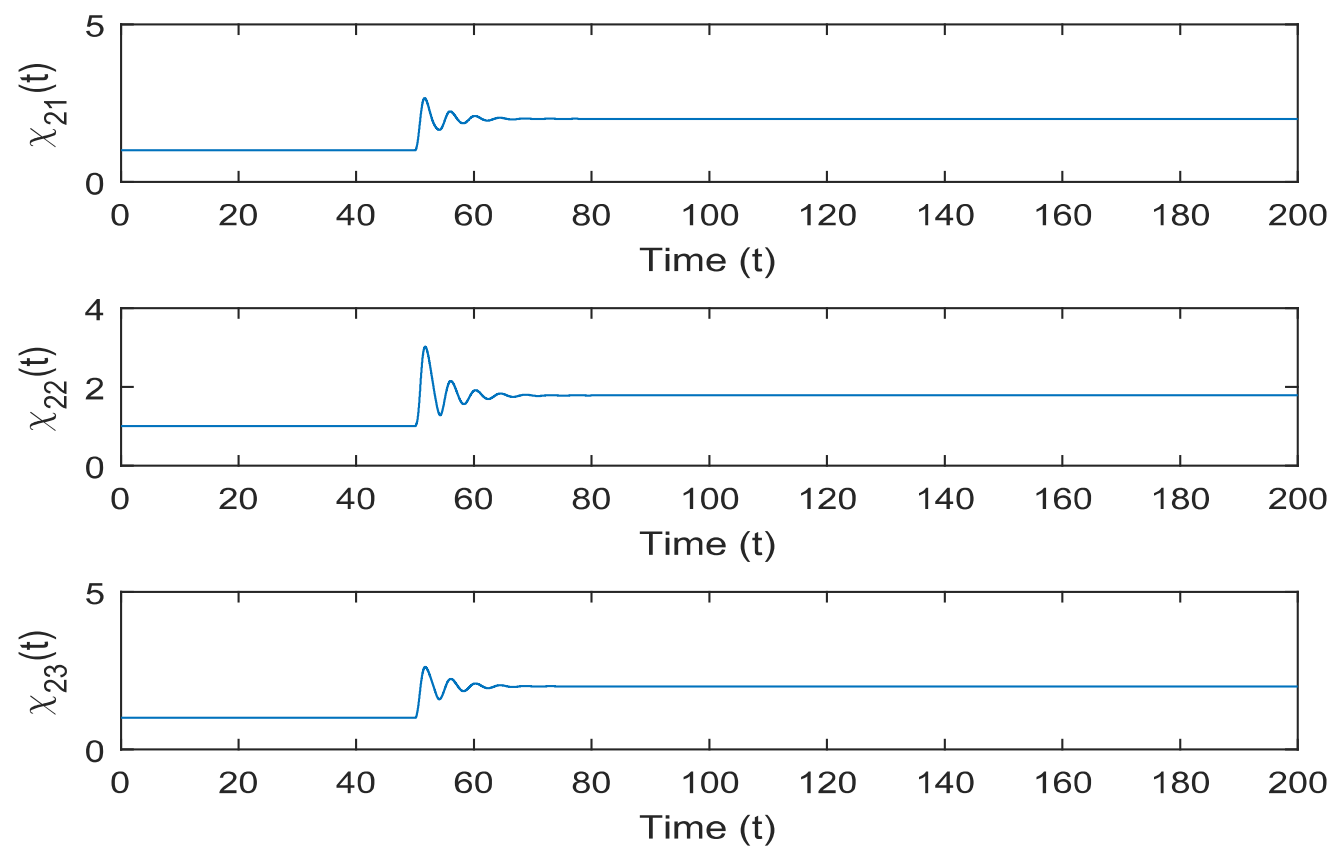

Figure 8.6: The estimate of the actuator failure pattern $\chi_{2}\left(\tau_{1}\right.$ failure case). 
CHAPTER 8. DESIGN FOR LANDING OF A HELICOPTER WITH ROBOTIC
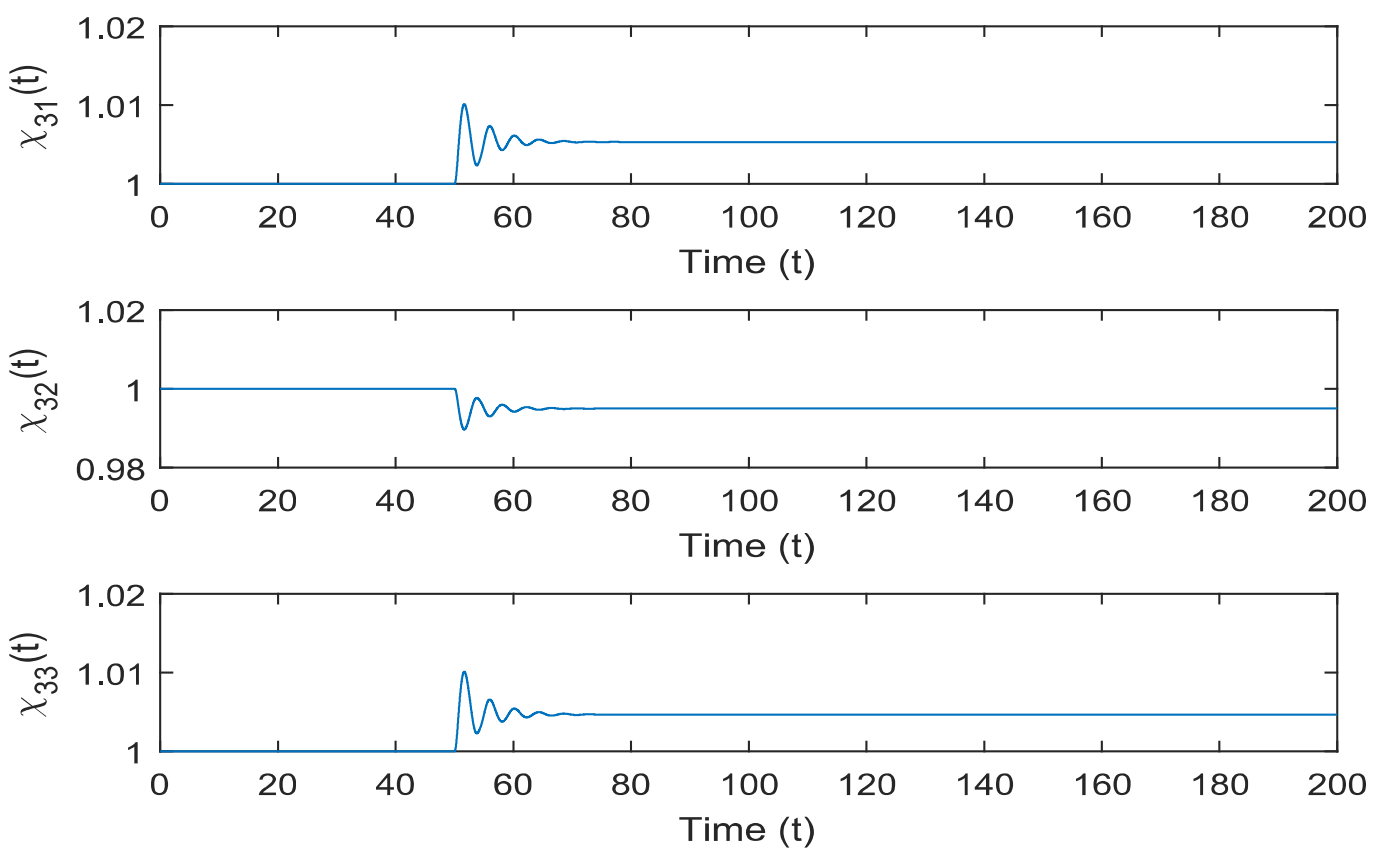

Figure 8.7: The estimate of the actuator failure pattern $\chi_{2}\left(\tau_{1}, \tau_{3}\right.$ and $\tau_{5}$ failures $)$.

Figure $8.5-8.7$ show the estimates of $\chi_{1}, \chi_{2}, \chi_{3}$, which represent the estimates of the actuator failure patternf (the nofailure case, $\tau_{1}$ actuator failure case and $\tau_{1}, \tau_{3}, \tau_{5}$ failure case repectively). From this we can see that, the system does not switch to a specific controller as in the nominal case, but the system use the combination of three controllers to control the system.

The results confirm that the adaptive actuator failure compensation scheme can guarantee that the tracking error of the system goes to zero, thus able to maintain the orientation of the helicopter. 


\subsection{Conclusions}

In this chapter, we have studied the adaptive actuator failure compensation scheme for the landing of a new helicopter with robotic legs subject to uncertain actuator failures. The adaptive control design uses an integration of multiple individual failure compensators and direct adaptation to handle actuator failure and parameter uncertainties in the system. The proposed algorithm guarantees that a desirable closed-loop stability and asymptotic tracking property can be achieved, despite uncertain actuator failures in addition to parameter uncertainty. The simulation result verified the performance of the proposed algorithm when applied to the helicopter model subjected to uncertain actuator failures. 


\section{Chapter 9}

\section{Conclusion and Future Works}

This research develops an adaptive actuator failure compensation framework of nonlinear multi-input multi-output robotic systems subject to uncertain actuator failures with parameter uncertainties in addition to actuator failures. The research shows a complete design methodology for developing an actuator failure compensation scheme for robotic systems such as cooperative manipulator system and parallel structure robotic system. The adaptive actuator failure compensation framework guarantees desired closed-loop stability and asymptotic output tracking, despite actuator failures whose patterns, times and values are all unknown. Simulation results are presented to verify the desired adaptive actuator failure compensation control performances for multiple types of robotic systems from the theoretical cooperative manipulator benchmark system to the landing of a helicopter with robotic legs. From the simulation results we can also see that the algorithm can work with both actuator degradation cases as well as the complete failure cases.

\section{$9.1 \quad$ Future Works}

The adaptive actuator failure compensation framework developed in this study ensures the performance of the system subject to unknown actuator failure. Although the control framework can greatly enhance the robustness of the system, it is still in 
the early stage of development. Due to the lack of advanced researches related to actuator failure compensation in robotic system, there are still many open problems that need to be solved. In this section, we discuss a few open problems related to the adaptive actuator failure compensation within the robotic field of study.

Actuator Dynamic: In our study, we assume that the torque of each actuator in the system $\tau(t)$ can be produced precisely. However, the assumption may not hold in practice. Due to the dynamic of the motor the actual actuator output $\tau(t)$ may be different from the desired control signal. This error could deteriorate the performance of the system, especially for the system that required high precision and operate with a relatively high velocity. The study of the adaptive actuator failure compensation for robotic system with actuator dynamic is an important topic that could further enhance the performance of the control design.

Flexible Joint Robot Manipulator: Another open problem related to the dynamic model of the robotic system is the consideration of flexible joint robot. The transmission mechanism in many industrial robots contains flexible joint components. The study of robotic systems with flexible joint could further increase the performance of the control design.

Sensor Faults: The adaptive actuator failure compensation framework developed in this study only consider the failure in the actuators. However, the faults in robotic system can also originate from the problem of the sensors in the system.F The study of sensor fault is one of the most challenging open problems related to the faulttolerant control of robotic systems. 
Control Optimization Methods: As mentioned previously, the adaptive actuator failure compensation framework developed in this study is still is a very early stage. Although the proposed control framework can guarantee the desired performance of the system, there are many aspects of the controller that could potentially be improved. From the recent literatures, an adaptive control of a system could be optimized based on a certain cost function using optimization techniques such as the multi-mode adaptive control. However, the nonlinearity and uncertainties in robotic system prevent such method from being used. When the parameters in the system are unknown, it affects the calculation of the cost function. Thus, the current optimization technique cannot be directly applied to our framework. The research related to optimization techniques for the adaptive actuator failure compensation framework could potentially improve the performance of the actuator failure compensation framework. 


\section{Bibliography}

[1] M. Dede, S. Tosunoglu, "Fault-tolerant teleoperation systems design," Industrial Robot: An International Journal, Vol. 33/5, pp. 365-372, 2006.

[2] K. Ohno, S. Kawatsuma, T. Okada, E. Takeuchi, K. Higashi, S. Tadokoro, "Robotic control vehicle for measuring radiation in Fukushima Daiichi Nuclear Power Plant," Proceedings of the IEEE International Symposium on Safety, Security, and Rescue Robotics (SSRR), pp. 38 - 43, November, 2011.

[3] K. Jones, "Special Weapons Observation Remote recon Direct Action System (SWORDS)," In Platform Innovations and System Integration for Unmanned Air, Land and Sea Vehicles, pp. 36:1-8. 2007.

[4] S. Weinberger, "Armed Robots Still in Iraq, But Grounded," April 15, 2008. Available at http://www.wired.com/dangerroom/2008/04/armed-robots-st/.

[5] G. Liu, "Control of Robot Manipulators with Consideration of Actuator Performance Degradation and Failures," Proceedings of the 2001 IEEE International Conference on Robotics and Automation, Seoul, Korea, May 2001.

[6] M. Ozkan, G. Kirlik, O. Parlaktuna, A. Yufka and A. Yazici, "A Multi-Robot Control Architecture for Fault-Tolerant Sensor-Based Coverage," International Journal of Advanced Robotic Systems, Vol. 7, No. 1, pp. 067-074, 2010 
[7] A.H. Al-Bayati, H. Wang, "A Robust New Nonlinear Fault Tolerant Control for Robot via an Observer," Proceedings of the 8th World Congress on Intelligent Control and Automation, June 2011.

[8] M. Ji, N. Sarkar, "Supervisory Fault Adaptive Control of a Mobile Robot and Its Application in Sensor-Fault Accommodation," IEEE Transactions on Robotics, Vol. 23, No. 1, February, 2007.

[9] X. Zhang, M. M. Polycarpou, and T. Parisini, "Fault diagnosis of a class of nonlinear uncertain systems with Lipschitz nonlinearities using adaptive estimation, Automatica, vol 46, no. 2, 2010, pp. 290-299.

[10] Y. Diao, K. M. Passino, "Stable fault-tolerant adaptive fuzzy/neural control for turbine engine, IEEE Trans. on Control systems Technology, vol. 9, no. 3, 2001, pp. $494-509$.

[11] B. Jiang, Z. Gao, P. Shi, Y. Xu, "Adaptive fault-tolerant tracking control of nearspace vehicle using takagi-sugeno fuzzy models, IEEE Trans. on fuzzy systems. vol. 18, no. 5, 2010, pp. 1000-1007.

[12] C. Axenie, D. Cernega, "Adaptive Sliding Mode Controller Design for Mobile Robot Fault Tolerant Control. Introducing ARTEMIC," 19th International Workshop on Robotics in Alpe-Adria-Danube Region - RAAD 2010, Budapest, Hungary, June 23-25, 2010.

[13] L. Wu, J. Lam and T. Parisini, "Sliding mode control of switched hybrid systems with time-varying delay, Internation Journal of Adaptive Control and Signal Processing, vol 22, no. 1, 2008, pp. 990-931. 
[14] Defense Advanced Research Projects Agency, "Robotic Landing Gear Could Enable Future Helicopters to Take Off and Land Almost Anywhere," Retrieved from http://www.darpa.mil/news-events/2015-09-10.

[15] J. J. Craig, Introduction to Robotics, New York, Addison-Wesley, 1986.

[16] F. L. Lewis, C. T. Abdallah, and D. M. Dawson, Control of Robot Manipulators, NewYork, Macmillan, 1993.

[17] J. J. Craig, P. Hsu, S.S. Sastry, "Adaptive control of mechanical manipulators," IEEE International Conference on Robotics and Automation, Vol.3, pp.190-195, 1986.

[18] J. J. E. Slotine, and W. Li, "Adaptive manipulator control: A case study," IEEE Transactions on Automatic Control, Vol. 33, No.11, pp. 995-1003, 1988.

[19] R. Kelly, and R. Carelli, "Unified approach to adaptive control of robotic manipulators," IEEE Conference on Decision and Control, pp. 1598-1603, 1988.

[20] J. W. F. Cheung, Y.S. Hung, "Robust learning control of a high precision planar parallel manipulator',' Mechatronicsl, vol. 19, no. 1, pp. 42-55, 2009.

[21] W. Khalil, O. Ibrahim, "General Solution for the Dynamic Modeling of Parallel Robots',' Journal of Intelligent and Robotic Systemsl, vol. 49, no. 1, pp. 19-37, 2007.

[22] B. Dasgupta and T. S. Mruthyunjaya, "A Newton-Euler Formulation for the Inverse DYnamics of the Stewart Platform Manipulator," Mechanism and Machine Theory, vol. 33, no. 8, pp. 1135-1152, 1998.

[23] K. Harib and K. Srinivasan, "Kinematic and dynamic analysis of Stewart platform-based machine tool structures," Robotica, Vol. 21, Issue 5, October, 2003. 
[24] M. Galicki, "The structure of time-optimal controls for kinematically redundant manipulators with end-effector path constraints, IEEE International Conference on Robotics and Automation, Vol.1, pp.101-106, 1998.

[25] G. Liu and Z. Li, "A Unified Geometric Approach to Modeling and Control of Constrained Mechanical Systems" IEEE transaction on Robotics and Automation, vol.18, no. 4, pp.574-587, 2002.

[26] M. W. Spong and M. Vidyasagar, Robot Dynamics and Control, John Wiley \& Sons, New York, 1989.

[27] Y. X. Zhang, S. Cong, W.W. Shang, Z. X. Li and S.L. Jiang, "Modeling, Identification and Control of a Redundant Planar 2-DOF Parallel Manipulator," International Journal of Control, Automation, and Systems, vol. 5, no. 5, pp. 559-569, 2007.

[28] H. Chaoui, P. Sicard, W. Gueaieb, "ANN-Based Adaptive Control of Robotic Manipulators With Friction and Joint Elasticity," IEEE Transactions on Industrial Electronics, Vol.56, No.8, pp. 3174-3187, 2009.

[29] Y. Liu, X. Tang, and G. Tao, "Adaptive failure compensation control of autonomous robotic systems: Application to a precision pointing Hexapod," AIAA Infotech@Aerospace Conference, VA, 2005.

[30] G. Liu, "Control of Robot Manipulators with Consideration of Actuator Performance Degradation and Failures," IEEE International Conference on Robotics and Automation, Vol. 3, pp.2566-2571, 2001.

[31] J. H. Shin, J. J. Lee, "Fault detection and robust fault recovery control for robot manipulators with actuator failures," IEEE International Conference on Robotics and Automation, Vol.2, pp.861-866, 1999. 
[32] W. E. Dixon, I. D. Walker, D. M. Dawson and J. P. Hartranft, "Fault detection for robot manipulators with parametric uncertainty: A prediction error based approach, IEEE International Conference on Robotics and Automation, Vol.4, pp. 3628-3634, 2000.

[33] A. Cristofaro, M. M. Polycarpou and T. A. Johansen, "Fault diagnosis and faulttolerant control allocation for a class of nonlinear systems with redundant inputs," 54th IEEE Conference on Decision and Control, pp. 5117-5123, 2015.

[34] C. Tan, X, Yao, G.Tao, and R. Qi, "A multiple-model based adaptive actuator failure compensation scheme for control of Near-space Vehicles," Proceedings of AIAAInfotech@Aerospace Conference, 2005.

[35] T. Rugthum and G. Tao "An Adaptive Actuator Failure Compensation Scheme for A Cooperative Manipulator System", Proceedings of the 2014 American Control Conference, pp. 1951-1956, USA, 2014.

[36] J. Huang; Y. Wang; T. Fukuda, "Set-Membership-Based Fault Detection and Isolation for Robotic Assembly of Electrical Connectors," IEEE Transactions on Automation Science and Engineering, vol.PP, no.99, pp.1-12, 2016.

[37] D. Crestani, K. Godary-Dejean, L. Lapierre, "Enhancing fault tolerance of autonomous mobile robots," Robotics and Autonomous Systems, vol. 68, pp. 140-155, June 2015.

[38] J. Chen, R. Ling, H. Ni, and D. Zhang, "Distributed Fault Estimation of Nonlinear Networked Systems: Application to Robotic Manipulator," Mathematical Problems in Engineering, vol. 2014, Article ID 121636, 2014. 
[39] A.S. Rezazadeh, H.R. Koofigar, and S. J. Hosseinnia, "Adaptive fault detection and isolation for a class of robot manipulators with time-varying perturbation," Mech Sci Technol vol. 29: 4901. doi:10.1007/s12206-015-1038-5, 2015.

[40] M. S. Khireddine, K. Chafaa, N. Slimane and A. Boutarfa, "Fault diagnosis in robotic manipulators using artificial neural networks and fuzzy logic," World Congress on Computer Applications and Information Systems, pp. 1-6. Hammamet, 2014.

[41] C.R. Burns, V. Fuelling, G.K. Toth, and A. Patel, "A Fault reaction fault isolation and graceful degradation in a robotic system," Google Patents, United States Patent Application 20150112481, 2015.

[42] V. Stoian and E. Bobasu, "Control algorithm for a cooperative robotic system in fault conditions," 12th International Conference on Informatics in Control, Automation and Robotics, pp. 281-288, Colmar 2015.

[43] Y. Liu, Z. Yang and Y. Lin, "Adaptive failure compensation of hysteretic actuators for a class of nonlinear systems via output feedback", Int. J. Adapt. Control Signal Process, vol. 30, pp.1333-1354, 2016.

[44] D. Ye, J. H. Park and Q.Y. Fan, "Adaptive robust actuator fault compensation for linear systems using a novel fault estimation mechanism," Int. J. Robust Nonlinear Control, vol. 26, pp.1597-1614, 2016

[45] - X. Wu, S. Zhang, E.J. Kampen, Q. Chu and W. Shuang, "Optimal adaptive compensation control for a class of MIMO nonlinear systems with actuator failures," UKACC 11th International Conference on Control, pp. 1-6, Belfast, 2016. 
[46] L. Y. Wen, G. Tao, H. Yang and F. Y. Chen, "Adaptive LQ control based actuator failure compensation," Proceedings of 2016 American Control Conference, pp. 2011-2016, Boston, USA, July 6-8, 2016.

[47] C. Tan, G. Tao and H. Yang, "A multiple-model MRAC scheme for multivariable systems with matching uncertainties," Proceedings of the 26th Chinese Process Control Conference, pp. 217-230, Nanchang, China, July 31-August 2, 2015.

[48] Y. J. Ma, B. Jiang and G. Tao, "A new multiple-model adaptive actuator failure compensation scheme for a class of nonlinear MIMO systems," Proceedings of the 34th Chinese Control Conference, pp. 6274-6279, HangZhou, China, July 28-30, 2015 .

[49] J. Cazalilla, M. Valls a, V. Mata, M. Daz-Rodrguez, A. Valera, "Adaptive control of a 3-DOF parallel manipulator considering payload handling and relevant parameter models" "Robotics and Computer-Integrated Manufacturing ", vol. 30, no.5, pp. 468 - 477, 2014.

[50] W. Khalil, O. Ibrahim, "General Solution for the Dynamic Modeling of Parallel Robots', Journal of Intelligent and Robotic Systemsl, vol. 49, no. 1, pp. 19-37, 2007.

[51] B. Dasgupta and T. S. Mruthyunjaya, "A Newton-Euler Formulation for the Inverse DYnamics of the Stewart Platform Manipulator," Mechanism and Machine Theory, vol. 33, no. 8, pp. 1135-1152, 1998.

[52] K. Harib and K. Srinivasan, "Kinematic and dynamic analysis of Stewart platform-based machine tool structures," Robotica, Vol. 21, Issue 5, October, 2003. 
[53] M. Galicki, "The structure of time-optimal controls for kinematically redundant manipulators with end-effector path constraints, IEEE International Conference on Robotics and Automation, Vol.1, pp.101-106, 1998.

[54] S. Moe, A. R. Teel, G. Antonelli and K. Y. Pettersen, "Stability analysis for set-based control within the singularity-robust multiple task-priority inverse kinematics framework," 54th IEEE Conference on Decision and Control, pp. 171-178, 2015.

[55] E. F. Kececi, X. Tang, and G. Tao, "Adaptive failure compensation control of autonomous robotic systems: Application to a precision pointing Hexapod," AIAAInfotech@Aerospace Conference, VA, 2005.

[56] T. Rugthum and G. Tao "An Adaptive Actuator Failure Compensation Scheme for A Cooperative Manipulator System", Proceedings of the 2014 American Control Conference, pp. 1951-1956, USA, 2014.

[57] X. L. Yao, G. Tao, R. Y. Qi and B. Jiang, "An adaptive actuator failure compensation scheme for an attitude dynamic model of near space vehicles," Proceedings of the 2012 American Control Conference, pp. 368-373, Montreal, Canada, June 2012.

[58] Y. Lui, X. Tang, and G. Tao, "Adaptive failure compensation control of autonomous robotic systems: Application to a precision pointing hexapod," Proceedings of AIAA Infotech@Aerospace Conference, Arlington, VA, 2005.

[59] J. H. Jean, L. C. Fu, "An Adaptive Control Scheme for Coordinated Multimanipulator Systems," IEEE Transactions on Robotics and Automation, Vol. 9, No2, April 1993. 
[60] F. Kececi, X. Tang, G. Tao, "Adaptive actuator failure compensation for redundant manipulator," Robotica, Vol. 27, Issue 1. January, 2009.

[61] M.P. Lazarevic, Mathematical Modeling and Control of Redundant Robotic Manipulators using Biological Analog, Mechanics Automatic Control and Robotics, Vol. 3, No 11, 201, pp. 285-294.

[62] X. Tang, G. Tao, S.M. Joshi, "Adaptive actuator failure compensation for nonlinear MIMO systems with an aircraft control application," Automatica, Vol 43, March 2007, pp. 1869-1883.

[63] M. Honegger, A. Codourey, E. Burdet, "Adaptive Control of the Hexaglide, a 6 dof Parallel Manipulator," Proceedings of the 1997 IEEE International Conference on Robotics and Automation, Albuquerque, New Mexico, April 1997.

[64] G. Yang, I.M. Chen, W.K. Lim, S.H. Yeo, "Design and Kinematic Analysis of Modular Reconfigurable Parallel Robots," Proceedings of the I999 IEEE International Conference on Robotics and Automation, Michigan, May 1999.

[65] H. Yang, B. Jiang, M. Staroswiecki, "Supervisory fault tolerant control for a class of uncertain nonlinear systems," Automatica, Vol 45, June 2009, pp. 2319-2324.

[66] S.S. Ge., J. Zhang, T.H. Lee, "Adaptive MNN control for a class of non-affine NARMAX systems with disturbances," Systems and Control Letters, Vol 53, 2004, pp. $1-12$.

[67] G. Paviglianiti, F. Pierri, F. Caccavale, M. Mattei, "Robust fault detection and isolation for proprioceptive sensors of robot manipulators," Mechatronics, Vol. 20, 2010, pp. 162-170. 
[68] B. Jiang, M. Staroswiecki, V. Cocquempot, "Fault Accommodation for Nonlinear Dynamic Systems," IEEE Transections on Automatic Control, Vol. 51, No. 9, September 2006, pp. 1587-1583.

[69] S.N. Huang, K.K. Tan, "Fault Detection, Isolation, and Accommodation Control in Robotic Systems," IEEE Transections on Automation and Engineering, Vol. 5, No. 3, July 2008, pp. 480-489.

[70] X. Zhang, L.F. Ramos, K. Rattan, "Adaptive Fault-Tolerant Fuzzy Control of Robotic Systems," The 28th North American Fuzzy Information Processing Society Annual Conference, Ohio, USA, June 14, 2009.

[71] G. Tao, S. Chen, S.M. Joshi, "An Adaptive Actuator Failure Compensation Controller Using Output Feedback," IEEE Transections on Automatic Control, Vol. 47, No. 3, March 2002, pp. 506-511.

[72] C. Li, "Adaptive and Robust Composite Control of Coordinated Motion of Space Robot System with Prismatic Joint," Proceeding of the $4^{\text {th }}$ World Congress on Intelligent Control and Automation. Shanghai, China, June 2002.

[73] X. Bai, J.D. Turner, J.L. Junkins, "AIAA Astrodynamics Specialist Conference and Exhibit," Keystone, Colorado, August 2006.

[74] J. Guo, G. Tao, Y. Liu, "A multivariable MRAC scheme with application to a nonlinear aircraft model," Automatica, Vol. 47, 2011, pp. 804-812.

[75] Y. Liu, G. Tao, S.M. Joshi, "Modeling and Model Reference Adaptive Control of Aircraft with Asymmetric Damage," Journal of Guidance Control and Dynamics, Vol. 33, No. 5, September 2010. 
[76] A.A. Maciejewski, "Fault Tolerant Properties of Kinematically Redundant Manipulators," IEEE Conference on Robotics and Automation, pp. 638-642, Cincinnati, OH, May 1990.

[77] S.T. Mondschein, G. Tao, "Adaptive Actuator Nonlinearity Compensation and Disturbance Rejection with an Aircraft Application," American Control Conference, San Francisco, CA, USA, June 2011.

[78] H. Xu, M. Mirmirani, "Robust Adaptive Sliding Control for A Class of MIMO Nonlinear System," Proceedings of the 2001 AIAA Guidance, Navigation, and Control Conference, Canada, August 2002.

[79] C. Bonivento, L. Gentili, A. Paoli, "Internal model based fault tolerant control of a robot manipulator," 43rd IEEE Conference on Decision and Control, Atlantis, Paradise Island, Bahamas, December 2004.

[80] Q. Song, W.J. Hu, L. Yin, Y.C. Soh, "Robust Adaptive Dead Zone Technology for Fault-Tolerant Control of Robot Manipulators Using Neural Networks," Journal of Intelligent and Robotic Systems, Vol. 3, pp. 113-137, 2002.

[81] G. Tao, "Adaptive Control Design and Analysis," John Wiley \& Sons, New York, USA, 2003.

[82] A.G. Siqueira, M.H. Terra, M. Bergerman, "Robust Control of Robots Fault Tolerant Approaches," Springer, London, 2011.

[83] G. Tao, S. Chen, X. Tang, S.M. Joshi, "Adaptive Control of Systems with Actuator Failures," Springer, London, 2004.

[84] H.K. Khalil, "Nonlinear System Third Edition," Printice Hall, New Jersey, USA, 2002. 
[85] A.C. Huang, M.C. Chien, "Adaptive Control of Robot Manipulators A Unified Regressor-Free Approach," World Scientific, USA, 2010.

[86] B.C. Kuo, "Digital Control Systems Second Edition," Oxford University Press, New York, 1992.

[87] C.T. Chen, "Linear System Theory and Design," Oxford University Press, New York, 1999.

[88] S. Cetinkunt, "Mechatronics," John Wiley \& Sons, New York, USA, 2007.

[89] G.F. Franklin, J.D. Powell, A.E. Naeini, "Feedback Control of Dynamic System," Pearson Higher Education, New Jersey, USA, 2010.

[90] G. Liu and Z. Li, "A Unified Geometric Approach to Modeling and Control of Constrained Mechanical Systems" IEEE transaction on Robotics and Automation, vol.18, no. 4, pp.574-587, 2002.

[91] A. Cristofaro, M. M. Polycarpou and T. A. Johansen, "Fault diagnosis and faulttolerant control allocation for a class of nonlinear systems with redundant inputs," 54th IEEE Conference on Decision and Control, pp. 5117-5123, 2015.

[92] G. Aly, A. Badr, and Z. Binder, "Multi-model control of MIMO systems: location and control algorithms," International journal of systems science, vol. 19, no. 9, pp. 1687-1698, 1988.

[93] A. Badr, Z. Binder, R. PERRET, et al, "A multi-model tracking controller for distributed control system," A bridge between control science and technology, vol. 5, pp. 2823-2828, 1985. 
[94] M. Ciliz, K. and K. S. Narendra, "Adaptive control of robotic manipulators using multiple models and switching," The International journal of robotics research, vol. 15, no. 6, pp. 592-610, 1996.

[95] M. K. Ciliz and K. S. Narendra, "Intelligent control of robotic manipulators: a multiple model based approach," iros. IEEE, pp. 2422, 1995.

[96] M. K. Ciliz and K. S. Narendra, "Multiple model based adaptive control of robotic manipulators," Proceedings of the 33th IEEE Conference on Decision and Control, vol. 2, pp. 1305-1310, 1994.

[97] M. Fu, and B. R. Barmish, "Adaptive stabilization of linear systems via switching control," IEEE Transactions on Automatic Control, vol. 31, no. 12, pp. 1097-1103, 1986.

[98] R. Kelly, R. Carelli, M. Amestegui and R. Ortega, "An adaptive impedance control of robot manipulator," IEEE Conference on Robotics and Automation, pp. 572-577, 1987.

[99] A. U. Levin, and K. S. Narendra, "Control of nonlinear dynamical systems using neural networks: Controllability and stabilization," IEEE Transactions on Neural Networks, vol. 4, no. 2, pp. 192-206, 1993.

[100] A. U. Levin, and K. S. Narendra. "Control of nonlinear dynamical systems using neural networks. II. Observability, identification, and control," IEEE Transactions on Neural Networks, vol. 7, no. 1, pp. 30-42, 1995.

[101] W. Li, J. J. E. Slotine, "An indirect adaptive robot controller," Systems and control letters, vol. 12, no. 3, pp. 259-266, 1989, 
[102] R.H. Middleton and G.C. Goodwin, "Adaptive computed torque control for rigid link manipulators," IEEE Conference on decision and control, pp. 68-73, 1986.

[103] R. H. Middleton, G. C. Goodwin, D. J. Hill, and D. Q. Mayne, "Design issues in adaptive control," IEEE Transactions on Automatic Control, vol. 33, no. 1, pp. $50-58,1988$.

[104] K. S. Narendra and C. Xiang, "Adaptive control of discrete-time systems using multiple models," Proceedings of the 37th IEEE Conference on Decision and Control, vol. 4, pp. 3978-3983, 1998.

[105] K. S. Narendra and C. Xiang, "Adaptive control of discrete-time systems using multiple models," IEEE Transactions on Automatic Control, vol. 45, no. 9, pp. 1669-1686, 2000.

[106] K, S. Narendra and J. Balakrishnan, "Improving transient response of adaptive control systems using multiple models and switching," IEEE Transactions on Automatic Control, vol. 39, no. 9 pp. 1861-1866, 1994.

[107] K. S. Narendra and J. Balakrishnan, "Intelligent control using fixed and adaptive models," Proceedings of IEEE 33rd Conference Decision and Control, 1 vol. 2, pp. 1680-1685, 1994.

[108] K. S. Narendra, J. Balakrishnan, and K. M.Ciliz, "Adaptation and learning using multiple models, switching, and tuning," IEEE Control Systems, vol. 15, no. 3, pp. 37-51, 1995.

[109] K. S. Narendra and K. Parthasarathy, "Identification and control of dynamical systems using neural networks," IEEE Transactions on Neural Networks, vol. 1, no. 1, pp. 4-27, 1990. 
[110] R. Ortega, and M.W. Spong, "Adaptive motion control of rigid robots: a tutorial," Automatica, vol. 25, no. 6, pp. 877-888, 1989.

[111] J. J. E. Slotine and W. Li, "On the adaptive control of robot manipulators," International Journal of Robotics Research, vol. 6, no. 3, pp. 49-59, 1987.

[112] J. J. E. Slotine and W. Li, "Adaptive strategies in constrained manipulation," IEEE International Conference on Robotics and Automation, pp. 595-601, 1987.

[113] L. L. Whitcomb, A. Rizzi, and D. E. Koditschek, "Comparative Experiments with a New Adaptive Controller for Robot Arms," IEEE Transactions on Robotics and Automation, vol. 9, no.1, pp. 59-70, 1993. 